\title{
Genderstudies
}

Carmen Leicht-Scholten (Hg.)

\section{„) Gender and Science}

Perspektiven in

den Natur- und

Ingenieurwissenschaften 
Carmen Leicht-Scholten (Hg.)

»Gender and Science« 

Carmen Leicht-Scholten (Hg.)

"Gender and Science"

Perspektiven in den Natur- und Ingenieurwissenschaften 
Bibliografische Information der Deutschen Bibliothek

Die Deutsche Bibliothek verzeichnet diese Publikation in der Deutschen Nationalbibliografie; detaillierte bibliografische Daten sind im Internet über http://dnb.ddb.de abrufbar.

(C) 2007 transcript Verlag, Bielefeld

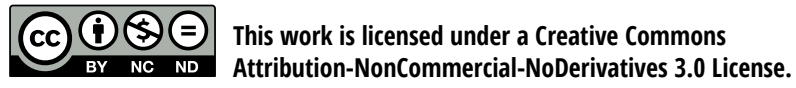

Umschlaggestaltung: Kordula Röckenhaus, Bielefeld Lektorat \& Satz: Carmen Leicht-Scholten

Druck: Majuskel Medienproduktion GmbH, Wetzlar ISBN 978-3-89942-674-8

Gedruckt auf alterungsbeständigem Papier mit chlorfrei gebleichtem Zellstoff.

Besuchen Sie uns im Internet: http://www.transcript-verlag.de

Bitte fordern Sie unser Gesamtverzeichnis und andere Broschüren an unter:info@transcript-verlag.de 


\section{Inhalt}

Vorwort

Gender Mainstreaming and Gender Studies Ein Spannungsfeld

Gender Mainstreaming - Mehr als nur ein Papiertiger? -

Wie viel Gender ist im Mainstream der Wissenschaften?

CARMEN LEICHT-SCHOLTEN

HENRIKE WOLF

Gender im Mainstream der Hochschulentwicklung.

Institutionalisierung von Frauenförderung und Gender

Mainstreaming am Beispiel MEDUSE

RENATE KLEES-MÖLLER

BÄRBEL ROMPELTIEN

Das Studienfach Gender Studies: ein Erfahrungsbericht über die Einrichtung des interdisziplinären Faches

im Bundesland Bayern

CORINNA ONNEN-ISEMANN 
Gender und Innovationen - Erfahrungen aus dem Projekt „Discover Gender!“

NINA BESSING

HELGA LUKOSCHAT

A Fascinating History of Curious Careers: Women in Science and Engineering in the Netherlands, 1650-2005

MINEKE BOSCH

Gender \& Science - Vielfältige Perspektiven

Nachhaltige Ingenieurausbildung

CHRISTINE WÄCHTER

Ten Keys to Invole More Women in Academic Computing 119

ALLAN FISHER

JANE MARGOLIS

Informatik und Geschlechtergerechtigkeit in Deutschland Annäherungen

BRITTA SCHINZEL

Geschlechtergerechtes Lehren und Lernen in Naturwissenschaft und Technik. Aktiv, kooperativ und authentisch durch Kontextorientierung und reflexive Koedukation

MONIKA BESSENRODT-WEBERPALS

Gender und Diversity in Ingenieurwissenschaften 157

SUSANNE IHSEN

Frauengesundheit in der medizinischen Versorgung 171

CLAUDIA HORNBERG

MiCHAELA WEISHOFF-HOUBEN

Die Autorinnen und Autoren 183 


\section{Vorwort}

Die Beiträge dieses Bandes gehen auf eine interdisziplinäre Ringveranstaltung zurück, die im Sommersemester 2006 an der RheinischWestfälischen Technischen Hochschule (RWTH) in Aachen im Rahmen eines anwendungsorientierten Forschungsprojektes stattfand.

Ausgangspunkt des am Institut für Soziologie angesiedelten Projektes war es zu einem hochschulweiten Dialog zum Thema Gender and Science anzuregen und Möglichkeiten aufzuzeigen, wie Gender-Aspekte in Forschung und Lehre an einer technischen Hochschule integriert werden und dadurch Forschungsperspektiven erweitert werden können.

Das Kooperationsprojekt zwischen dem Instituts für Soziologie und dem Frauenprojekt der RWTH Aachen (Zusammenschluss von Studentinnen der RWTH und der FH) unter Leitung der Sozialwissenschaftlerin Dr. Carmen Leicht-Scholten wurde von der Gleichstellungsbeauftragten der RWTH und dem freien Zusammenschluss der Studentinnenschaft unterstützt. Die Schirmherrschaft für das Projekt übernahm der Rektor der Hochschule Prof. Dr. Burckhard Rauhut.

Gefördert wurde das Projekt durch das Ministerium für Innovation, Wissenschaft, Forschung und Technologie des Lands NRW, ohne die eine Realisierung gar nicht möglich gewesen wäre.

An dieser Stelle möchte ich mich ganz herzlich bei allen Personen bedanken, die bei dem Projekt mitgearbeitet haben.

Bei den befragten Professorinnen und Professoren der RWTH für ihre Mitarbeit und ihr Engagement. Des Weiteren bei Lea Heuser und Steffi Houben, die als Vertreterin des Frauenprojektes das gemeinsame Projekt initiiert und als studentische Mitarbeiterinnen im Projekt mitgearbeitet haben.

Bei Dirk Kelzenberg, der maßgeblich für die Auswertung der Befragung verantwortlich war. Nicht zuletzt bei meinen studentischen Mitar- 
beiterinnen Miriam Lorenz, die für die Erstellung des Manuskriptes verantwortlich war und Petra Kehr, die an unterschiedlichsten Stellen im Projekt mitarbeitete.

Dr. Carmen Leicht-Scholten, Aachen im Mai 2007 


\section{Einleitung}

Um einen Dialog zum Thema Gender and Science an einer technischen Hochschule anzuregen, ging das stark anwendungsorientierte Forschungsvorhaben in zwei Schritten vor. Im ersten Schritt stand der Versuch einer Standortbestimmung, d.h. eine Annäherung an die Klärung der Frage, welche Bedeutung genderbezogenen Fragestellungen in Wissenschaft und Forschung von Seiten der aktiven Professorinnen und Professoren an der Hochschule eingeräumt werden, um dann in einem nächsten Schritt anhand der Beiträge internationaler Expertinnen und Experten Möglichkeiten aufzuzeigen, wie Gender-Aspekte in Forschung und Lehre integriert werden können und best practice Modelle vorzustellen.

Die Forschung zu Fragen der Geschlechterverhältnisse hat sich seit ihren Anfängen vor ca. 30 Jahren stark verändert. Hervorgegangen aus der Frauenforschung beschäftigen sich die Gender Studies mit der Bedeutung der sozialen Kategorie Geschlecht (Gender) und der Bedeutung von Geschlechterverhältnissen in ihrem sozialen Zusammenhang. So hat sich das Spektrum der beteiligten Fachdisziplinen erweitert, deren Bandbreite inzwischen von den Sozialwissenschaften, über Physik oder Mathematik bis hin zur Medizin reicht, während die Anfänge der Frauenforschung fast ausschließlich in den Sozialwissenschaften lagen. Die Forschungsfragen sind ebenso wie die theoretischen Zugänge vielfältiger geworden.

Diese Ausdifferenzierung lässt sich auch in der Forschung zum Thema Wissenschaft und Geschlecht feststellen. Hier hat sich insbesondere der Fokus der Fragestellungen verschoben. In den frühen Forschungen - und damit einhergehend den daraus entwickelten Entscheidungen für die Gleichstellungspolitik - lag der Forschungsschwerpunkt von Seiten der Organisationen auf struktureller Ebene, zum anderen aber 
bei den Frauen selbst, was sich auch in der Bezeichnung Frauenforschung und Frauenpolitik/Gleichstellungspolitik zeigt.

Die Studien zur Unterrepräsentanz von Frauen in der Wissenschaft beschäftigten sich primär mit der Analyse konkreter Situationen von Frauen in der Wissenschaft und weisen insbesondere auf die schlechtere Positionierung und Dotierung der Wissenschaftlerinnen trotz formal gleicher Qualifikation hin (vgl. Nowotny 1986; Ursula Bock u.a.1983).

Seit den 90er Jahren findet auch vermehrt eine Auseinandersetzung mit Frauen in den Naturwissenschaften statt, die auch im Zusammenhang mit der aktuellen Diskussion um den geringen Anteil von Frauen in technischen und naturwissenschaftlichen Fächern steht, wobei neben der Frage nach der Beteiligung von Frauen in den Disziplinen auch eine Vielfalt der Themen und methodischen Zugänge konstatiert werden kann. Inhaltlich beziehen sich die Arbeiten auf individuelle bzw. biografische Aspekte der Frauen (Wolffram 2003), Analyse diskriminierender struktureller Bedingungen, geschlechtsspezifischer Differenzen im Karriereverhalten (Allmendinger 1999) sowie auf gesellschaftlich bedingte Barrieren hinsichtlich der Vereinbarkeitsthematik und bestimmter Rollenzuschreibungen an Frauen sowie fächerspezifischen Untersuchungen (Götschel 2001; Wobbe 2003; Heintz 2004).

Gerade in den letzten Jahren lässt sich eine Ausweitung der Perspektive auch auf Aspekte der beteiligten Organisationen der Wissenschaft feststellen (Färber 2002), wobei sich als aktueller Trend eine Verknüpfung von individuellen (Berufs-)Verläufen und Organisationsstrukturen ausmachen lässt. Im Anschluss an die Theorie Bourdieus gibt es diverse Studien, die sich mit der Frage beschäftigen, wie Wissenschaft als soziales Feld funktioniert und wie Frauen sich in diesem Spiel positionieren können (Krais 2000; Zimmermann 2000; Engler 2001; Beaufays 2003).

Eine ,wechselseitige Konstitution von Geschlecht und Wissenschaft" führt dazu, in den unterschiedlichen wissenschaftlichen Disziplinen auch spezifische Bedingungen und Faktoren identifizieren zu lassen, die geschlechterdifferente Bedingungen und Verhaltensweisen erzeugen und damit die Geschlechtszugehörigkeit sozial relevant werden lassen (vgl. Heintz 2004).

Hinzu kommt auch die Kritik an der Auswahl der Forschungsthemen und der Interpretation der Ergebnisse bis hin zu einer grundsätzlichen Methodenkritik („Gender in Science“, Harding 1994). Die Prioritäten, die gesetzt und die Forschungsfragen, die gestellt werden, die Auswahl von Versuchspersonen oder von Untersuchungsobjekten, die Interpretation von Ergebnissen und die Sprache, die dabei verwendet wird - das 
alles kann nicht unabhängig von Gender - und weiteren Kategorien betrachtet werden (vgl. Wajcman 2004; Ebeling 2006).

Durch diese neuen Forschungsansätze werden wieder neue Fragen aufgeworfen. So ist zu klären, an welcher Stelle in den verschiedenen Fächern Gender-Kategorien auf welchen unterschiedlichen Ebenen ins Spiel kommen, wo diese bedeutsam werden, bzw. nicht bedeutsam werden. Demzufolge müssen allgemeine Betrachtungen über die Einbeziehung von Gender-Aspekten ergänzt werden, durch Gender-Forschung, die die einzelnen Disziplinen in den Blick nimmt.

Während die Gender Studies auf der wissenschaftlichen Ebene die Kategorie Geschlecht als soziale Strukturkategorie zu durchschauen und im nächsten Schritt auch zu verändern suchen, zielt die Strategie des Gender Mainstreaming direkt auf die Institutionalisierung von Geschlechtergerechtigkeit.

Wenn sich Gender Mainstreaming nicht nur auf Mittel und Strategien bezieht, sondern Reorganisation einer Institution auf allen hierarchischen Ebenen im Hinblick auf Geschlechtergerechtigkeit anstrebt und die Geschlechterfrage als wesentliches Kriterium für den Output betrachtet, - d.h. im Felde der Wissenschaft für die Entwicklung von Forschungsfragen und Forschungsprojekten - dann kann das Konzept des Gender Mainstreaming in Verbindung mit den ,,verunsicherungstheoretischen“ (vgl. Degele 2000: 13) Grundannahmen der Gender Studies ein radikaler Reorganisationsansatz werden.

Gender Mainstreaming als eine politische Strategie betrifft damit Wissenschaft nicht nur im Hinblick auf die Präsenz von Frauen an der Institution Hochschule. Ebenso geht es um das Durchdringen der Forschungs- und Lehrinhalte mit der Kategorie Gender. Dies betrifft zum einen die Implementierung eines Faches wie Gender Studies, zum anderen aber - und das ist langfristig viel wichtiger - das Mainstreaming der ganz normalen Fächer.

Mit der Einführung der Kategorie Gender werden wissenschaftskritische Fragen an das jeweilige Fach und an die Hierarchie der Fächer gestellt und Kategorien, nach denen Wissen jeweils geordnet und strukturiert wird, hinterfragt. Durch die Einbeziehung von Gender als Analysekategorie können Sichtweisen erweitert, und damit ein wesentlicher Beitrag zu einem interdisziplinären Wissenschaftsverständnis geleistet werden. 
Der Band gliedert sich in Anlehnung an die Gender Studies und das Spannungsfeld zwischen Gender Studies und Gender Mainstreaming in zwei Teile.

Die Beiträge des ersten Teils liegen innerhalb des Spannungsfeldes zwischen Gender Studies und Gender Mainstreaming.

Der erste einleitende Beitrag von Carmen Leicht-Scholten und Henrike Wolf geht anhand einer Befragung von Professorinnen und Professoren der Frage nach, inwieweit das im Leitbild der technischen Hochschule formulierte Konzept des Gender Mainstreaming von den Lehrenden auch im wissenschaftlichen Alltag umgesetzt wird und ob es Eingang in Forschung und Lehre der Hochschule findet. Die Ergebnisse der Befragung machen deutlich, dass Gender noch nicht im Mainstream der Wissenschaften angekommen ist.

Ein konkretes Beispiel für die institutionelle Umsetzung von Gender Mainstreaming und Frauenförderung im Hochschulmanagement geben Renate Klees-Möller und Bärbel Rompeltien. Am Beispiel Meduse, eines Geschäftsbereichs des Zentrums für Hochschul- und Qualitätsentwicklung zeigen sie eine mögliche Perspektive auf, wie eine erfolgreiche Gender-Strategie integriert werden kann.

Die Chancen und Grenzen eines interdisziplinären Studienfaches Gender Studies beschreibt Corinna Onnen-Isemann in Ihrem Erfahrungsbericht über die Einrichtung des interdisziplinären Faches im Bundesland Bayern. Die Chance Gender-Aspekte im Rahmen eines interdisziplinär angelegten Studiengangs Gender Studies in die Curricula einer Hochschule einzubeziehen ist innerhalb der unterschiedlichen Disziplinen nur dann möglich, wenn das Fach selbst institutionell und strukturell stark eingebunden ist.

Die Bedeutung der Gender- und Innovationsforschung für die anwendungsorientierte Forschung und Technikentwicklung beschreiben Nina Bessing und Helga Lukoschat im Rahmen der Erfahrungen des von 2003-2006 von der Fraunhofer Gesellschaft durchgeführten Projektes „Discover Gender“. Das von einem interdisziplinären Team durchgeführte Projekt zeigt anschaulich, welche Bedeutung die Berücksichtigung von Gender-Aspekten für eine anwendungsorientierte Forschung hat und welches Innovationspotential darin steckt.

Mineke Bosch zeichnet in ihrem Beitrag die lange Tradition von erfolgreichen Wissenschaftlerinnen in den Natur- und Ingenieurwissenschaften nach. Seit dem 17. Jahrhundert gab es immer wieder Wissenschaftlerinnen wie Maria Sybilly Merin, Maria Winkelmann u.a., die die Wissenschaften entscheidend vorangetrieben haben. Das den Frauen in der Neuzeit zugeschriebene fehlende Technikinteresse führt sie auf Er- 
ziehungsreformen im 19. Jahrhundert zurück, durch die Frauen mehr und mehr ausgegrenzt wurden.

In den Beiträgen des zweiten Teils wird der Fokus von Gender und Wissenschaft auf einzelne Wissenschaftsdisziplinen gerichtet.

Christine Wächter zeigt anhand der Ergebnisse des internationalen Forschungsprojektes „WomEng - Creating Cultures of Success for Women Engineers“ auf, dass erfolgreiche Ingenieurinnenkarrieren in hohem Maße von institutionellen und organisatorischen Rahmenbedingungen in Ausbildungseinrichtungen und Betrieben abhängen. Die mittels einer Fragebogenerhebung durchgeführte Studie untersuchte die Einflussfaktoren auf Studien- bzw. Berufsverläufe von Ingenieurinnen in sieben europäischen Ländern. Dass nicht nur der Erfolg, sondern auch die Beteiligung von Frauen in natur- und ingenieurwissenschaftlichen Disziplinen von institutionellen und organisatorischen Rahmenbedingungen entscheidend beeinflusst wird, zeigt auch der Beitrag von Allan Fisher und Jane Margolis. Mit einer Veränderung der Curricula im Fachbereich Informatik konnte der Frauenanteil in der Informatik an der Carnegie Mellon Universität von 9 auf 42 \% erhöht werden. Darauf aufbauend fasst der Beitrag die zehn Leitideen zusammen, die maßgeblich für die Erhöhung des Frauenanteils in der Informatik verantwortlich sind.

Mit dem Frauenanteil in der Informatik befasst sich auch der Beitrag von Britta Schinzel. Nach einem Kulturvergleich unter Berücksichtigung der Frauenbeteiligung im Informatik-Studium, macht sie konkrete Vorschläge für eine Veränderung dieses Studiengangs in Deutschland, um mehr Frauen für dieses Studienfach zu motivieren und auch im Studium zu halten.

Monika Bessenrodt-Weberpals macht in ihrem Beitrag deutlich, dass eine gendergerechte Sichtweise auch in der Fachdidaktik eingesetzt werden muss, um den Studien-Erfolg von Studentinnen und Studenten nachhaltig zu verbessern.

„Diversity“ ist das Thema des Beitrags von Susanne Ihsen. Die betriebliche Berücksichtigung verschiedener Personengruppen wird in internationalen Unternehmen nutzbar gemacht, um den Ansprüchen von Kundinnen und Kunden sowie Märkten in unterschiedlichen Kulturen und Regionen gerecht zu werden. Gemeinsam mit der Erkenntnis in Unternehmen, dass künftig nicht nur Fachfrauen fehlen, sondern dass insgesamt zu wenige Ingenieurinnen und Ingenieure zur Verfügung stehen, führt dazu, eine Vielzahl von Programmen und Maßnahmen aufzulegen. Claudia Hornberg und Michaela Weishoff-Houben zeigen in ihrem Vortrag die Bandbreite der geschlechterspezifischen Forschung auf, die sich 
in den letzten Jahren etabliert hat. Es werden sowohl die biomedizinischen Risiken, die Unterschiede im Krankheitsspektrum von Frauen und Männern, aber auch Unterschiede zwischen Männern und Frauen in Bezug auf verhaltensbedingte Risiken, die Inanspruchnahme medizinischer Leistungen und die Medikamentenverordnung untersucht.

Die Beiträge der genannten Expertinnen und Experten haben wesentlich zum Erfolg der Ringveranstaltung beigetragen, die mit einer durchschnittlichen Teilnehmerinnenzahl von über 100 Studentinnen und Studenten aus den unterschiedlichsten Disziplinen sehr gut besucht war (vgl. Leicht-Scholten 2006). Da auch einige Professorinnen und Professoren an den Veranstaltungen teilnahmen, lässt sich zusammenfassend sagen, dass es durchaus gelungen ist einen Dialog zum Thema Gender and Science anzuregen. Ein Dialog, der sowohl die Perspektiven der einzelnen wissenschaftlichen Disziplinen als auch der Hochschule als Institution erweitern kann.

\section{Literatur}

Allmendinger, Jutta/Brückner, Hannah/Fuchs, Stefan/Stebut von, Janina (1999): Eine Liga für sich? Berufliche Werdegänge in der Max Planck Gesellschaft. In: Vielfältige Verschiedenheiten. Geschlechterverhältnisse in Hochschule, Studium und Beruf, hg.v. Ayala Neusel/Angelika Wetterer, Frankfurt/New, S. 193-220.

Beaufays, Sandra (2003): Wie werden Wissenschaftler gemacht? Beobachtungen zur wechselseitigen Konstitution von Geschlecht und Wissenschaft.

Bock, Ursula/Braszeit, Anne/Schmerl, Christiane (Hg.) (1983): Frauen an Universitäten: Zur Situation von Studentinnen und Hochschullehrerinnen in der männlichen Wissenschaftshirarchie, Frankfurt a.M./ New York.

Degele, Nina (2000): Anpassen oder unterminieren: Zum Verhältnis von Gender Mainstreaming und Gender Studies. In: Freiburger FrauenStudien, S. 1-13.

Engler, Stefanie (2001): „In Einsamkeit und Freiheit?“ Zur Konstruktion der wissenschaftlichen Persönlichkeit auf dem Weg zur Professur, Konstanz.

Ebeling, Smilla/Schmitz, Sigrid (2006): Geschlechterforschung und Naturwissenschaften - Einführung in ein komplexes Wechselspiel, Wiesbaden. 
Färber, Christine (2002): Frauen auf die Lehrstühle durch Gender Mainstreaming? Eine neues gleichstellungspolitisches Konzept und seine Bedeutung für den Hochschulbereich. In : Gender Mainstreaming -eine Innovation in der Gleichstellungspolitik. Zwischenberichte aus der politischen Praxis, hg.v. Silke Bothfeld/Sigrid Gronbach/ Barbara Riedmüller, Frankfurt a.M./New York, S. 107-131.

Götschel, Helene/Daduna, Hans (Hg.)(2001): Frauen und Geschlechterforschung zu Mathematik und Naturwissenschaften, Mössingen.

Harding, Sandra (1994): Das Geschlecht des Wissens. Frauen denken die Wissenschaft neu, Frankfurt a.M./New York.

Heintz, Bettina/Merz, Martina/Schumacher, Christina (2004): Wissenschaft die Grenzen schafft. Geschlechterkonstellationen im disziplinären Vergleich, Bielefeld.

Krais, Beate (2000): Wissenschaftskultur und Geschlechterforschung. Über die verborgenen Mechanismen männlicher Dominanz in der akademischen Welt, Frankfurt.

Leicht-Scholten, Carmen (2006): Gender and Science - Perspektiven für die Wissenschaft. In: ADA-MENTORING, 16. Ausgabe, Dortmund, S. 13-16.

Neusel, Ayala/Angelika Wetterer (Hg.) (1999): Vielfältige Verschiedenheiten. Geschlechterverhältnisse in Hochschule, Studium und Beruf, Frankfurt a.M./New York.

Nowotny, Helga (1986): Über die Schwierigkeiten des Umgangs von Frauen mit der Institution Wissenschaft. In: Wie männlich ist die Wissenschaft, hg.v. Helga Nowotny et.al., Frankfurt a.M., S. 17-30.

Wajcman, Judy (2004): TechnoFeminism, Cambridge.

Wobbe, Theresa (2003): Zwischen Vorderbühne und Hinterbühne. Beiträge zum Wandel der Geschlechterbeziehungen in der Wissenschaft vom 17. Jahrhundert bis zur Gegenwart, Bielefeld.

Wolffram, Andrea (2003): Frauen im Technikstudium. Belastungen und Bewältigung in sozialen Studiensituationen, Münster.

Zimmermann, Karin (2000): Spiele mit der Macht in der Wissenschaft. Passfähigkeit und Geschlecht als Kriterien der Berufung, Berlin. 

Gender Mainstreaming and Gender Studies - Ein Spannungsfeld 



\section{Gender Mainstreaming - Mehr als nur ein Papiertiger? - Wie viel Gender ist im Mainstream der Wissenschaften?}

CARMEN LEICHT-SCHOLTEN UND HENRIKE WOLF

Gender Mainstreaming hat nach Politik und Wirtschaft inzwischen auch Einzug in die Hochschulen gehalten. Der Begriff findet sich in den Leitbildern von Hochschulen ebenso wie in den Zielvereinbarungen. Aber welches Konzept steht dahinter? Anhand einer Befragung von Professorinnen und Professoren an einer technischen Hochschule wird der Frage nachgegangen, inwiefern das im Leitbild der Hochschule formulierte Genderprofil von den Lehrenden auch im wissenschaftlichen Alltag umgesetzt wird und ob es Eingang in Forschung und Lehre der Hochschule findet.

\section{Ausgangslage}

Die Zukunftsfähigkeit der europäischen Hochschulen wird in den kommenden Jahren verstärkt durch zwei zentrale Fragestellungen bestimmt. Zum einen verschärft die demografische Entwicklung den Fach- und Führungskräfte-Mangel gerade auch an den Hochschulen, womit die Rekrutierung des wissenschaftlichen Personals ein wichtiger Qualitätsfaktor für den Standort Hochschule sein wird. Zum anderen wird die Wettbewerbsfähigkeit der europäischen Hochschulen im internationalen Vergleich von einer Qualitätssicherung in der Lehre, der Förderung der Innovationsfähigkeit in der Forschung und der Internationalisierung der Hochschulen in Forschung und Lehre abhängen. Zielsetzungen, die nur dann zu erreichen sind, wenn aus dem gesamten wissenschaftlichen Po- 
tential geschöpft wird. Die Einbeziehung von Chancengleichheit in die Forschungspolitik ist damit nicht mehr nur eine Frage der Gerechtigkeit, sondern auch eine Frage des wissenschaftlichen Leistungsniveaus und der Effizienz (European Commission 2004).

Diese „Ökonomisierung“ der Gleichstellungspolitik (vgl. dazu Bereswill 2004) findet ihren Ausdruck im Konzept des Gender Mainstreaming. Verstärkt durch die Politik der Europäischen Union und die zunehmende Koppelung der Vergabe von Forschungsgeldern an die Einbeziehung von Gender-Aspekten bei der Umsetzung und Durchführung von Forschungsvorhaben hält das Konzept des Gender Mainstreaming auch in die Hochschulen Einzug.

Die Rheinisch-Westfälische Technische Hochschule Aachen zeichnet sich durch starke Natur- und Ingenieurwissenschaften aus, was sich u.a. daran zeigt, dass von den fast 140 Millionen eingeworbenen Drittmitteln im Jahre 2005 über $70 \%$ von den Ingenieurwissenschaften, und mehr als $15 \%$ von den Naturwissenschaften (vgl. Zahlenspiegel 2005: 74) eingeworben wurden. ${ }^{1}$ Ganz im Gegensatz zu dieser Spitzenposition in Deutschland steht die Beteiligung von Frauen in diesen Disziplinen. Im nationalen Hochschulranking nach Gleichstellungsaspekten teilt sich die Hochschule mit anderen technischen Hochschulen die Schlusspositionen (CEWS 2005).

Welche Bedeutung wird Gender-Aspekten an der RWTH Aachen beigemessen? Handelt es sich ausschließlich um „Antragslyrik“ oder ist das in ihrer Berücksichtigung liegende Innovationspotential schon ein Teil der vielen erfolgreich eingeworbenen Drittmittel?

Was bedeutet es für eine Hochschule ,in alle Entscheidungsprozesse die Perspektive des Geschlechterverhältnisses einzubeziehen und alle Entscheidungsprozesse für die Gleichstellung der Geschlechter nutzbar zu machen"? ${ }^{2}$

1 Die RWTH Aachen nimmt damit in der Bundesrepublik immer einen Spitzenplatz ein, was Drittmitteleinwerbung betrifft.

2 Wie die Expertenkommission der EU Gender Mainstreaming definiert (vgl.: Expertenkommission der Europäischen Union 1999). 


\section{Gender Mainstreaming versus Gender Studies?}

Während Gender Studies auf der wissenschaftlichen Ebene die Kategorie Geschlecht als soziale Strukturkategorie zu durchschauen und im nächsten Schritt auch zu verändern suchen, zielt die Strategie des Gender Mainstreaming direkt auf die Institutionalisierung von Geschlechtergerechtigkeit (vgl. Meuser 2004).

Abb. 1: Gender Mainstreaming versus Gender Studies

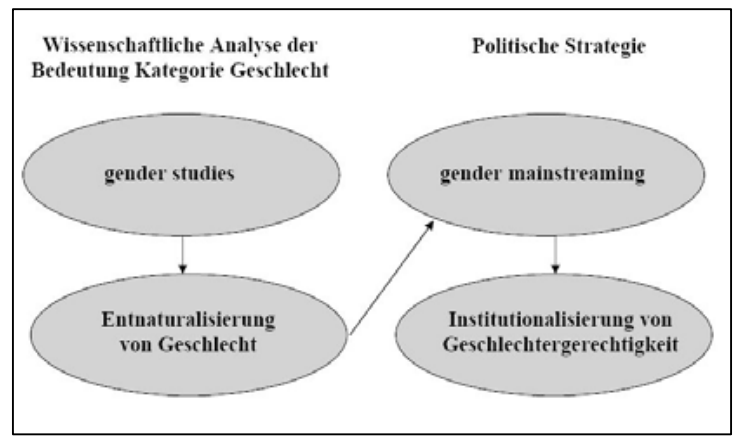

\section{Quelle: Eigene Darstellung}

Dabei lassen sich drei Varianten des Konzeptes unterscheiden: die deskriptive Methode, die normative Strategie und der „radikale Reorganisationsansatz" (vgl. Degele 2000: 2).

Als deskriptive Methode überprüft Gender Mainstreaming sämtliche politische Aktivitäten und Entscheidungsprozesse im Hinblick auf ihre Geschlechterrelevanz - mit analytischer Distanz. Mit Methoden wie geschlechterspezifischer Statistiken etc. Als deskriptive Methode lässt sich Gender Mainstreaming auch als Absichtserklärung instrumentalisieren, um Anerkennung, Fördermittel und Prestige zu gewinnen, letztlich aber alles zu belassen, wie es ist. Ohne normativen Bezugsrahmen bleibt Gender Mainstreaming damit völlig frei verfüg- und instrumentalisierbar für die unterschiedlichsten politischen Zielsetzungen.

Einen Schritt weiter geht Gender Mainstreaming als normative politische Strategie, wie sie auch die Expertenkommission der Europäischen Union formuliert hat, indem die oben skizzierten Analysen unter der Fragestellung durchgeführt werden, welchen Beitrag sie zur Chancengleichheit leisten und wie eine solche auch tatsächlich zu erreichen ist. Wenn sich aber Gender Mainstreaming nicht nur auf Mittel und Strategien bezieht, sondern wirklich die gesamte Organisation auf allen hie- 
rarchischen Ebenen im Hinblick auf Geschlechtergerechtigkeit reorganisiert und die Geschlechterfrage als wesentliches Kriterium für den Output betrachtet wird, d.h. im Felde der Wissenschaft für die Entwicklung von Forschungsfragen und Forschungsprojekten, dann kann das Konzept des Gender Mainstreaming in Verbindung mit den „verunsicherungstheoretischen“ (vgl. Degele 2000: 13) Grundannahmen der Gender Studies ein radikaler Reorganisationsansatz werden.

Abb. 2: Die drei Varianten des Konzeptes Gender Mainstreaming

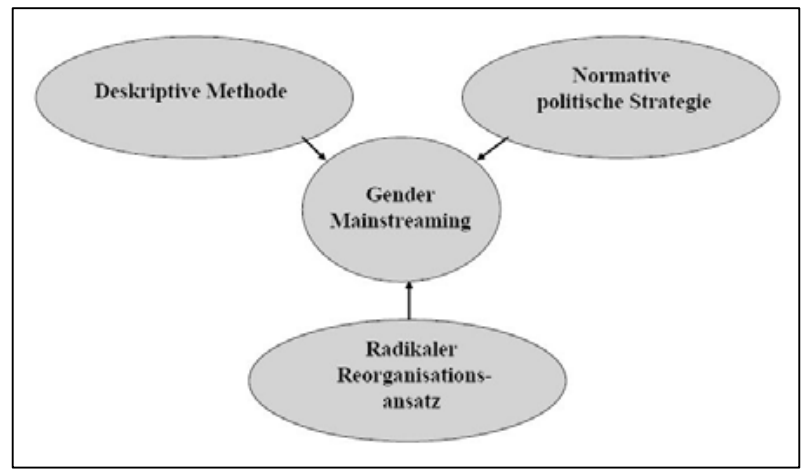

Quelle: Eigene Darstellung

Gender Mainstreaming als eine politische Strategie betrifft damit Wissenschaft nicht nur im Hinblick auf die Präsenz von Frauen an der Institution Hochschule. Ebenso geht es um das Durchdringen der Forschungs- und Lehrinhalte mit dem Thema Gender. Dies betrifft zum einen die Implementierung eines Faches wie Gender Studies, zum anderen aber - und das ist langfristig viel wichtiger - das Mainstreaming der ganz normalen Fächer. Die Hoffnung ist, dass sich gender studies als eigenes Fach eines Tages überflüssig machen werden, weil sie in die gesamte Wissenschaft diffundiert sind. Gender Mainstreaming der Wissenschaft bedeutet dann, Gender als Kategorie in allen Disziplinen zu verankern - ob als eigenes Thema oder anwendungs- oder projektbezogen.

\section{Das Projekt "Gender and Science“}

Diese Fragen waren der Ausgangspunkt eines anwendungsorientierten Forschungsprojektes am Institut für Soziologie der RWTH Aachen (siehe dazu auch www.genderandscience.de). 
Ziel des Projektes war es einen breiten, hochschulweiten Dialog anzuregen und Möglichkeiten aufzuzeigen, wie Gender-Aspekte in Forschung und Lehre einer technischen Hochschule integriert werden und dadurch Forschungsperspektiven erweitert werden können. Im Rahmen des Projektes wurde Ende 2005 eine Befragung aller Professorinnen und Professoren zum Thema „Gender and Science“ durchgeführt. Parallel zur Befragung wurde eine Dokumentenanalyse von Leitbild und Zielvereinbarungen der Hochschule zum Thema Wissenschaft und Geschlecht durchgeführt. Und schließlich fand im Sommersemester 2006 eine interdisziplinäre Ringvorlesung ${ }^{3}$ statt, deren Beiträge in diesem Band zu finden sind.

\section{Gender Mainstreaming in Leitbild und Zielvereinbarungen der Hochschule}

Die RWTH Aachen bezieht sich sowohl in ihrem Leitbild als auch in den Zielvereinbarungen mit dem Land Nordrhein Westfalen auf das Konzept des Gender Mainstreaming.

So formuliert die Hochschule in ihrem Leitbild: „Die RWTH verfolgt das Ziel, die unterschiedlichen Lebenssituationen und Interessen von Frauen und Männern in allen Bereichen, einschließlich Forschung und Lehre, von vornherein und regelmäßig zu berücksichtigen."(Leitbild der RWTH).

Mit der Berücksichtigung der unterschiedlichen Lebenssituationen von Männern und Frauen auch in Forschung und Lehre geht die Hochschule über eine Strategie der Frauenförderung hinaus. Sie folgt vielmehr dem Konzept des Gender Mainstreaming und trägt der Tatsache Rechnung, dass es eine Wechselbeziehung von Gender und Wissenschaft gibt, die weit mehr umfasst als die Frage nach der numerischen Beteiligung von Frauen in der Wissenschaft. Dieses umfassende Konzept des Gender Mainstreaming findet sich allerdings in den Zielvereinbarungen mit dem Land Nordrhein-Westfalen nicht in dieser Klarheit. Darin heißt es: ,die Chancengleichheit in allen Bereichen ist die Grundlage für die in dieser Vereinbarung beschriebenen Ziele. Sie [die RWTH] setzt sich das Ziel den Anteil der Frauen an den Professuren und die Anzahl der Studentinnen vor allem in den Fächern zu erhöhen,

3 Die interdisziplinäre Ringveranstaltung bot einen Einblick in das breite Spektrum der Genderforschung in den unterschiedlichsten wissenschaftlichen Disziplinen (vgl. Website http://www.genderandscience.de). 
in denen noch ein deutlicher Nachholbedarf besteht" (Zielvereinbarungen II 2005: 2).

Darüber hinaus wird in den Zielvereinbarungen in $\S 7$ ein eigenes „Genderprofil“" formuliert.

\section{Abb. 3: Das Genderprofil an der RWTH Aachen}

\section{$\S 7$ Genderprofil}

(1) Zur Bündelung der an der Hochschule bestehenden vielfältigen Aktivitäten verpflichtet sich die RWTH zum Aufbau eines „Female career center Aachen“.

(2) Die RWTH sieht derzeit bezogen auf ihre Schwerpunkte keine konkreten Ansatzpunkte für genderbezogene Professuren. Die Hochschule wird jedoch jede sinnvolle wissenschaftlich fundierte Anregung hinsichtlich genderbezogener Fragestellungen in ihrer Berufungspolitik mit Nachdruck aufgreifen.

(3) Qualifizierung von Frauen wird durch die Hochschule gefördert. In diesem Zusammenhang werden die derzeit laufenden Projekte fortgeführt. Die RWTH ist bemüht die bestehenden Projekte bei Bedarf um weitere zu ergänzen.

(4) Die RWTH bietet Maßnahmen sowie spezielle Informationsmaterialien für Frauen sowie Studienangebote für Studentinnen in Fächern mit geringem Frauenanteil (z.B. „FordStipendium“ für Maschinenbaustudentinnen, Girls Days, Schnupperuniversität, Kontakte zu Ausbildungsberufen, Institutsaktivitäten) an, mit dem Ziel, den Anteil der Studentinnen und Nachwuchswissenschaftlerinnen in diesen Fächern zu erhöhen.

(5) Die Hochschule verfolgt das Ziel einer gendersensiblen Personalentwicklung. Die in diesem Zusammenhang bestehenden Angebote (Uni\&Kind e.V., Ferienbetreuung für Schulkinder sowie die Servicestelle „Eltern-Service-Büro“) und Qualifizierungsmaßnahmen für weibliche Beschäftigte werden fortgeführt. Um die Vereinbarkeit mit Familie und Beruf zu gewährleisten, strebt die Hochschule an, im Kontext der Beschäftigung von Wissenschaftlerinnen und Wissenschaftlern die Karriereplanung von Familien (Double career) in Kooperation mit den Universitäten Bonn und Köln zu erleichtern. - Darüber hinaus wird die Hochschule Gendertraining anbieten.

Quelle: Zielvereinbarungen II vom 21.01.2005, S.11-12 


\section{Die Befragung}

Ausgangspunkt der Befragung war der Versuch einer Standortbestimmung, d.h. eine Analyse, welche Bedeutung genderbezogenen Fragestellungen in Wissenschaft und Forschung von Seiten der aktiven Professorinnen und Professoren an der RWTH zugemessen wird. Angestrebt wurde eine Totalerhebung aller Universitäts-Professorinnen und Professoren an der Hochschule. Die Befragung wurde mittels eines teilstandardisierten Fragebogens in Form einer Online Befragung im Februar 2005 mit Unterstützung der Hochschulleitung durchgeführt. ${ }^{4}$ Insgesamt wurden 395 Fragebögen an alle aktiven Professorinnen $(\mathrm{N}=20)$ und Professoren der Hochschule verschickt. Die Rücklaufquote liegt mit 59 Fragebögen bei $15 \%$, davon waren 52 männlichen und 7 weiblichen Geschlechts und stellt damit auch für schriftliche Befragungen einen recht unterdurchschnittlichen Wert dar.

Die Teilnahme an Befragungen hängt in hohem Maße von der persönlichen „Betroffenheit“ ab, die von der Fragebogenthematik ausgeht. Ein wichtiger Faktor für ein Vorliegen eines solchen Betroffenheitsbezugs sind hinreichende Kenntnisse bzw. Erfahrungen über den Fragegegenstand und damit zusammenhängende Frageinhalte. Dies scheint bei dieser Untersuchung nur bedingt der Fall gewesen zu sein. Auf Grund niedriger Fallzahlen wird die Auswertung rein deskriptiv durchgeführt, da die Zellenbildung für entsprechende quantitative Analysen größtenteils zu gering ist. Insofern ist nur eine bedingte Aussagekraft der Ergebnisse möglich. ${ }^{5}$

Die einzelnen Wissenschaftsbereiche weisen recht unterschiedliche Rücksendequoten auf (vgl. Abb. 4 ).

\section{Abb. 4: Rücksendequoten aus den Wissenschaftsbereichen}

\begin{tabular}{|lr|}
\hline Ingenieurwissenschaften: & 25 von 133 Personen \\
Naturwissenschaften: & 14 von 108 Personen \\
Geistes- und & \\
Wirtschaftswissenschaften: & 16 von 86 Personen \\
Medizin: & 3 von 80 Personen \\
\hline
\end{tabular}

\section{Quelle: Eigene Darstellung}

4 Der Link wurde über ein vom Rektor unterzeichnetes Anschreiben verschickt und war 14 Tage freigeschaltet.

5 Detaillierte Infos können auf der Website des Projektes (www. genderandscience.de) abgerufen werden. 
Abb. 5: Altersstruktur der Professoren und Professorinnen, die den Fragebogen zurückgesandt haben

\begin{tabular}{|ll}
\hline Alterstruktur: & \\
bis 40 Jahre: & $20,7 \%$ \\
41 bis 50 Jahre: & $36,2 \%$ \\
51 bis 60: & $29,3 \%$ \\
ab 61 Jahre: & $13,8 \%$
\end{tabular}

Quelle: Eigene Darstellung

Ausgehend von den Forschungen der Gender Studies (vgl. ausführlich dazu die Einleitung in diesem Band) gliedert sich die Befragung in drei Bereiche:

Im ersten Bereich standen allgemeine Fragen zum Thema Gender und Gender Mainstreaming und Erfahrungen mit Geschlechterverhältnissen in den einzelnen Wissenschaftsbereichen (Women in Science).

Darauf folgen Fragen nach möglichen Konsequenzen für die Wahl und Darstellung der wissenschaftlichen Gegenstände, Methoden und Zielperspektiven (Science of Gender) und schließlich ging es im dritten Teil um grundsätzlichen Fragen nach Objektivität, Wertfreiheit und Geltung naturwissenschaftlichen Wissens (Gender in Science).

Im Folgenden werden die Ergebnisse der Online-Befragung nach den Bereichen Women in Science, Science of Gender und Gender in Science systematisch dargestellt.

\section{Allgemeine Fragen zum Thema Gender und Gender Mainstreaming}

\section{Bekanntheit und Informiertheit über Gender Mainstreaming}

Während mehr als die Hälfte $(51,7 \%)$ mit dem Begriff Gender Mainstreaming nichts anfangen kann bzw. keine Vorstellung davon hat, ist knapp der Hälfte (48,3 \%) die Bedeutung bekannt bzw. hat schon einmal davon gehört. Dabei erfährt der Begriff den höchsten Bekanntheitsgrad bei den Geistes- und Wirtschaftswissenschaften

Obwohl der Begriff wenig bekannt ist, sehen sich Dreiviertel aller Befragten (77,6 \%) über das Gender-Thema ausreichend informiert. Das restliche Drittel der Befragten (22,4\%), die sich über das Gender-Thema ungenügend informiert fühlen, möchten auch mehr Informationen zum Thema. Bevorzugt werden dabei Informationen wie „Allgemeine Infor- 
mationen zu Wissenschaft und Geschlecht“ (19\%), „Informationen über geschlechtergerechte Lehre“ (17,2\%), „Informationen über hochschulinterne Maßnahmen im Hinblick auf Geschlechtergerechtigkeit“ $(13,8 \%)$, „Erfahrungen mit best-practice Modellen in Natur- und Ingenieurwissenschaften an anderen Hochschulen“ (10,3\%) und „Informationen über Projekte für Frauen“ (8,6 \%). Nur die wenigsten wünschen sich „Screenings auf Gender-Aspekte“ (6,9 \%), Gender-Trainings $(6,9 \%)$ und „Studien zu Gender-Differenzierungen in Natur- und Ingenieurwissenschaften“ $(5,2 \%)$.

\section{Wichtigkeit von Gender Mainstreaming an der Hochschule}

Auf die Frage nach der Relevanz von Gender Mainstreaming für die Hochschule machen über $60 \%$ der Befragten keine eindeutige Aussage. Für mehr als ein Viertel der Befragten ist das Thema Gender Mainstreaming an der Hochschule nicht wichtig (insbesondere für Naturwissenschaftler und Naturwissenschaftlerinnen). Nur für die Wenigsten (12\%) hat das Thema Relevanz (am ehesten Personen aus den Geistes- und Wirtschaftswissenschaften).

\section{Women In Science}

\section{Zufriedenheit und Veränderung der Geschlechterverteilung nach Wissenschaftsbereich}

Knapp die Hälfte (48,3 \%) der Befragten ist zufrieden mit der prozentualen Geschlechterverteilung am Lehrstuhl - besonders Personen aus den Geistes- und Wirtschaftswissenschaften und ein Großteil der befragten Frauen. Unzufrieden mit dieser Situation sind 27,6 \% der Befragten und 24,1\% haben eine indifferente Meinung dazu.

Mit der Geschlechterverteilung in ihrem eigenen Fach ist jedoch nur knapp ein Drittel (32,8 \%) zufrieden; insbesondere die Geistes- und Wirtschaftswissenschaftlerinnen und Geistes- und Wirtschaftswissenschaftler. Die Mehrheit (46,6 \%) ist unzufrieden mit dieser Situation.

Geschlechterverteilung im eigenen Fach wird überwiegend erziehungs- und sozialisationsbedingt gesehen (von über $50 \%$ der Befragten). Mit dieser Einschätzung liegen die Ingenieurwissenschaften im Vergleich zu den anderen Wissenschaftsbereichen sogar noch vor den Geistes- und Wirtschaftswissenschaften (vgl. dazu ausführlicher LeichtScholten 2006a). 43,1\% der Befragten sind der Ansicht, dass diese so- 
wohl durch angeborene Geschlechtsunterschiede als auch durch Sozialisation bedingt ist.

\section{Maßnahmen zur Veränderung der Geschlechterverteilung}

Nur $31 \%$ (vs. $69 \%$ ) der Befragten gaben an, dass am eigenen Institut diesbezügliche Maßnahmen eingesetzt werden (am ehesten in den Ingenieurwissenschaften). Aber nur 22,4 \% würden selbst spezielle Maßnahmen ergreifen, um eine Veränderung der Geschlechterverteilung zu erzielen (vor allem in den Ingenieurwissenschaften). Knapp die Hälfte (48,3 \%) jedoch würde keine Aktivitäten in dieser Hinsicht entwickeln.

An diesem Punkt lässt sich ein Widerspruch feststellen, denn obwohl in der Veränderung der Geschlechterverhältnisse eher Vorteile gesehen werden, wären diese Personen nicht dazu bereit, einen persönlichen Beitrag zu leisten - und dies obwohl es kaum Maßnahmen zur Geschlechtergerechtigkeit am eigenen Institut gibt.

Zur Erzielung einer Veränderung der Geschlechterverteilung werden am häufigsten Maßnahmen befürwortet wie „Mentoringprogramme“ (37,9 \%), „Informationstage speziell für Frauen“ (32,8\%), „Werbung für Studienangebote“ (32,8 \%) und „Spezielle Beratungsangebote für Frauen“ (31\%). Nur die allerwenigsten der Befragten halten „Spezielle Betreuung für Frauen“ (10,3 \%) „Stipendien für Frauen“ (6,9 \%) „Gezielte geschlechtsspezifische Analysen von Berufungsverfahren“ $(5,2 \%)$, „Prämien (Gender Budgetierung) für die Einstellung von Frauen“ (3,4 \%), „Frauen-Quoten bei Neuanstellungen“ (1,7\%), für sinnvoll. (Hier waren Mehrfachnennungen möglich).

\section{Berücksichtigung von Gender-Aspekten in der Lehre}

Zweidrittel $(65,5 \%)$ der Befragten konstatieren ein unterschiedliches Lernverhalten zwischen Männern und Frauen, 15,5 \% habe keine eindeutige Meinung zu dieser Aussage und $19 \%$ können kein unterschiedliches Lernverhalten zwischen den Geschlechtern feststellen.

\section{Berücksichtigung der unterschiedlichen Sozialisation in der Lehre}

$\mathrm{Zu}$ dem Urteil, dass eine unterschiedliche Sozialisation in der Lehre nicht berücksichtigt werden sollte, kommt mehr als die Hälfte (55,2\%) der Befragten (besonders aus dem Bereich Ingenieurwissenschaften). 
Jeweils $22,4 \%$ stimmten dieser These zu oder hatten keine eigene Meinung dazu.

Obgleich die Geschlechterverteilung in den einzelnen Fächern überwiegend sozialisationsbedingt begründet wird (siehe oben) sollen die von den Befragten konstatierten unterschiedlichen Ausgangsbedingungen in der Lehre kaum berücksichtigt werden; mehr als $50 \%$ der Befragten, die die Ursache der Geschlechterverteilung sozialisationsbedingt sehen, sind der Ansicht, dass die unterschiedliche Sozialisation nicht berücksichtigt werden solle. Demzufolge finden geschlechtspezifische Konzepte im didaktischen Konzept der Lehre so gut wie keine Berücksichtigung (96,6 \%). Nur zwei der befragten Personen beziehen derartige Aspekte ein.

\section{Lehrinhalte sind geschlechtsneutral}

Die im eigenen Fachgebiet vertretenen Lehrinhalte werden überwiegend $(86,2 \%)$ als geschlechtsneutral bewertet. Dieser These stimmten alle Befragten aus den Ingenieurwissenschaften und alle befragten Frauen $\mathrm{zu}$.

\section{Sensibilisierung der Lehrenden für das Thema „Gender“}

Knapp die Hälfte (48,2 \%) der Befragten sehen keine Notwendigkeit, Lehrende für das Thema Gender zu sensibilisieren (tendenziell eher Ingenieurwissenschaften). Personen, die einer geschlechtssensiblen Lehre nicht zustimmen sind auch tendenziell eher der Ansicht, dass die Lehrinhalte geschlechtsneutral sind (54\%).

Mehr als ein Drittel (34,5 \%) hingegen haben keine eindeutige Position zu dieser These bezogen. Nur die Wenigsten (17,2\%) halten eine Sensibilisierung für sinnvoll.

\section{Unterschiedliches Technikinteresse der Geschlechter}

Ein unterschiedliches Technikinteresse der Geschlechter wurde von mehr als der Hälfte $(51,7 \%)$ der Befragten angenommen (insbesondere von der Gruppe der Männer zwischen 41-60 Jahren). Nur 12,1 \% sind der Ansicht, dass Männer generell nicht stärker an Technik interessiert sind als Frauen. 


\section{Gender and Science}

\section{Prägung der Disziplin durch das Geschlecht}

Eine geschlechtsspezifische Prägung der Wissenschaft durch ungleiche Geschlechterverteilung wird von mehr als der Hälfte der Befragten (56,9\%) nicht gesehen (eher von Personen über 50 Jahre). Während ein Großteil der Befragten (43,1\%) dazu keine eindeutige Einstellung hatten und $22,4 \%$ eine starke Prägung der Wissenschaft durch das Geschlecht sehen.

\section{Unabhängigkeit der wissenschaftlichen Forschung vom Geschlecht}

Wissenschaftliche Forschung wird von Dreiviertel der Befragten $(75,9 \%)$ als unabhängig vom Geschlecht betrachtet (vor allem von den Ingenieurwissenschaften und Personen zwischen 40 bis 50 Jahre).

Eine indifferente Einstellung zu der These haben 10,3\%, während $13,8 \%$, der Meinung sind, dass die wissenschaftliche Forschung vom Geschlecht abhängig ist.

\section{Zusammenhang zwischen Geschlechtergerechtigkeit und der Qualität von Forschung und Lehre}

Geschlechtergerechtigkeit und die Qualität von Forschung und Lehre wird von der Mehrheit nicht in Zusammenhang gesehen (74,1\%), besonders von den Ingenieurwissenschaften und Personen über 40 bis 60 Jahren. 12,1 \% sehen einen Zusammenhang und 13,8 \% haben eine indifferente Meinung dazu. Demzufolge halten auch fast Zweidrittel (63,8 \%) der Befragten Lehre und Forschung zu Gender-Inhalten für unwichtig.

\section{Integration von Gender-Aspekten in alle Studiengänge}

Eine Integration der Gender-Aspekte in die Studiengänge wird von den meisten der Befragten (56,9 \%) als nicht sinnvoll erachtet (besonders von den Ingenieurwissenschaftlern und Ingenieurwissenschaftlerinnen). Ein Fünftel (20,7 \%) der Befragten aber hält die Integration von GenderAspekten in alle Studiengänge für sinnvoll, während fast ein Viertel $(22,4 \%)$ keine eindeutige Position zu dieser Frage hat. 


\section{Sensibilisierung der Lehrenden für das Thema „Gender“}

Über die Hälfte der Befragten (56 \%) halten eine Integration des Themas Gender übergreifend in allen Studiengängen für nicht sinnvoll ist. Mit geschlechtsspezifischer Didaktik haben sich nur $8 \%$ befasst.

\section{Technikinteresse der Geschlechter}

Die These, dass Männer stärker an Technik interessiert sind, als Frauen wurde von mehr als der Hälfte (52\%) der Ingenieurwissenschaftlern und Ingenieurwissenschaftlerinnen befürwortet. $32 \%$ sind der Ansicht, dass Männer generell nicht stärker an Technik interessiert sind als das andere Geschlecht.

\section{Forschung}

Eine geschlechtsspezifische Prägung der Wissenschaft durch ungleiche Geschlechterverteilung wird mehrheitlich (über $50 \%$ ) nicht gesehen während $22 \%$ eine starke Prägung der Wissenschaft durch das Geschlecht sehen und $28 \%$ keine eindeutige Position dazu haben.

Wissenschaftliche Forschung wird vielmehr von $84 \%$ der Befragten als unabhängig vom Geschlecht betrachtet (am ehesten die Ingenieure und Ingenieurinnen). Demzufolge halten fast 2/3 Lehre und Forschung zu Gender-Inhalten für unwichtig. Auch ein Zusammenhang zwischen Geschlechtergerechtigkeit und der Qualität von Forschung und Lehre wird von der Mehrheit nicht gesehen.

\section{Zusammenfassung}

1. Allgemein lässt sich festhalten, dass sich das Verständnis von Geschlechtergerechtigkeit primär auf die numerische Verteilung der Geschlechter in den Disziplinen bezieht.

2. Eine ausgeglichene Geschlechterverteilung wird zwar von einem Großteil der Befragten begrüßt, generell wird aber kein Zusammenhang zwischen Geschlechtergerechtigkeit und der Qualität von Forschung und Lehre gesehen.

3. Bisher findet eine Integration von Gender-Aspekten in Forschung und Lehre nicht statt und wird von einem Großteil der Befragten auch für die Zukunft nicht gewünscht.

4. Es gibt keinen „gender-fortschrittlichen“ Fachbereich. Neben der zu erwartenden Offenheit von Geistes- und Wirtschaftswissenschaftlern 
gegenüber der Thematik wird auch von $1 / 3$ der Befragten aus den Ingenieurwissenschaften die Integration von Gender-Aspekten in Forschung und Lehre als sinnvoll erachtet.

5. Es gibt keinen Zusammenhang zwischen Gender-Awareness und Geschlecht.

\section{Fazit}

Was bedeuten diese Ergebnisse bezügliche der Eingangs formulierten Fragen? Wie viel Gender ist im Mainstream der Wissenschaften?

Aufgrund der geringen Beteiligung an der Befragung können die Ergebnisse nur als Tendenzen verstanden werden. Da aber auch davon auszugehen ist, dass die geringe Beteiligung auch mit einem geringen Interesse am Thema zusammenhängen können und sich dem zufolge vorwiegend diejenigen an der Befragung beteiligt haben, die der Thematik prinzipiell aufgeschlossen gegenüber stehen, liefern die Ergebnisse der Befragung sicherlich eher eine positive Einschätzung der Situation.

Die Ergebnisse der Befragung zeigen die unterschiedliche Relevanz, die Gender-Aspekten im Mainstream der Wissenschaften zugemessen wird, bis zur vollständigen Negierung der Gender-Dimension für die Wissenschaft. Sie macht die unterschiedlichen „Gender Blicke“, aber auch die vielen gender-blinden Flecken im Gesichtsfeld der Wissenschaft sichtbar. Um das Konzept des Gender Mainstreaming an Hochschulen umzusetzen, und so Frauen und Männern gleichermaßen in Forschung und Lehre, bei der Entwicklung von Forschungsprojekten und Generierung von Forschungsergebnissen zu unterstützen, ist die Hochschule gefordert, gerade Professorinnen und Professoren für die Umsetzung des Gender Mainstreaming Konzeptes zu gewinnen.

\section{Handlungsempfehlungen}

Ausgehend von den Ergebnissen der Befragung lassen sich kurz- und mittelfristige Handlungsempfehlungen für die Hochschule ableiten, um das Konzept des Gender Mainstreaming in den unterschiedlichen Bereichen der Hochschule umzusetzen.

\section{Kurzfristige Handlungsempfehlungen}

1. Entwicklung eines detaillierteren Genderprofils mit klarer Definition von Gender Mainstreaming als Reorganisationsansatz für alle Hand- 
lungsbereiche der Hochschule. Verstärkte Öffentlichkeitsarbeit zum Konzept des Gender Mainstreaming und seiner Bedeutung in und für die Hochschule nach innen und nach außen.

2. Verstärkte Öffentlichkeitsarbeit zum Konzept des Gender Mainstreaming und seiner Bedeutung in und für die Hochschule nach innen und nach außen (z.B. Ringvorlesungen zum Thema, Plakate, Flyer).

3. Initiierung und Unterstützung von Projekten die Gender-Aspekte in der Forschung berücksichtigen (vgl. Fraunhofer Gesellschaft).

4. Anstoß und Unterstützung von Fachbereichen zur Einbeziehung von Gender-Aspekten in die Lehre (Fortbildungen).

5. Weiterbildungsangebot für Lehrende zu gendergerechter Lehre.

6. Schaffung von finanziellen Anreizen für best practice Projekte.

\section{Mittel- und langfristige Handlungsempfehlungen}

Um die eingangs im Leitbild der RWTH formulierten Ziele zu erreichen und damit die Fähigkeiten von Frauen und Männern gleichermaßen in Forschung und Lehre, bei der Entwicklung von Forschungsprojekten und Generierung von Forschungsergebnissen zu unterstützen, ist die Hochschule gefordert, gerade Professorinnen und Professoren für die Umsetzung des Gender Mainstreaming Konzeptes zu gewinnen.

Die Hochschule hat mit der Formulierung ihres Leitzieles und des Genderprofils erste Schritte getan. Nun gilt es beides im Hochschulalltag im Sinne der top-down-Strategie gemeinsam mit Professorinnen und Professoren umzusetzen. Soll Gendergerechtigkeit als Qualitätsmerkmal der Hochschulentwicklung eingestuft werden, ist es notwendig die Umsetzung von Gender Mainstreaming innerhalb der Hochschule in einen entsprechenden institutionellen Rahmen einzubetten. Soll das beschriebene Leitbild mehr sein als eine Vision, so gilt es, die Hochschule als gesamte Organisation auf allen hierarchischen Ebenen im Hinblick auf Geschlechtergerechtigkeit zu reorganisieren und die Geschlechterfrage auch als wesentliches Kriterium für die Entwicklung von Forschungsfragen und Forschungsprojekten zu betrachten.

Beispielhaft lässt sich hier das seit November 2005 an der Universität Duisburg-Essen entwickelte Zentrum für Hochschul- und Qualitätsentwicklung nennen, dessen vier Aufgabenbereiche Hochschuldidaktik, ELearning, Gender Mainstreaming und Finanzförderung und Evaluation umfassen. Die enge Vernetzung und Kooperation der vier Geschäftsbereiche ermöglicht die Umsetzung einer geschlechtergerechten Hochschule und kann Impulse in die unterschiedlichen Handlungsfelder ge- 
ben (Personalentwicklung, Organisationsentwicklung, Gleichstellungspolitik, Lehre und Studium, Forschung) (vgl. Klees-Möller/Rompeltien in diesem Band).

Es ist unabdingbar, eine starke und unabhängige Struktur zu schaffen, von der ausgehend Impulse gegeben werden können, um das Konzept des Gender Mainstreaming auf den unterschiedlichsten Handlungsfeldern der Hochschule umzusetzen und die Gender-Perspektive in Lehre und Forschung ebenso wie in der Personal- und Organisationsentwicklung der Hochschule zu berücksichtigen.

Auch über die Schaffung einer direkt dem Rektorat zugeordneten Stabstelle könnte eine derartige Einheit geschaffen werden, die sowohl top-down, wie z.B. gendergerechte Personalentwicklungskonzepte für Professorinnen und Professoren, als auch in Kooperation mit den Fakultäten bottom-up-Konzepte und Strategien entwickeln kann, um langfristig das Konzept des Gender Mainstreaming als Reorganisationsprozess der Hochschule umzusetzen.

Soll das beschriebene Leitbild mehr sein als eine Vision, so gilt es die Hochschule als gesamte Organisation auf allen hierarchischen Ebenen im Hinblick auf Geschlechtergerechtigkeit zu reorganisieren und die Geschlechterfrage auch als wesentliches Kriterium für die Entwicklung von Forschungsfragen und Forschungsprojekten zu betrachten.

Mit der Einbindung von Gender Mainstreaming in die Hochschul- und Qualitätsentwicklung können Impulse in die unterschiedlichsten Handlungsfelder der Hochschule gegeben werden und damit deutlich gemacht werden, dass das Konzept weit mehr beinhaltet als Frauenförderung, der „Gender Blick“ kann vielmehr eine qualitative Bereicherung für alle Beteiligten sein.

\section{Ausblick}

Eine Chance, das Konzept des Gender Mainstreaming in Wissenschaft und Forschung integrieren zu können, bietet der umfassende Transformationsprozess, in dem sich das deutsche Hochschulsystem gegenwärtig befindet. Durch die Reformulierung des Verhältnisses von Staat und Hochschule und den Hochschulreformprozess werden die Hochschulen der Zukunft zu „entrepreneurial universities“, die von Wissensmanagern geführt werden. So trägt die Exzellenzinitiative in Deutschland sicherlich dazu bei, alte Strukturen innerhalb der Hochschulen zu überdenken und neuen visionären Konzepten Raum zu geben und damit auch Gender-Aspekte verstärkt im Reformprozess zu berücksichtigen. 


\section{Literatur}

Bereswill, Mechthild (2004): „Gender“ als neue Humanressource? Gender Mainstreaming und Geschlechterdemokratie zwischen Ökonomisierung und Gesellschaftskritik. In: Gender Mainstreaming. Konzepte - Handlungsfelder - Instrumente, hg.v. Michael Meuser und Claudia Neusüß, Bundeszentrale für Politische Bildung, Bonn, S. 52-70.

Competenzzentrum Frauen in Wissenschaft und Forschung (CEWS) (Hrsg.) (2005): Hochschulranking nach Gleichstellungsaspekten. Erste Fortschreibung, Bonn.

Degele, Nina (2000): Anpassen oder unterminieren: Zum Verhältnis von Gender Mainstreaming und Gender Studies. In: Freiburger FrauenStudien, S. 1-13.

European Commission (2004): Gender and Excellence in the Making, Luxemburg, http://europa.eu.int/comm/research/science-ociety/pdf/ bias_brochure_final_en.pdf.

Leicht-Scholten, Carmen (2006a): Wie viel gender ist im mainstream der Wissenschaft? In: Soziale Technik, hg.v. IFZ Interuniversitäres Forschungszentrum für Technik, Arbeit und Kultur (Ed.), 4/2006, Universität Graz, S. 19-21.

Leicht-Scholten, Carmen (2006b): Gender and Science - Perspektiven für die Wissenschaft. In: ADA-MENTORING, 16. Ausgabe, Dortmund, S. 13-16.

Leitbild der RWTH www-zhv.rwth-aachen.de/zentral/dez5_leitbild.htm.

Meuser, Michael (2004): Zum Verhältnis von Geschlechterforschung und Geschlechterpolitik. In: Gender Mainstreaming. Konzepte Handlungsfelder - Instrumente, hg.v. Michael Meuser/Claudia Neusüß, Bonn, S. 322-336.

Zahlenspiegel der RWTH Aachen (2005): http://www-zhv.rwthaachen.de/zentral/dez6_publikationen_zahlenspiegel_05.pdf.

Zielvereinbarungen II zwischen dem Ministerium für Wissenschaft und Forschung des Landes Nordrhein Westfalen und der RWTH Aachen vom 21.01.2005. 



\section{Gender im Mainstream der Hochschulent- wicklung. Institutionalisierung von Frauen- förderung und Gender Mainstreaming am Beispiel MEDUSE}

RENATE KLEES-MÖLLER UND BÄRBEL ROMPELTIEN

Das Konzept des Gender Mainstreaming ist an Hochschulen vielfach lediglich auf der Ebene der Rhetorik verankert, das Verhältnis zur Frauenförderung nicht selten ungeklärt. Im vorliegenden Beitrag wird ein konkretes Beispiel für die institutionelle Umsetzung von GM und Frauenförderung im Hochschulmanagement vorgestellt, aus dem sich Hinweise für eine erfolgreiche Gender-Strategie ablesen lassen.

\section{Vorbemerkung}

Seit der Amsterdamer Vertrag (1. Mai 1999) alle Mitgliedsstaaten der EU zu einer aktiven Gleichstellungspolitik im Sinne des Gender Mainstreaming verpflichtet, ist Gender Mainstreaming bundes- und landespolitisch als Leitlinie etabliert und auch als rechtliche Vorgabe für die Hochschulen verpflichtend. Gender Mainstreaming wird dabei so verstanden, dass alle Maßnahmen und Entscheidungen unter dem Aspekt ihrer Auswirkungen auf die Geschlechterverhältnisse im jeweiligen Bereich analysiert und bewertet und gegebenenfalls auch entsprechend ausgestaltet werden. Frauenförderung erscheint in dieser Perspektive als eine mögliche Umsetzung des Gender Mainstreaming - sofern nämlich bei der Genderanalyse gravierende Benachteiligungen von Frauen sichtbar werden. Frauenförderung wird aber generell auch als grundsätzlich andersartiger Ansatz der Gleichstellungspolitik beschrieben (vgl. BMFSFJ 2003: 30ff.). Während Frauenförderung als zielgruppenorientierter Politikansatz gesehen wird, der institutionell als Auftrag spezifischen Akteuren zugewiesen ist, den Frauen- oder Gleichstellungsbeauf- 
tragten insbesondere, wird Gender Mainstreaming als strukturell ausgerichteter Politikansatz hervorgehoben, der zwar bei spezifischen Benachteiligungen nicht so direkt greife, dafür aber nachhaltigere und weiterreichende Wirkungen durch Einbeziehung aller innerinstitutionellen Akteure und durch genderorientierte Betrachtung aller Handlungsbereiche erreichen könne (vgl. ebd.).

In der Praxis der Gleichstellungspolitik an Hochschulen wird das prägnant formulierte Konzept des Gender Mainstreaming, das ja auch einen Rahmen für Frauenförderung bilden soll, bisher unzureichend, oder jedenfalls sehr uneinheitlich umgesetzt. Vielfach ist Gender Mainstreaming lediglich auf der Ebene der Rhetorik verankert; die erforderliche Gender-Kompetenz fehlt. Auch sind die Befürchtungen, Gender Mainstreaming könne die bisherige Politik der Frauenförderung schwächen, keineswegs ausgeräumt. Mit anderen Worten: Sollen beide Politikansätze sich wechselseitig unterstützen und zusammenwirken, um Fortschritte in der Gleichstellung der Geschlechter an der Universität zu erreichen, so müssen institutionelle Regelungen Frauenförderung und Gender Mainstreaming in ein klares Verhältnis zueinander rücken. Im vorliegenden Beitrag wird ein konkretes Beispiel für die institutionelle Umsetzung von Gender Mainstreaming und Frauenförderung im Hochschulmanagement vorgestellt. Die Universität Duisburg-Essen hat mit der Einrichtung des Geschäftsbereichs Frauenförderung und Gender Mainstreaming im Zentrum für Hochschul- und Qualitätsentwicklung im Kontext der Einführung des modernen Instrumentariums der Hochschulsteuerung eine durchgehende Gender-Strategie aufgelegt, die sowohl die gezielte Frauenförderung wie das Gender Mainstreaming umfasst. Auch wenn die Ansatzpunkte dieser Entwicklung in spezifischer Weise mit der Situation der Neuordnung der Universität zusammenhängen, die als Fusion zweier Vorgänger-Universitäten 2003 neu gegründet wurde, so lassen sich doch einige allgemeine Hinweise ableiten, welche Merkmale eine Gender Mainstreaming-Strategie erfolgreich machen können.

\section{Gender Mainstreaming in der Hochschulentwicklungsplanung}

Das Rektorat der Universität Duisburg-Essen hat für die Hochschulentwicklungsplanung das Instrument der internen Ziel- und Leistungsvereinbarungen (ZLV), die auf der Grundlage von Entwicklungs- oder Strukturgesprächen zwischen Hochschulleitung und den Fachbereichen und Einrichtungen abgeschlossen werden, eingeführt (Zechlin 2003; Universität Duisburg-Essen). Die Leitlinie des Gender Mainstreaming 
wird dabei durchgängig auf der Prozessebene mitgeführt (siehe Abbildung 1: Gender Mainstreaming im Hochschulmanagement).

Der Prozess der ZLV startet mit der Formulierung von Entwicklungszielen für zentrale Entwicklungs- und Handlungsfelder der Universität, wobei die gleichstellungspolitischen Ziele zwischen dem Rektorat und der Gleichstellungsbeauftragten abgestimmt werden. Die Gleichstellung wird als Querschnittskategorie innerhalb der Kernbereiche Studium und Lehre, Forschung und wissenschaftlicher Nachwuchs berücksichtigt. Auf der Management-Ebene wird Gender unter dem Aspekt von vorhandenen oder noch benötigten Ressourcen und Kompetenzen betrachtet.

Der Prozess der Entwicklungsplanung mit ZLV umfasst drei Schritte: Am Anfang stehen datenbasierte Analysen der Situation der Fachbereiche in den angesprochenen Kern- bzw. Leistungsbereichen. Die IstAnalyse (Schritt I) soll den gegenwärtigen Entwicklungsstand in den genannten Leistungsbreichen darlegen, die Zielentwicklung (Schritt II) soll im Abgleich zwischen Zielperspektiven des Rektorates und Fachbereichszielen realistische und zugleich entwicklungsförderliche Zielsetzungen generieren, und die darauf Bezug nehmende Maßnahmen- und Ressourcenplanung (Schritt III) soll die Umsetzungsschritte, die zur Zielerreichung ergriffen werden, konkretisieren. Der Prozess wird mit dem Abschluss einer formellen Ziel- und Leistungsvereinbarung abgeschlossen, die die Ziele sowie die zu ergreifenden Maßnahmen und die bereitzustellenden Ressourcen (Mittel, Dienstleistungen und andere Ressourcen) aufführen.

Der so gestaltete Prozess der Hochschulentwicklungsplanung rückt Gleichstellung sowohl als Zielkategorie wie als Qualitätskriterium in den Kernbereich der Planung. Die Struktur dieses Prozesses entspricht im Übrigen ersichtlich dem Vorgehen zur Erstellung von Frauenförderplänen, die bereits durch das Landesgleichstellungsgesetz (LGG) als Instrument der Planung für Fortschritte in der Gleichstellung von Frauen und Männern vorgegeben sind. Deutlich wird, dass in der Prozesslogik der ZLV die Hochschulentwicklungsplanung auf Fachbereichsebene und die Aufstellung der Frauenförderpläne parallel verlaufen und somit leicht miteinander zu koppeln sind. Dies trifft auch für das anschließende Monitoring und die Bewertung der Zielerreichung am Ende der Laufzeit zu. Wenn auch die Koppelung noch nicht flächendeckend erreicht wurde, so sind erstmals auf der Basis der für die Entwicklungsgespräche erstellten Leistungsdaten Frauenförderpläne erstellt worden.

Die zwischen Rektorat und Fachbereichen getroffenen Zielvereinbarungen umfassen, je nach Ausgangslage auch Maßnahmen zur Frauenförderung, für die in den bisherigen Vereinbarungsrunden finanziell ein 
beachtliches Volumen mobilisiert werden konnte. Wichtige Entwicklungen in den Fachbereichen konnten angestoßen werden. Inhaltlich waren die gleichstellungsbezogenen Zielvereinbarungen 2005 und 2006 dem Schwerpunkt der Förderung des weiblichen wissenschaftlichen Nachwuchses, insbesondere der Förderung der Promotion von Frauen, gewidmet. Bei den Entwicklungsgesprächen wurde deutlich, dass in vielen Fachbereichen ein großer Beratungsbedarf hinsichtlich möglicher Ansatzpunkte und praktischer Beispiele (good-practice Modelle) für Fortschritte in der Gleichstellung besteht. Explizit wurde ein Unterstützungsbedarf für die Entwicklung von Gender-Kompetenz benannt. Hier ist die Schnittstelle zwischen den Zielvereinbarungen als Steuerungsinstrument und der Implementierung von Gender Mainstreaming als institutionelle Dienstleistung für die Universität einerseits, zwischen Gender Mainstreaming als Dienstleistung und der Integration von GenderKriterien in weitere Steuerungsinstrumente der Hochschule, wie die Budgetierung, das Controlling, die Qualitätssicherung sowie die Personal- und Organisationsentwicklung. 


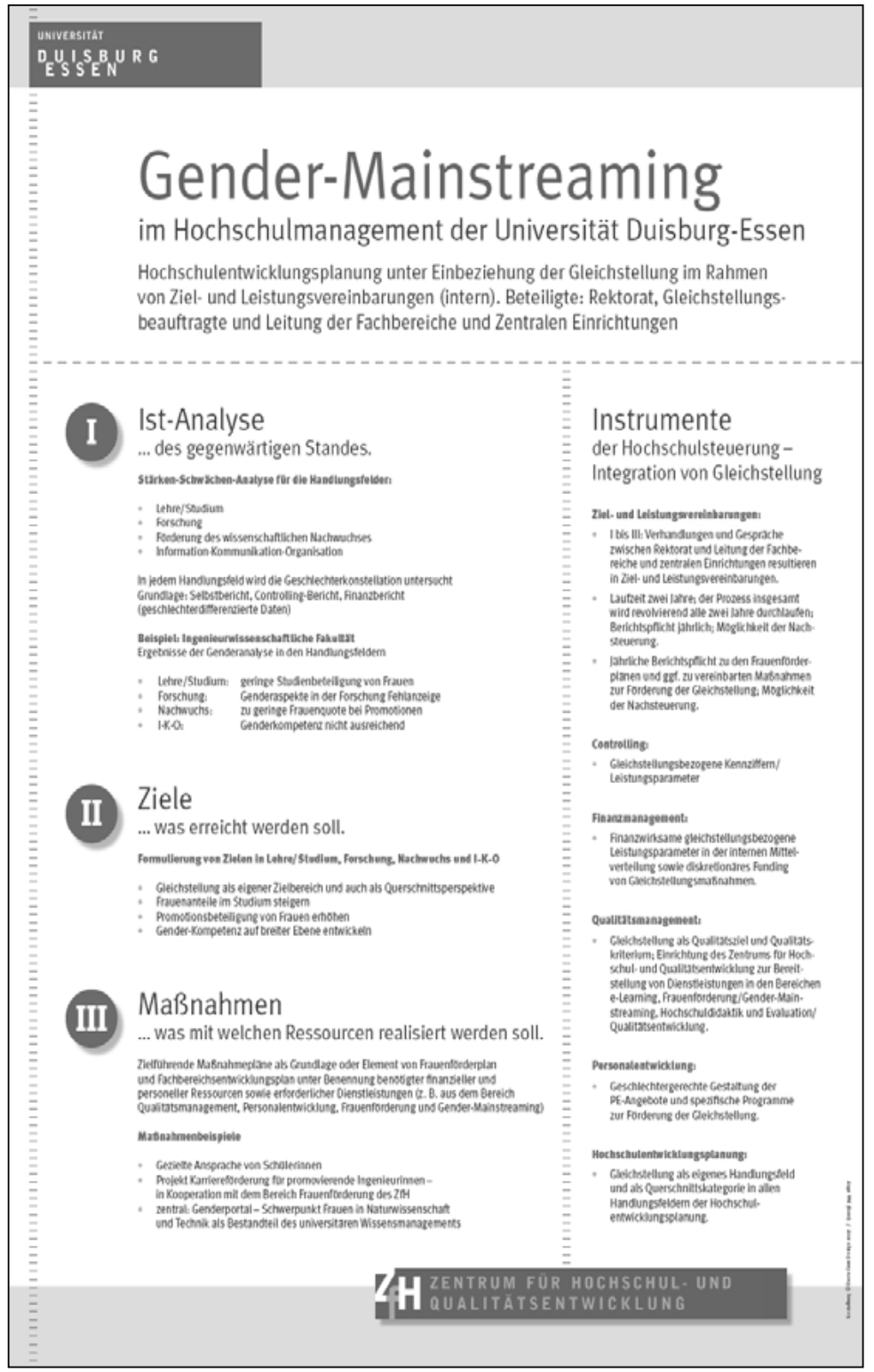

Quelle: Zentrum für Hochschul- und Qualitätsentwicklung 


\section{Implementierung von Frauenförderung und Gender Mainstreaming als wissenschaftliche Dienstleistung}

Die Universität Duisburg-Essen (UDE) hat die Förderung der Chancengleichheit bereits in ihrer Gründungsphase als relevanten Zielbereich der Hochschulentwicklung und als Qualitätskriterium für das Hochschulmanagement benannt und mit einer Reihe ineinander greifender Maßnahmen und Entscheidungen umgesetzt. Die grundlegenden Prozesse des Hochschulmanagements wurden unter Einbeziehung von Gleichstellungsaufgaben reformuliert. Die UDE hat damit eine Vorreiterrolle übernommen, was die systematische Modernisierung der Managementprozesse unter Einbeziehung der Gleichstellung anbelangt mit der Besonderheit, dass zur Unterstützung bestimmte Aufgabenbereiche des Hochschulmanagement eine eigene zentrale Einrichtung mit Dienstleistungsauftrag geschaffen wurde.

Abbildung 2: Organisationsaufbau des Zentrums für Hochschul- und Qualitätsentwicklung der Universität Duisburg-Essen

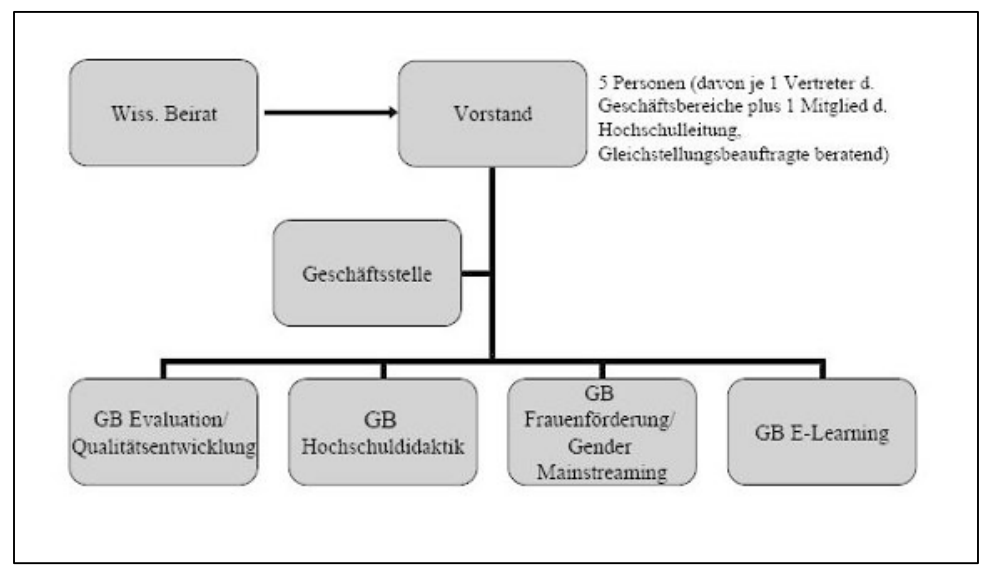

Quelle: Zentrum für Hochschul- und Qualitätsentwicklung 11/06, internes Organigramm

Die Überführung von der Projekt- und Prozess-Ebene auf die Strukturebene der Universität wurde mit der Gründung des Zentrums für Hochschul- und Qualitätsentwicklung (ZFH) vollzogen. Das ZFH weist vier Geschäftsbereiche auf, und zwar den Geschäftsbereich Evaluation/ Qualitätssicherung, den Geschäftsbereich Hochschuldidaktik, den Ge- 
schäftsbereich Frauenförderung/Gender Mainstreaming und den Geschäftsbereich E-Learning. Das gesamte Zentrum hat den Auftrag, durch wissenschaftliche Dienstleistungen Prozesse der Hochschul- und Qualitätsentwicklung in den Bereichen Lehre und Forschung sowie Management und Services zu unterstützen (siehe Abbildung 2 Organisationsaufbau des Zentrums für Hochschul- und Qualitätsentwicklung der Universität Duisburg-Essen). Für die Einrichtung des Geschäftsbereichs Frauenförderung/Gender Mainstreaming wurden die bislang im Projektzentrum MEDUSE durchgeführten Projekte und Programme, im Wesentlichen zentriert um Mentoring-Projekte und Programme zur Karriereförderung für unterschiedliche Zielgruppen, zusammengeführt und mit dem zusätzlichen Auftrag versehen, ein Dienstleistungsangebot „Umsetzung von Gender Mainstreaming“ zu entwickeln .

Karriereförderung für Frauen als Teil der akademischen Pesonalentwicklung und die Umsetzung der Gender Mainstreaming-Strategie in den zentralen Handlungsfeldern der Universität sind so als wesentliche Faktoren der Organisations- und Personalentwicklung der Universität Duisburg-Essen eingeschätzt und zentral platziert worden. Damit wurde ein wesentliches strukturelles Element für die Umsetzung des Gender Mainstreaming geschaffen, ein „starker Akteur“, der sichtbare Kompetenzen und eine eigene Zuständigkeit für die Querschnittsaufgabe Gender Mainstreaming besitzt. Dieser Akteur ist einerseits eingebunden in eine top-down-Strategie des Gender Mainstreaming, andererseits ist er dafür prädestiniert, über Dienstleistungsangebote die Anforderungen aufzugreifen, die bottom-up von den Fachbereichen artikuliert werden, und sie in gemeinsamen Entwicklungsprojekten produktiv zu bearbeiten. Da der Geschäftsbereich die Querschnittsaufgabe Gender Mainstreaming auch innerhalb des Zentrums selbst wahrnimmt, ist gesichert, dass auch in den Aktivitäten der anderen Geschäftsbereiche, die strategische Entwicklungsfelder der Universität betreffen, Gender-Gesichtspunkte einbezogen werden.

Die Aufgabenfelder und die Arbeitsweise des Geschäftsbereiches Frauenförderung/Gender Mainstreaming (MEDUSE) werden im Folgenden näher beschrieben.

\section{Aufgaben und Aufgabenwahrnehmung im Geschäftsbereich Frauenförderung/Gender Mainstreaming}

Im Geschäftsbereich Frauenförderung/Gender Mainstreaming ist über langjährige Projekterfahrung und Durchführung von Maßnahmen in $\mathrm{Zu}$ - 
sammenarbeit mit der Gleichstellungsbeauftragten eine umfassende Kompetenz in der Karriereförderung für Frauen unterschiedlicher Qualifikations- und Karrierestufen vorhanden, die nun perspektivisch in ein Programm zur gendersensiblen akademischen Personalentwicklung einmünden kann.

Bislang wurden Mentoring-Programme für Frauen auf unterschiedlichen Qualifizierungsstufen realisiert. Die ganze Bandbreite der für eine Universität relevanten Zielgruppen, von Schülerinnen bis zu Nachwuchswissenschaftlerinnen, konnte erreicht werden. Alle MentoringProgramme umfassen als Bausteine eine bilanzierende Karriereberatung (1), die Vermittlung einer Mentorship-Beziehung (2), ein begleitendes Qualifizierungsprogramm (3) sowie Vernetzungsangebote (4). Alle Programmbausteine werden für die jeweils angesprochene Zielgruppe spezifisch ausgestaltet. Über eine begleitende Evaluation wird eine kontinuierliche Verbesserung und Qualifizierung der Programme gesichert. Die begleitenden Qualifizierungsangebote im Bereich der Schlüsselqualifikationen sind inzwischen auch unabhängig von den Mentoring-Programmen im Rahmen der gestuften Studiengänge als Module verfügbar.

Die Mentoring-Programme sollen hier im Einzelnen nicht dargestellt werden, da Mentoring-Ansätze inzwischen weit verbreitet sind und ihre Leistungsfähigkeit bei der Bewältigung von Statuspassagen und Entwicklungsschritten unbestritten ist (Löther 2003). Hervorzuheben sind aber einige Besonderheiten, die für das Angebot der Universität Duisburg-Essen charakteristisch sind (Klees-Möller 2006; Klees-Möller/Petersen/Schönborn 2007).

\section{Mentoring für Studentinnen und Absolventinnen mit Migrationshintergrund}

Dies ist einmal die Entwicklung von Programmen für Studentinnen und Absolventinnen mit Migrationshintergrund auf der Grundlage eines Diversity-Konzeptes. Zielsetzung der interkulturellen Programmlinie für Studentinnen mit Migrationshintergrund ist, sie bereits zu diesem frühen Zeitpunkt in ihrer beruflichen Karriereplanung zu begleiten, zu fördern und ihnen dabei zu ermöglichen, ihre verdeckten Ressourcen und Potenziale, die sie aufgrund ihrer Biographie mitbringen, zu entdecken, weiter zu entwickeln und für ihre berufliche Entwicklung nutzbar zu machen. Das Programm „Mentoring Diversity“ für Akademikerinnen mit Migrationshintergrund (gefördert mit Mitteln der EU und des Landes NRW) unterstützt den beruflichen Einstieg und Aufstieg. Es gibt nicht nur den Teilnehmerinnen Gelegenheit, ihre spezifischen Kompetenzen, u.a. interkulturelle und sprachliche Kompetenzen, zu vertiefen, sondern richtet 
sich auch darauf, über Kontakte und Kooperationen mit Unternehmen und Organisationen der Region den Zugang zu den Thematiken „Interkulturalität und Arbeitswelt“ sowie „Gender“ und „Diversity“ für alle Beteiligten zu eröffnen. Mentoring erweist sich im Kontext dieser Programmlinien als Instrument einer vorgezogenen Personalentwicklung insbesondere für Klein- und Mittelunternehmen (Rompeltien 2004).

\section{Personalentwicklung für den wissenschaftlichen Nachwuchs}

Die Förderung des wissenschaftlichen Nachwuchses zählt zu den wesentlichen Entwicklungszielen der UDE. Es gilt, mehr Frauen zur Promotion zu motivieren und Strukturen zu schaffen, die einer größeren Anzahl Nachwuchswissenschaftlerinnen, die im Wissenschaftsfeld immer noch unterrepräsentiert sind, eine schnellere und erfolgreiche Promotion ermöglichen. Zugleich verfolgt die Universität das Konzept des „strukturierten Promovierens“, um sowohl die Qualität der Promotionsbetreuung zu verbessern wie auch die Selbständigkeit und die Selbstorganisation der Promovierenden zu stärken. Eine stärkere Systematisierung der ersten Stufe der selbständigen wissenschaftlichen Arbeit soll den zügigen Abschluss unterstützen und durch intensive Vernetzung weitere Wissensressourcen erschließen. Die Universität fördert mit internen Mitteln eine Reihe von Vorhaben in den Fachbereichen, die in dieser Zielrichtung arbeiten. Der Geschäftsbereich Frauenförderung/ Gender Mainstreaming unterstützt diese Aktivitäten durch folgende Maßnahmen der Personalentwicklung, die teilweise exklusiv für Frauen, teilweise aber auch für beide Geschlechter angeboten werden. Die Sinnhaftigkeit struktureller Veränderungen der Promotionsphase, die geeignet sind, die Promotionsbeteiligung von Frauen und vom wissenschaftlichen Nachwuchs überhaupt, zu verbessern, wird dabei in der Universität als gegeben unterstellt.

\section{Promotionsorientierungs- und Begleitprogramm}

Das Projekt „Promovieren - Eine Perspektive für Frauen“ ist als fachübergreifendes modulares Beratungs-, Vernetzungs- und Schulungsangebot konzipiert und mit großer Resonanz durchgeführt worden. Im Rahmen der Herbstakademie für Promovierende 2006 wurden viele Angebote erstmals auch für männliche Interessenten geöffnet.

In Abstimmung mit den Koordinatorinnen fachspezifischer Förderungsprogramme für Frauen wurde das Projekt im Rahmen interner Innovationsprojekte weiter entwickelt und umfasst folgende Module, die 
die fachspezifischen Angebote zur Promovierendenförderung ergänzen sollen.

Abbildung 3: Module zur Promovierendenförderung

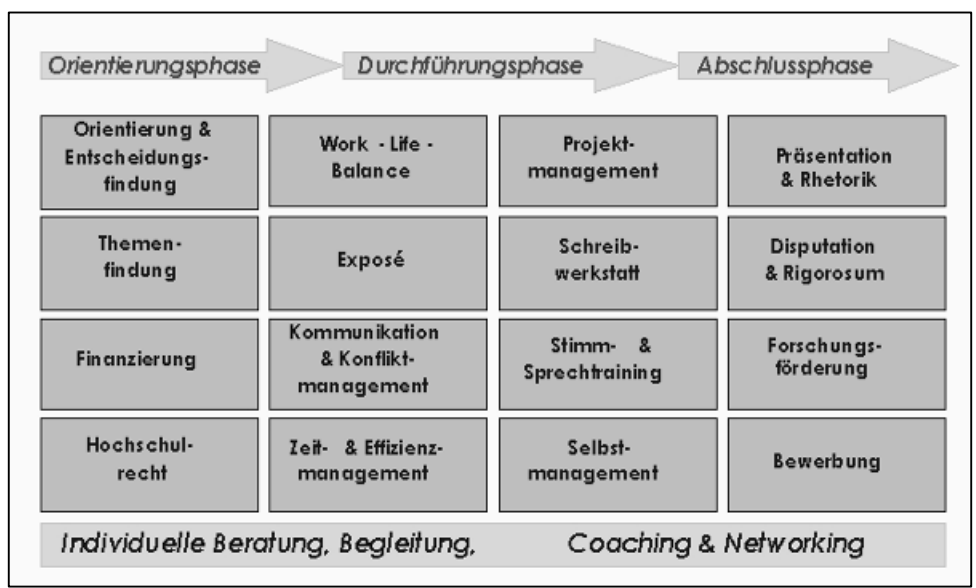

Quelle: ZfH, Frauenförderung/Gender Mainstreaming 10/06, interne Grafik

Zur weiteren gegenseitigen Unterstützung können sich die Promovierenden in selbst organisierten Kleingruppen vernetzen. Diese werden durch zusätzliche moderierte Austauschtreffen sowie durch Einzelberatung professionell begleitet.

\section{Mentoring-Programm für Promovendinnen der Ruhrgebietshochschulen (mentoring ${ }^{3}$ )}

Es handelt sich um ein Kooperationsprojekt, welches mit den Nachbaruniversitäten Bochum und Dortmund in den Fächergruppen Naturwissenschaften (Koordinationsschwerpunkt der Ruhr-Universität Bochum), Ingenieurwissenschaften (Koordinationsschwerpunkt der Universität Dortmund) und Geistes- und Bildungswissenschaften (Koordinationsschwerpunkt der UDE) durchgeführt wird. Hierdurch wird eine optimale Nutzung vorhandener Ressourcen erreicht und eine neue kooperative Kultur generiert.

Fortgeschrittenen Doktorandinnen wird der Austausch mit erfahrenen Professorinnen und Professoren und habilitierten Wissenschaftlerinnen und Wissenschaftlern der drei kooperierenden Hochschulen und außeruniversitären Forschungseinrichtungen vermittelt, wahlweise im one- 
to-one- oder als Gruppen-Mentoring. Im Sinne des Cross-MentoringGedankens fungieren diese erfahrenen Wissenschaftlerinnen und Wissenschaftler als Mentorinnen und Mentoren und geben in Gesprächen wichtiges Handlungswissen über Strukturen und Prozesse im Wissenschaftsbetrieb weiter, was den Nachwuchswissenschaftlerinnen die Positionierung in der Scientific Community erleichtert. Durch Einblicke in universitäre Laufbahnen und Aufstiegsmechanismen können sie individuelle Perspektiven einer Wissenschaftskarriere entwickeln. Mentoring fördert das Eigenengagement der Teilnehmerinnen und ihre individuelle Persönlichkeitsentwicklung.

Ein professionelles Seminarprogramm vermittelt fachübergreifende Schlüsselkompetenzen und qualifiziert die Promovendinnen im Sinne des Personalentwicklungsgedankens. Zusätzlich werden in einer Reihe wissenschaftlicher Symposien Veränderungen im Wissenschaftssystem verfolgt und Perspektiven entwickelt: Renommierte Referentinnen und Referenten erörtern Themen wie „Weibliche Exzellenz“, „Internationalisierung“ und „Wissenschaftskarriere im Wandel“ vor dem Hintergrund der Geschlechterperspektive.

Im Rahmen dieses Projektes konnte eine beachtliche Zahl von Hochschullehrerinnen und Hochschullehrern in der mentoriellen Funktion erreicht werden. Im Mentoring-Prozess entwickeln die Beteiligten durch die intensive Auseinandersetzung mit Gender-Fragestellungen in Bezug auf Karriereanforderungen und -bedingungen in der Wissenschaft eine Sensibilität für Disparitäten und Diskriminierungen in der Situation von Männern und Frauen im wissenschaftlichen Nachwuchs - und günstigenfalls auch die Motivation, einen Beitrag zu einer anderen Wissenschaftskultur leisten zu wollen. Insbesondere Fragen zur Karriere- und Lebensplanung, die an der männlich geprägten Arbeitskultur im Wissenschaftsfeld orientiert sind und die Entgrenzung von Privatem und Beruflichem betreffen, finden hierdurch verstärkt Beachtung. Die professoralen Mentorinnen und Mentoren werden auf diesem Wege zu wichtigen Türöffnern für Gender Mainstreaming in den Fachbereichen.

\section{Karriereförderungsprogramm für Postdocs in der Medizin (MediMent)}

Im Auftrag der Medizinischen Fakultät führt der Geschäftsbereich Frauenförderung/Gender Mainstreaming ein Seminarprogramm zur Karriereförderung für Wissenschaftlerinnen in der Medizin durch. Aufgrund der positiven Erfahrungen wurde das Seminarprogramm durch ein Mentoring-Programm für promovierte Wissenschaftlerinnen in der Medizin erweitert. Es richtet sich vorrangig an Postdoktorandinnen, die eine wis- 
senschaftliche Karriere in der Klinik und/oder Grundlagenforschung anstreben. Habilitierte Wissenschaftlerinnen und Professorinnen des Essener Universitätsklinikums begleiten Nachwuchswissenschaftlerinnen aus verschiedenen Bereichen der Medizinischen Fakultät auf den weiteren Schritten in Richtung ihrer Habilitation oder Facharztausbildung (siehe MediMent).

Die Darstellung der Aufgabenwahrnehmung im Bereich der akademischen Personalentwicklung zeigt, in welcher Weise eine Gender Mainstreaming-Strategie in diesem Handlungsfeld wirksam werden kann. Der Geschäftsbereich realisiert Projekte und Programme mit großer Ausstrahlung, wie die Herbstakademie, das Medizin-Mentoring oder das kooperative Promovendinnen-Mentoring der Ruhrgebietshochschulen. Die professionelle Kompetenz wird so überzeugend demonstriert. Zugleich greift der Geschäftsbereich Entwicklungsbedürfnisse auf, die etwa im Prozess der Hochschulentwicklungsplanung hervortreten und bietet hierfür Dienstleistungen an, die in Kooperationsbeziehungen mit Fachbereichen eingebracht werden. Programme und Themen werden den Fachbereichen nicht von außen aufgesetzt, sondern kooperativ verabredet, gestaltet und eingebracht. Die Umsetzung von Gender Mainstreaming wird so weniger als top-down-Strategie wahrgenommen, denn als Lösungsansatz und Dienstleistung für die Bewältigung von Problemstellungen in den Fachbereichen. Damit ist viel gewonnen.

\section{Gender Mainstreaming als Querschnittsaufgabe in der Hochschulentwicklung: Gender-Portal}

Die Kooperation des Geschäftsbereichs mit Fachbereichen bei der Realisierung von Personalentwicklung für den wissenschaftlichen Nachwuchs ist eine wichtige Schiene der Umsetzung von Gender Mainstreaming. Darüber hinaus hat der Geschäftsbereich in Zusammenarbeit mit der Gleichstellungsbeauftragten eine Internet-Plattform „Gender-Portal“ für die Universität Duisburg-Essen eingerichtet, die als Arbeitsinstrument zur Wissensaneignung und Erweiterung der Gender-Kompetenz dienen soll.

Das Gender-Portal stellt Informationen und Materialien zur Umsetzung der politischen Gender Mainstreaming-Strategie sowie zur Gleichstellung und Frauenförderung bereit, ebenso aktuelle Ergebnisse der Frauen und Geschlechterforschung, die die Entwicklungsprozesse an der Universität Duisburg-Essen unterstützen können. Das Gender-Portal soll also nicht Gender-Wissen in aller Breite entfalten, wie andere entspre- 
chende internetbasierte Instrumente dies versuchen. Es werden vielmehr insbesondere die Themen aufgegriffen, die im Rahmen der Hochschulentwicklung der Universität Duisburg-Essen relevant sind; dies sind gegenwärtig:

- Gender in den Natur- und Ingenieurwissenschaften

- Geschlechtergerechte akademische Personalentwicklung

- Gender-Aspekte von Studiengangsentwicklung und -bewertung

Auf den Seiten des Gender-Portals wird auf die Entstehung, Bedeutung und Umsetzung von Gender Mainstreaming in den für die Hochschule zentralen Handlungsfeldern Bezug genommen: Personal- und Organisationsentwicklung, Studium und Lehre, Forschung sowie institutionalisierte Gleichstellungspolitik. Arbeitshilfen, Leitfäden und Checklisten sowie kommentierte weiterführende Links bündeln die Wissensbestände und unterstützen bei der konkreten Umsetzung von Gender Mainstreaming. Daneben sind auch Maßnahmen zur Frauenförderpolitik im Gender-Portal zu finden. So sind beispielsweise neben den verabschiedeten Frauenförderplänen der Fachbereiche auch Arbeitshilfen zur Erstellung von Frauenförderplänen abrufbar. Außerdem werden fachspezifische als auch fachübergreifende Programme der UDE zur Förderung des wissenschaftlichen (weiblichen) Nachwuchses vorgestellt.

Ein weiteres bedeutendes Handlungsfeld in der Hochschulentwicklung ist die Studienreform im Rahmen des Bologna-Prozesses. Gleichstellungsaspekte der Bologna-Reform betreffen insbesondere die Entwicklung, Akkreditierung und Umsetzung gestufter Studiengänge. Auch hierzu bietet das Gender-Portal ausgewählte Informationen.

Die Inhalte eines weiteren Themenfeldes, Gender \& Lehre, werden im Zusammenhang mit E-Learning sowie in Bezug auf die Präsenzlehre an der Hochschule erörtert. Außerdem werden Lehrveranstaltungen an der Uni Duisburg-Essen, die Gender thematisieren, veröffentlicht. Mit Hilfe dieses Gender-Vorlesungsverzeichnisses sind zum einen konkrete Beispiele für die Integration von Gender in die Lehre erhältlich, zum anderen wird interessierten Studierenden der Zugang zu Lehrveranstaltungen im Themenfeld Gender erleichtert.

Einen weiteren Schwerpunkt stellt das Thema Naturwissenschaften und Technik dar, da eine Erhöhung des Frauenanteils in diesen Fächern angestrebt wird. Um das Interesse für diesen zukunftsträchtigen Bereich nachhaltig zu erhöhen gibt es bereits zahlreiche Aktivitäten. Good Practice-Beispiele sind auf den Seiten des Gender-Portals ebenso zu finden wie Berufsverbände und Arbeitsgruppen für Frauen dieser Disziplinen sowie Kontaktdaten einschlägiger Einrichtungen. Fachspezifische Daten 
zur Studien- und Erwerbssituation von Frauen und Männern können ebenfalls abgerufen werden.

Im Gender-Portal werden außerdem Einrichtungen, Zeitschriften und Publikationen der Frauen- und Geschlechterforschung, vor allem des deutschsprachigen Raums, vorgestellt. Eine kommentierte Linksammlung bietet weiterführende Informationen. Außerdem ist ein Überblick über die Professuren an der UDE, die Frauen- und Geschlechterforschung thematisieren, erhältlich.

Neben Texten, Arbeitshilfen, Beispielprojekten und annotierten weiterführenden Links stellen die Seiten jeweils kommentierte Publikationen zur Verfügung. Service- und Beratungsangebote rund um das Thema Gender und Chancengleichheit, speziell an der UDE, sind ebenfalls im Gender-Portal zu finden. Zusätzlich zu den allgemeinen Daten und Fakten zur Studien- und Berufssituation von Frauen und Männern können auch die quantitativen Geschlechterverhältnisse der Studierenden, des wissenschaftlichen Nachwuchses und der Beschäftigten der UDE abgerufen werden. Darüber hinaus gibt es einen Veranstaltungskalender, der Tagungen und Konferenzen im deutschsprachigen Raum, aber auch Vorträge und Workshops an den Standorten Duisburg und Essen ankündigt.

Neben der Ausdifferenzierung der vorgestellten Themenfelder wird für die Zukunft der Ausbau des Portals auch als „Publikationsplattform“ geplant. Die Nutzung des Gender-Portals als interaktive Plattform steht ebenfalls noch am Anfang.

\section{Fazit}

Die Universität Duisburg-Essen hat zur Umsetzung ihrer GenderStrategie mit dem Ziel, Fortschritte in der Gleichstellung von Frauen und Männern zu erreichen, den Weg der Schaffung einer eigenen Einrichtung beschritten, die in diesem Aufgabenfeld Dienstleistungen für die Universität erbringen soll. Dieses Vorgehen scheint unter mehreren Aspekten erfolgsträchtig zu sein:

- Die Institutionalisierung der vormals nur temporär etablierten Projekte und Programme im Bereich von Frauenförderung und Gender Mainstreaming in einem Geschäftsbereich des Zentrums für Hochschul- und Qualitätsentwicklung bedeutet Verstetigung, auch im Sinne einer Ressourcensicherheit, die bei hinreichender Beweglichkeit auch langfristige strategische Entwicklungen voranbringt. 
- Die Struktur des Zentrums sichert, dass in allen strategischen Entwicklungsfeldern, auf die die Geschäftsbereiche ausgerichtet sind, Gender-Aspekte zur Geltung kommen.

- Gender Mainstreaming als top-down-Strategie wird vermittelt über Dienstleistungen; im Zusammenwirken von Management- und Steuerungsinstrumenten, insbesondere über den Zielvereinbarungsprozess, indem Ziele, aber auch Ressourcenbedarfe festgelegt werden, mit der Möglichkeit, entsprechende Dienstleistungen als Ressource abzurufen, werden Frauenförderung und Gender Mainstreaming in die Prozesse der Hochschul- und Organisationsentwicklung auch auf der Ebene der Fachbereiche eingebunden.

- Ausdrücklich ist der Charakter der Einrichtung als wissenschaftliche Dienstleistungseinrichtung zu betonen; er beinhaltet die Aufgabe, Dienstleistungen nicht standardisiert auf einem einmal gegebenen wissenschaftlichen Erkenntnisstand zu erbringen, sondern Dienstleistungen immer wieder unter wissenschaftlichen Gesichtspunkten zu vertiefen oder zu erneuern. Dies erlaubt Projekte und Programme in eigener Zuständigkeit zu entwickeln bei gleichzeitiger Offenheit für Dienstleistungsanforderungen.

- Die an der Universität Duisburg-Essen gewählte Institutionalisierungsform stellt Frauenförderung und Gender Mainstreaming in einen Zusammenhang, d.h. beide Ansätze werden als wechselseitig sich ergänzend betrachtet und in der gemeinsamen Zielperspektive ihres möglichen Beitrags zur Hochschulentwicklung verfolgt. Dies ermöglicht Synergien, z.B. in der Weise, dass Frauenförderprojekte zur Vermittlung von Gender-Kompetenz genutzt werden.

\section{Literatur}

Blickshäuser, Angelika/Bargen von, Henning (2006): Mehr Qualität durch Gender-Kompetenz, hg.v. der Heinrich-Böll-Stiftung, Königstein/Taunus.

BMFSFJ (2003): Gender Mainstreaming - Was ist das?, http://www. bmfsfj.de/Kategorien/Publikationen/Publikationen,did=5376.html.

Klees-Möller, Renate (2006): Meduse - von der Gründung eines Mentorinnennetzwerkes zur Implementierung des Geschäftsbereichs Frauenförderung/ Gender Mainstreaming in das Zentrum für Hochschulund Qualitätsentwicklung an der Universität Duisburg-Essen. In: Mentoring als Wettbewerbsfaktor an Hochschulen - strukturelle Ansätze der Implementierung, hg.v. Astrid Franzke/Helga Gotzmann, Münster. 
Klees-Möller, Renate/Petersen, Renate/Schönborn, Anette (2007): Gender Mainstreaming-Strategie in der Hochschul- und Qualitätsentwicklung an der Universität Duisburg-Essen - Das Beispiel Meduse. Dokumentation des AHD-Kongresses, (im Druck).

Löther, Andrea (2003): Mentoring-Programme für Frauen in der Wissenschaft. In: CEWS-Beiträge Frauen in Wissenschaft und Forschung. Nr. 1, hg.v. Brigitte Mühlenbruch, Bielefeld.

Rompeltien, Bärbel (2004): Mentoring für Hochschulabsolventinnen an der Schnittstelle Studium - Beruf: Flankierende Personalentwicklung. In: Flankierende Personalentwicklung durch Mentoring, hg.v. Sibylle Peters/Sonja Schmicker/Sibylle Weinert, München/Mering, S.87-99.

Universität Duisburg-Essen: Entwicklungsplanung, http://www.uniduisburg-essen.de/leitung/interne_entwicklungsplanung.shtml.

Zechlin, Lothar (2003): Kursbuch Strategische Entwicklung, Graz, http://www.uni-due.de/leitung/personen/zechlin.shtml. 


\section{Das Studienfach Gender Studies: ein Erfahrungsbericht über die Einrichtung des interdisziplinären Faches im Bundesland Bayern}

CORINNA ONNEN-ISEMANN

Ziel des vorliegenden Beitrags ist es, die Implementierung des Lehrgebietes Gender Studies an der Universität Regensburg zu beschreiben. Die Universität unterhält im Rahmen eines modularisierten Magisterstudienganges seit dem WS 2000/01 auch die Studieneinheit Gender Studies. Um der Bedeutung dieses Lehrgebiets auch strukturell einen Rahmen zu geben, konnten durch Initiative der Frauenbeauftragten im Sommer 2002 mit Unterstützung des Hochschulsonderprogramms die finanziellen Mittel bereitgestellt werden, den wissenschaftlichen Teil der Gender Studies durch eine Professur zu ermöglichen. Diese Professur wurde mit einer Sozialwissenschaftlerin besetzt und unterstrich so die interdisziplinäre Ausrichtung, von der hier berichtet wird.

\section{Die Ausgangslage}

Seit dem WS 2000/01 können an der Universität Regensburg als einziger Hochschule in Bayern Gender Studies im modularisierten MagisterStudiengang an den philosophischen Fakultäten als so genanntes „Frei kombinierbares Nebenfach" studiert werden.

Die Studieneinheit Gender Studies wurde im Rahmen des universitären Projektes „Leistungspunkte“ im Sommersemester 2000 eingerichtet (vgl. Studienreform konkret 2004). Zu der Zeit gab es bereits Lehr- und Lerninhalte der unterschiedlichen Disziplinen der vier philosophischen 
Fakultäten. Die Umstellung der bisherigen Diplom- und Magisterstudiengänge auf gestufte Studiengänge im Rahmen der Vorgaben des Bologna-Prozesses ermöglichte relativ flexible Wege zur Einrichtung von Modulen. $\mathrm{Zu}$ der Zeit wurden die Module anhand der bestehenden und bis dato durchgeführten Lehrveranstaltungen ,bestückt“: Modul 01 beinhaltete Vorlesungen, Seminare und Übungen aus den Bereichen Sprache, Literatur und Kunst und Modul 02 aus dem Lehrangebot in Geschichte, Gesellschaft, Theologie, Recht. Das Fach hatte zwar studentischen Zuspruch, aber die Betreuung und die Organisation der Studieneinheit waren nicht an eine Professur gebunden.

Nach umfangreichen Vorarbeiten konnten im Jahr 2002 die damaligen Frauenbeauftragten der Universität Regensburg durchsetzen, Mittel des Hochschulsonderprogramms zur Förderung von Frauen von der Bayerischen Staatskanzlei durch die Universität für die Errichtung einer Professur für Gender Studies zu beantragen.

Die Ausschreibung auf eine C3-Professur erfolgte als „Professur für Gender Studies“ (ohne weitere Fachbindung) im Frühjahr 2002 mit einer zeitlichen Befristung von zwei Jahren, die Berufungsvorträge waren am 26.6.02. Anfang November erging der Ruf durch das Bayerische Wissenschaftsministerium zur Besetzung der Stelle ab April 2003. Die Ausstattung der Stelle betrug drei Arbeitsplätze mit PC, Mittel für drei studentische Hilfskräfte, von denen eine Sekretariatstätigkeiten übernahm, sowie einen Grundbetrag an Bibliotheksmitteln; besetzt wurde die Professur mit der Diplom-Sozialwissenschaftlerin PD Dr. rer. pol. Corinna Onnen-Isemann.

\section{Die Professur für Gender Studies}

Bereits während der Aussprache im Rahmen des Berufungsverfahrens wurde deutlich, dass die Hochschule keine spezifischen Vorstellungen bezüglich der Ausgestaltung der Stelle hatte sondern vielmehr der neuen Stelleninhaberin einen möglichst großen Gestaltungsspielraum einräumen wollte. Lediglich die im Ausschreibungstext formulierten Anforderungen sollten erfüllt werden, nämlich:

- das Gebiet der Gender Studies in Lehre und empirischer Forschung $\mathrm{zu}$ vertreten,

- ein interdisziplinäres Lehrangebot für die beteiligten Fakultäten zu erstellen, sowie

- in einer geplanten interdisziplinären Forschungsgruppe Gender Studies mitzuwirken. 
Aufgrund dieser Bedingungen wurden in einem ersten Schritt die Studiengänge mit vergleichbarer Angebotsstruktur an deutschen Universitäten ermittelt und evaluiert. Anschließend wurde gemeinsam mit der für die Verwaltung der Studieneinheit zuständigen „Koordinierungsstelle Leistungspunkte“ und nach Gesprächen mit den Dekanen der Philosophischen Fakultät II und IV die Inhalte der Module stärker an ein Gender Studium angepasst.

Mit Wirkung vom Wintersemester 2003/04 galten schließlich die neuen nachfolgend dargestellten Studienmodule. Das Ziel dieser veränderten Modul-Struktur war es, einen Grundkanon von allgemeinen genderbasierten Inhalten dem Studium verpflichtend zuzuordnen, auf deren Basis dann die fachspezifischen Gender-Inhalte gelehrt werden konnten.

\section{Die Studieneinheit Gender Studies}

\section{Ziele}

Ziele der Studieneinheit Gender Studies sind:

- unter Berücksichtigung der Kategorie Geschlecht zu einem erweiterten Verständnis von Natur und Gesellschaft zu gelangen,

- zu vermitteln, wie Geschlechterverhältnisse in soziale und psychische Strukturen eingeschrieben sind und Auswirkungen auf das individuelle und strukturelle Handeln haben,

- Geschlechterverhältnisse als Machtverhältnisse zu analysieren.

Das Besondere der Studieneinheit besteht in ihrem interdisziplinären, d.h. die Fächergrenzen überschreitenden Charakter. Ein umfangreiches Lehrangebot aus den Kultur- und Sozialwissenschaften, den Sprach- und Literaturwissenschaften, der Pädagogik sowie den Naturwissenschaften ermöglicht es, Problembereiche aus der Sichtweise unterschiedlicher Fachgebiete zu betrachten. Hierzu wurden gemeinsam mit der Professurinhaberin durch intensive Gespräche Themen eruiert. Die Konzeption der Lehrveranstaltung oblag dann der Ausgestaltung der Fachdozenten bzw. Fachdozentinnen. Häufig trat durch diese Gespräche eine Gender Kompetenz zu Tage, die aufgrund der universitären Strukturen jedoch bislang nicht oder nur selten hervorkam, genauso häufig aber betraten Kollegen und Kolleginnen auch „Neuland“ - d.h. sie wagten sich mit der Lehre an für sie neue Gebiete heran und engagierten sich dann dafür umso mehr für diese „Gender-Lehre“. Dadurch bietet die Studieneinheit ein breites Spektrum von Perspektiven auf gesellschaftlich wichtige 
Themengebiete, die durch Bezugnahme auf die Kategorie „Geschlecht“ und auf Geschlechterverhältnisse neue Erkenntnisse eröffnen.

\section{Organisation und Gliederung der Studieneinheit}

Die Organisation der Studieneinheit Gender Studies erfolgt in der Philosophischen Fakultät II der Universität in enger Kooperation mit den beteiligten Fakultäten, insbesondere der Philosophischen Fakultät IV.

\section{Gliederung der Studieneinheit (vgl. Abb. 1-3)}

Die Studieneinheit Gender Studies gliedert sich in ein Grundstudium (1.-4. Semester) und in ein viersemestriges Hauptstudium. Beide Studienabschnitte sind modularisiert, mit einem Leistungspunktsystem versehen und werden durch studienbegleitende Prüfungen abgeschlossen.

Die Studienabschnitte sind in zwei Module gegliedert. Im Basismodul wird Überblickswissen über Theorien und Anwendungsmöglichkeiten der Gender-Forschung in verschiedenen Disziplinen gelehrt sowie das interdisziplinäre Denken und das fächerübergreifende Studium vermittelt. Das anschließende Aufbaumodul umfasst die Einführung in die Entwicklung von Geschlechterstudien, Theorien der Gender Studies, Geschlechterverhältnisse und geschlechtliche Arbeitsteilung sowie in die Methodologien und Methoden der Geschlechterforschung, Auseinandersetzung mit den Methoden des Hauptfaches oder Nebenfaches unter geschlechterspezifischer Perspektive. Der Besuch einer Methodenveranstaltung zu Gender Studies erfolgt zum Übergang ins Aufbaumodul.

Wird die Zwischenprüfung im Frei Kombinierbaren Nebenfach abgelegt, ist hierfür der Erwerb von insgesamt mindestens 30 Leistungspunkten, darunter je ein erfolgreich absolviertes Modul aus zwei Studieneinheiten, nachzuweisen.

Voraussetzungen: Weil Gender Studies im Rahmen des Frei Kombinierbaren Nebenfachs im Magisterstudiengang studiert werden, gelten die Rahmenbedingungen der Zwischenprüfungsordnung und der Magisterprüfungsordnung. Sprachkenntnisse in einer Fremdsprache werden vorausgesetzt.

Umfang der Ausbildung: Eine Studieneinheit entspricht quantitativ einem „halben“ Nebenfach. Anders ausgedrückt, die Studieneinheit Gender Studies umfasst mindestens 30 Leistungspunkte, das entspricht 5 Lehrveranstaltungen à 2 SWS (= 35 LP), die gleichmäßig im Grund- 
und Hauptstudium erbracht werden müssen. Die Inhalte der Veranstaltungen werden vom jeweiligen Fachgebiet bestimmt und konzipiert und wechseln daher von einem Semester zum anderen. Nach erfolgreich abgelegter Zwischenprüfung ist der Besuch von Hauptseminaren möglich. Im Grundstudium ist eine Lehrveranstaltung zum Thema „Einführung in die Gender Studies“ zu besuchen und zusätzlich sind Kenntnisse in empirischer Sozialforschung zu erwerben.

Die Einführungsveranstaltung wird curricular ein Mal jährlich jeweils im Wintersemester angeboten, die Methodenveranstaltung jeweils im Sommersemester.

Leistungsnachweise: Der Erwerb von Leistungsnachweisen findet in der üblichen Form durch Referate, Hausarbeiten und mündliche Prüfungen statt und wird durch die Magisterprüfungsordnung geregelt.

Mögliche Fächerkombinationen: Im Magisterstudiengang kann die Studieneinheit Gender Studies im Rahmen des Frei Kombinierbaren Nebenfachs mit allen Magisterfächern kombiniert werden. 
Tab. 1: Studieneinheit Gender Studies: Modulstruktur GEN ${ }^{1}$

\begin{tabular}{|c|c|}
\hline 1. Studieneinheit: & Gender Studies \\
\hline 2. Fachgebiet/Verantwortlich: & $\begin{array}{l}\text { Gender Studies/Prof. Dr. Corinna } \\
\text { Onnen-Isemann }\end{array}$ \\
\hline 3. Inhalte/Lehrziele: & $\begin{array}{l}\text { Überblick über Theorie und An- } \\
\text { wendungsmöglichkeiten der Gen- } \\
\text { der-Forschung in verschiedenen } \\
\text { Disziplinen, Förderung des inter- } \\
\text { disziplinären Denkens und des fä- } \\
\text { cherübergreifenden Studiums. }\end{array}$ \\
\hline $\begin{array}{l}\text { 4. Voraussetzungen: } \\
\text { a) allgemeiner Art: } \\
\text { b) vorausgesetzte universitäre } \\
\text { Lehrveranstaltungen }\end{array}$ & $\begin{array}{l}\text { a) Gesicherte Englischkenntnisse } \\
\text { b) ----- }\end{array}$ \\
\hline 5. Bedingungen: & ----- \\
\hline 6. Modularer Aufbau: & $\begin{array}{l}\text { Pflicht im Grundstudium ist das } \\
\text { Basismodul. Bis zum Ende des } \\
\text { Hauptstudiums muss das Aufbau- } \\
\text { modul abgeschlossen sein. }\end{array}$ \\
\hline $\begin{array}{c}\text { GEN - M } 01 \text { Basismodul } \\
\text { Gender Studies }\end{array}$ & $\begin{array}{c}\text { GEN - M } 10 \text { Aufbaumodul } \\
\text { Gender Studies } \\
\end{array}$ \\
\hline $\begin{array}{l}\text { 7. Kursangebot/zeitlicher Rah- } \\
\text { men: }\end{array}$ & $\begin{array}{l}\text { Ein Modul kann in zwei Semestern } \\
\text { absolviert werden. Die Inhalte der } \\
\text { Lehrveranstaltungen werden von } \\
\text { Gender Studies und dem jeweili- } \\
\text { gen Fachgebiet bestimmt und } \\
\text { wechseln deshalb von einem Se- } \\
\text { mester zum anderen. }\end{array}$ \\
\hline
\end{tabular}

Quelle: Eigene Darstellung

1 Die Abbildungen 1-3 wurden zusammengestellt aus http://www.uniregeburg.de/Fakultaeten/phil_Fak_IV/Modularisierung/magist2/downloadFKN /genneu.doc 
Tab. 2: Studieneinheit Gender Studies: Modulbeschreibung GEN M01 ${ }^{1}$

\begin{tabular}{|c|c|}
\hline 1. Name des Moduls: & Basismodul Gender Studies \\
\hline 2. Fachgebiet & Gender Studies \\
\hline 3. Inhalte/Lehrziele & $\begin{array}{l}\text { Einführung in die Entwicklung } \\
\text { von Geschlechterstudien, } \\
\text { Theorien der Gender Studies, } \\
\text { Geschlechterverhältnisse und } \\
\text { geschlechtliche Arbeitsteilung } \\
\text { sowie in die Methodologien } \\
\text { und Methoden der } \\
\text { Geschlechterforschung, } \\
\text { Auseinandersetzung mit den } \\
\text { Methoden des Hauptfaches oder } \\
\text { Nebenfaches unter } \\
\text { geschlechterspezifischer } \\
\text { Perspektive }\end{array}$ \\
\hline $\begin{array}{l}\text { 4. 4. Voraussetzungen: } \\
\text { a) allgemeiner Art: } \\
\text { b) vorausgesetzte universitäre } \\
\text { Lehrveranstaltungen } \\
\end{array}$ & $\begin{array}{l}\text { a) Gesicherte } \\
\text { Englischkenntnisse } \\
\text { b) keine }\end{array}$ \\
\hline \multicolumn{2}{|l|}{ 5. Bedingungen: } \\
\hline - verwendbar in: & Studieneinheit Gender Studies \\
\hline \multicolumn{2}{|l|}{$\begin{array}{l}\text { - nicht verwendbar in/nicht } \\
\text { kombinierbar mit: }\end{array}$} \\
\hline $\begin{array}{l}\text { 6. Wie häufig wird das Modul } \\
\text { angeboten? }\end{array}$ & $\begin{array}{l}\text { Einmal pro Studienjahr, Veran- } \\
\text { staltung Nr.1 wird jedes WS } \\
\text { angeboten, Veranstaltung Nr. } 2 \\
\text { jedes SS }\end{array}$ \\
\hline $\begin{array}{l}\text { 7. In welcher Zeit kann das Modul } \\
\text { absolviert werden? }\end{array}$ & In zwei Semestern \\
\hline 8. Zusammensetzung: & $\begin{array}{l}\text { Vor dem Besuch der } \\
\text { Veranstaltung Nr. } 2 \text { muss die } \\
\text { Veranstaltung Nr. } 1 \text { erfolgreich } \\
\text { absolviert sein }\end{array}$ \\
\hline 9. & $\begin{array}{l}\text { Die Veranstaltungen sind im } \\
\text { Rahmen der für die Prüfungen } \\
\text { gesetzten Fristen wiederholbar. }\end{array}$ \\
\hline 10. & $\begin{array}{l}\text { Die Endnote des Moduls wird } \\
\text { aus dem Durchschnitt der in } \\
\text { den Veranstaltungen Nr. 1-3 } \\
\text { erreichten Noten ermittelt. }\end{array}$ \\
\hline
\end{tabular}




\begin{tabular}{|l|l|l|l|}
\hline Nr. & Veranstaltungen & SWS & LP \\
\hline & A Pflichtbereich & & \\
\hline 1 & Einführung in Gender Studies & 2 & 5 \\
\hline 2 & Einführung in die Methoden der Gender Studies & 2 & 5 \\
\hline 3 & $\begin{array}{l}\text { Vorlesung, Übung oder Seminar aus Sprachwissen- } \\
\text { schaft oder Vorlesung, Übung oder Seminar aus Lite- } \\
\text { raturwissenschaft oder Vorlesung, Übung oder Semi- } \\
\text { nar aus Kunstwissenschaft oder Vorlesung, Übung } \\
\text { oder Seminar aus Gesellschaftswissenschaft oder } \\
\text { Vorlesung, Übung oder Seminar aus Geschichtswis- } \\
\text { senschaft oder Vorlesung, Übung oder Seminar aus } \\
\text { Pädagogik oder Vorlesung, Übung oder Seminar aus } \\
\text { Psychologie oder Vorlesung, Übung oder Seminar aus } \\
\text { Theologie oder Vorlesung, Übung oder Seminar aus } \\
\text { Rechtswissenschaft oder Vorlesung, Übung oder Se- } \\
\text { minar aus Wirtschaftswissenschaft oder }\end{array}$ & 7 & 2 \\
\hline $\begin{array}{l}\text { B Wahlbereich } \\
\text { Summe aus dem Pflichtbereich }\end{array}$ & 6 & 17 \\
\hline
\end{tabular}

Quelle: Eigene Darstellung

Tab. 3: Studieneinheit Gender Studies: Modulbeschreibung GEN M01 ${ }^{1}$

\begin{tabular}{|c|c|}
\hline 1. Name des Moduls: & Aufbaumodul Gender Studies \\
\hline 2. Fachgebiet & Gender Studies \\
\hline 3. Inhalte/Lehrziele: & $\begin{array}{l}\text { Problemfelder und } \\
\text { Schwerpunkte der Gender } \\
\text { Studies aus einer Disziplin im } \\
\text { Hauptfach bzw. Nebenfach }\end{array}$ \\
\hline \multicolumn{2}{|l|}{ 4. Voraussetzungen: } \\
\hline a) allgemeiner Art & $\begin{array}{l}\text { Gesicherte } \\
\text { Englischkenntnisse }\end{array}$ \\
\hline $\begin{array}{l}\text { b) vorausgesetzte universitäre } \\
\text { Veranstaltungen: }\end{array}$ & $\begin{array}{l}\text { Abgeschlossenes Basismodul } \\
\text { Gender Studies }\end{array}$ \\
\hline \multicolumn{2}{|l|}{ 5. Bedingungen: } \\
\hline - verwendbar in: & Studieneinheit Gender Studies \\
\hline \multicolumn{2}{|l|}{$\begin{array}{l}\text { - nicht verwendbar in/nicht } \\
\text { kombinierbar mit: }\end{array}$} \\
\hline $\begin{array}{l}\text { 6. Wie häufig wird das Modul } \\
\text { angeboten? }\end{array}$ & Jedes Semester \\
\hline
\end{tabular}




\begin{tabular}{|c|c|c|c|c|}
\hline \multicolumn{2}{|c|}{$\begin{array}{l}\text { 7. In welcher Zeit kann das Modul } \\
\text { absolviert werden? }\end{array}$} & \multicolumn{3}{|c|}{ In maximal zwei Semestern } \\
\hline \multicolumn{2}{|c|}{ 8. Zusammensetzung: } & \multicolumn{3}{|c|}{$\begin{array}{l}\text { Veranstaltungen aus zwei der } \\
\text { angebotenen Bereiche }\end{array}$} \\
\hline \multicolumn{2}{|l|}{9.} & \multicolumn{3}{|c|}{$\begin{array}{l}\text { Die Veranstaltungen sind im } \\
\text { Rahmen der für die Prüfungen } \\
\text { gesetzten Fristen wiederhol- } \\
\text { bar }\end{array}$} \\
\hline \multicolumn{2}{|l|}{10.} & \multicolumn{3}{|c|}{$\begin{array}{l}\text { Die Endnote des Moduls wird } \\
\text { aus der in den beiden Veran- } \\
\text { staltungen erreichten Noten } \\
\text { ermittelt. }\end{array}$} \\
\hline \multirow[t]{2}{*}{ Nr. } & \multicolumn{2}{|l|}{ Veranstaltungen } & SWS & LP \\
\hline & \multicolumn{2}{|l|}{ A Pflichtbereich } & & \\
\hline 1 & \multicolumn{2}{|c|}{$\begin{array}{l}\text { Vorlesung, Übung oder Seminar aus Sprachwis- } \\
\text { senschaft }\end{array}$} & 2 & 7 \\
\hline 2 & \multicolumn{2}{|c|}{$\begin{array}{l}\text { Vorlesung, Übung oder Seminar aus Literaturwis- } \\
\text { senschaft }\end{array}$} & 2 & 7 \\
\hline 3 & \multicolumn{2}{|c|}{$\begin{array}{l}\text { Vorlesung, Übung oder Seminar aus Kunstwissen- } \\
\text { schaft }\end{array}$} & 2 & 7 \\
\hline 4 & \multicolumn{2}{|c|}{$\begin{array}{l}\text { Vorlesung, Übung oder Seminar aus Gesell- } \\
\text { schaftswissenschaft }\end{array}$} & 2 & 7 \\
\hline 5 & \multicolumn{2}{|c|}{$\begin{array}{l}\text { Vorlesung, Übung oder Seminar aus Geschichts- } \\
\text { wissenschaft }\end{array}$} & 2 & 7 \\
\hline 6 & \multicolumn{2}{|c|}{ Vorlesung, Übung oder Seminar aus Pädagogik } & 2 & 7 \\
\hline 7 & \multicolumn{2}{|c|}{ Vorlesung, Übung oder Seminar aus Psychologie } & 2 & 7 \\
\hline 8 & \multicolumn{2}{|c|}{ Vorlesung, Übung oder Seminar aus Theologie } & 2 & 7 \\
\hline 9 & \multicolumn{2}{|c|}{$\begin{array}{l}\text { Vorlesung, Übung oder Seminar aus Rechtswis- } \\
\text { senschaft }\end{array}$} & 2 & 7 \\
\hline \multirow[t]{2}{*}{10} & \multicolumn{2}{|c|}{$\begin{array}{l}\text { Vorlesung, Übung oder Seminar aus Wirtschafts- } \\
\text { wissenschaft }\end{array}$} & 2 & 7 \\
\hline & \multicolumn{2}{|l|}{$\begin{array}{l}\text { B Wahlbereich } \\
\text { Summe aus dem Pflichtb }\end{array}$} & 2 & 14 \\
\hline
\end{tabular}

Quelle. Eigene Darstellung 


\section{Entwicklung der Studierendenzahlen}

Im Sommersemester 2003 betrug die Zahl der eingeschriebenen Studierenden 22, bei der letzen Erhebung durch die Koordinierungsstelle Leistungspunkte zu Beginn des Wintersemesters 2004/05 beläuft sie sich auf 72. Die Entwicklung der Studierendenzahlen in den frei kombinierbaren Nebenfächern zeigt die folgende Grafik:

Abb. 1: Studierende der fünf meist gewählten frei kombinierbaren Nebenfächer (FKN) seit Sommersemester 2003

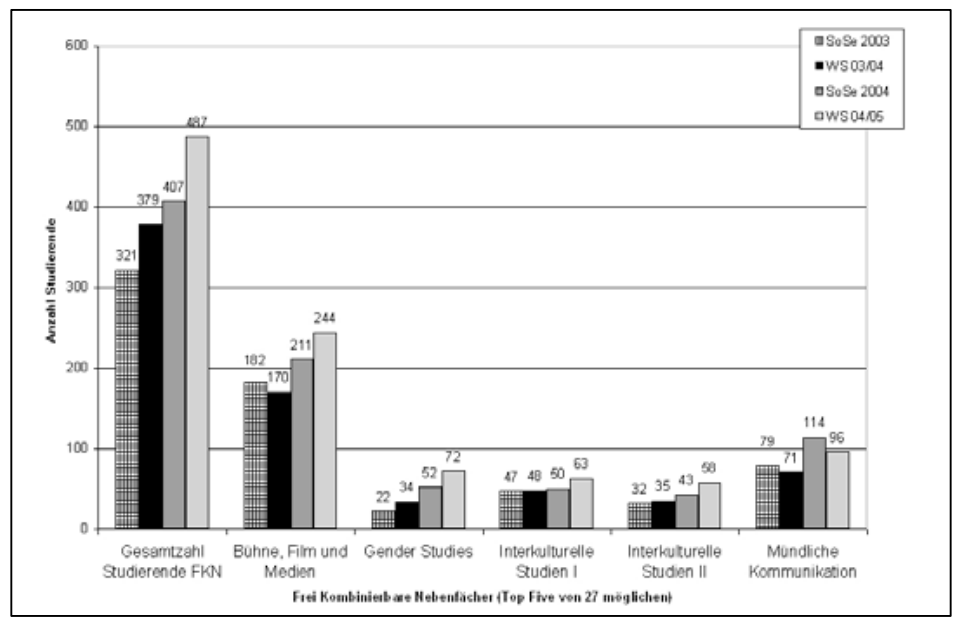

Quelle: Studienjahrgangserhebungen http://www.uni-reburg.de/Fakultaeten/phil_Fak_IV/Modularisierung/magist2/struktur.

htm, eigene Berechnungen 


\section{Evaluation der Lehrveranstaltungen in Gender Studies}

Abb. 2: Belegung der Studieneinheiten

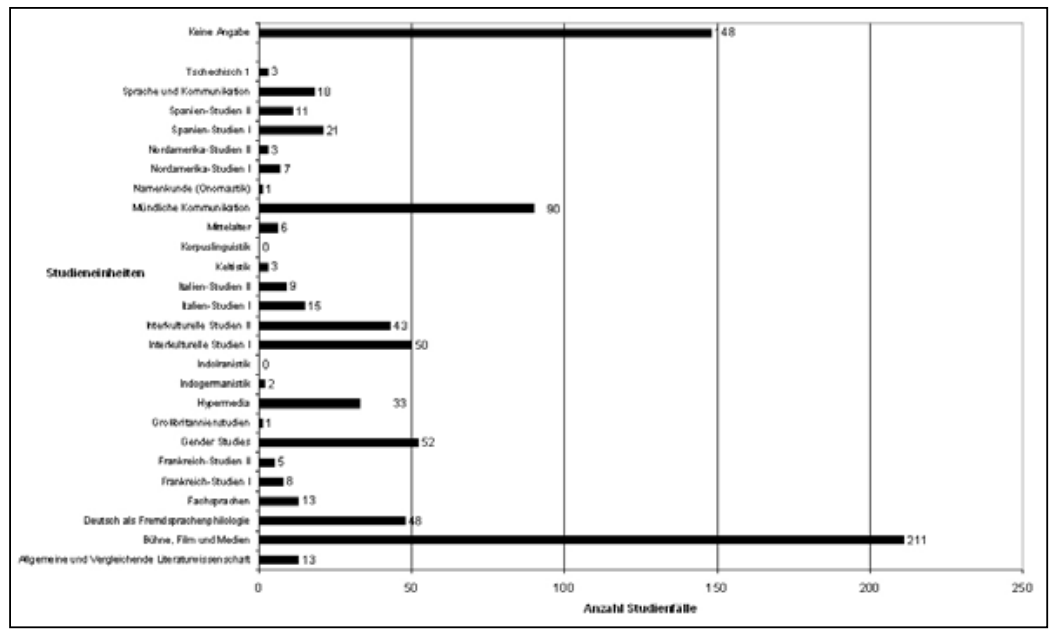

Quelle: Studienjahrgangserhebungen http://www.uni-regensburg.de/ Fakultaeten/phil_Fak_IV/Modularisierung/magist2/struktur. Htm

Abb. 3: Prognose der Studienfachbelegung

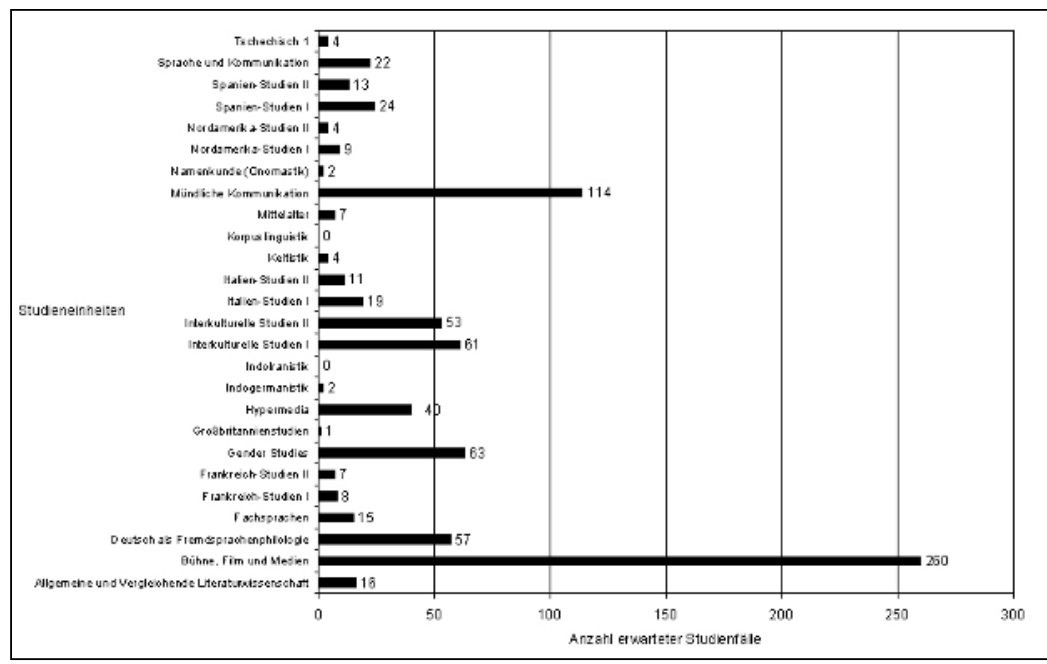

Quelle: Studienjahrgangserhebungen http://www.uni-regensburg.de/ Fakultaeten/phil_Fak_IV/Modularisierung/magist2/struktur.htm, eigene Berechnungen 
Abb. 4: Prozentuale Verteilung der Studierenden auf die FKN

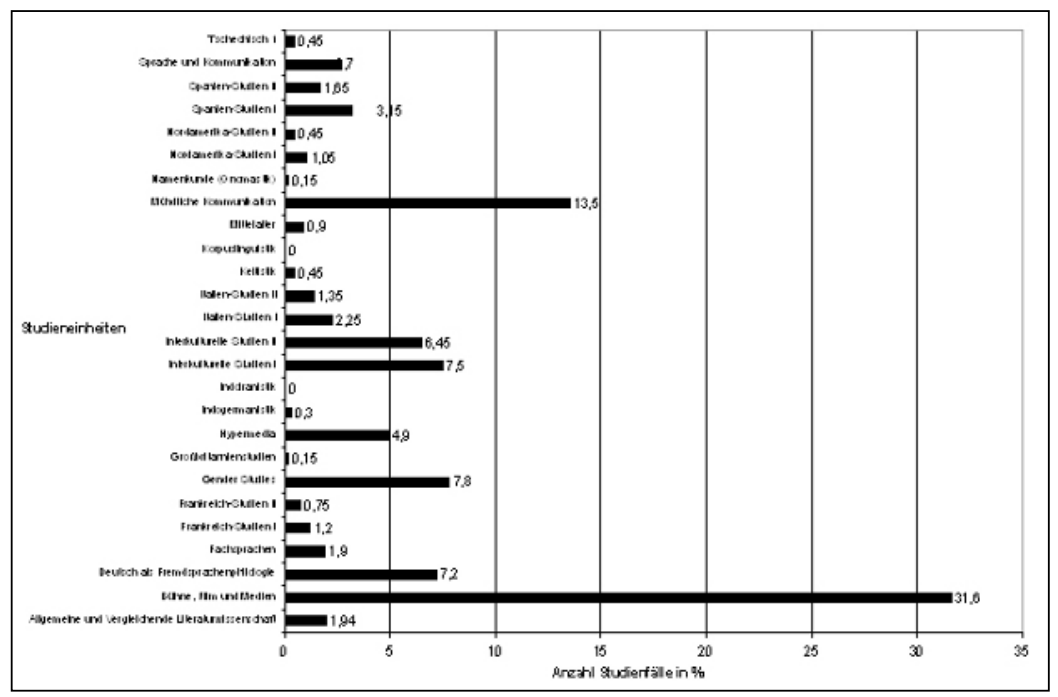

Quelle: Studienjahrgangserhebungen http://www.uni-regensburg.de/

Fakultaeten/phil_Fak_IV/Modularisierung/magist2/struktur.htm

\section{Gender Studies in der Forschung}

\section{Universitär}

Innerhalb der Universität Regensburg wurde ein Arbeitskreis Gender Studies gegründet, der sich mehrfach traf und Themen der fachspezifischen Forschung und der Lehre besprach. Die Professurinhaberin übernahm die Leitung und die damit verbundenen Verwaltungstätigkeiten (Einladungen, Festlegung der Tagesordnungspunkte etc.) und konnte so jeweils zwischen zehn und fünfzehn Mitglieder zusammenhalten Hieraus speiste sich dann die im darauffolgenden Semester stattfindende 14tägliche Ringvorlesung. Auf diese Weise konnte der interdisziplinäre Charakter verdeutlicht werden und aufgrund einer aktiven Pressearbeit genderspezifische Fragestellungen einem breiten öffentlichen Publikum zugänglich gemacht werden. Die einzelnen Vorlesungen speisten sich ausnahmslos durch Kollegen und Kolleginnen der Universität Regensburg und waren jede mit 60-80 Zuhörenden besetzt.

Eine wissenschaftliche interdisziplinäre Fachtagung zum Thema „Schwestern - Dynamik und Repräsentation einer lebenslangen Beziehung“ erreichte ebenfalls eine große Resonanz. Ferner konnte ein gemeinsames Projekt mit der Fachhochschule Regensburg im Rahmen ei- 
nes neu akkreditierten Weiterbildungsstudienganges (seit WS 2005/06) „Leitungs- und Kommunikationsmanagement“ realisiert werden, das ein Modul als „Gender Mainstreaming und Gender Studies“ zwingend voraussetzt. Dieser Studiengang finanziert sich durch Studiengebühren und rekrutiert hauptsächlich Personen mit Personalverantwortung.

Schließlich konnte ein Drittmittel-Projekt initiiert werden zur Entwicklung eines Gender Moduls im Rahmen des e-learnings an der Virtuellen Hochschule Bayern. Dieses Modul wird 2008 an den Start gehen.

\section{Außeruniversitär}

Angeregt durch dieses externe Interesse an den genderspezifischen Themen gründete die Stelleninhaberin gemeinsam mit örtlichen Funktionsträgern und Funktionsträgerinnen (der Gleichstellungsstelle der Stadt Regensburg, der Agentur für Arbeit und der Industrie- und Handelskammer) den Arbeitskreis ImPuls ${ }^{\circledR}$ Regensburg. Ziel war es, in unserer wissensbasierten Wirtschaft den Transfer von Wissen in die Unternehmen zu beschleunigen, und so Innovationen zu befördern. Dieser Arbeitskreis wurde ergänzt durch ein breites Klientel an Organisationsberaterinnen und -beratern, hohen Funktionsträgern und Funktionsträgerinnen des öffentlichen Dienstes, der Klein- und Mittelbetriebe sowie der örtlichen Großindustrie. Er hatte - neben der Aufgabe eines ,runden Tisches“ - zum Ziel, Gender Mainstreaming Konzepte in die jeweiligen Arbeitsbereiche hineinzutragen und gezielte fachliche Hilfen bei der Umsetzung zu erarbeiten.

Zwei Mal jährlich wurden größere Veranstaltungen zu GenderThemen durchgeführt, von denen eine Veranstaltung im Januar 2005 des Club „business and professional women“ (bpw) erhebliches politisches Interesse erreichte. Unter anderem auch aufgrund dieser Resonanz wies der bayerische Wissenschaftsminister Dr. Thomas Goppel im März 2005 der Universität Regensburg eine W2-Professur für Gender Studies zu. Da die Besetzung der Stelle lt. Hochschulrecht bei einer Neueinrichtung nach passieren der universitären Strukturkommission ausgeschrieben werden muss, erfolgte die Neuausschreibung Anfang 2006 als „Professur für Public Health mit besonderem Schwerpunkt Gender Studies“ und ist somit in der Medizinischen Fakultät angesiedelt - der interdisziplinäre Charakter der Stelle dürfte damit vorüber sein.

Des Weiteren konnten direkte Kooperationen mit öffentlichen Trägern und Organisationen angebahnt werden (z.B. in diversen Gremien der Stadt Regensburg, den Frauenhäusern der Stadt, Behörden im Kreis, der Polizei, der Finanzdirektion, einzelnen Schulbezirken sowie spezifischen Schulen in der Oberpfalz, den Volkshochschulen, der Caritas, der 
Diakonie, ProFamilia und Donum Vitae, dem Bischöflichen Ordinariat). Auch Gender Projekte mit Industriebetrieben wurden und werden durchgeführt und z.B. bestehende Diversity-Konzepte evaluiert.

\section{Fazit}

Universitätsintern strukturell begründet wurde die neu geschaffene Professur mit einer Soziologin besetzt und innerhalb der Pädagogik formal verankert. Dieses gewährleistete zwar einerseits die für eine Professur notwendige formale Zugehörigkeit in universitäre Strukturen (Fakultät, Fachbereich, Institut) - eine inhaltliche Einbindung in fachspezifische Belange der Pädagogik war jedoch nicht möglich und auch von beiden Seiten nicht gefordert.

Die Gender Studies hatten somit die Möglichkeit, sich von vornherein als eigenständige Disziplin zu verorten. Um dieses zu beschleunigen, wurden auch universitätsintern während der gesamten Dauer der Professur genderspezifische wissenschaftliche Ansätze zu erklären versucht und nach und nach konnten immer mehr Kollegen und Kolleginnen der unterschiedlichen Disziplinen für eine Zusammenarbeit in GenderThemen gewonnen werden. Diese Zusammenarbeit funktionierte hauptsächlich im Rahmen des Arbeitskreises und im Rahmen der o.g. Ringvorlesung, wo sich ein Raum bot, aus unterschiedlichen Disziplinen über Gender Studies zu diskutieren.

Die zeitliche Befristung der Professur auf zwei Jahre erlaubte jedoch keine inhaltlich Aufwertung des Faches zum Beispiel als vollwertiges Nebenfach oder als Hauptfach, was laut Strukturordnung der Universität nur mit einer unbefristeten Professur möglich gewesen wäre. Somit lag es unter anderem an den Universitätsstrukturen, dass dem Fach die nötige institutionelle Unterstützung an der Universität Regensburg verwehrt blieb. Die überall im Hochschulsystem spürbaren Restriktionen bezüglich der verschiedenen finanziellen Ausstattungen der einzelnen Fächer zeigten sich insbesondere darin, dass an eine Ressourcenneu- bzw. -umverteilung angesichts der knappen Kassen nicht zu denken war. Vielmehr konnte beobachtet werden, dass eine Besitzstandswahrung der etablierten Fächer positiv korrelierte mit dem Anstieg der Sparmaßnahmen.

Das Fazit dieses Modellversuchs in Regensburg lässt sich knapp formulieren: der geringste Dissens herrschte bei der grundsätzlichen fachlichen inhaltlichen Anerkennung der Gender Studies, der größte jedoch sobald konkrete Unterstützung gefordert wurde. Ohne massive sowie eine institutionelle und strukturelle Verankerung dieses neuen Fa- 
ches ist eine Etablierung ,wie von selbst“ unmöglich. Nach Auslaufen der Professur war schnell der Status quo ante wieder hergestellt: jede und jeder Lehrende lehrt nun wieder frei und ohne inhaltliche Etablierung des Faches zu Gender-Themen - dieses Konstrukt hilft jedenfalls dem Fach an sich nicht weiter. Aufgrund dieser Erfahrungen sollte in Zukunft bei Versuchen, Gender Studies an einer Hochschule zu verankern zunächst ein deutlicher Schwerpunkt auf der institutionellen Verankerung gesetzt werden, incl. der dafür notwendigen Strukturänderungen in der universitären Grundordnung - ansonsten hat das Fach keine Chance sich zu etablieren.

\section{Literatur}

Studienreform konkret (2004): Handbuch zur Einführung eines Leistungspunktesystems an deutschen Hochschulen, Regensburg. 



\section{Gender und Innovationen - Erfahrungen aus dem Projekt „Discover Gender!“}

NinA BESSING UND Helga LuKOSCHAT

Der Artikel skizziert die Erfahrungen des Projekts „Discover Gender“, das die Fraunhofer Gesellschaft von 2003 bis 2006 in einem interdisziplinärem Team durchgeführt hat. Der Artikel geht dabei insbesondere auf die Bedeutung der Gender- und Innovationsforschung für die anwendungsorientierte Forschung und Technikentwicklung ein.

\section{Einleitung}

Inwieweit führt die Integration der Gender-Dimension zu einer erhöhten Innovationsfähigkeit in Forschung und Entwicklung? Welche Blickerweiterung und welche Chancen und Potenziale sind damit für die erhöhte Kundinnen- und Kundenzufriedenheit verbunden? Und: Wie kann den Prozessen der Benachteiligung und Stereotypisierung von Frauen, zum Beispiel in der Produktentwicklung, entgegen gewirkt werden?

Mit diesen Fragen startete das Projekt „Discover Gender“, das von der Fraunhofer Gesellschaft mit Förderung des Bundesministeriums für Bildung und Forschung von 2003 bis 2006 in einem interdisziplinären Projektteam $^{1}$ durchgeführt wurde. Übergreifendes Ziel des Projekts war

1 Die Projektleitung lag bei der Zentrale der Fraunhofer Gesellschaft. Projektpartner waren folgende Organisationen: Europäische Akademie für Frauen in Politik und Wirtschaft (EAF), Fraunhofer Institut System- und Innovationsforschung (ISI), Trommsdorff \& Drüner, Innovation und Marketing Consultans GmbH und Wehking PR. Das Projekt wurde mit Mitteln des Bundesministeriums für Bildung und Forschung gefördert. 
es, Wissensgrundlagen und Methodiken für die Integration von GenderAspekten in die anwendungsorientierte Forschung und Technologieentwicklung zu erarbeiten. Dabei kombinierte das Projekt im Wesentlichen drei Fragestellungen: Erstens ging es darum in ausgewählten Forschungsgebieten der Fraunhofer Gesellschaft Gender-Aspekte zu ermitteln, über konkrete Beispiele zu veranschaulichen und damit die Relevanz von Gender sichtbar zu machen.

Zweitens interessierte uns die Fragestellung wie, mit welchen Prozessen, Instrumenten und Methodiken, es möglich wird, Gender-Aspekte aufzudecken und in Forschungs- und Entwicklungs- (F\&E) Prozesse zu integrieren.

Drittens war es unser Ziel, Forscher und Forscherinnen in der Fraunhofer Gesellschaft zu sensibilisieren und Erfahrungen über geeignete Sensibilisierungsstrategien zu sammeln.

Diese drei Fragestellungen wurden verbunden mit einer innovationspolitischen Perspektive: Das deutsche Innovationssystem steht vor großen Herausforderungen, aber auch vor großen Chancen. Nach wie vor gehört Deutschland zu den im internationalen Vergleich innovativsten Volkswirtschaften. Neuere Studien weisen allerdings darauf hin, dass das deutsche Innovationssystem seine Leistungsfähigkeit erhöhen könnte, wenn es gelingen würde,

- die inhaltlichen Potenziale der Gender-Forschung für die Qualität und Bedarfsgerechtigkeit anwendungsorientierter Forschung und Technikentwicklung fruchtbar $\mathrm{zu}$ machen (vgl. Schraudner/Lukoschat 2006; Buhr 2006) und

- die Potenziale und Begabungen von Hochschulabsolventinnen und Forscherinnen für das Innovationsmanagement und für Forschungsund Entwicklungsprozesse in der Industrie besser als bisher zu erschließen (vgl. Deutsche Telekom Stiftung 2005; Europäische Kommission 2006).

Die innovationspolitische Bedeutung von Gender-Aspekten in der anwendungsorientierten Forschung und Technikentwicklung enthält also eine inhaltliche und eine personalpolitische Dimension.

Bei der inhaltlichen Dimension, wie wir sie im Projekt verstanden haben, geht es um die Integration der Erfahrungen und Theorien der Gender-Forschung in anwendungsorientierte Forschung- und Technikentwicklung und damit letztendlich um eine gendersensible Erweiterung des Blickfeldes. Die personalpolitische Dimension bezieht sich vor allem auf die Förderung und die Integration von Frauen in anwendungs- 
orientierte Forschung und Technikentwicklung und die damit verbundenen Maßnahmen zur Personal- und Organisationsentwicklung.

Die meisten Gender-Projekte bezogen sich in der Vergangenheit auf die personalpolitische Dimension, daher startet das Projekt „Discover Gender!“ ganz klar mit dem Ziel, die inhaltliche Dimension zu beleuchten.

Im vorliegenden Beitrag werden die Erfahrungen, die wir im Laufe des Projekts gemacht haben, dargelegt.

\section{Zum Grundverständnis von Gender}

Die Auseinandersetzung mit grundsätzlichen Fragen der GenderForschung begleitete sowohl uns als interdisziplinäres Projektteam als auch unsere Arbeit mit den Forscherinnen und Forschern der Fraunhofer Gesellschaft von Anfang an. Unser definiertes Ziel war es, durch den Einbezug der Gender-Dimension einen Beitrag zur Qualitätserhöhung anwendungsorientierter Forschung und Technikentwicklung zu leisten. Ziel war es auch, die gleiche Teilhabe von Frauen und Männern an der Entwicklung technischer Lösungen zu ermöglichen und damit letztendlich vielfältige und bedarfsgerechte Nutzungsoptionen von Technologien auch jenseits traditioneller Geschlechtsrollen zu ermöglichen.

Die theoretische wie praktische Herausforderung ist, GenderAspekte in anwendungsorientierter Forschung und Technikentwicklung zu berücksichtigen, ohne Stereotype und Rollenmuster zu reproduzieren. Unsere Erfahrung in dem Projekt hat gezeigt, dass dies vor allem bedeutet, den Begriff Gender und seine Bedeutung im jeweiligen Forschungskontext beständig zu reflektieren und Gender einzubetten in ein umfassenderes Konzept von Diversität. Je nach Forschungsfeld und Themenstellung können mehrere Differenzierungsmerkmale wie Nationalität, Alter oder berufliche Sozialisation relevant sein. So zeigte sich z.B. bei einem unserer „Nutzer/innen-Workshops“, der die Testung eines neu entwickelten Prototyps zur Bluthochdruckmessung zum Ziel hatte, dass die Anforderungen an das Gerät maßgeblich von Alter, Geschlecht und Beruf der Nutzerinnen und Nutzer bestimmt wurde. Geschlechtsunterschiede allein waren nicht in der Lage die unterschiedlichen Präferenzen zu erklären, doch ohne den Einbezug der Variable Geschlecht hätten wir z.B. nicht herausfinden können, dass die befragten Nutzerinnen bereit waren mehr Geld für das Bluthochdruckgerät auszugeben als die befragten Nutzer. Dabei gilt es, den Blick nicht nur auf individuelle Geschlechtsunterschiede, sondern auch auf die organisationale und gesellschaftliche Ebene zu lenken und den Zusammenhang zwischen diesen 
verschiedenen Ebenen zu berücksichtigen. In einem Workshop mit Forscherinnen und Forschern aus der Verkehrs- und Umweltforschung betrachteten wir beispielsweise die Ergebnisse der Forschung zu gesellschaftlichen Mobilitätsmustern und leiteten daraus Schlussfolgerungen für die konkreten Projekte der Forscherinnen und Forscher ab: Frauen sind immer noch stärker für Hausarbeit und Familienarbeit zuständig und legen daher im Durchschnitt pro Tag viele eher kürzere Wegstrecken zurück und gehen häufiger zu Fuß oder benutzen das Fahrrad. Männer sind häufig Vollzeit erwerbstätig und legen daher eher wenige und längere Wegstrecken zurück. Diese unterschiedlichen Mobilitätsmuster resultieren nicht aus einem generellen Unterschied zwischen Frauen und Männern, sondern aus gesellschaftlichen Anforderungen an Erwerbstätigkeit und Reproduktionsarbeit (Klima-Bündnis/Stete Planung 2001, zit.n. Genanet 2001:1). Beide Formen der Arbeit sind für die Gesellschaft notwendig und sollten daher bei der Entwicklung von nutzungsgerechten Verkehrssystemen berücksichtigt werden.

Der Einbezug des sozialen Kontextes lässt auch die Kopplung von Geschlechtszugehörigkeit mit Diskriminierungen auf gesellschaftlicher und/ oder organisationaler Ebene sichtbar werden. Diese Zusammenhänge den Forscherinnen und Forschern aufzuzeigen war ebenfalls wichtig. Die konstruktivistische Perspektive in der Geschlechterforschung macht deutlich, dass Geschlechtsunterschiede zum großen Teil auch ,gemachte Geschlechtsunterscheidungen“" sind (Krell 2004: 27), die als Legitimierung für Diskriminierungen dienen können. Wenn wir Gender-Aspekte in FuE-Projekten betrachten, reicht es also nicht aus, die Unterschiede zwischen Männern und Frauen zu berücksichtigen. Im Gegenteil: Gender-Aspekte können sich auch dort zeigen, wo bisher von Unterschieden, z.B. im Sinne von Rollenklischees, ausgegangen wird, obwohl vielmehr Gemeinsamkeiten zwischen den Geschlechtern bestehen. Es gilt also immer Unterschiede und Gemeinsamkeiten in den Blick zu nehmen. Dies zeigte sich z.B. bei der Befragung von Probandinnen und Probanden zur Testung eines tragbaren PDA (Personal Digital Assistant) auf der Bundesgartenschau 2006 (BuGa) in München. Der so genannte Buga-Butler stellte Informationen für die Besucherinnen und Besucher der BuGa bereit. Zu Beginn der Produktentwicklung waren die Forscher und Forscherinnen davon ausgegangen, dass die weibliche Hauptnutzerinnengruppe Frauen zwischen 50 und 65 Jahren mit wenig technischen Erfahrungen sind. Im Verlauf der Untersuchung stellte sich heraus, dass die weiblichen Nutzerinnen des Buga-Butlers eher der Gruppe der technikaffinen Frauen zuzurechnen waren. Die Gemeinsamkeiten zwischen weiblichen und männlichen Nutzern bezüglich der Technikaffinität waren größer als erwartet. 


\section{Gender und Innovation}

Innovations- und Gender-Forschung kommen unabhängig voneinander zu zum Teil vergleichbaren Einschätzungen bezüglich des Veränderungsbedarfs in der industriellen Forschung- und Technikentwicklung. Doch stehen die beiden Stränge bisher unverbunden nebeneinander. Gender-Forschung findet zumeist ohne Einbezug von Ergebnissen der Innovationsforschung statt; die Innovationsforschung wiederum integriert nicht die Gender- und Diversity-Dimension.

Zumeist wird der Begriff des Innovationsprozesses im Innovationsmanagement im weiten Sinne gebraucht und umfasst sowohl die gesamte F\&E als auch das Anfahren der Produktion und die erfolgreiche Markteinführung eines neuen Produktes oder einer neuen Dienstleistung. Aus dieser Perspektive ist die anwendungsorientierte Forschung und Technikentwicklung in der Fraunhofer Gesellschaft, die Gegenstand unseres Projekts war, die erste Phase eines Innovationsprozesses. Dabei kann es sich bei der Innovation um eine objektive Neuheit bzw. Weltneuheit handeln, wenn die Ergebnisse erstmals in dieser Form entwickelt wurden. Eine subjektive Neuheit bzw. Betriebsneuheit liegt dagegen vor, wenn das Ergebnis nur für die Entscheidungsträger und Entscheidungsträgerinnen neu ist (Specht et al. 2002: 14).

\section{„KundInnen“- und Marktorientierung}

Bei der Frage nach der geeigneten strategischen Ausrichtung des Innovationsmanagements wird oft diskutiert, ob grundsätzlich eine ,Technology Push“-Strategie oder eine ,Demand Pull“-Strategie wichtiger ist. Dabei wird unter „Technology Push“ verstanden, ein latent vorhandenes Bedürfnis potenzieller Kunden und Kundinnen durch die Entwicklung einer Innovation zu wecken. Die „Demand Pull“ Strategie geht dagegen davon aus, dass Entwicklungsaktivitäten durch die Nachfrage am Markt induziert werden und daher vornehmlich an dessen Anforderungen zu orientieren sind. Die bisherigen Studien zu den Erfolgsfaktoren von Innovationsprojekten zeigen, dass insbesondere bei Projekten mit inkrementalem Neuheitsgrad die marktorientierte Strategie sich als erfolgreicher erwiesen hat als die technologieorientierte Strategie. Dagegen spielt insbesondere bei radikalen technischen Neuerung die technologieorientierte Strategie ebenfalls eine wichtige Rolle (Specht et. al 2002: 32). Grundsätzlich lässt sich aber auf Basis des jetzigen Forschungsstandes das Fazit ziehen: Es besteht ein positiver Zusammenhang zwischen Markterfolg und Kundenorientierung, auch beim Management der frü- 
hen Innovationsphasen, d.h. auch für Forschungs- und Entwicklungsprozesse (Lüthje 2003: 37).

Genau diese Kundenorientierung ist ein wichtiger Anknüpfungspunkt für die Integration von Gender-Aspekten in anwendungsorientierte Forschungs- und Technikentwicklung: Die Kundenorientierung schärft das Bewusstsein dafür, bereits in der Technologie- und Produktentwicklung den Blick frühzeitig auf unterschiedliche Kundengruppen zu richten, ihre Zugangsweisen und Präferenzen zu analysieren und den jeweiligen sozialen oder kulturellen Nutzungskontext einzubeziehen. Unter den Bedingungen der Globalisierung verändern sich die Märkte zum Teil rapide, aber auch andere soziale und ökonomische Entwicklungen beeinflussen und beschleunigen den Wandel. Dazu gehört nicht zuletzt der Wandel des Geschlechterverhältnisses, der sich zur Zeit in allen großen Industrienationen vollzieht und in Deutschland u.a. an der wachsenden Bildungs- und Erwerbsbeteiligung von Frauen abzulesen ist, und an der seit Jahren intensiv geführten Debatte um die neue, partnerschaftliche Rollen- und Aufgabenverteilungen zwischen Frauen und Männern.

Dabei lassen sich in Bezug auf Gender drei markrelevante Trends feststellen, die in anwendungsorientierter Forschung und Technikentwicklung relevant sein können (vgl. Brühl/Westphal 2004; Horx 2003; Jaffé 2005):

- Der Bedeutungszuwachs von Kundinnen und Konsumentinnen: Die Marktmacht von Frauen steigt und damit steigt die Notwendigkeit, sich mit ihren Präferenzen auseinanderzusetzen. Studien zeigen, dass Frauen häufig sehr viel höhere Ansprüche an das Design, die Nutzungsfreundlichkeit von Technik und den Service technischer Dienstleistungen haben (vgl. Horx 2003).

- Das Aufbrechen traditionell weiblicher und männlicher Märkte: Die traditionelle Aufteilung - Frauen entscheiden über die Konsumgüter des täglichen Bedarfs und Männer über die größeren Anschaffungen - gilt heute nicht mehr. Frauen erobern die Märkte für Technikprodukte und Männer werden in ehemals traditionellen Frauenmärkten, wie z.B. der Kosmetikindustrie, zu einer relevanten Größe. In Paarbeziehungen und Familien wird heute ein immer größerer Teil der Kaufentscheidungen gemeinsam getroffen. D.h. auch hier verändern sich die Entscheidungsmuster, nach denen technische Lösungen am Markt nachgefragt werden. ${ }^{2}$

2 U.a. hat das in Deutschland, Österreich und der Schweiz ansässige Marktforschungsinstitut SevenOne Media eine Studie zu den veränderten Lebens- und Konsumgewohnheiten von Frauen und Männern unter dem Titel „Trendreport Frauen“ herausgebracht und dabei vor allem die Bereiche 
- Die Individualisierung und Ausdifferenzierung von Kundenbedürfnissen: Der Trend geht weg von reinen „Frauen“- oder „Männer“Produkten zur Individualisierbarkeit und Anpassungsfähigkeit von Produkten. Es gilt, sich der vielfältigen Lebensentwürfe und -formen von Frauen und Männern bewusst zu werden und Klischees und Stereotype zu vermeiden. Nur ein Beispiel dafür ist der Bereich der Heimwerker- und Baumärkte, in denen Frauen mittlerweile knapp die Hälfte der Kunden stellen und neuartig gestaltete Werkzeuge mit geringerem Gewicht und für kleine Hände (z.B. Bohrmaschinen oder Akkuschraubenzieher) große Markterfolge erzielen und zwar auch bei Männern. ${ }^{3}$

Im Projekt „Gender-Aspekte in der Forschung“ lag deshalb auch ein Schwerpunkt im Bereich der Marktanalyse mit der Frage, welche Methoden und Instrumente notwendig sind, um die neuen Trends $\mathrm{zu}$ einer komplexeren und differenzierteren Lebenssituation von Frauen und Männern tatsächlich erfassen und in Forschungs- und Technikentwicklung integrieren zu können (für eine Vertiefung sei hier auf den Sammelband „Gender als Innovationspotenzial in Forschung und Entwicklung“" von Martina Schraudner und Helga Lukoschat verwiesen).

\section{Interdisziplinarität und Vielfalt}

Es existiert ein weiterer wichtiger Anknüpfungspunkt in der aktuellen Diskussion um die Innovationsfähigkeit von Organisationen, der die Integration von Gender-Aspekten sinnvoll erscheinen lässt. Es besteht unter Innovationsmanagement-Expertinnen und -Experten Einigkeit darüber, dass Perspektivenvielfalt und Interdisziplinarität in Forschungsund Entwicklungsteams eine der wichtigsten Grundvoraussetzungen ist um Innovationen hervorzubringen. In zahlreiche Veröffentlichungen in den letzten Jahren wird die vielfältige Zusammensetzung von F\&E- und Innovationsteams als Erfolgsgeheimnis bezeichnet und es wurden zahlreiche Methodiken zur Integration von Perspektivenvielfalt entwickelt (vgl. z.B. Kelley 2001; Kelley 2005; Schnetzler 2006; Peters 2003). Die Gender-Forschung kann dazu einen wichtigen Beitrag leisten: Zahlreiche Studien weisen nach, dass bei homogen zusammengesetzten For-

Automarkt, Telekommunikation, E-Commerce und Finanzen in den Blick genommen. (SevenOne Media 2006).

3 In der erwähnten Studie „Trendreport Frauen“ wird eine von dem Baumarkt Hornbach in Auftrag gegebene Forsa-Untersuchung erwähnt, in der deutlich wird, dass Frauen sich mittlerweile eine Vielzahl von Heimwerkeraktivitäten zutrauen (SevenOne Media 2006: 51). 
scherteams, die keinerlei Methodiken zur Perspektiverweiterung einsetzen, die Gefahr besteht, dass Ergebnisse produziert werden, die nur ungenügend den Anforderungen des Marktes und des realen Anwendungskontextes entsprechen. Daran anknüpfend sind in der Gender-Forschung Grundsätze und Methodiken entwickelt worden, um mehr Perspektivenvielfalt in den Forschungsprozess zu integrieren (vgl. z.B. Buhr/Helmers 1994; Wajcman 1994; Schiebinger 2000; Maaß et al. 2002).

Grundsätze gendersensibler Methoden Zusammenfassend kann aus der Gender-Perspektive für die Technikentwicklung festgehalten werden, dass sich - neben definierten Zielen - Annahmen über Benutzer und Benutzerinnen, Benutzungsweisen und Nutzungskontexten in den technischen Lösungen niederschlagen. Diese Annahmen sind nicht selten - implizit oder explizit - von geschlechtsstereotypen und diskriminierenden oder benachteiligenden Vorstellungen über die Geschlechter geprägt. Diese Vorstellungen fließen in die Designentscheidung ein. Die technischen Lösungen ihrerseits setzen damit Rahmenbedingungen, strukturieren Nutzungsweisen und können auf diese Weise die traditionelle Arbeitsteilung fortschreiben. Technikentwicklung dagegen, die vielfältige Nutzungskontexte explizit berücksichtigt, kann neue Nutzungsoptionen entwickeln und die Qualität und Bedarfsgerechtigkeit von Lösungen erhöhen. Damit können Diskriminierungen abgebaut und zum Teil sogar neue Markt- und Innovationspotenziale erschlossen werden.

Daran anknüpfend wurden im Projekt „Discover Gender“ Methodiken aus der Innovationsforschung und der Gender-Forschung erprobt und weiter entwickelt, die bei den im letzten Kapitel beschriebenen Herausforderungen Abhilfe schaffen können. Dabei destillierten sich folgende Grundsätze einer gendersensiblen und innovationsförderlichen F\&E heraus:

\section{Präferenzen der Zielgruppen analysieren}

Bei anwendungsorientierten Forschungsvorhaben und Produktentwicklungen wird zumeist erst viel zu spät analysiert, welche Personengruppen als Adressaten bzw. künftige Nutzerinnen und Nutzer der Produkte und Dienstleistungen in Frage kommen und wie deren Präferenzen genau aussehen. Hier zeigen Untersuchungen, dass sich die von Forschern und Forscherinnen antizipierten Präferenzen überproportional häufig an den eigenen Bedürfnissen orientieren und oft nur die Perspektive des vollerwerbstätigen, weißen, männlichen Akademikers mittleren Alters widerspiegeln. Forscher und Forscherinnen und Entwickler und Entwicklerinnen arbeiten häufig immer noch mit unüberprüften Annahmen 
über ihre Kundinnen und Kunden. Je genauer und realitätsbezogener aber diese Charakterisierung erfolgt, desto zielgenauer kann das Forschungs- und Entwicklungsdesign aufgebaut werden. Unter der GenderPerspektive ist zu prüfen, ob Frauen und Männer in der Zielgruppe vertreten sind und welche vielfältigen Bedürfnisse und Präferenzen sich daraus ergeben. Hier stehen insbesondere aus dem Innovationsmanagement zahlreiche Methodiken bereit - von Empathic Design über Focusgruppen bis zu Lead-User-Methodiken - die unter Einbezug von Gender- und Diversity-Aspekten angewandt werden können (vgl. Schraudner/Lukoschat 2006).

\section{Anwendungs- und Nutzungskontext analysieren}

Bei der Analyse des Anwendungs- und Nutzungskontextes eines zu entwickelnden Produktes/Prozesses können Gender-Aspekte wirksam sein. Entsprechend berücksichtigt die Gender-Perspektive, in welchen Lebens- und Arbeitsbereichen und auf welche Weise Männer und Frauen das Produkt bzw. die Technologie bereits tatsächlich anwenden und welche Nutzungsoptionen ein Produkt oder eine Technologie darüber hinaus für Frauen und Männer zukünftig eröffnen könnte. Unsere Befragung von Expertinnen im Rahmen des Projekts ergab, dass hier bisher vornehmlich Nutzungskontexte aus der Erwerbssphäre und der Freizeit in den Blick genommen werden. Die Nutzungskontexte von Familienarbeit, Kinderbetreuung, Pflege älterer Angehöriger etc. werden bislang vernachlässigt.

Auch die gesellschaftlichen Rahmenbedingungen, die ja Anwendungsund Nutzungskontexte maßgeblich prägen, wie z.B. der Rollenwandel der Geschlechter, die zunehmende Erwerbstätigkeit von Frauen, die demografische Entwicklung etc., werden noch zu selten berücksichtigt.

In diesem Zusammenhang gilt auch, dass das Wissen aus sozial-, wirtschafts- und geisteswissenschaftlichen Disziplinen sehr fruchtbar für die Natur- und Ingenieurwissenschaften sein kann. Inter- und Transdisziplinarität erhält daher eine wachsende Bedeutung.

\section{Partizipative Methodiken}

Ein weiteres Potenzial zur Steigerung der Qualität und der InnovationsFähigkeit von Forschung und Entwicklung stellt der frühzeitige Einbezug der Nutzerinnen und Nutzer in den Forschungs- und Entwicklungsprozess dar. Hier gibt es mittlerweile eine Vielzahl an Methodiken, die aber in den seltensten Fällen angewandt werden, um dezidiert die Bedürfnisse von Frauen und Männern zu erfassen. Partizipative und gendersensible Entwicklungsmethoden stellen ein wichtiges Instrumentari- 
um dar, um das Know-how zur Verbesserung von Prozessen und Produkten von Nutzerinnen und Nutzern besser in den F\& E-Prozess zu integrieren. Gendersensible, partizipative Verfahren gehen aber über die Integration von Nutzern und Nutzerinnen hinaus. Die Integration der Erkenntnisse partizipativer Verfahren in Instrumente des Innovationsmanagements kann hier interessante Impulse liefern, denn partizipative Methodiken beziehen alle von den Ergebnissen betroffenen Beteiligten ein und berücksichtigen Faktoren wie die Kommunikationssituation zwischen den Projektbeteiligten und die jeweilige Organisationsstruktur und -kultur. So können beispielsweise in einem Workshop bei der Neuentwicklung einer Software für eine Organisation nicht nur die unmittelbaren Auftrageber (zumeist das Management) einbezogen werden, sondern alle, die direkt oder indirekt von der neuen Software betroffen sein werden. Dies können die Kundinnen und Kunden der Organisation sein, die Anwenderinnen und Anwender der Software aber auch andere Beschäftigte, die über Erfahrungswissen verfügen, das für die Entwicklung der Software relevant ist. Hierdurch kann die Passgenauigkeit und Bedarfsgerechtigkeit von Produkten und Prozessen verbessert werden.

Dennoch sollte die Perspektive der Nutzer und Nutzerinnen nicht verabsolutiert werden. Denn auch die Nutzer und Nutzerinnen sind geprägt durch soziale Strukturen in der Organisation und in der Gesellschaft und durch symbolische und kulturelle Faktoren (vgl. Hammel 2003: 59). Ein vergleichbares Argument wird in der Innovationsforschung diskutiert, wenn darauf hingewiesen wird, dass die Erfassung der Bedürfnisse von Nutzern und Nutzerinnen nicht ausreicht, um neue Märkte zu erschließen, weil Nutzer und Nutzerinnen häufig nur das sehen und beschreiben, was sie bereits kennen und gewohnt sind (Herstatt/Verworn 2003: 42f.). Daraus folgt, dass die Grenzen partizipativer Methodiken dort beginnen, wo Geschlechtsstereotype von den Nutzern und Nutzerinnen selbst reproduziert werden. Um einerseits Diskriminierungen abzubauen und andererseits Visionen von neuen Feldern zu entwickeln, bedarf es immer der differenzierten Reflexion und Weiterentwicklung auch der Kategorie Gender durch den Forscher und die Forscherin. 


\section{Fazit}

Bisher existieren nur wenige Forschungsprojekte, die sich mit der inhaltlichen Gender-Dimension im Bereich anwendungsorientierter Forschung und Entwicklung auseinandergesetzt haben. Notwendig sind mehr Forschungsergebnisse und Good Practice-Beispiele, die den fachlichen und wissenschaftlichen Nutzen der Berücksichtigung von Gender-Aspekten fachspezifisch nachweisen und veranschaulichen. Für die anwendungsorientierte Forschung und Entwicklung konnte das Projekt „Discover Gender" hier erste Ergebnisse generieren.

Die Erfahrungen des Projekts zeigen, dass die Erfassung der Genderund Diversity-Perspektive sehr gut über folgende Maßnahmen erfolgen kann:

- den Einbezug aktueller Ergebnisse der Gender- und Diversity Forschung,

- den direkten Einbezug von Betroffenen durch partizipative Methodiken (z.B. in Innovationswerkstätten oder Nutzer- und Nutzerinnenworkshops) und

- durch den Einsatz von Methodiken aus dem Innovationsmanagement und dem Innovationsmarketing (z.B. „empathic design“ oder LeadUser-Methodiken), die insbesondere unter Einbezug von Genderund Diversity-Aspekten angewandt werden.

Ziel eines gender- und diversitysensiblen Innovationsmanagements ist nicht allein, die Perspektive von Frauen stärker in den Prozess zu integrieren, sondern auch die Vielfalt an Bedürfnissen und Erwartungen an neu entwickelte Produkte, Prozesse und Dienstleistungen seitens der Kunden und Kundinnen in den Blick zu nehmen und zu berücksichtigen. So können Produkte und Dienstleistungen an neue Bedürfnisse angepasst und Fehlentwicklungen vermieden werden. Innovationen erhalten eine höhere Marktakzeptanz und es lassen sich neue Marktpotenziale erschließen. Die Individualisierbarkeit und Bedarfsgerechtigkeit der Lösungen wird verbessert und Forscherinnen und Forscher erschließen neue, vielfältige Nutzungsoptionen. 


\section{Literatur}

Buhr, Regina (Hg.) (2006): Innovationen - Technikwelten, Frauenwelten, Berlin.

Buhr, Regina/Helmers, Sabine (1994): Corporate Story-Telling. The Buxomly Secretary, a Pyrrhic Victory of the Male Mind. In: Scandinavian Journal of Management, Vol. 10, Nr. 2, S. 175-191.

Brühl, Kirsten/Westphal, Susanne (2004): Female Forces - Der Megatrend Frauen und seine Auswirkungen auf Konsum und Business. Studie der Zukunftsinstitut GmbH Kerkheim, München.

Europäische Kommission (Hg.) (2006): Women in science and technology - the business perspective, Brüssel.

Deutsche Telekom-Stiftung (Hg.) (2005): Innovationsindikator Deutschland 2005 - Ergebnisse einer Studie des DIW, Köln/Berlin.

Genanet (2001): Kurzinformation zu Gender und Nachhaltigkeit, Nr. 5: Mobilität, www.genanet.de.

Hammel, Martina (2003): Partizipative Softwareentwicklung im Kontext der Geschlechterhierarchie, Frankfurt am Main.

Herstatt, Cornelius/Verworn, Birgit (Hg.) (2003): Management der frühen Innovationsphasen. Grundlagen - Methoden - Neue Ansätze, Wiesbaden.

Horx, Matthias (2003): Accent on the Future, Zukunftsstudie von Accenture, Wien.

Jaffé, Diana (2005): Der Kunde ist weiblich, Berlin.

Kelley, Tom (2001): The Art of Innovation, Currency Doubleday, New York.

Kelley, Tom (2005): The Ten Faces of Innovation, Currency Doubleday, New York.

Klima-Bündnis/Stete Planung (2001): Frauen bewegen die Stadt. Daten - Fakten - Argumente, Frankfurt am Main.

Krell, Gertraude (2004): Chancengleichheit durch Personalpolitik, 4. Auflage, Wiesbaden, S. $27 \mathrm{ff}$.

Lüthje, Christian (2003): Methoden zur Sicherstellung von Kundenorientierung in den frühen Phasen des Innovationsprozesses. In: Management der frühen Innovationsphase, hg.v. Cornelius Herstatt/Birgit Verworn, Wiesbaden, S. 35-56.

Maaß, Susanne/Theißing, Florian/Zallmann, Margita (2002): Unterstützung von Interaktionsarbeit im Call-Center. Neue Fragen für die arbeitsorientierte Softwareentwicklung. In: i-com. Zeitschrift für interaktive und kooperative Medien, 3/2002, S. 4-11.

Peters, Tom (2003): Re-imagine! Business Excellence in a Disruptive Age, Dorling Kindersley Limited, London. 
Schraudner, Martina/Lukoschat, Helga (Hg.) (2006): Gender als Innovationspotenzial in Forschung und Entwicklung, Stuttgart.

Schiebinger, Londa (2000): Frauen forschen anders. Wie weiblich ist die Wissenschaft? München.

Schnetzler, Nadja (2006): Die Ideenmaschine: Methode statt Geistesblitz - Wie Ideen industriell produziert werden, Weinheim.

SevenOne Media (2006): TrendReport Frauen, München-Unterföhring.

Specht, Günter/Beckmann, Christoph/Amelingmeyer, Jenny (2002):

F\&E-Management - Kompetenz im Innovationsmanagement, 2. Auflage, Stuttgart.

Wajcman, Judy (1994): Technik und Geschlecht. Die feministische Technikdebatte, Frankfurt am Main. 



\section{A Fascinating History of Curious Careers: Women in Science and Engineering in the Netherlands, $1650-2005^{1}$}

MINEKE BOSCH

In her article „A fascinating history of curious careers: women in science and engineering in the Netherlands“, 1650-2005 Mineke Bosch demonstrates that there have always been women with more than a passing interest in science and technology.

Seventeenth-century Maria Sibylla Merian pursued her research into caterpillar's metamorphosis far into the hinterland of Surinam, while Maria Winkelmann explored the heavens together with her husband for meteors and stars. In the nineteenth-century, as a corollary of widespread educational reform the women's curriculum changed, and women were more and more supposed to dislike the exact sciences. Even so, they kept being interested, though in smaller numbers. In 1976 this led the feminist question „Women in Science: Why so few?“ This question inspired numerous and still increasing activities to promote women's participation in science and technology.

\section{Curious Careers: A Fascinating History}

Women in science and engineering are even today a controversial subject. The low level of interest that girls seem to show in natural sciences and technology in 2006 is a subject that leads to heated discussions.

1 An earlier version of this text appeared in Bosch, Mineke/Oldenziel, Ruth 2006: Curious Careers. An Unexpected History of Women in Science and Technology. Foundation for the History of Technology, Eindhoven. 
Many people believe that the history of women in natural sciences and technology is a straightforward story with few positive episodes. Because even if girls had any chance at all to follow higher education in the past, most people assume they specialized only in typical „women's subjects": modern and classical languages, history, and teaching. Some might even think there is no such thing as a history of women in technology - have women and technology ever gone together? (Oldenziel 1999; Canel/Oldenziel/Zachmann 2000).

Surprisingly perhaps to the casual reader of history, there have always been women with more than a passing interest in physics, chemistry, or biology. Women like Lady Ada Byron Lovelace, who was involved right from the start in the development of computers, and the famous French mathematician Sophie Germain, who in 1816 won the prix extraordinaire. In terms of knowledge and fame, many other women scholars could be competed with the top male scientists of their day. Though compete might not really be the right word here. Many couples, brothers and sisters, and brothers- and sisters-in-law jointly pursued a passion for astronomy, chemistry, geology, or other fields of science as a family enterprise (Schiebinger 1989).

If history is any guide, there is no law that dictates the current small numbers of young Dutch women who choose natural sciences and technology. If we take a look around us we can see that in Turkey, for example, the representation of the sexes in these fields is much better balanced than in the Netherlands. And looking back in time, we even see that the classical languages we consider ,women's subjects“ today traditionally were a must for boys from the elite classes, while for girls these disciplines were taboo. And remarkably given the current calls for women go into the sciences, historically the situation was still quite different, if not the opposite. In other words, the history of girls and women in science and engineering is not a long, bumpy road, but rather it is a varied route with detours and side-roads, with ups and downs, but above all, with lot of unexpected turns. But however we look at it, its history is a story of curious careers. Maybe not always curious in the sense of unusual, but a story about the careers of curious women intrigued by the challenges and pleasures the sciences and engineering had to offer them.

\section{First Scientific Revolution: Challenges and Opportunities, 1600-1700}

The roots of modern science go back to the scientific revolution. It offered new opportunities for women. It also created new detours. When 
we think of the scientific revolution, names readily come to mind like Nicolaus Copernicus, Johannus Keppler, Galileo Galilei, and Isaac Newton, who fundamentally changed our ideas about astronomy and the universe in the first half of the seventeenth century. Physicians increased their knowledge of the human body by dissection and the use of the microscope. Biologists and botanists ventured - literally - into the field to discover, analyze, and categorize new species of animals, plants, and fishes. Geographers and geologists joined the discoverers in the hope of enriching not only the cause of science, but also that of trade.

In 1620, the Englishman Francis Bacon created a theoretical foundation for the new science of ,experimental philosophy“. He believed that the only way in gaining true knowledge was by experimental investigation based on empirical observation. To underline his radical arguments and convince his audience, he relied on recognizable metaphors. He referred, for example, to the "male science" that had to contend with the „female nature“ to gain access to her secrets. And following his successful rhetorical uses, other scholars conjured up similar metaphors - some of them rather violent -, such as the notion that persistent scientists had to conquer and subdue nature or tear off her veil (Merchant 1980; Fox Keller 1985). Such early modern imagery created a symbolic polarization between men as the subjects and women as the objects of science. These were mere metaphors of course. Nevertheless, they did have an effect on the lives and ambitions of curious women interested in the sciences.

The scientific revolution brought benefits to women interested in the pursuit of knowledge. The newly established Royal Society in Britain the first scientific forum for the non-academic pursuit of science from 1660 onwards - might have denied women membership rights until far into the twentieth century, but the new way of learning was certainly not restricted to men alone. Experimental philosophy developed in opposition to the theoretical, classically-based knowledge that was taught at the century-old universities primarily to boys of the ruling classes. These traditional Latin-based universities kept a respectable distance from what they considered the vulgar and practical knowledge of nature used mainly for trading and industry. The practitioners of experimental philosophy, by contrast, advocated the use of national languages to promote the wide distribution of the new knowledge and believed that the new knowledge would bring mankind closer to God.

All these factors benefited the involvement of women in the new experimental philosophy (Phillips 1990). The public lectures-with-experiments, often held with more than a touch of theater, were usually open to people from all social stations. The term "physique amusante“ was 
used for good reason. Contemporary accounts report that the audiences at the spectacular readings of the chemist Robert Boyle included women listeners who often cared for the animals that threatened to expire time after time in the demonstrations of his vacuum pump. And because new scientists published their findings in their own languages, women - obviously without the benefit of a university education - could more easily publish the results of their own scientific investigations and distribute them on a broader basis than would have been the case in classic fields of study.

The natural sciences' novel emphasis on practical knowledge benefited women in yet another way. As the mistress over the household, women could profit from the knowledge of chemistry, meteorology, natural history, and medicine. Thanks to chemistry, housewives could get better results with their washing and gain a better understanding of food preparation; metrology helped women keep their thoughts tidily arranged; and knowledge of nature simplified the preparation of medicines and caring for the sick. Women in the Dutch Republic in the sixteenth and seventeenth centuries in particular had a reputation for their ability to organize things independently of their seafaring men and held up as an example to their English sisters because of their practical knowledge. The British Batshua Makin, head of a girls' school and sister of a member of the Royal Academy, celebrated the „honest, well-bred, ingenious, industrious Dutch-woman" for their knack for enterprise and for the practical application of what was then called ,useful“" knowledge (Phillips 1990).

\section{Anna Maria van Schurman and Maria Sibylla Merian}

Contemporaries called the Dutch woman Anna Maria van Schurman (1607-1678) ,the Pallas of Utrecht“ for her exceptional wisdom and erudition (De Baar 2007). Schurman, born in Cologne, moved with her family to Utrecht at the age of seven. She stood out when she was still very young. In 1625 the renowned poet and statesman Jacob Cats sung her praises as an exceptional woman. „You jewel, just recently arisen, from whose learned youth and distinguished pen, the cities on the Rhine and I have witnessed" (ebd.). On her own accord, Schurman entered into a discussion with a famous theologian, Andreas Rivet, about whether Christianity permitted women to study. That correspondence led in 1638 to Treatise on the suitability of the female spirit for science and letters. The publication attracted the attention of many other highly educated 
women in Europe, including Marie le Jars de Gournay (1565-1645), who had issued a comparable work in 1622 entitled De l'égalité des hommes et des femmes and was also well known for her chemistry experiments.

Unlike many other well-educated women of her time, however, Schurman specialized in the theological debates. Gisbert Voetius, the Utrecht professor of theology and Eastern languages and minister for the reformed community, allowed her to follow his university lectures from a sort of cubicle. Following lectures in literature and medicine in similarly circumscribed conditions, she became effectively the first female student in the Netherlands. Through her brother Johan Godschalk, Schurman came into contact with the reformed preacher Jean de Labadie in Geneva, who sought to return religious practice to ascetism, meditation, and contemplation. When he radicalized further and was put out of office of the Walloon reformed church in Middelburg, Schurman accompanied him and his followers in the Walta State in the village of Wieuwerd, Friesland (Vries 1970).

A generation later, Maria Sibylla Merian (1647-1717) joined the same religious commune and gained such great fame as a highly educated woman that the memories of her have not disappeared even to this day. The tangible signs of her productive life she left behind, including a number of beautiful works on entomology (the study of insects) she compiled and illustrated herself, might have been responsible for that.

Merian was born in Frankfurt in 1647 to a family of craftsmen. „Her father, Matthäus Merian, was a renowned artist and illustrator, who was best known for his lavishly illustrated Merian bible“. He died when she was three, but the man whom her mother, Johanna Heim, remarried, Jacob Marell, was an artist and a member of the artists' guild. In his workshop she learned all the techniques of illustration: drawing and painting, mixing paint, and making copper etchings. After marrying one of her stepfather's pupils, she started selling fabrics she had painted herself.

Her life's work for which she became famous was the book Der Raupen wunderbarer Verwandlung und sonderbare Blumennahrung (1687), that dealt with caterpillars' metamorphosis into chrysalises and butterflies and with the flowers on which they feed. It was based on years of thorough study and contained fifty copper engravings. She made the important discovery that specific caterpillars belong to specific plants, and these then develop into specific butterflies. The work made her so well known that there was an immediate demand for a second volume. In the meantime she published her Neues Blumenbuch in 1680 with 36 engravings intended for readers who needed samples for their paintings and embroidery. 
Around 1685, Merian left her husband to move in with the Labadists in Friesland, only to move again in 1690 to Amsterdam, where she met leading families who opened their cabinets of trophies and objets d'art. Especially the cabinet of the Amsterdam mayor Nicolaas Witsen, with his many trophies from both East and West, made a big impression on her. She also met Casparus Commelin, botanist and founder of the Amsterdam Hortus botanicus, and familiarized herself with the scientific work of Jan Swammerdam and Antoni van Leeuwenhoek, the famous pioneers in the field of microscopy.

The tropical wonders she encountered in Amsterdam awakened her desire to carry out research and draw in Suriname, where both the Labadists community owned plantations and her eldest daughter lived. Financial support from the municipality of Amsterdam enabled Merian to pay for the voyage for herself and her youngest daughter in 1699 . From Paramaribo she sailed the length of the Suriname River to research the hinterland. Four years after returning to Amsterdam in 1701, she published her most famous work: Metamorphosis Insectorum Surinamiensium (Metamorphosis of the Insects of Suriname), at first only in Latin, and later also in a Dutch translation. From that time until her death in 1717, she worked to revise and translate her first two-volume work Der Raupen wunderbarer Verwandlung und sonderbare Blumennahrung. A third volume was published posthumously. Although her work became at least as famous as her father's, her scientific qualities were controversial right from the start because they did not live up to the scientific standards of the time: she first did not publish in the official scientific language of Latin, while later her illustrations were considered to be too beautiful and artistic. Today she is recognized as a major entomologist and scientific illustrator.

\section{Science Books For and By Women, 1700-1800}

Women also benefited from publishing innovations that helped to break the monopoly on the production of knowledge which monasteries and classical universities had traditionally held. The popularization of the new sciences gained a great impulse from books like Urania Practica (1649), the first English astronomy textbook, and Introduction to Astronomy and Geography Being a Plaine and Easie Treatise of the Globes (1675), a real do-it-yourself book. Not long after, the predecessor of a long series of textbooks for women, Bernard le Bovier de Fontenelle's Entretiens sur la pluralité des mondes (1686) appeared in France. In England, Aphra Behn, reputed to be the first female author 
who lived from her writing, published the first translation. Almost as famous as Fontenelle's book was the Italian Francesco Algarotti's much translated, Il Newtonianismo per le dame of 1737, or Benjamin Martin's widely read two-part work Young Gentleman's and Lady's Philosophy of 1763. The first half of the eighteenth century also saw the publication of a number of periodicals aimed specially at women, such as the Ladies Diary, which challenged women to test their skills at „Writing, Arithmetick, Geometry, Trigonometry, the Doctrine of the Sphere, Astronomy, Algebra, with their Dependants, viz. Surveying, Gauging, Dialing, Navigation, and all other Mathematical Sciences“ (Phillips 1990).

Around the end of the eighteenth century, women also started to try their hand as authors of the popular scientific handbook genre. Priscilla Wakefield's An Introduction to Botany of 1796, for example, reached its eleventh reprint in 1841. Her 1805 Domestic Recreations; or Dialogues Illustrative of Natural and Scientific Subjects was even more successful. Another best-selling author was Jane Marcet with her Conversations on Chemistry, intended more especially for the Female Sex (1806), the Conversations on Political Economy (1815), and Conversations on Natural Philosophy (1820).

On their part, Dutch women could imbibe the new knowledge from a mixture of translations and native works (Bosch 1997). Algarotti's Newtonian philosophy for women found his way to the Netherlands in translation in 1768. A very popular edition was the 1859 translation from the French Abbé Nollet's, Lessons in physics, confirmed by experiments. In clarification of all kinds of everyday matters. Remarkably, it showed illustrations of women actively involved in scientific experiments in its first edition. The members of the Ladies' Society for Physics in Middelburg learned their lessons from this book. And reverend J.F. Martinet wrote a very famous work Katechismus der Natuur (1777-1779) in four volumes that was reprinted until well into the nineteenth century. He used it for teaching a broad public, including a women's reading society in Zutphen.

Many works of this kind were advertised as ,per le dame“ or „specially for women“. No doubt they also drew male readers. Fontenelle, for example, was translated by the Leiden professor Johan C. Gottsched, but he deliberately left out every reference to the popular nature of the work - never mind the intended female readership. Jane Marcet's Conversations on Political Economy, translated by another Leiden professor, H.W. Tydeman in 1925, probably also served as the basis for the first lectures in domestic science in the Netherlands. In the translation of another work of Jane Marcet, Tydeman again left out her first name, so that young readers remained ignorant about the true gender of the au- 
thor. Jane Marcet's work about chemistry is reputed to have provided the spark that ignited the chemist and physicist Michael Faraday's love for the subject generations later. Women popular science writers, in short, had a major impact on science practice in the eighteenth century.

\section{The Enlightenment:}

\section{A Double Heritage, 1750-1900}

The Enlightenment age was a double-edged sword for female scientists and engineers. Its promise of universal rights, equality, and liberty paved the way for equal rights and opportunities. The Enlightenment, however, also marked the era that invented and highlighted sexual differences that came to be carved in stone and also would limit the possibilities of women's advancement in science in the long run.

The establishment of both the Paris and Berlin academies of sciences in 1770 and 1666 showed that the exclusion of women, during the first scientific revolution, was neither predetermined nor simply accepted (Schiebinger 1989). In the French academy, a number of women held official functions, and in Paris a salon culture arose, in which male academy members and women continued to meet each other on an equal basis. In the first ten years of the Berlin academy's existence, astronomer Maria Winkelmann (1670-1720) was officially recognized as the assistant of her husband, who was appointed as academy astronomer. At the time, experimental philosophy (in this case astronomy) was still based on a tradition of guilds, in which science was a family business. Yet as a sign of the new times, Winkelmann was passed over as her husband's successor after his death, as had been customary with the widows of guild members, who frequently continued their husbands' businesses. Increasingly in the field of science and outside it the lines between the sexes were being drawn ever more clearly in the Age of Enlightenment.

We generally view the Enlightenment as the origin of liberal Western values such as rationality and reason and a belief in progress, universal human rights, and democracy. For women that did not always pan out in the same fashion. Some contested the commonly held views about women, including the relatively unknown French feminist and Cartesian philosopher François Poulain de la Barre, who in his treatise The Equality of the Sexes (1673) argued that „The Mind has no Sex“. These thinkers, however, found fierce opposition from such philosophical heavyweights as Jean-Jacques Rousseau and Immanuel Kant. Rousseau, who believed a woman is a man in every respect except for her sex, argued however that her sex determined her behavior: „the female is female all 
her life“. Immanuel Kant called on universal „man“ (Mensch) to emancipate himself from his self-imposed constraints. In his series of lectures entitled Anthropology, however, he followed Rousseau arguing that emotions and married life dictated and ruled women, effectively excluding women from the universal category of ,man“.

Most thinkers derived their views of men and women from the great numbers of anthropologies that, in contrast to today's practice, were based largely on medical-anatomical research. This research increasingly regarded the natural difference between the sexes as immutable. It presented that difference as pervading the entire person. On the basis of that new logic, men were designated as active, rational, resolute, and productive; women as passive, emotional, fickle, and trivial. „For him the world, for her the home", was the rule that from then on influenced public and private life. It also shaped the organization of science. Increasingly, the practice of science became a matter of individual men making their heroic discoveries within public institutions rather than the family businesses in the private sector.

\section{The Middelburg Ladies' Society for Physics, 1785-1887}

When the library and possessions of the society Lady Hillegonda Catharina Schorer of Middelburg were auctioned in 1821, they also included an „electrifying machine“, with which an „electric kiss“ could be given in salon demonstrations (Sturkenboom 2004). This was done by charging a man with static electricity using an electrostatic apparatus. Then a woman from the audience was invited to place her mouth so close to the man's that a large spark jumped over to her. The „electric kiss" symbolized the natural sciences of that period, in which learning and entertainment went hand-in-hand. Experiments were shown to mixed groups of men and women in drawing-room settings. Natural science presentations, however, increasingly moved to gatherings of the numerous societies that sprung up in the eighteenth century and that, with very few exceptions, were accessible only to men. The Ladies' Society for Physics in Middelburg (in the province of Zeeland, the Netherlands), founded in 1785, represents one of the few ladies' scientific societies of the time.

The prominent citizen Adriaan van de Perre founded the society. $\mathrm{He}$ invited 44 women from the elite classes in Middelburg, who registered as members in 1785. A regular speaker in the early days was reverend Ballot, the father of the famous meteorologist Buys Ballot, who dealt 
with Abbé Nollet's work. According to the proceedings, the Middelburg ladies were taught physics in a contemporary format, in which a „knowledge of Nature“ was believed to lead to a deeper understanding of the majestic work of the Creator.

The „Physics for Ladies“ as practiced in Middelburg show that the relationship between women and natural sciences went through a period of warm mutual friendship, which did not cool down until far into the nineteenth century. A century later, around 1887, that love had faded, crowded out in part by the increasing influence of voices maintaining that women had no talent for the exact sciences. The shifts in education for girls reflected a similar change. Initially, „the sciences“ (meaning the practical, natural sciences) had formed a large part of most Dutch teaching programs at the so-called French schools. The foundation of the Girls' Secondary School, however, brought a marked decline of the share of the exact sciences in the curriculum for girls. The window of opportunity that had been opened for girls was closing again.

\section{Second Scientific Revolution and the Changing Curriculum, 1850-1950}

The nineteenth-century changes in natural sciences mark the second scientific revolution. It increased the importance of the natural sciences in the university curriculum, helped to develop the scientific researcher into a professional, and announced the specialization of research. Researchers started to demand laboratories that were equipped with the latest instruments for carrying out experiments. These developments led to a relocation of scientific research from the private to the public domain.

Subsequently, educational reforms were taking place everywhere in the Western world. In the Netherlands, the Latin School transformed into the Gymnasium that paid attention to the classical languages, modern languages, and natural sciences, and to the establishment of higher secondary school for boys from the middle classes (the HBS). The curriculum emphasized „modern“ subjects such as the natural sciences and the living languages to prepare boys for positions in trade and industry. The HBS was the jewel in the crown of the Education Act the liberal statesman J.R. Thorbecke drafted. These new schools were generously supported with innovative teaching materials. A well-equipped physics and chemistry laboratory was standard. The new high schools often maintained various collections and a garden. Although Thorbecke had not at first thought of it, he responded to debate in the Dutch parliament and the girls' education movement, pressing him to consider top open up 
secondary education for girls from the higher social classes. He was prepared to offer the legal framework of the Girls' Secondary School, but he believed its organization and financing was not the duty of the state, but should come from citizen initiatives (Bosch 1994).

Women's education advocates argued girls should have the opportunity of further personal development, but also they debated what its purpose should be: life as a housewife, motherhood and/or a career, and in which way. As elsewhere in Europe, Dutch education reformers mainly answered the question about the curriculum in a „modern“ way: women should be housewives and mothers. Only a minority maintained that women should prepare for an independent life. As an unintended consequence the „sciences“ became less valued. It represented a break with the first half of that century. But just as the HBS started to send students to the universities held as the sole right of the Gymnasium, young women also started to show an interest for these secondary education institutions, and even for the university.

Aletta Jacobs was the first woman in the Netherlands to register as a medical student, at the University of Groningen in 1871; she graduated in 1878 and earned her doctorate in 1879 (Bosch 2005). Although she encountered virtually no resistance, compared with girls and women in England or Germany, few followed Jacobs' example. Although increasing numbers of women started to show an interest in attending university lectures or registered at university to gain a secondary education diploma, it was Aletta Jacobs' sister Charlotte who in 1882 was the second woman in Holland to complete her academic studies with a degree in pharmacy. From that time until 1898, there was a slow but steady increase in the numbers of women students, many of whom had not registered for a complete study program or intended to see their studies right through to completion. Remarkably perhaps from today's effort to encourage women to pursue science and engineering, in this earlier period young women showed a strong preference for studying mathematics, physics and medicine (Kirejzcyk 1993; Bosch 2002). 
Table 1: Dutch Women Students per Faculty per Year (Totals and Percentages)

\begin{tabular}{|c|c|c|c|c|c|c|c|c|c|}
\hline & 1898 & & & 1928 & & & 1940 & & \\
\hline & Total & $\begin{array}{l}\text { Wom- } \\
\text { en }\end{array}$ & $\%$ & Total & $\begin{array}{l}\mathrm{Fe}- \\
\text { male }\end{array}$ & $\%$ & Total & $\begin{array}{l}\mathrm{Fe}- \\
\text { male }\end{array}$ & $\%$ \\
\hline Theology & 393 & 1 & & 678 & 42 & 6,2 & 779 & 71 & 9.1 \\
\hline Law & 476 & 5 & & 1,590 & 262 & 16,5 & 1,610 & 430 & 26.7 \\
\hline $\begin{array}{l}\text { Law and } \\
\text { Arts }\end{array}$ & & & & 337 & 15 & 4,5 & 375 & 49 & 13.1 \\
\hline Medicine & 1,126 & 29 & 2,3 & 3,125 & 374 & 12 & 3,470 & 634 & 18.3 \\
\hline $\begin{array}{l}\text { Math and } \\
\text { Natural } \\
\text { Science }\end{array}$ & 402 & 48 & 11,9 & 1,862 & 508 & 27,3 & 1,437 & 418 & 29.1 \\
\hline $\begin{array}{l}\text { Geogra- } \\
\text { phy and } \\
\text { Psychol- } \\
\text { ogy }\end{array}$ & & & & 57 & 15 & 26,3 & 112 & 52 & 46.4 \\
\hline $\begin{array}{l}\text { Philoso- } \\
\text { phy and } \\
\text { Literature }\end{array}$ & 178 & 18 & 10,8 & 1,224 & 561 & 45,8 & 865 & 380 & 43.9 \\
\hline $\begin{array}{l}\text { Econom- } \\
\text { ics }\end{array}$ & & & & 159 & 22 & 13,8 & 190 & 18 & 9.5 \\
\hline $\begin{array}{l}\text { Veteri- } \\
\text { nary Sci- } \\
\text { ence }\end{array}$ & & & & 158 & 1 & 0,5 & 135 & 7 & 5.2 \\
\hline $\begin{array}{l}\text { Technol- } \\
\text { ogy and } \\
\text { Agricul- } \\
\text { ture }\end{array}$ & & & & & $113(*)$ & & 2,778 & 119 & 4.3 \\
\hline Total & 2,716 & 101 & 3.7 & 9,561 & 1,810 & 18.9 & 1,1251 & 2,044 & 18.2 \\
\hline
\end{tabular}

Source: Jensma and de Vries 1997: 193, 204, 210.

Source (*): Freie 1948

\section{Never-ending Debate about Women and Science, 1870-now}

The Dutch public debate about women's education received a major impulse from the organization of the National Exhibition for Women's 
Work at Scheveningen in 1898. The event provided a platform for a number of congresses, dealing with a host of topical subjects and attracted record numbers of visitors. The organizers credited the extensive publicity not least to the high-level attention the president Cécile Goekoop-de Jong of Beek en Donk had drawn in the preceding year to women's issues with her best-selling novel and social commentary Hilda van Suylenburg (1898). The main roles in this fascinating and wide-ranging feminist pamphlet were played by a doctor and a woman lawyer. Perhaps not coincidently, the year following the bestseller publication the number of women students suddenly increased by leaps and bounds, continuing to show a non-linear increase to $19.8 \%$ in 1970 (in 1960 it was $17.9 \%$, in $194018.2 \%$ and in $192818.9 \%$ ).

The high level visibility of women's demands sparked a neverending debate about „women's studies“ in 1898. „Are women suited to studying?" the Amsterdam gynecologist Hector Treub famously wondered during a lecture to a feminist women's society. „Yes“, was his resolute answer: studying is suited to women, and women are suited to studying. But that did not bring about a revolution. The most important tasks for women were still those of marriage and motherhood. His colleague Cornelis Winkler, on the other hand, had a different view. For his argument, he rallied the opinions of numerous colleagues in the USA, England, Germany, and Switzerland. The development of ,women's studies" was getting out of control, he believed. A woman who pursued a scientific career was a freak of nature, or to use the polite or scientific Latin name, a „monstrum“. And a society that did not place limits on this trend ran the risk of degeneration and sterility, he argued (Bosch 1994).

The fierce 1898 debate did not mark the last stage of men's resistance to the integration of women in their „male domain“. Nor was the debate a clear or final answer to those developments. Winkler had earlier indicated the limits that should be placed on what contemporaries called women's studies. As far as he was concerned, women could study medicine to become general practitioners, but not to become professors. He also used the so-called man-woman differences to reinforce the image that research in the sciences naturally suited men. From then on, polemics of this kind continued almost without interruption in different disciplinary contexts and periods, always resulting in a further determination of the (restricted) place of women in science.

The uncharitable view of girl students the influential professor of Dutch national history P.J. Blok expressed in 1909, also played a role in the long-term custom of granting women no more than assistants' positions in academia. At the same time he assessed „real historians“ could 
only be men. In a controversial survey of women's studies, the renowned psychologist and experimental scientist Gerard Heymans relied on „common sense“ notions about women, men, and science to demonstrate the value of psychological research based on interviews. Even so, he confirmed that women could perhaps go a long way on the basis of hard work and applied science, but that their stronger emotions prevented them from making great scientific achievements. The plea for stronger links between science and society in the 1930s, together with a raised status of the practical and useful natural sciences and technology at the expense of the humanities, might not have been primarily about the roles of men and women. Yet, it did have a limiting effect on the numbers of women in science in the Netherlands. Not only were they underrepresented in the sciences, but women students were also expected to study only for their ,general development“", instead of preparing themselves for life-long careers. The post Second World War debate about university education too may not have been primarily about women students' place and ability, but the renewed emphasis on the importance of education resulted in a separate „women's curriculum“, in which there was no longer room for the exact sciences.

The ongoing debate about women's proper place in academia had a chilling effect. The proportion of Dutch women students never exceeded $20 \%$ until 1970. It also had an effect on the ,preference“ of women for specific disciplines. Before 1898 the majority of women showed a preference for the exact sciences and mathematics, and medicine (48 and 29 of 101, respectively). By 1897 only the first law student was enlisted and a year later only 18 women out of 101 registered as students in the humanities. In 1940, the proportion of medicine, and natural sciences and mathematics had declined to 643 and 418, respectively, out of a total of 2044 female students. Women students in the natural sciences and mathematics still accounted for more than $50 \%$ of the total, but still it was a declining trend. Within mathematics and natural sciences, pharmacy was by far the most popular for a very simple reason: as in medicine and dentistry, pharmacists could become self-employed. And many of them did exactly that: in 1930, 153 (out of a total of 544) women pharmacists had their own pharmacies. Self-employment gave these women the ability to determine not only their own working conditions, but also their private lives. In contrast to women in many other professions, most of these pharmacists combined a family with their work. 


\section{Special or Separate? \\ Women in Science, 1870-1970}

The second scientific revolution in the Netherlands has been described as a second Dutch Golden Age, which led to an impressive number of Nobel Prizes (six) at the beginning of the twentieth century (Van Berkel 1998). The increasing professionalizing of science and research, however, did not always favor women scientists in the academic field. Until 1970 , only exceptionally few women pursued scientific careers and only a fraction of them reached the position of professor. The journeys of many of them became bogged down in (head) assistants' positions or in lecturers' positions that were sometimes changed to professorships at the eleventh hour (on reaching the age of 65). As a group, these women remained often (highly appreciated) outsiders, both in scientific and in social terms. Most of the career women scientists remained unmarried, with all the advantages and disadvantages of that status.

Biology forms the exception to this rule. For a long time, welleducated, aristocratic ,amateurs“ like Anna Weber-van Bosse, who gained (as the first woman in the Netherlands) in 1910, at the Utrecht University, an honorary doctorate for her research into algae she had carried out in her own laboratory, had a respected place in the field. Male mentoring of women talent also had an from Utrecht University played a positive part in the appointment of the biologist Johanna Westerdijk as the director of the Willie Commelin Scholten small plant diseases laboratory in Amsterdam in 1906, and later in her establishment as extraordinary professor in 1917 (Bosch 1988; 1994). Two years later, the Groningen professor Willem Moll also demanded recognition for his brilliant student Jantina Tammes in establishing an extraordinary chair at his department (De Wilde 2001).

It is hard to assess the exact role of mentors, nevertheless a number of disciplines and laboratories proved to be fertile ground for Dutch women: the laboratories of the chemical engineer F.E.C. Scheffer at the Delft University of Technology, the entrepreneurial physicist Leonard Ornstein in Utrecht, the gynecologist Hector Treub in Amsterdam, Johanna Westerdijk in Baarn, or the crystallographers J.M. Bijvoet and Caroline MacGillavry in Amsterdam. (Stamhuis/Offereins 1998) Just as important is that the disciplines such as crystallography and radiology maintained an international network in which many women were actively participating. In addition, many women developed specialties all of their own or focused on newly emerging research areas. Jansje Schuiringa for instance made major advances in prosthetic dentistry in Utrecht, while Antonia Korvezee was one of the first researchers in the 
Netherlands to systematically investigate radiation (De Jong 1988; 2004).

Dutch women scientists also developed other strategies to dispense with the institutional constraints of university research by working for government or industry. On the entry level the government and industry employment offered opportunities, but here too academically trained women came up against ,glass ceilings“. There were some notable exceptions though. Neele Wibaut-Isebree Moens was for many years affiliated with the Public Health department of the city of Amsterdam, N. Kloppert for many years carried out research at the Amsterdam municipal Trading Standards Department (Bosch 1994). Starting one's own business, finally, offered academically trained women alternatives to the limited possibilities for career advancement at universities, the government, or business. There have been enterprising women who continued their research work in their own laboratories, possibly with profits in mind. That was the case with the physicist Carolina Bleeker. Her firm at one point even had a workforce of 150. Delft trained chemical engineers Hilda Vormer-Roosenstein and Hendrina de Wijs who were strongminded enough to establish their own research laboratory and consultancy practice with which they were able to earn a living. 
Table 2: Women Academic Scientists in the Netherlands 1948 (Universities and higher vocational-economical, technical, agricultural-education)

\begin{tabular}{|c|c|c|c|c|c|c|c|}
\hline & $\begin{array}{l}\text { Profes- } \\
\text { sor }\end{array}$ & $\begin{array}{c}\text { Lector } \\
\text { (Associate } \\
\text { Professor) }\end{array}$ & Other & $\begin{array}{c}\text { Total } \\
\text { univer- } \\
\text { sity }\end{array}$ & $\begin{array}{l}\text { M.A. } \\
\text { (Drs.) }\end{array}$ & Ph.D. & Dr. \% \\
\hline Theology & & & & & 98 & 7 & 7.1 \\
\hline Law & 1 & & 2 & 3 & 1,381 & 160 & 11.5 \\
\hline Medicine & 1 & 3 & 12 & 16 & 1,354 & 108 & 7.9 \\
\hline $\begin{array}{l}\text { Mathematics } \\
\text { and Natural } \\
\text { Science }\end{array}$ & 3 & $5(+1 *)$ & 41 & 49 & 1,106 & 204 & 18.4 \\
\hline Biology* & 1 & 2 & 22 & 25 & 239 & 100 & 41 \\
\hline Pharmacy* & 1 & 2 & 4 & 7 & 596 & 22 & 3.6 \\
\hline $\begin{array}{l}\text { UF2: Arts and } \\
\text { Natural Sci- } \\
\text { ence }\end{array}$ & 2 & 1 & 3 & 6 & 134 & 22 & 16.4 \\
\hline $\begin{array}{l}\text { Literature and } \\
\text { Philosophy }\end{array}$ & 4 & $4\left(+5^{*}\right)$ & 24 & 32 & 1,117 & 243 & 21,8 \\
\hline Economics & & & & & 45 & 1 & 2 \\
\hline $\begin{array}{l}\text { Veterinary } \\
\text { Science }\end{array}$ & & & & & 8 & 1 & 12.5 \\
\hline $\begin{array}{l}\text { Technology } \\
\text { and Agricul- } \\
\text { ture }\end{array}$ & & 1 & 2 & & 247 & 12 & 4.8 \\
\hline Total & $11(+2 * *$ & $14\left(+6^{* *}\right)$ & 84 & 109 & 4,109 & 598 & 14.5 \\
\hline
\end{tabular}

Source: Van der Kolf 1950.

* Only biology and pharmacy in this table are shown specifically; they are included in the overall figures for mathematical and natural science. ** Educated in the Netherlands; Appointed at foreign universities. 


\section{A 1966 Feminist Challenge: Why So Few?}

During the 1960s, academically educated women everywhere in the Western world started to discuss the ,problem without a name“ women's hard-to-assess feelings of dissatisfaction with a life that relegated them to the realm of housewives and mothers, or to secondary positions in the public sphere. They started to claim that ,the personal is political", arguing that women's private lives were valuable and should be understood at a political level. Fifteen years earlier, Simone de Beauvoir's classic essay Le Deuxième Sexe on how women were made into „the second sex", had impressed a handful of women. Now it became one of the key texts of the second wave of feminism inspiring a whole generation of young women. De Beauvoir posited that women are made into women by an overwhelming and non-stop production of knowledge about women, from philosophical texts and biological evidence, to popular cultural notions and literary works. Language and culture were therefore central targets of second-wave feminists.

In the universities, women students and lecturers formed groups who following the example of sociologist Alice Rossi - wondered: „Women in Science: Why so Few?", after Rossi held a speech in 1966 at MIT about women in science. Indeed that question was topical, for the progress of women in the sciences had not really advanced beyond the promising strides during the $1920 \mathrm{~s}$, when so many women had entered academia as students. All over the Western world, the position of women students seemed to be in better shape during the decade of the 1920 s than during the 1960 s. Over time, various answers to this question were formulated.

One of the first was that the content of science had a unilateral male or masculine bias, and that it stereotyped or excluded women. This led to the development of women's studies (later called gender studies). Sometimes organized as a separate unit, it often sought to highlight the „blind spots“ within the existing scientific disciplines and generated new interdisciplinary knowledge. Within the new discipline, a feminist reflection of science itself was undertaken from different disciplinary perspectives: history, epistemology and sociology. This led in the Netherlands to the publication „Cracks in the foundations. Women, Natural Sciences, and Technology" (Vrouwen 1986).

A second answer to the question „Why so Few?" came from the development of a governmental emancipation policy in higher education and science and at the universities. The Dutch government initially responded to the demand for women's studies by awarding several major 
subsidies around 1980. By the end of that decade, a consensus developed that new policy should focus on the entire female scientific staff. For some time this did not go beyond measures to create the desired working conditions such as childcare and maternity leave or training for women. An occasional program with a more far-reaching aim to raising the number of the appointments of professors (affirmative action measures) failed because of the unwillingness of the incumbent academic staff. The universities themselves - in other words their role in the under representation of women in science - remained largely free of blame. Nevertheless, policy makers began focusing on questions such as ,why do women not achieve higher positions as quickly as men?“ or ,why do more women leave their scientific careers?" At the beginning of the 1990s, it also became clear that - despite the second feminist wave - the number of female professors in the Netherlands had declined since the early 1970 s, instead of increased!

It still took until 1997 before the under representation of women scientists received a degree of priority on the agenda of decision-making institutes in the Dutch academic community. Two Swedish medical researchers, Agnes Wold and Chrtistine Wennerås, showed in a study published in Nature supported through hard data that women had to excel twice as well at successfully acquiring specific research funding than men. In response to the study, the European Commission in Brussels decided to make gender equality one of the important pillars of the scientific policy agenda. One of the results was the publication in 2000 of a comparative international European report (the ETAN Report) about the position of women in science by a group of international experts under the title of Research Policies in the European Union: Promoting excellence through mainstreaming gender equality. This showed once again that in international terms the Netherlands did poorly with only $5 \%$ female professors. Finland was on top of this list with $18.4 \%$ professors, followed by Portugal and France with $17 \%$ and $13.8 \%$, respectively. The Netherlands tied with Belgium (5.1\%) and Germany (5.9\%).

This marked the start of a new phase in tackling the under representation of women. (Bosch/Hoving/Wekker 1999; AWT-Avies 2000) The Dutch ministry instructed the Dutch Organization of Research (NWO) to develop the ASPASIA-program in cooperation with the universities. While observing the customary peer review procedures, this program offered women university lecturers the opportunity of acquiring research funding, and when successful, progressing from assistant to the position of associate professor. The ASPASIA-program was a big success. A total of 270 proposals were submitted for the planned two rounds. Impressed by the proposals' high quality and encouraged by the Advisory 
Council for Science and Technology Policy's recommendation, the minister together with the NWO immediately decided to honor not two times 15, but in the first round 30 and in the second round 40 proposals. An almost equally large number of proposals were assessed as ,good“, but could not be honored because of lack of resources. In response to this well of talent, NWO then encouraged universities themselves to appoint these candidates as associate professor and to fund the proposals from their own local resources. Most universities indeed complied with NWO's request. Finally, 146 women were appointed as associate professor as a result of the ASPASIA-program. Between 1999 and 2004, when the second round had been completed, the percentage of female associate professors increased from $9.4 \%$ to approximately $14.5 \%$.

Even more important was the fact that the NWO had shown with this program that measures could certainly be taken to increase the numbers of women appointed while maintaining its quality standards. NWO'S efforts encouraged a number of universities to push and develop their own programs to promote the appointment of qualified women.

\section{Lisbon agreements}

This is all the more important since the Lisbon agreements made by the European Commission to transform Europe into a knowledge economy set a target of $25 \%$ for the percentage of women professors in all member states by 2010 . The Dutch minister of Education, Culture and Science, Maria van der Hoeven, in her memorandum „Research Talent Gets the Valuation it Deserves" felt forced to adjust this figure downwards because she believed $15 \%$ is a more realistic target. The poster below shows what that means the question is: will Dutch universities reach this goal? The poster Making Lisbon Happen was developed by the ESFEqual project participation of Women as Priority for Science. It was issued March 8, 2006. 
Figure 1: Making Lisbon Happen

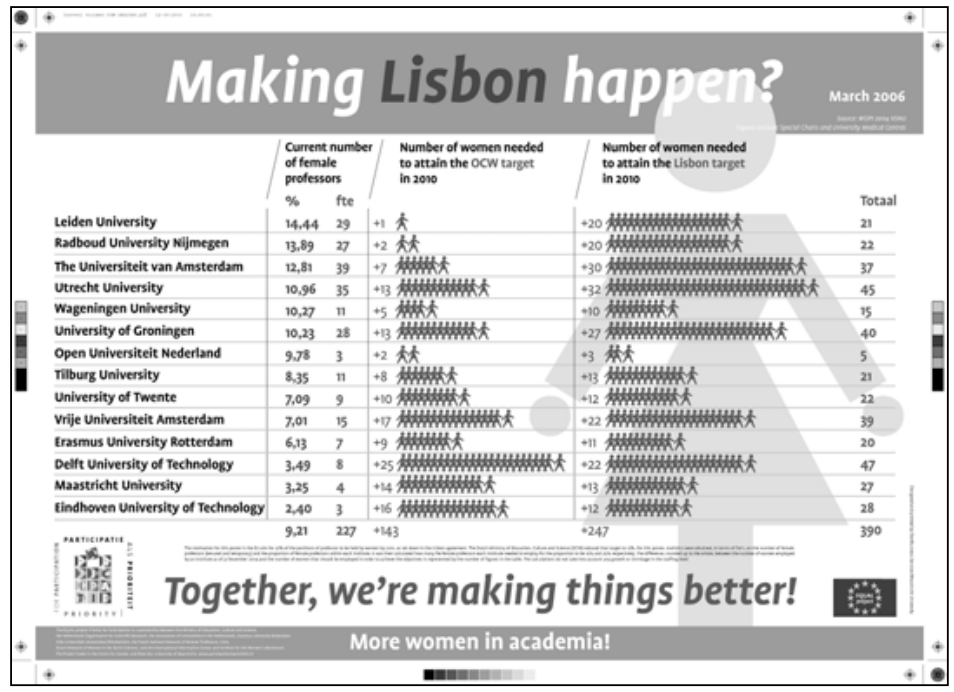

Resource: ESF-Equal project participation of Women as Priority for Science 2006, www.participatiealsprioriteit.nl

\section{Selected Bibliography}

AWT-Advies (2000): Adviesraad voor Wetenschaps- en Technologiebeleid. Halfslachtige wetenschap. Onderbenutting van vrouwelijk potentieel als existentieel probleem voor academia, Den Haag. Baar Mirjam de/Schurman, Anna Maria van (2007): Digitaal Vrouwenlexicon van Nederland, http:/www.inghist.nl/Onderzoek/Projecten/ DVN/lemmata/data/Schurman, Anna Maria van, 08.03.2007.

Berkel, Klaas van (1998): Citaten uit het boek der natuur. Opstellen over Nederlandse wetenschapsgeschiedenis, Amsterdam.

Bosch, Mineke (1988): Een vrouwenhegemonie in Baarn: Professor Dr. Johanna Westerdijk, directrice van het Laboratorium Willie Commelin Scholten. In: Jaarboek voor Vrouwengeschiedenis 9: Geleerde vrouwen, Nijmegen, S. 170-190.

Bosch, Mineke (1994): Het geslacht van de wetenschap. Vrouwen en hoger onderwijs, 1878-1948. PhD. disstertation Erasmus Universiteit Rotterdam, Amsterdam. 
Bosch, Mineke (1997): Kies exact! In historisch perspectief: veranderende visies op meisjesonderwijs en de exacte vakken, 1650-1880. In: Gewina, Nr. 20, S. 184-210.

Bosch, Mineke (2002): Women in Science: A Dutch Case? In: Science in Context, 5/4, S. 484-527.

Bosch, Mineke (2005): Een onwrikbaar geloof in rechtvaardigheid. Aletta Jacobs, 1854-1929, Amsterdam.

Bosch, Mineke/Hoving, Isabel/Wekker, Gloria (1999): In het hart van de wetenschap. Naar Total E-quality en diversiteit in de universiteit. Background Study Adviesraad voor Wetenschap- en Technologiebeleid, Den Haag.

Canel, Annie/Oldenziel, Ruth/Zachmann, Karin (2000): Crossing Boundaries, Building Bridges. Comparing the History of Women Engineers, 1870s-1990s, London.

European Commission (Osborn, Mary/Rees, Teresa/Bosch, Mineke et al.) (2000): Science Policies in the European Union: Promoting Excellence through Mainstreaming Gender Equality. A Report from the ETAN Network on Women and Science, Luxembourg.

Fox Keller, Evelyn (1985): Reflections on Gender and Science, New Haven.

Freie, Marguerite J. (1948): In opvoeding en onderwijs. In: Vrouwen van Nederland 1898-1948. De vrouw tijdens de regering van Koningin Wilhelmina. Uitgegeven onder auspiciën van de stichting Tentoonstelling de Nederlandsche Vrouw 1898-1948, hg.v. Magdalena G. Schenk, Amsterdam, S. 89-107.

Jong, Frida de (1988): „Die aloude aloë toch...“ Antonia Elisabeth Korvezee (1899-1978), de eerste vrouwelijke hoogleraar aan de Technische Hogeschool Delft. In: Jaarboek voor Vrouwengeschiedenis, Nr. 9, Geleerde vrouwen, Nijmegen, S. 227-234.

Jong, Frida de (2003): Korvezee, Antonia Elisabeth (1899-1978). In: Biografisch Woordenboek van Nederland, http://www.inghist.nl/ Nieuws/Tips/Onderzoek/Projecten/BWN/lemmata/bwn5/korvezee, 05.09.2003.

Kirejzcyk, Marta (1993): Vrouwen kozen exact; studie en beroepsuitoefening rond de eeuwwisseling. In: Gewina, Nr. 16, S. 234-247.

Kolf, Marie C. van der (1950): Zeventig jaar Vrouwenstudie, Rotterdam.

Merchant, Carolyn (1980): The Death of Nature: Women, Ecology and the Scientific Revolution, San Francisco.

Phillips, Patricia (1990): The Scientific Lady. A Social History of Women's Scientific Interests: 1520-1918, London.

Rossi, Alice S. (1965): Women in Science: Why So Few? In: Science, Nr.148, S. 1196-1202. 
Schiebinger, Londa (1989): The Mind Has No Sex? Women in the Origins of Modern Science, Cambridge/London.

Stamhuis, Ida H./Offereins, Marianne I.C. (1998): De dames op de eerste rij. Een kijkje in een natuurkundig lab in de jaren dertig. In: De universiteit als modern mannenklooster, hg.v. Balen, Barbara van/Fischer, Agneta, Amsterdam, S. 45-61.

Sturkenboom, Dorothee (2004): De elektrieke kus. Over vrouwen, fysica en vriendschap in de $18^{e}$ en $19^{e}$ eeuw, Amsterdam.

Vries, Tjitte (1970): Maria Sibylla Merian. Vrouw tussen kunst en wetenschap, AO-reeks 1307, Amsterdam.

Wennerås, Christine/Wold, Agnes (1997): Nepotism and sexism in peerreview. Commentary. In: Nature, Nr. 387, S. 341-343.

Wilde, Inge de (2001): Jantina Tammes, 1871-1947. In: Biografisch Woordenboek van Nederland $V$, Instituut voor Nederlandse Geschiedenis, Den Haag, URL:http://www.inghist.nl/Onderzoek/Projecten/ BWN/lemmata/bwn5/tammes, 22.02.2007.

Hart, Joke (Hrsg.) et al. (1986): Een barst in het bolwerk. Vrouwen, Natuurwetenschappen en Techniek, Amsterdam.

Oldenziel, Ruth (1999): Making Technology Masculine. Men, Women and Modern Machines in America, 1870-1945, Amsterdam. 

Gender \& Science -

Vielfältige Perspektiven 



\title{
Nachhaltige Ingenieurausbildung
}

\author{
CHRISTINE WÄCHTER
}

Erfolgreiche Ingenieurinnenkarrieren hängen in hohem Maße von institutionellen und organisatorischen Rahmenbedingungen in Ausbildungseinrichtungen und Betrieben ab. Das Projekt „WomEng - Creating Cultures of Success for Women Engineers“ untersuchte Einflussfaktoren auf Studien- bzw. Berufsverläufe von Ingenieurinnen.

\section{Einleitung}

Der Arbeitsmarkt ist (nicht nur) im Techniksektor vertikal und horizontal stark geschlechtsspezifisch seggregiert. Sowohl auf höheren hierarchischen Ebenen als auch in historisch männlich geprägten Bereichen befinden sich Frauen in einem eklatanten Minoritätsstatus. Im Hinblick auf eine Veränderung des unbefriedigenden Status quo hin zu mehr Geschlechtergerechtigkeit greifen Appelle, die sich einseitig an „die Frauen“ richten, wie „Frauen, geht in die Technik!“, eindeutig zu kurz. Vielmehr geht es um eine nachhaltige Veränderung maskuliner Ingenieurskultur in technischen Ausbildungsinstitutionen und Arbeitswelten.

In bildungs- und arbeitsmarktpolitischen Diskussionen lässt sich immer wieder beobachten, wie das Thema „Frauen und Technik“ hinund hergeworfen wird wie eine heiße Kartoffel, tendenziell vom eigenen Verantwortungsbereich und Handlungsraum in andere gesellschaftliche Subsysteme. Zweifelsohne gilt es in allen Bereichen aktiv zu werden, um nachhaltige Ingenieurinnenkarrieren möglich zu machen, denn der lange Weg zur Ingenieurinnenkarriere ist ein steiniger und voller Stolperstein. Im Folgenden wird es um das Themenfeld Hochschulen gehen. 
Abbildung 1: Das Modell der heißen Kartoffel

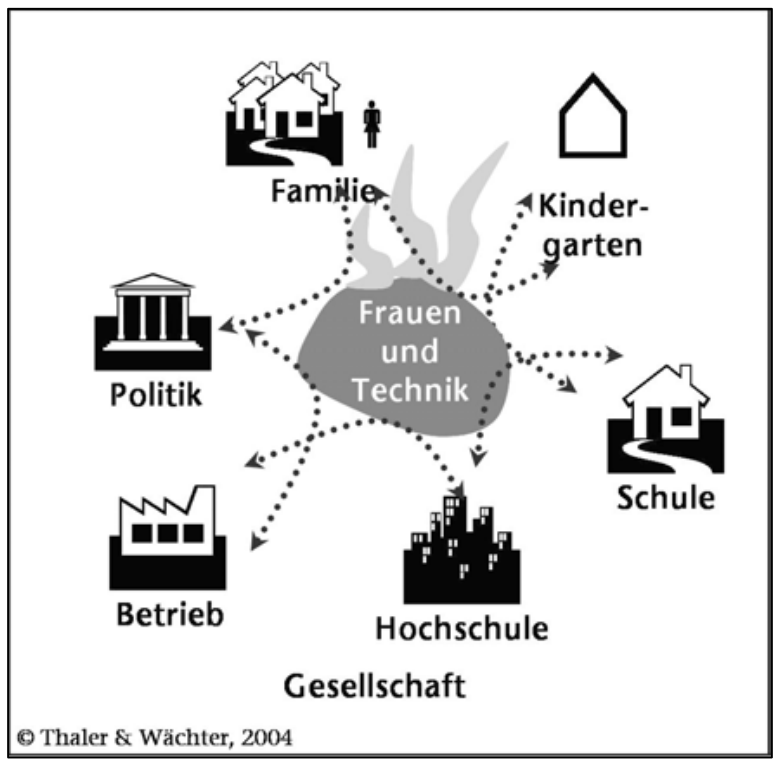

Quelle: Thaler/Wächter 2004

Skandinavische, nordamerikanische, aber auch süd- und osteuropäische Erfahrungen zeigen, dass Ingenieurinnen durchaus ,ihren Mann stellen“. Insbesondere im deutschsprachigen Raum haftet an den Ingenieur- und Technikberufen das Etikett der „Männlichkeit“ derartig fest, dass Frauen in hochqualifizierten technischen Berufen noch immer eine Minderheit darstellen (Sagebiel 2003; Wächter 2003). Dabei spielen stereotype Zuschreibungen eine nicht zu unterschätzende Rolle. Klischeehafte Vereinfachungen komplexer Sachverhalte sollen Informations- und Entscheidungsprozesse beschleunigen helfen. Reduktionistische Zuschreibungen aufgrund äußerlicher Merkmale wie Alter, Geschlecht, Ethnie werden im Alltagshandeln zumeist nicht reflektiert. Nicht bewusst gemacht wird auch der normierende Charakter solcher scheinbar objektiver Attribuierungen.

Welche Klischees werden gemeinhin mit der Ingenieurwelt assoziiert? Sie gilt als langweilig und abstrakt. Ingenieure werden als maschinenorientiert, nicht kommunikativ, nicht teamorientiert, einseitig, eindimensional, engstirnig wahrgenommen. Der geniale, aber chaotische und zerstreute Erfinderingenieur Daniel Düsentrieb oder Dilbert, ein klassisches Informatiker-Stereotyp, veranschaulichen dies in zugespitzter Form. Der „typische Ingenieur“ ist männlich, technikkompetent und ma- 
schinenorientiert. Ingenieurinnen gelten als sozial kompetent und teamorientiert, sind also eher untypisch für das Image ihres Berufsstandes (Thaler/Wächter 2006).

Die Ingenieurwelt ist geprägt durch eine maskuline Ingenieurkultur. Sie ist sexistisch. Der Ingenieur-Habitus, wie er sich in Humor, Sprache, Verhalten und Umgangsformen manifestiert, signalisiert Frauen, dass hier kein Platz für sie ist. So unterscheidet sich auch der Alltag einer Ingenieurstudentin in vielfältiger Hinsicht vom Alltag eines Ingenieurstudenten. Sie ist immer sichtbar und unter Beobachtung. Sie muss sich ständig für ihre Studienwahl rechtfertigen und verteidigen. Wenn sie „hübsch“ ist, ist sie auf „Männerfang“. Wenn sie nicht „hübsch“ ist, ist ohnehin klar, warum sie etwas Technisches studiert. Sie muss besser sein als ihre Kollegen. Ihr wird nichts zugetraut. Sie wird immer wieder in Frage gestellt und muss ihre Kompetenz immer wieder aufs Neue beweisen. Sie wird für die Sekretärin gehalten. Die Studienkollegen sind eifersüchtig und befürchten die Bevorzugung der Studentinnen. Gute Noten bekommt sie angeblich nicht, weil sie fachlich gut ist, sondern auf Grund ihres Aussehens oder „Frau-Seins“. Sie fühlt sich isoliert, nicht integriert.

Studentinnen beginnen eine technische Ausbildung mit anderen Voraussetzungen als ihre Kollegen. Sie verfügen zum Teil über weniger praktische Erfahrungen im Umgang mit Laborequipment, mit dem Computer. Sie haben häufig geringere Selbstwirksamkeitserwartung, trauen sich weniger zu und haben ein niedrigeres akademisches Selbstvertrauen. Diese unterschiedlichen Eingangsbedingungen gilt es in der Ausbildung zu berücksichtigen, denn formale Gleichbehandlung bedeutet bei ungleichen Ausgangs- und Rahmenbedingungen immer die Bevorzugung bereits privilegierter Gruppen.

Mehrere nordamerikanische Studien haben gezeigt, dass sich Ingenieurstudentinnen als Teil einer größeren „engineering community“ fühlen wollen (Goodman et al. 2002; Brainard/Carlin 1998 und 2001; Brainard et al. 1996; Adelmann 1998; Blum 2001a und 2001b). Denn nur so können sie einem Teufelskreis aus Minderheitensituation, Gefühl der Vereinzelung, niedrigem Selbstwert, niedrigem akademischen Selbstbewusstsein entkommen. Dieselben Studien haben auch gezeigt, dass Studentinnen eher unzufrieden mit dem Lehr- und Lernklima sind und dass sie stärker von schlechtem Unterricht, schlechten Lernunterlagen, schlecht aufbereiteten Lehrinhalten betroffen sind als Studenten. Ingenieurstudentinnen steigen folglich nicht aus einer technischen Ausbildung aus, weil sie es fachlich nicht schaffen oder weil sie „zu dumm sind“, sondern weil sie das Umfeld als frustrierend und demotivierend erleben. 


\section{Das Projekt „WomEng - Creating Cultures of Success for Women Engineers"}

Erfolgreiche Karrieren von Frauen in der Technik hängen zu einem hohen Maß von institutionellen und organisatorischen Rahmenbedingungen in Ausbildungseinrichtungen und Betrieben ab. Das Projekt „WomEng - Creating Cultures of Success for Women Engineers“ im 5. EU-Rahmenprogramm untersuchte von 2003 bis 2005 in sieben europäischen Ländern (Deutschland, Finnland, Frankreich, Griechenland, Großbritannien, Österreich, Slowakei) in den beiden Bereichen „Technische Hochschulen“ und „Unternehmen“ Rahmenbedingungen für erfolgreiche Studien- bzw. Berufsverläufe von Ingenieurinnen (WomengCreating Cultures of Success: Synthesis Report 2005). Die folgenden Ausführungen beziehen sich auf den Bereich „Technische Hochschulen“.

Im Rahmen einer Fragebogenerhebung unter 1.336 Studierenden wurden 699 Studierende in den Ingenieurwissenschaften (335 Studentinnen, 364 Studenten) sowie 637 Studierende in nichttechnischen Studienrichtungen (355 Studentinnen, 282 Studenten) zum Studienalltag, zur Studienmotivation und zum Studienabbruch befragt. Darüber hinaus wurden in Ergänzung zu teilnehmenden Beobachtungen und WebsiteAnalysen insgesamt 21 geschlechterhomogene Fokusgruppen mit Ingenieurstudenten und Ingenieurstudentinnen sowie 108 qualitative Interviews mit Professorinnen und Professoren, Studiendekaninnen und Studiendekanen, Gleichstellungsbeauftragten und mit erfolgreichen sowie aus- bzw. umgestiegenen Studentinnen geführt. Neben Aspekten der Studienwahl und Studienmotivation wurden interne und externe Faktoren analysiert, die das Ausscheiden verhindern und „Cultures of Success“" unterstützen (vgl. Thaler/Wächter 2005).

\section{Ergebnisse zum Studienabbruch}

Am schwierigsten ist die Studieneingangsphase. Unter anderem müssen die Studierenden erst lernen, sich zu organisieren, sich zurechtzufinden, mit den neuen Freiheiten zurechtzukommen und „lernen zu lernen“. Den Angaben unserer Interviewpartnerinnen und Interviewpartner entsprechend sind die ersten beiden Jahre die kritischen. Gerade die in den ersten vier Semestern unterrichteten wichtigen Grundlagenfächer sind besonders ,trocken“.

Etwas mehr als ein Viertel der Ingenieurstudierenden berichten von „Knock-out-Prüfungen“ und $60 \%$ aller Studierenden denken, dass diese 
„Knock-out-Prüfungen“ zu Studienabbruch führen. $71 \%$ der Ingenieurstudierenden denken, dass falsche Erwartungen vom Studiengang ein Hauptgrund für Studienabbruch sind. $79 \%$ der Ingenieurstudierenden denken, dass schlechte Prüfungsergebnisse der Hauptgrund für Studienabbrüche in ihrem Studiengang sind. $70 \%$ der Studierenden kennen mindestens eine Studienkollegin/einen Studienkollegen, die/der das Ingenieurstudium abgebrochen hat. Ein Drittel der Ingenieurstudierenden hat mindestens schon einmal ans Aufhören gedacht.

In den meisten Ländern ist bei den Gedanken an Studienabbruch der Anteil der Frauen größer als jener der Männer. Österreich ist Spitzenreiter beim Frauenanteil: 47 \% der Ingenieurstudentinnen und 29 \% der Ingenieurstudenten dachten zumindest einmal während ihres Studiums daran, das Studium aufzugeben (Thaler 2005).

Welche Gründe sind für diese Abbruchgedanken relevant? Mehr als $10 \%$ der befragten Ingenieurstudierenden haben folgende vier Gründe genannt: große Arbeitsbelastung, andere Erwartungen an das Studium, schlechte Prüfungsleistungen, Überlegungen das Fach zu wechseln. Es kommen auch interessante Länderunterschiede bei den Top-3-Gründen an Studienabbruch zu denken zu Tage. Studierende in Österreich und Deutschland nennen eher „Große Arbeitsbelastung“, für Studierende in Frankreich und Großbritannien sind „Andere Erwartungen an das Studium“ relevanter und „Schlechte Prüfungsleistungen“ lösen bei Studierenden in Deutschland und in der Slowakei Gedanken an Studienabbruch aus.

Betrachtet man die Antworten aus der Geschlechterperspektive, so sind die drei Hauptgründe von Ingenieurstudentinnen an Studienabbruch zu denken: Sich nicht Wohlfühlen, Atmosphäre im Institut/ Studiengang, Andere Erwartungen an das Studium. Die drei Hauptgründe von Ingenieurstudenten an Studienabbruch zu denken sind: Fehlende Lernstrategien, Ablehnung des Studienfachs, Finanzielle Gründe.

Ergebnisse zum Thema Studium aus dem WomEng Projekt haben gezeigt, dass mehr als ein Drittel der Ingenieurstudierenden $38 \%$ der Ingenieurstudentinnen, $35 \%$ der Ingenieurstudenten) mehr nichttechnische Fächer möchte. Mehr als ein Viertel der Nicht-Ingenieurstudierenden geben an, sie hätten ein Ingenieurstudium gewählt, wenn es mehr human- und sozialwissenschaftliche Anteile hätte. Mehr als die Hälfte aller Studierenden möchte mehr Kooperation an der Fakultät, im Studiengang. Mehr als ein Drittel der Ingenieurstudierenden, und hier mehr Frauen als Männer, möchte mehr weibliche Lehrende. Und $55 \%$ der Ingenieurstudenten im Vergleich zu 33 \% der Ingenieurstudentinnen wünschen sich mehr weibliche Studierende. 


\section{Community Building und Networking}

Um der Gefahr der Isolation und Ausgrenzung, die für Minderheiten größer ist, entgegenzuwirken, wurden eine Reihe von Maßnahmen vorgeschlagen, die durch „Community Building“ und „Networking“ ein Gefühl des Dazugehörens und Willkommenseins unterstützen und verstärken können (Wächter 2005a). Dazu zählen Begrüßungsveranstaltungen für Studentinnen, um den Kulturschock beim Einstieg in den universitären Alltag zu mildern, aber auch die Abhaltung von Frauentutorien und die Installierung von (Peer-)Mentoring Programmen.

Begleitmaßnahmen während des Semesters, wie Exkursionen, Gastvorträge, „social events“, aber auch „Karrieregespräche“, die Informationen über Berufsbilder, Berufsalltag, Berufslaufbahnen von Ingenieurinnen vermitteln, sowie Skills Workshops (z.B. Bewerbungstraining) berücksichtigen die vielfältigen Bedürfnisse von Studentinnen und können Studienabbrüchen vorbeugen. Solche Maßnahmen sind insbesondere in den ersten Semestern wichtig, da hier die „Drop out-Gefahr“ am höchsten ist (vgl. Wächter 2005b).

Außerdem wichtig sind niederschwellige Ansprechpersonen und Kooperationspartnerinnen und Kooperationspartner. Dazu gehören nicht nur die Professoren und Professorinnen, sondern auch die Sekretärinnen und Sekretäre sowie das Verwaltungspersonal, die Bibliotheksbediensteten, das Labor- und Werkstätten-Personal, die Assistenten und Assistentinnen, studentische Hilfskräfte und Ansprechpersonen auf allen Ebenen (Studierendenvertretung, Frauenbeauftragte, Studiendekan/Studiendekanin etc.). Die Schaffung von Frauenräumen, wie z.B. ein Frauencafé oder einen Frauen-Computer-Raum, bietet räumlich und zeitlich abgegrenzte Möglichkeiten des Austausches und Lernens abseits des männerdominierten Alltags.

Partnerprogramme mit Betrieben können über Praktika, Projekt- und Diplomarbeiten Firmenkontakte erleichtern und Netzwerke zu Ingenieurinnen, welchen eine wichtige Vorbildfunktion zukommt, unterstützen. 


\section{Bessere Studienbedingungen schaffen}

Eine Reihe von Vorschlägen zielt auf die Schaffung besserer Studienbedingungen ab. Da die ersten zwei Studienjahre in Bezug auf einen Studienabbruch besonders kritisch sind, sind die ersten Semester als Handlungsräume für Maßnahmen außerordentlich wichtig. Dazu wird vorgeschlagen, im ersten Studienjahr keine „Killer-Kurse“ und „Knock-outPrüfungen“ zu veranstalten. Hingegen wird ein „Common Year“ angeregt, also ein gemeinsames erstes Jahr für alle Studierenden, wie es z.B. an der Montan-Universität Leoben in Österreich erfolgreich praktiziert wird.

Interdisziplinäre Technikausbildung ist ein wichtiger Faktor für nachhaltige Ingenieurinnenkarrieren (Wächter 2004 und 2005b). Interdisziplinäre Technikausbildung fokussiert sowohl auf Inhalte als auch auf Methoden. Es gilt, Ingenieurstudien mit „,nichttechnischen“ Komponenten anzureichern, wie soziale und kulturwissenschaftliche, volksund betriebswirtschaftliche, ökologische Aspekte und der Auseinandersetzung mit Technikfolgen und damit mehr Studentinnen anzusprechen. Und nebenbei: nicht nur Studentinnen, sondern auch so genannte ,nontypical males“, also junge Männer, die nicht aufgrund eines ausschließlich auf die Technik ausgerichteten Interesses ein Ingenieurstudium beginnen.

Die Integration von Schlüsselqualifikationen (soziale Kompetenz, Konfliktmanagement, kommunikative Fähigkeiten etc.) als explizite Lernziele der Fachveranstaltungen führt auch dazu, dass diese als Berufsqualifikation erkannt und ernst genommen werden.

Mehr weibliche Lehrende in naturwissenschaftlich-technischen Fächern haben Vorbildfunktion und dadurch positive Wirkung für Studentinnen innerhalb der Hochschule sowie auf Studieninteressentinnen bzw. -bewerberinnen im Vorfeld der Hochschulausbildung selbst.

Es ist ferner wichtig, bereits zu Beginn des Studiums eine Verbindung zwischen theoretischen Kenntnissen und praktischen Erfahrungen herzustellen. Direktes Umsetzen theoretischer Kenntnisse wirkt sich durch frühzeitigen Bezug zur späteren ingenieurmäßigen Tätigkeit motivierend auf die Studierenden aus.

Zur Verbesserung der Studienbedingungen trägt auch eine verbesserte Didaktik bei. Innovative Lehr- und Lernformen wie problemorientiertes, fallbasiertes Lernen, interdisziplinäres Team Teaching, kleine Lerngruppen, kooperativ statt kompetitiv, zeitweise monoedukative Einheiten sind klassischem Frontalunterricht - wie er z.B. noch immer weitgehend in Mathematik-Vorlesungen praktiziert wird - vorzuziehen. Denn diese Vorlesungen weisen zu wenig Bezug zum eigentlichen Ingenieur- 
fach auf und sind häufig demotivierend und frustrierend. Darüber hinaus ist es wichtig, Lehrende und Studierende für das Gender-Thema zu sensibilisieren und regelmäßig und verpflichtend weiterzubilden.

An dieser Stelle soll die Wichtigkeit geschlechtergerechter Sprache gerade auch im Technikkontext betont werden. Im Alltag werden gemeinhin weibliche und männliche Formen rollenstereotyp verwendet, d.h. wir lesen und hören von Ingenieuren und Sekretärinnen, von Ärzten und Krankenschwestern, von Universitätsprofessoren und Kindergärtnerinnen, Systemadministratoren und Reinigungsfrauen. Wie verfestigt unsere Denk- und Sprachstrukturen durch diese Praxis sind, wird durch das Experiment einer Rollenumkehr sichtbar. Sprechen wir von Ingenieurinnen und Sekretären, Ärztinnen und Krankenpflegern, Universitätsprofessorinnen und Kindergärtnern, Systemadministratorinnen und Reinigungsmännern, so scheint die Wirklichkeit auf den Kopf gestellt. Sprache bildet Wirklichkeit ab, sie gestaltet sie aber auch. Nicht nur im Deutschen ist das Männliche die Norm. „Das generische Maskulinum schließt Frauen aus und macht sie in Sprache und Schrift unsichtbar" (Metz-Göckel/Kamphans 2002: 3). Gerade im Technikkontext ist es aber wichtig, Frauen nicht „mitzumeinen“, sondern sichtbar zu machen.

Als Denkanstoß zum Thema „Sprachverhalten“ sei hier ein kurzer Dialog zwischen einem Germanisten und einer Ingenieurin wiedergegeben:

Er: „Du bist also Ingenieur?“

Sie: ,IngenieurIN!“‘

Er: „Ist doch egal! Oder erschöpft sich eure Emanzipation in Formalismen?"

Sie: „Musst du ja wissen als Germanistin!“

\section{Schlussfolgerungen}

Eine Reihe von Aktivitäten in den letzten 25 Jahren hat dazu geführt, dass - im Gegensatz zu rückläufigen Zahlen bei den Studenten - kontinuierlich mehr Frauen ein Ingenieurstudium beginnen und abschließen. Mindestens genauso wichtig und notwendig wie Projekte zur Motivation und Information von Mädchen und jungen Frauen sind Maßnahmen zur Veränderung und Verbesserung der Ausbildungsinhalte sowie des Lehr-, Lern- und Arbeitsklimas (Thaler 2006). Denn erfolgreiche Karrieren von Frauen in der Technik hängen zu einem hohen Ausmaß von den entsprechenden institutionellen und organisatorischen Rahmenbedingungen in den Ausbildungseinrichtungen und Betrieben ab. 
Die Maßnahmen müssen daher auf mehreren Ebenen ansetzen. Zum einen gilt es, an den Ausbildungseinrichtungen nach innen gerichtete frauenspezifische bzw. strukturelle Maßnahmen zu implementieren. Dabei stehen die Stärkung der technisch-fachlichen Identität der Studentinnen und deren Unterstützung im Alltag in einem männlich geprägten Ausbildungsfeld im Zentrum. Zum anderen müssen aber auch Änderungen und Verbesserungen in den Studienplänen sowie methodische und didaktische Weiterentwicklungen in Angriff genommen werden.

Es gilt, technische, soziale, volks- und betriebswirtschaftliche Aspekte genauso in die Studieninhalte einzubeziehen wie Technikfolgen und ökologische Komponenten.

\section{Literatur}

Adelman, Clifford (1998): Women and Men of the Engineering Path: A Model for Analyses of Undergraduate Careers, U.S. Department of Education and The National Institute for Science Education, Washington, DC.

Blum, Lenore (2001a): Transforming the Culture of Computing at Carnegie Melon, http://www2.cs.cmu.edu/ lblum/PAPERS/TransformingTheCulture.pdf, 21.3.2006.

Blum, Lenore (2001b): Women in Computer Science: The Carnegie Mellon Experience, http://www2.cs.cmu.edu/ lblum/PAPERS/women_in_ computer_science.pdf, 21.3.2006.

Brainard, Susanne. G./Laurich-McIntyre, Suzie/Carlin, Linda. (1996):

Retaining Women in Science and Engineering. In: Journal of Women and Minorities in Science and Engineering, 2 (4), S. $255-$ 267.

Brainard, Susanne G./Carlin, Linda. (1998): A Six-Year Longitudinal Study of Undergraduate Women in Engineering and Science. In: Journal of Engineering Education, 87 (4), S. 369-375.

Brainard, Susanne G./Carlin, Linda (2001): A Six-Year Longitudinal Study of Undergraduate Women in Engineering and Science. In: The Gender and Science Reader, hg.v. Muriel Lederman/Ingrid Bartsch, London/New York, S. 25-37.

Goodman, Irene et al. (2002): Final Report of the Women's Experiences in College Engineering (WECE) Project, Cambridge, http://www. grginc.com/WECE_FINAL_REPORT.pdf, 20.11.2002.

Metz-Göckel, Sigrid/Kamphans, Marion (2002): Info-Papier No 3: Zum geschlechterbewussten Sprachgebrauch. BMBF-Projekt Neue Medien in der Bildung - Förderbereich Hochschule, Dortmund, di- 
meb.informatik.unibremen.de/documents/projekt.gender.Infopapier_ No3a.pdf, 20.12.2006.

Sagebiel, Felizitas (2003): Masculinity cultures in engineering departments in institutions of higher education and perspective for social change. In: Proceedings of the $11^{\text {th }}$ International GASAT Conference, hg.v. Jaya Naugah et al., Mauritius, S. 104-113.

Thaler, Anita (2005): Influence of Gender and Country-specific Differences on Success in Engineering Education. In: Proceedings of the Fourth European Conference on Gender Equality in Higher Education, 8.-3. 9. 2005, Oxford Brookes University, Oxford (CD-Rom).

Thaler, Anita/Wächter Christine (Hg.) (2005): Conference Proceedings of the International Conference. Creating Cultures of Success for Women Engineers, 6.-8.10.2005, Leibnitz/Graz.

Thaler, Anita (2006): Berufsziel Technikerin? München/Wien.

Thaler, Anita/Wächter, Christine (2006): Nachhaltige Ingenieurinnenkarrieren. In: Wissenschaft und Nachhaltigkeit, Forschungstag 2005. Fakultät für interdisziplinäre Forschung und Fortbildung (IFF) an der Universität Klagenfurt, Klagenfurter Beiträge zur Technikdiskussion, Heft 113, hg.v. Wilhelm Berger/Robert Lauritsch, Klagenfurt, S. 56-72.

Wächter, Christine (2003): Technik-Bildung und Geschlecht, München/Wien.

Wächter, Christine (2004): Gender-Inclusive interdisciplinary engineering education - reaching for the stars? In: Kritische Bildung? Zugänge und Vorgänge, hg.v. Werner Lenz/Annette Sprung, Münster.

Wächter, Christine (2005a): Success and Non-Persistence in Engineering Education. In: Conference Proceedings of the International Conference. Creating Cultures of Success for Women Engineers, 6.-8. 10. 2005, Leibnitz/Graz, hg.v. Anita Thaler/Christine Wächter, Graz; S. 51-61.

Wächter, Christine (2005b): Innovative Ansätze interdisziplinärer Technik-Bildung in Europa. In: Bildung in Europa: Entwicklungsstand und Perspektiven, Schriften zum Bildungsrecht und zur Bildungspolitik, Bd. 9, hg.v. Manfred Prisching/Werner Lenz/Werner Hauser, Wien, S. 141-154.

Womeng - Creating Cultures of Success: Synthesis Report (2005): http://womeng.net/intro.htm. 


\section{Ten Keys to Involve More Women in Academic Computing}

ALLAN FISHER UND JANE MARGOLIS

This note is a brief distillation of ten ideas the authors have found, in their own experience and in discussions with dozens of other universities, to be pivotal in increasing the participation of women in computing programs. The goal has been to provide a list that can serve both as a starting point and as a set of reminders to practitioners engaged in that endeavour.

\section{Introduction}

In January of 2002, MIT Press published our book Unlocking the Clubhouse: Women in Computing. The book detailed our findings and experiences with regard to gender issues in the undergraduate computer science program at Carnegie Mellon University, one of the leading academic centers of computing in the United States.

From 1995 to 1999 , we interviewed both men and women as they made their ways into and out of the program, and meanwhile worked on means of increasing the participation of women students. From the fall of 1995 to the fall of 2000 , the percentage of women among entering students rose for $7 \%$ to $42 \%$, and persistence toward graduation also improved. Since the publication of Unlocking the Clubhouse, we have had the opportunity to visit dozens of universities, mostly in the United States but also a number in other countries, to share our experiences and learn about local conditions. The details of what happened at Carnegie Mellon are reported in the book; this note synthesizes our Carnegie Mellon experiences with our subsequent learning from colleagues at many disparate universities, and attempts to distil them into a concise set of key reminders for those seeking to involve more women in their computing programs. 


\section{Understand Your System and Know Your Numbers}

While lessons from other settings and other „diversity projects“ can be instructive, it is important to understand your local situation well enough to customize a general set of strategies. The critical question is how the strategies and results from successful initiatives in other places apply to your own institution. The management truism that ,you can't improve what you don't measure" applies here. Where is the bottleneck in your department? Is it in admissions? Is it in retention? What are the retention rates of women in computer science? What have the trends been? When are people being lost? How do the numbers of women students and faculty members in your department compare to other technical departments in your institution? What is the culture of your department? How do women experience the department?

Local information, especially in the form of hard numbers, is critical to community engagement. While information about the gender-gap from other places can be imported, especially when you have a „convinced audience", there is nothing like shining a light in your dusty corners to make an institutional community take notice.

\section{Build a Powerful Team}

A significant commitment of time, energy and resources - not just blessing and encouragement - by organizational leaders seems to be essential for success. Gender investigations and interventions too often are led by „outsiders“, then marginalized and not taken seriously. At best, they often become one more report that will just sit on the shelf.

Nonetheless, the involvement of outsiders is important. Changing the dynamics of the gender-gap in computer science requires expertise from multiple disciplines (computer science, women's studies, psychology, education, history of science, anthropology, sociology, etc.). Each has a key to the puzzle that the others lack.

The end-game of building a team is the engagement of the community at large. Only through cultural change and buy-in does a transition become permanent, outlasting its original champions. Finding and facilitating „virtuous cycles“ - for example, involving more women increases faculty interest, which improves programs, which attracts more women - is an important principle. 


\section{Listen to Students}

Listening to student (and faculty) experiences can identify trouble spots in a department. But to learn about those experiences, a process that allows you to hear more than the „party line“ and more than what is „safe“ to talk about is needed. Interviewees must be able to speak confidentially, and to talk about topics not commonly discussed in computer science culture.

This process calls for some expertise with data collection methods to avoid biasing your results. For example, open-ended questions can be critical to this process because they encourage interviewees to describe and shape their own accounts of their experiences (such as „Can you tell me the story of you and computers?" or „Can you tell me about your decision to major in computer science?") rather than choosing among preselected generic answers. On the other hand, be open to using multiple forms of data gathering techniques, including survey questionnaires. Different people listen to different types of data. The key considerations are that students' own perspectives are being heard and that the process you use does not bias what you hear.

It is also important to think longitudinally. It is simplistic to think of the gender-gap in computer science in terms of a static set of influences. Rather, we must consider a web of influences and a sequence of turning points, at each of which a different set of factors may be critical. These webs of influence are most apparent over time.

\section{Focus on the Bottlenecks}

In the 1990s, Carnegie Mellon's School of Computer Science was losing women in two main ways: at admission time, where all three of application, acceptance and matriculation rates were lower for women than for men; and in the early years of the curriculum, where negative experiences and a sense of ,lack of fit" created disproportionate attrition among women. In other settings we have seen, introductory courses, processes for choosing one's major, and „weed-out“ courses have posed bottlenecks. It is critical to monitor such bottlenecks over time, and to focus interventions there. Keep in mind that eliminating a bottleneck often will involve multiple dimensions of policy, process and culture. Some key heuristics for addressing institutional bottlenecks are to

- Use appropriate recruiting and selection practices;

- Provide multiple pathways for students with differing levels of experience; 
- Mentor all students;

- Foster a high-quality and positive learning environment;

- Create and communicate a culture that supports and celebrates multiple approaches to the study of computer science.

\section{Catalyze and Support Women's Community}

A technical/professional group for women students, such as Carnegie Mellon's Women@SCS and Berkeley's WICSE, plays several important roles. Perhaps foremost, it provides an environment for women to experience being female computer scientists together with others, without feeling the need to ,learn to speak boy“ (as eloquently phrased by Anita Borg) in order to be in the field. In this vein, it provides a venue both for professional development experiences and for mutual support. The most successful instances of such groups seem to combine substantial student leadership with ongoing faculty support.

Beyond its direct impact on its membership, a women's group amplifies the visibility and voice of women in the larger community. At Carnegie Mellon, the women's group has developed representation on standing committees, has organized events for the entire community, and has developed a variety of recruiting and outreach activities - even assisting in the creation of women's groups at other institutions.

\section{Broaden the Culture}

Most computer science faculty think of Computer Science as a dynamic, multi-disciplinary field that combines aspects of mathematics, engineering and science and has application in nearly every field of human endeavour. However, many prospective students, including some of the most enthusiastic, inherit from secondary school and society a narrow notion of Computer Science as focused on computers and on coding. Addressing this ongoing legacy is a key challenge for the computing community.

Further, the introductory sequences of traditional curricula often reinforce narrow images of the field, by focusing primarily on equipping students with the programming tools they will use in later, more diverse courses. Carnegie Mellon's response to this issue has included the addition of an „,immigration course“ introducing new students to the breadth of the field. Other institutions have developed introductory courses that use integrative projects that focus on principles over programming, or 
that link Computer Science to applications, to help to broaden students' vision.

Another critical cultural notion is the image of a successful Computer Science student. Again, for many entering students as well as for many faculties, one standard profile of interests and work habits may dominate the culture. It is difficult but important to sort through the ways in which different orientations and work styles serve the institution's goals, and to support multiple approaches that work well. For example, Carnegie Mellon's admissions processes have been adapted to give weight to measures of leadership and community engagement in addition to sheer technical virtuosity.

\section{Reach Out to the Feeder Community}

Teachers, counsellors and students in secondary schools often have a very narrow view of the nature of computing and computing professions. Even where computer science programs exist, they typically focus on technical details of programming rather than the broader theory and application of computing.

Find opportunities to reach out to secondary school educators and administrators in your recruiting area, providing them with a broader picture of computer science, outreach programs, mentoring, research opportunities, and a professional community for CS teachers. They will respond by doing a better job of recruiting students for their courses, and by sending their students to you. In particular, their awareness that you are interested in promoting the involvement of women will work in your favour.

\section{Keep a Close Watch on the Student Experience}

In most academic settings, especially in large institutions, key interactions with students are factored across multiple organizations: admissions, academics, student affairs, housing, career counselling, etc. Each of these areas presents opportunities to foster or weaken a student's affiliation with a discipline. It is invaluable to work cross-functionally: both to provide students with appropriate and positive experiences, and to prevent blind spots that can miss such opportunities, or worse, create bad experiences that drive students away. 
For example, while Carnegie Mellon's reputation and recruiting power played a key role in its rapid increase in the involvement of women in computing, we believe that the university's culture of working across organizational boundaries was also an essential factor. At various times, we were able to work closely with admissions staff, other colleges, the student affairs office, and others to tailor programs to the needs of computing students, and to close up an assortment of ,cracks“ through which students might otherwise have slipped.

\section{Adapt to Changing Times}

Change is a constant, especially in a field like computing, with its rapid technological change and dynamic business cycle. Today, a new generation has grown up with the Internet and all it implies. A bit more than a decade ago we saw the first inklings of the Internet boom, and now we have been through boom, bust, and consolidation. At this writing, perhaps the key human resource challenge to the discipline of computing in the developed countries is the public perception that ,all the computing jobs are going offshore" to the developing world.

Just as the external environment changes, communities change. For example, Lenore Blum and Carol Frieze have observed a shift in the Carnegie Mellon computer science student culture, in which both men and women are likely to take a broad and connected view of the field, and in which the traditional gender stereotypes of computing are largely defused. In light of internal and external changes, it is necessary to revisit your assumptions regularly in order to adapt to new sources of challenge and advantage.

\section{Remember the First Law of Educational Diversity}

For a student who is a member of a small minority, particularly one labouring under negative stereotypes, seemingly small and sometimes unintended slights often are magnified. Cumulatively, they chip away at a student's confidence. This, in turn, often leads to a loss of interest in the discipline.

The ubiquity of these effects is tantamount to the First Law of Educational Diversity: in a situation with in-groups and out-groups, ,everything bad happens worse" for the members of the out-groups. Because of doubts about fit, comparisons with members of the in-groups, and the 
feedback between confidence and interest, bumps in the road - poor teaching, lack of advising, weed-out experiences, etc. - disproportionately create disaffection and attrition among the out-groups. Note that a corollary of this observation is that many effective interventions in favour of diversity are good for all students.

\section{Acknowledgements}

These observations have benefited from many discussions with our colleagues at Carnegie Mellon, the University of California at Los Angeles, and the National Center for Women in Information Technology (NCWIT) at the University of Colorado, Boulder. An earlier version of this note was presented at a meeting of NCWIT, and published on NCWIT's website at www.ncwit.org.

\section{References}

Carnegie Mellon University (2007): Women@SCS.

Fisher, Allan/Margolis, Jane (2002): Unlocking the Clubhouse: Women in Computing, MIT Press, Pittsburgh.

University of California Berkeley Women in Computer Science and Engineering, http://inst.eecs.berkeley.edu/ wicse/. 



\section{Informatik und Geschlechtergerechtigkeit in Deutschland - Annäherungen}

BRITTA SCHINZEL

Der folgende Text befasst sich einerseits mit Kulturvergleichen mit Bezug auf die Frauenbeteiligung im Informatik-Studium, andererseits mit Vorschlägen zur Veränderung dieses Studiums in Deutschland, um mehr Frauen für ein solches Studium zu motivieren und sie auch im Studium zu halten. Es ist eine traurige Tatsache, dass die Frauenbeteiligung in naturwissenschaftlich-technischen Studiengängen kaum irgendwo auf der Welt (nur in Japan) geringer ist als in deutschsprachigen Ländern. Die Gründe sind vielfältig und haben auch mit dem zweiten Weltkrieg zu tun, mit der Verjagung der weiblichen jüdischen Intelligenz aus Deutschland und dem Reflex der Verlegung der Kindererziehung in die Familie nach 1945. Doch ist diese Erklärung keinesfalls ausreichend. In den nordwestlichen früh industrialisierten Ländern liegt sicher mit ein Grund in der symbolischen Verbindung von Technik und Männlichkeit, die diese mitschleppen seit der Erfindung der rationalen Methode für die (Natur-)Wissenschaften, welche von Beginn an in der Renaissance zur Ausbeutung und technischen Verwertung der Natur bestimmt war. Im Folgenden werden vor allem die Gründe für Unterschiede diskutiert, auch um Hinweise zur Verbesserung der deutschen Situation zu erhalten.

\section{Einleitung und kulturelle Unterschiede der Informatik-Frauenbeteiligung}

30-40 Jahre nach der Einführung von Informatikkursen an der Universität unterscheiden sich sowohl die Teilnahme von Frauen als auch die Entwicklung der Einschreibungszahlen im Hinblick auf verschiedene Kontinente, Länder, Nationen, Kulturen, Religionen und Sprachen. Dies trifft sogar für Europa zu: im Vergleich mit den romanischen, slawi- 
schen und anderen europäischen Ländern ist die Teilnahme der Frauen in deutschsprachigen Ländern, den Niederlanden, aber auch in skandinavischen Ländern und Großbritannien extrem niedrig. In vielen westlichen Ländern ist die Anzahl der Frauen in der Informatik seit 1970 um mehr als $50 \%$ zurückgegangen. Ein sich seither wieder zeigender Anstieg ist häufig auf die höhere Beteiligung durch Ausländerinnen zurückzuführen. Gleichzeitig zeichnet sich eine Partikularisierung der Frauenbeteiligung ab, die zunehmend abhängt von vielen Faktoren, wie dem Renommee der Hochschule, einem frauenfreundlichen Klima, dem Hochschultyp (Reformuniversitäten sind bei Frauen beliebter als klassische, diese aber beliebter als technische Universitäten), guten Mentorenprogrammen und einer Einbettung in Anwendungen, wie Bindestrich-Informatiken (Bio-Informatik, Medizin-Informatik, linguistische Informatik, etc.).

Hingegen ist in so genannten industriell halbentwickelten Ländern wie in Ländern Südamerikas, Namibia, in den reichen arabischen Ländern wie Kuwait, Saudi-Arabien oder den Arabischen Emiraten, in den Tigerstaaten oder in Indien, noch in allen so genannten industriellen Entwicklungsländern, wie Burundi, Iran, Ägypten und früher Irak, die Frauenbeteiligung viel höher, oft gar größer als der Anteil männlicher Studierender in Informatik und Mathematik. Besondere Beachtung verdienen die hohen Frauenzahlen in der Technik in arabischen Ländern wie Kuwait, Saudi-Arabien, aber auch Ägypten und Irak (vor dem IrakKrieg), und dies weitgehend unabhängig davon, ob der Schulunterricht und/ oder das Studium in den einzelnen Ländern geschlechtssegregiert ist (Kuwait, Saudi-Arabien) oder nicht (Ägypten, Irak), während die Segregation in deutschsprachigen Ländern zum Ausfall der geschlechtsrollentypischen Fächerwahlen führt. ${ }^{1}$

Die extrem hohe Teilnahme der Frauen im Bereich der Ingenieurwissenschaften und Informatik in den nordafrikanischen und arabischen Ländern kann auf der einen Seite mit dem horizontalen geschlechtsspezifischen Arbeitsmarkt, der geschlechtsspezifische Themen und Berufe über den geschlechtshierarchischen Arbeitsmarkt definiert und erklärt werden: Religion als höchster Prestigeträger definiert entsprechende Berufe als rein männliche Angelegenheit, wohingegen Technologie nicht geschlechtsabhängig ist. Auf der anderen Seite ist die Trennung der Geschlechter innerhalb der Erziehung offenbar von geringerer Bedeutung: In Saudi Arabien und Kuwait gibt es auf keiner Bildungsebene Koedukation. Jedoch befinden sich auch in arabischen Ländern mit Koeduka-

1 Vgl. Schinzelhttp://mod.iig.uni-freiburg.de/cms/fileadmin/publikationen /online-publikationen/Frauenanteil. Informatik.International.pdf 
tion wie Ägypten, Irak oder Tunesien sehr hohe (hälftige) Frauenanteile in naturwissenschaftlich-technischen Fächern. Für Nordwest-Europa und Amerika ist gut erforscht, dass durch Koedukation die Frauenteilnahme in Natur- und Ingenieurswissenschaften während der Pubertät vermindert wird. Es ist aber sehr wichtig zu bemerken, dass das nicht überall in den europäischen Ländern zutrifft, z.B. in Italien oder in Belgien, und so offenbar auch nicht in den arabischen Ländern!

Es zeigt sich so, dass viele von den so genannten industriell entwickelten Ländern bezüglich der Geschlechterverhältnisse verhältnismäßig „unterentwickelt“ sind, wenn es um die Aufnahme der Frauen in diese „harten“ und einflussreichen Fächer geht. Eine bemerkenswerte Beobachtung ist außerdem, dass der Frauenanteil in Naturwissenschaft und Technik innerhalb von Europa in den ehemaligen sozialistischen und romanischen Ländern höher ist als in den angelsächsischen, skandinavischen und deutschsprachigen Ländern. Innerhalb von Europa sind die Türkei, Spanien und Portugal in Bezug auf die Aufnahme der Frauen in Naturwissenschaft und Technik auf allen Stufen der Karriereleiter insgesamt führend.

Abbildung 1: Internationaler Vergleich von Frauenanteilen in technischen Fächern. Weibliche Absolventen in Prozent aller Absolventen der technischen Fächer (Mathematik, Informatik, Ingenieurwesen, Herstellung und Aufbau) des jeweiligen Landes

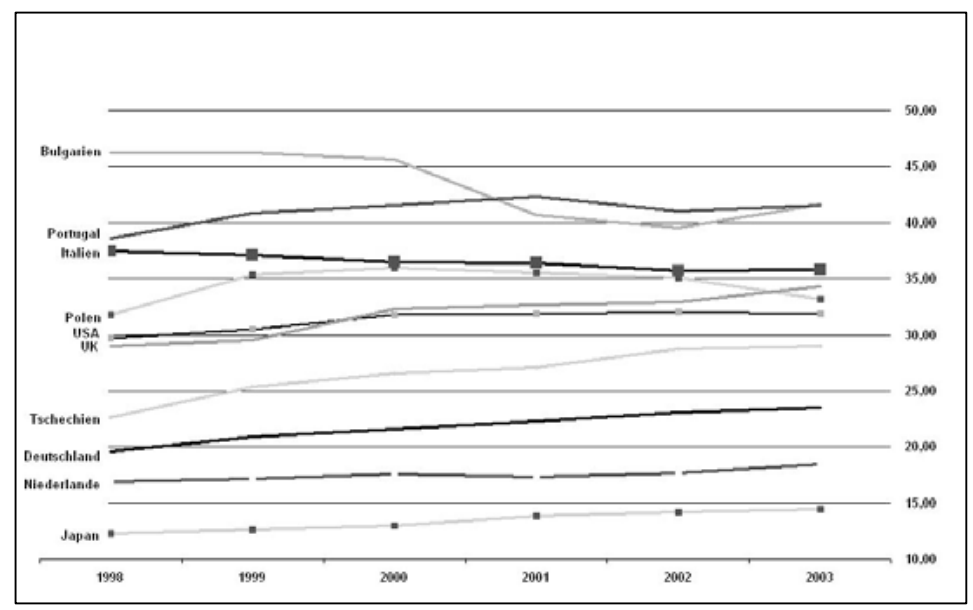

Quelle: Eurostat

Unterschiede zwischen romanischen und slawischen Ländern auf der einen Seite und skandinavischen, angelsächsischen und deutschsprachi- 
gen Ländern auf der anderen Seite können auf die spätere Industrialisierung, an deren Transportmittel, die Technik und die männlichen Geschlechtsrollentraditionen der rationalen Wissenschaften nicht mehr so unauflösbar kleben, zurückgeführt werden. Auch die unterschiedlichen Religionstraditionen können dafür von Gewicht sein. Der Zusammenhang zwischen Protestantismus und Kapitalismus könnte auch auf einen Zusammenhang zwischen Protestantismus/ Kapitalismus und die Positionierung von Geschlechtsrollen im technischen Bereich deuten. In katholischen Ländern wird das „,doing gender“ mehr an traditionelle Geschlechterkulturen geknüpft. Die Performanz des Geschlechts kann über Kleidung, unterschiedlichen Habitus, Auftritte in der Öffentlichkeit, Geschlechter-Gemeinschaften und machtvolle weibliche Familienrollen ausgeübt werden und knüpft sich weniger an Kompetenzzuschreibungen oder Berufe.

In Spanien und Portugal hat zudem der Übergang zur Demokratie in den 70ern und 80ern eine wichtige Rolle für die Veränderung der Rolle der Frau in der Gesellschaft gespielt. Das massive Eindringen der Frauen in den Ausbildungsbereich ereignete sich in einer Zeit, als es einen starken Bedarf an Arbeitskräften im technologischen Bereich gab. Auch das erklärt den Zuwachs der Anteilnahme der Frauen in technischen Studiengängen in den 80er Jahren, wobei hier wieder die spätere Industrialisierung mit eine Rolle spielt.

\section{Die Situation in Deutschland}

Die Beobachtung der Unterschiede in verschiedenen Ländern zeigt, dass es keine inhärenten, gar biologisch determinierten, sondern stark kulturell geprägte und strukturelle Gründe für die Segregation der Geschlechter in Studium und Beruf gibt.

Innerhalb unserer Kulturen werden die Werte von Gleichberechtigung der Geschlechter, Freiheit und Dekonstruktion der Geschlechtsunterschiede häufig als Argumente für die Naturalisierung der Geschlechtdifferenzen im Berufsbereich angeführt: „Frauen interessieren sich eben nicht für Technik“.

Seit der Etablierung der Informatik-Studiengänge Anfang der 70er Jahre gab es einen konstanten Anstieg der Einschreibung von Studentinnen und Studenten. Zwar war die Teilnahme der Frauen mit max. 25 \% erheblich geringer als die der Männer, dennoch galt es zunächst als offenes Studium, nicht festgelegt durch Geschlechterzuschreibungen. Während der 80er und 90er nahm die Anzahl der Frauen mit zwei Brüchen 
erheblich ab, bis zu nur mehr $7 \%$, um sich Ende der 90er bis heute wieder auf ca. $14 \%$ zu erholen.

Das folgende Diagramm zeigt deutliche Unterschiede zwischen den vom Statistischen Bundesamt angegebenen und vom BMFT (bis 2001) sowie den vom Fakultätentag Informatik (leider nur bis 2002) erhobenen Daten. Die Letzten zählen nur die „,echten“ Studierenden, die sich in Vorlesungen aufhalten und zu Prüfungen anmelden, nicht aber beispielsweise die Parkstudierenden. Sie sind daher die im Sinne der Ausbildung realistischeren Daten.

\section{Abbildung 2: Frauenanteil Informatik Studienanfängerinnen}

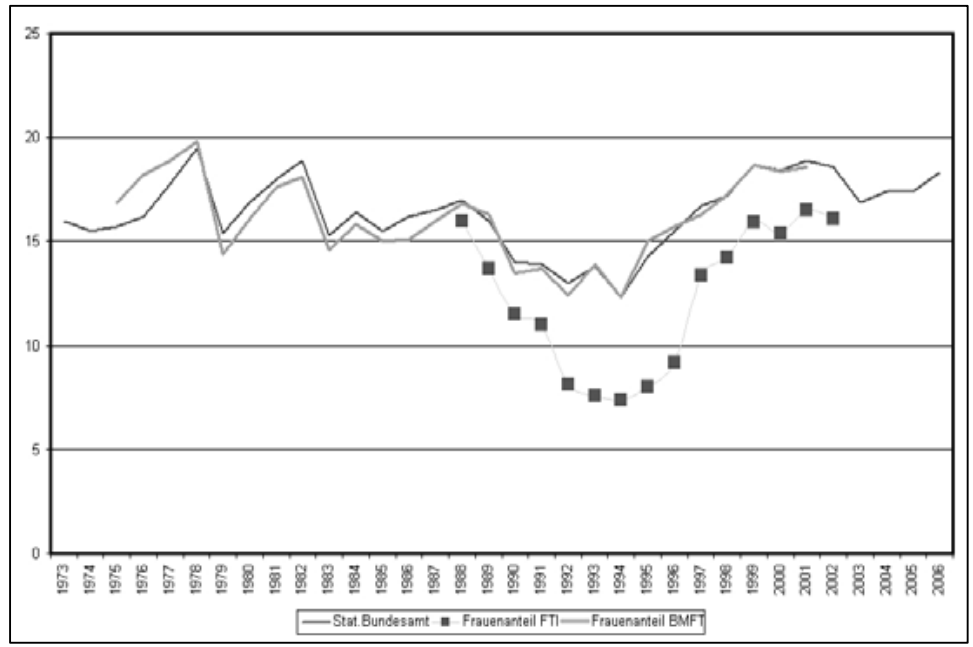

Quellen: Statistisches Bundesamt, BMBF, FTI; eigene Darstellung

Im Gegensatz dazu war die Anzahl in der ehemaligen DDR gleichmäßig auf die Geschlechter verteilt oder Frauen dominierten. 
Abbildung 3: Frauen in der Informationsverarbeitung in der ehemaligen $D D R$

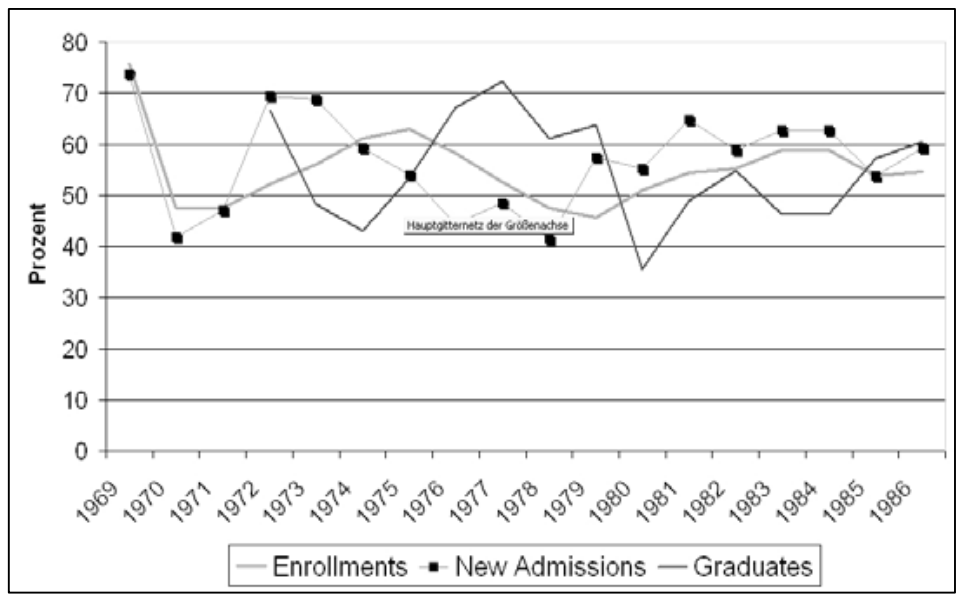

Quelle: Dolores Augustine, 1999; eigene Darstellung

Doch hatte die Wiedervereinigung auf die Frauenanteile im Fach Informatik gravierende Folgen, wie hier am Beispiel Rostocks gezeigt:

Abbildung 4: Auswirkung der Wiedervereinigung Deutschlands auf die Einschreibung von Studentinnen im Studiengang Informatik an der Technischen Universität Rostock.

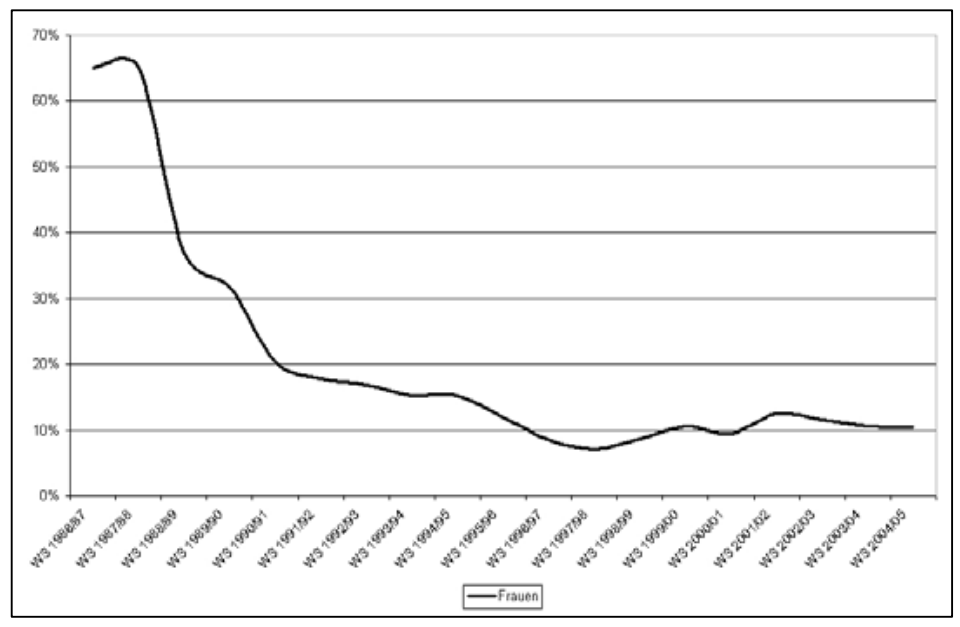

Quelle: Universität Rostock; eigene Recherche und Darstellung 
Ähnliche Entwicklungen beim Übergang zur freien Marktwirtschaft wie in den neuen deutschen Ländern ereigneten sich auch in Ungarn (nach einem Kollegen in Budapest waren im Jahr 2000 ungefähr $10 \%$ aller Informatik-Studierenden Frauen) und Tschechien, jedoch nicht in allen ehemals sozialistischen Ländern. In den baltischen Ländern ist der Frauenanteil nach wie vor sehr hoch, in Rumänien liegt er bei ca. $30 \%$, bei allerdings sehr geringen Gesamtzahlen, in Russland liegt er bei 15$20 \%$ (Cisco), während ich 1982 bei einem Aufenthalt in Leningrad an der Universität $60 \%$ Frauen in Mathematik/Informatik vorfand und an der Universität Riga gar 96 \%. Allerdings ist zu bemerken, dass die Berufsaussichten für Mathematikerinnen und Mathematiker und Ingenieurinnen und Ingenieure in der Sowjetunion insgesamt schlecht waren. Viele der Mathematikerinnen arbeiteten später als Sekretärinnen. Hoch angesehen waren dagegen die Fächer Physik und Chemie, weshalb sich dort auch viele männliche Studierenden befanden. Oft wurde auch die negative Wirkung der langen Militärzeit für die Studierwilligkeit der männlichen Studierenden beklagt, sowohl wegen des hohen Alters als auch wegen der Alkoholprobleme. In Bulgarien waren 199670 \% der Ingenieurstudierenden Frauen (Lovegrove 1991), wofür es strukturelle Erklärungen gibt: der Weg zum Studium hing von Zulassungsprüfungen $a b$, bei welchen Frauen auch in den Ingenieursfächern besser abschnitten $^{2}$. Nach 1990 wurde der weibliche Überhang als sozial unverträglich angesehen, weshalb nunmehr eine Männerquotierung eingeführt wurde, die die Notenanforderungen entsprechend der Wunschquote von $50 \%$ nach Geschlecht differenziert.

Die sehr hohe Beteiligung von Frauen in der DDR (und in den früheren sozialistischen Ländern generell) ist nur mittels eines differenzierten Komplexes von Gründen erklärbar. Zunächst ist festzuhalten, dass die Zulassungen zu den verschiedenen Studienrichtungen lange Zeit staatlich gelenkt waren, gemäß der Arbeitsmarktanforderungen und anderer Gesichtspunkte. Doch waren später während der 80er Jahre die Zugänge nahezu frei, und trotzdem war die Geschlechterverteilung in Informatik immer noch zugunsten der Frauen. In sozialistischen Ländern wurde die Gleichberechtigung der Geschlechter immer hervorgehoben. Diese angenommene Gleichberechtigung wurde von Frauen durchaus internalisiert, auch dann, wenn eine mehr oder weniger subtile Ungleichverteilung der einflussreichen Berufe und der Aufgaben in Familie und Kindererziehung durchaus existierte (Augustine 1999). Ganztagskindergärten waren für jedes Kind vorhanden und $98 \%$ aller Frauen in dem ent-

2 Ähnliches gilt übrigens für die arabischen Staaten und erklärt die hohe Frauenbeteiligung dort. 
sprechenden Alter arbeiteten. Bis in die 80er wurde der Hauptanteil der Studenten in Hinblick auf kommunistische Ideale orientiert, die sich auf die Technologien der Zukunft und Gleichberechtigung der Geschlechter, aber auch auf die Anforderungen des Arbeitsmarktes bezogen. Sicherlich zwang dieser Umstand viele Frauen im Gegensatz zu ihren Interessen, Naturwissenschaften, Mathematik und Technologie zu studieren, wie Dolores Augustine richtig beobachtet (vgl. ebd.). An den Schulen wurde der Schwerpunkt stärker auf die Natur- und Ingenieurwissenschaften gelegt als im Westen, und alle Schüler konnten Erfahrungen mit Technik und Technologien durch das obligatorische Praktikum in Firmen im Rahmen der ,polytechnischen Erziehung“ machen. Neben anderen Wissenschaftlerinnen und Wissenschaftler stellen Breckler et al. fest, dass die praktische Auseinandersetzung mit Technik besonders wichtig ist, um Interesse an ihr zu finden, Erfahrungen zu sammeln, was schließlich die Entscheidung für einen Beruf in entsprechenden Bereichen erleichtert (Breckler 1991). Diese verschiedenen Ursachen könnten erklären, warum so viele Frauen in der DDR Informatik studierten.

Aber warum nahm die Prozentzahl unmittelbar nach der Wiedervereinigung ab? Diese Entwicklung kann leicht erklärt werden: Es gab eine dezidierte Politik, Frauen vom Arbeitsmarkt abzuhalten, um diesen den „alten Bundesländern“ Deutschlands anzugleichen. Kindergärten wurden geschlossen, Politiker erklärten, dass die Arbeit der Frauen auf einen ,normalen“ Zustand (d.h. Westdeutschland) reduziert werden sollte. Zwischen 1990 und 1992 wurden die meisten Arbeitsplätze in der ehemaligen DDR ,abgewickelt", wobei viele Menschen ihre Stellen verloren. Im Bereich der Ingenieurswissenschaften erhielten Männer ihren Arbeitsplatz nach der Abwicklung meist wieder oder bekamen andere Stellen, während Frauen nur abgewickelt, d.h. endgültig entlassen wurden (Burkhard 1997). Natürlich ermutigte dieses Vorgehen Frauen nicht gerade, ein Studium der Informatik oder der Ingenieurswissenschaft aufzunehmen. Das war gerade der Effekt, der erzielt werden sollte.

Die auffällig starken kulturellen Unterschiede an Teilnahmen der Geschlechter in naturwissenschaftlich-technischen Fächern und noch einmal mehr in informatischen Studien und Berufen sind auf ein komplexes Ursachengefüge zurückzuführen, das in seiner Gesamtheit vermutlich nicht zu klären ist. Sicher jedoch ist, dass strukturelle, aber auch symbolische Faktoren in Wechselwirkung zu den Kulturunterschieden in der Beteiligung beitragen. $\mathrm{Zu}$ den strukturellen Faktoren gehören die Strukturen der Gesellschaft wie Schichten, Klassensysteme, institutionell unterstützte Familienstrukturen, Schulsysteme in all ihren Facetten, einschließlich Mono- oder Koedukation, Universitätszugangssysteme 
und Universitätsstrukturen, sowie strukturelle Berufszugangssysteme. Wie subtil solche Ein- bzw. Ausschlussmechanismen wirken, hat eine neue Arbeit beschrieben, die die Wirkung von Strukturen der Arbeitsämter in Deutschland auf die Vermittlungserfolge für Frauen in naturund ingenieurwissenschaftlichen Berufen zeigt, und das ohne dass irgendwelche Geschlechterbias auf Seiten der Vermittlerinnen und Vermittlern festzustellen waren. In die institutionellen Strukturen gehen jedoch symbolische Zuordnungen von Geschlecht und Technik mit ein, die sich dann an beispielsweise durch Software verfestigten Vorrangbeziehungen und an Regelungen wie dem Arbeitsschutz, der Arbeitszeiten, der Mobilität etc. festmachen.

Besonders interessant, jedoch schwierig zu erklären, sind die auffallenden Unterschiede innerhalb Europas. Es bestehen im Bereich der Informatik in den romanischen und slawischen Ländern wie auch in der Türkei geringere Geschlechtsunterschiede als in Großbritannien, in den skandinavischen und deutschsprachigen Ländern. Dieser Umstand lenkt die Aufmerksamkeit von strukturellen Gründen auf die symbolische Bedeutung der Beziehung von Geschlecht und Technologie. Diese Entwicklung könnte auch die Diversifikation in Bezug auf die verschiedenen Universitätstypen in Deutschland erklären.

Es scheint ein starkes Bedürfnis zu geben, „Geschlechtsunterschiede“ auf die eine oder andere Weise ,auszudrücken“. Wenn Geschlechtsunterschiede institutionell garantiert oder kulturell festgelegt sind, wie es mit Hilfe von visuellen Kennzeichen, z.B. bestimmter Kleidung, Frisur, einem Bart oder einer klaren Hierarchie in der Arbeitsaufteilung Familie zum Ausdruck gebracht werden kann, dann kann diese Darstellung der Geschlechtsunterschiede ohne eine Kennzeichnung des Geschlechts unter Aspekten von Kompetenzen erfolgen. Das gilt auch für die romanisch-katholischen und slawischen Kulturen in Europa, in denen eine stärkere Kennzeichnung des Geschlechts durch den Körper als in protestantischen Kulturen vorkommt. In den katholischen Ländern gibt es eine stärker ausgeprägte kulturelle Kennzeichnung der Geschlechter, die beiden Geschlechtern ein starkes Bewusstsein über ihre sexuelle Identität verleiht. Dieses Kulturverhalten tritt vor allem in der Interaktion zwischen Männern und Frauen außerhalb des Arbeitsplatzes auf und bestätigt ihr entsprechendes Selbstbewusstsein als Mann oder Frau. In den Ländern des Balkans, in Italien, in Spanien und Portugal existieren ausgeprägte und selbstbewusste Geschlechtskulturen, die die Rolle der Mutter für alle Generationen wie für Gruppen von Jugendlichen und deren Auftreten in der Öffentlichkeit betreffen. In Russland verleiht die 
allgemeine Auffassung, dass Männer für die Organisation ihres Alltags (wie auch dank Trunksucht oft im Beruf, weshalb entscheidende Stellen häufig doppelt besetzt sind, mit einem Mann der offiziell leitet und einer Frau, die die Arbeit macht ${ }^{3}$ ) nicht geeignet sind den Frauen ein recht ausgeprägtes Kompetenzbewusstsein und ein Bewusstsein ihrer Geschlechtsidentität, das Unterschiede jedoch nicht in Frage stellt. Es besteht daher keine Notwendigkeit für Männer und Jungen, in Mathematik, Naturwissenschaften oder Ingenieurwissenschaften hervorzutreten, um ihre männliche Identität $\mathrm{zu}$ bestätigen. Vor diesem Hintergrund können Frauen sich einfacher als intellektuell gleichwertig verstehen, auch in Bezug auf die Informatik. Sie müssen sich nicht von Ingenieurwissenschaft und Informatik distanzieren, um als „richtige“ Frauen zu gelten, wie es oft in den nordwestlichen europäischen Ländern beobachtet werden kann.

Innerhalb der protestantischen Kulturen besteht eine größere Tendenz, den Körper und Sexualität zu verstecken, und Kleidung und viele Verhaltensweisen anzugleichen. Durch die zusätzliche Auflösung geschlechtlicher Unterschiede im Gesetz und anderen institutionellen Formen kommt die Geschlechtsunterscheidung auf andere Weise zum Vorschein. Gesellschaften tendieren zur Aufrechterhaltung von den Hierarchien der Geschlechter durch Reproduzierung der Geschlechtskennzeichnung, insbesondere in hoch angesehenen Bereichen wie Informatik. Es scheint demzufolge in den zuletzt genannten Kulturen ein Bedürfnis zu geben, Differenzen individuell durch ein bewusstes Vorgehen zu äußern, wobei dieses Vorgehen symbolisch markiert wird. Als Folge daraus entwickeln sich Geschlechtsunterschiede kontextbedingt, und ihre Entstehung erweist sich als ein Prozess mit vielen Vorbedingungen, die von spezifischen Konstellationen und verschiedenen Gebieten der menschlichen Aktivität abhängen.

Daher lösen sich Geschlechtsunterschiede in bestimmten Kontexten auf, wohingegen sie in anderen erhalten oder sogar verstärkt werden können (Heintz 1998), wie es auf dem hoch angesehenen Arbeitsmarkt der Informatik in einigen Ländern der Welt der Fall ist. Obwohl das Geschlecht als ordnender Faktor der Gesellschaft seine Wichtigkeit in den nordwestlichen Ländern Europas im Allgemein zu verlieren scheint, zeigt es eine bemerkenswerte Dauerhaftigkeit im Hinblick auf die hierarchischen Strukturen. Außerdem gewinnt es eine stärkere Bedeutung auf der symbolischen Ebene mit Bezug auf die Ordnung der Inhalte, der

3 Persönliche Auskunft der Freiburger Slawistin Prof. Dr. E. Chaurè 
wissenschaftlichen Themen, und der spezifischen Berufe. Die Kontextualisierung der Geschlechtsunterschiede könnte durch die neue Eröffnung von Räumen verursacht werden, die die Definition der Geschlechtsidentität eher dem Zufall und dem Individuum überlässt. Leider wird dieser freie Raum nicht für die Konstruktion einer Identität genutzt, die Gleichberechtigung ermöglicht. Die Markierung der Kompetenz durch das Geschlecht ist gleichzeitig mit einer symbolischen geschlechtsspezifischen Kennzeichnung der Fächer verbunden, die Abgrenzungen zwischen und den sozialen Ein-/Ausschluss von Männern und Frauen erzeugt.

\section{Diversifikation der Frauenteilnahme in Deutschland}

Tracy Camp (Camp 1997) bemerkte, dass in den USA verhältnismäßig sehr viel weniger Frauen an technischen Universitäten als an ,nichttechnischen" Universitäten Informatik studieren. Dieser Trend ist seit mehr als 10 Jahren in den USA zu beobachten. Wir stellen ähnliche Phänomene seit kürzerer Zeit auch in Deutschland fest. An technischen Universitäten befinden sich weniger Informatik-Studentinnen als an traditionellen Universitäten des Humboldt'schen Typus und an diesen wieder weniger als an Reformuniversitäten, wo auch vermehrt interdisziplinäre Informatik-Studiengänge angeboten werden. Des Weiteren werden die meist technischer orientierten Fachhochschulen von Frauen insgesamt weniger für ein Informatik-Studium gewählt als die stärker theoretisch-mathematisch orientierten Universitäten.

Stärker spezialisierte Kurse weisen zunehmend signifikantere Effekte im Hinblick auf geschlechtsspezifische Unterschiede auf: je technischer definiert (d.h. benannt) desto geringer ist die Teilnahme der Frauen, und je interdisziplinärer und je stärker auf Anwendungen bezogen definiert desto höher ist der Teilnahme der Frauen (z.B. FH Furtwangen 2000/ 01: Technische Informatik 0,0 \% Frauen, Medien-Informatik 27,8 \% Frauen).

In unserer Untersuchung (Huber 2002) der Situation der Frauen im Bereich der Informationstechnik in der Industrie wie auch bezüglich der Universitätsausbildung innerhalb des Bundeslandes Baden-Württemberg zeigte sich die stärkere Diversifizierung und Kontextualisierung der Frauenbeteiligung ebenso wie in einer Untersuchung von Heintz und Nadai (Heintz 1998) in der Schweiz. Insbesondere zeichnet sich im Ausbildungsbereich der Universitäten eine starke Abhängigkeit von der 
Technikdetermination und vom Anwendungskontext ab. Je näher die (oft nur metaphorische) Bezeichnung und die Selbstdarstellung eines Studiengangs in die Nähe von Technik oder Ingenieurwesen rückt desto weniger Frauen zeigen Interesse, je mehr der interdisziplinäre, der Anwendungs- und/ oder der soziale Kontext betont wird desto mehr Frauen nehmen teil. Diese Tatsache ist weniger abhängig von den tatsächlichen Inhalten eines Studiums, denn Frauen wählen Studienrichtungen mit kontextueller Nähe zu Kommunikation und Sozialem bzw. mit Distanz zu Technik, selbst dann, wenn die Inhalte des Studiums vorwiegend technischer Natur sind (wie es für die noch wenig durch Normen geprägte, kaum mathematisch durchdrungene und somit durch technisches „Stricken“ und „Basteln“ bestimmte Medien-Informatik der Fall ist). Daher ist es notwendig, den Technikbegriff, der offenbar in unserer Kultur symbolisch stark androzentrisch aufgeladen ist, in jedem seiner Bedeutungen und Kontexte genau zu analysieren und hinsichtlich der „Genderladung“(Crutzen 2000) zu dekonstruieren. ${ }^{4}$

\section{Abbildung 5: Studierende in informatischen Fächern WS 2003/ 04}

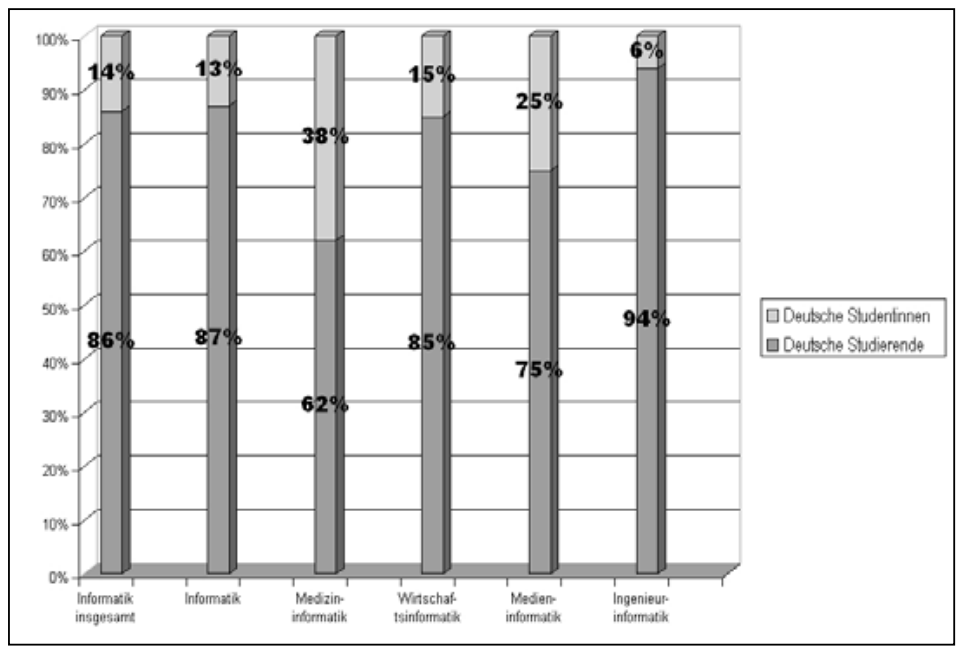

\section{Quelle: Statistisches Bundesamt; eigene Darstellung}

Die letzte Abbildung zeigt gleichzeitig, dass für das Informatik-Studium der Anteil an weiblichen Ausländerinnen höher ist als an deutschen Studentinnen. Das trifft auch für Töchter von Migrantinnen und Migranten zu, die in der folgenden Grafik jedoch nicht aufscheinen.

4 Siehe Schinzel 2001 für Engineering-Bezeichnungen in der Informatik 
Abbildung 6: Ausländische Studierende Informatik

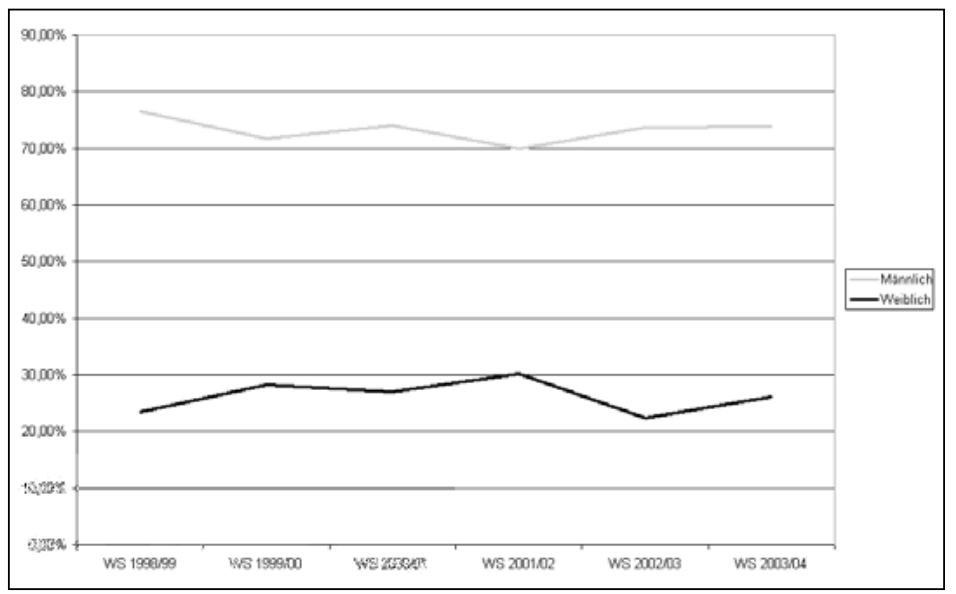

Quelle: Statistisches Bundesamt, Stand: 2004; eigene Darstellung.

\section{Maßnahmen zur Erzielung von mehr Geschlechtergerechtigkeit im Informatik-Studium}

Ein breites Feld von Ursachen für die geringe Beteiligung von Frauen und die zunehmende Verdünnung ihrer Beteiligung in Informatik und Informationstechnik in den nordwestlichen Industrieländern ist identifiziert worden. Sie reichen von der kulturellen Verankerung der Verbindung von Technik und Männlichkeit aus der Geschichte der rationalen Wissenschaften und der frühen Industrialisierung (während später industrialisierte Länder dieser Verknüpfung weniger ausgesetzt sind), über viele mit ebendieser Vorstellung beladene Transportmittel in der Sozialisation, die in unserer Kultur dazu führen, dass der Computer zu einem Stabilisationsmittel für Männlichkeit in der Pubertät geworden ist, und daraus resultierende Negativeffekte von Selbstbewusstsein und Aneignung bei Mädchen, sowie der Koedukation in Informatik und mathematisch-naturwissenschaftlichen Fächern an der Schule ${ }^{5}$ (die bei-

5 So war für die Schülerinnen aus monoedukativen Gymnasien in unserer Schülerinnenstudie Anfang der 90er Jahre (Funken 1994) Informatik das zweitleichteste und zweitinteressanteste Fach, während es für Schülerinnen aus koedukativen Gymnasien als das schwerste und uninteressanteste Fach galt. Entsprechend geringe Geschlechtsrollenorientierung hatten auch die Jungen aus monoedukativen Gymnasien. 
spielsweise in romanischen Ländern nicht zu ähnlichen Effekten führen) bis hin zum Mangel an weiblichen Vorbildern (Schinzel 2003a; Roloff 1989 u.a.).

Auch wenn die meisten dieser Gründe wegen ihrer komplexen sozialen Verankerung nicht einfach eliminiert werden können, so sind doch Veränderungen in der Ausbildung möglich, die zu spürbarer Verbesserung der Frauenbeteiligung führen können, wie an der Carnegie-Mellon Universität in Pittsburgh, USA geschehen (Blum 2001). Ich beschränke mich daher auf Maßnahmen an den Universitäten selbst bzw. von den Universitäten aus, da sonst eigene Aktivitäten abgewiegelt werden können, und das Problem nur von einem zum nächsten verschoben wird, von der Universität auf die Schule, von der Schule aufs Elternhaus, von den Eltern auf die Schule und auf den Arbeitsmarkt und von diesem wieder auf alle vorhergehenden.

Zunächst ist allerdings zu erwägen, was Geschlechtergerechtigkeit in der Informatik bedeuten kann. Gleichberechtigter Zugang in bestehende Studienstrukturen und Curricula ist selbstverständlich gegeben. $50 \%$ Frauen als Quote bei weiterhin bestehenden Strukturen kann jedoch auch keine nachhaltige Lösung sein. Vielmehr wäre auf die vielfältigen strukturellen und symbolischen Barrieren zu achten, die sich für Frauen in deutschsprachigen Ländern aufgrund ihrer Möglichkeiten zur Lebensplanung, der geschlechtsspezifischen Sozialisation mit Bezug auf Technik, der „Diversity“ unangemessenen Curricula und Lehre etc. ergeben. Es ist umso wichtiger, entsprechende Maßnahmen einzuleiten, als das Anhalten der Situation selbst verstärkenden Charakter hat: Solange eine bestimmte soziale Gruppe innerhalb der Informationstechnologie, welche Wissen und soziale Ordnungen formt, die Mehrheit stellt, werden in der Struktur und in den Anwendungen dieser Technologie dieser Gruppe entsprechende Lebens- und Wahrnehmungsweisen reflektiert. Dies hat Folgen für die Anziehungskräfte an Personengruppen und für die Veränderungen von Arbeit, Freizeit und Organisation durch diese Technologien, welche sich denn auch deutlich zeigen.

Ansatzpunkte für veränderte Studienstrukturen und Curricula sind folgende, beispielsweise in unserer „Informatik-StudentInnenstudie“ (Schinzel 1999a) identifizierten Barrieren für Frauen: Geringere Vorerfahrungen beim Programmieren, geringeres Selbstbewusstsein, eine Frauen abweisende Computerkultur und eher an Männerwünschen orientierte Curricula und Lehrformen. In unserer Studie zeigten sich signifikant weniger Studentinnen intrinsisch am Studium interessiert als Studenten (d.h. an den Inhalten Programmieren und Formales) und signifikant mehr Frauen extrinsisch am Studium interessiert (Erwartung eines 
interessanten späteren Arbeitsplatzes) als männliche Studenten. Das bedeutet aber, wenn die Sinnorientierung des Studiums anhand der Anwendungen und der Kontextualisierungen in den Vordergrund gerückt wird und die technischen und formalen Inhalte einen dienenden Charakter annehmen (ohne ihren Umfang und ihre Tiefe zu verlieren), so wird das Interesse der Studentinnen ebenfalls ein intrinsisches. So einfach lässt sich die Motivation steigern, auch die der meisten männlichen Studierenden. Bei allen Studierenden unserer Studie waren Vorlesungen, die neben Übungen und Tutoraten den meisten Raum in den bundesdeutschen Lehrplänen der Informatik beanspruchen, relativ unbeliebt. Vor allem Informatikstudentinnen sähen gerne die „Berieselung“ durch frontalen Unterricht im Hauptstudium durch bidirektionale Lehrformen wie Seminare, Projektgruppen und Praktika ersetzt, in denen sie ihre eigene Kreativität besser wahrnehmen können. Es sollte dabei immer wieder deutlich werden, dass Maßnahmen, die die Beteiligung von Frauen fördern, keineswegs Männer benachteiligen, sondern im Gegenteil die vorgeschlagenen Maßnahmen allen Studierenden zugute kommen. Das Studium sollte so gestaltet werden, dass beide Geschlechter mit gleichen Anfangsbedingungen eintreten, dass beide gleichermaßen ihre Interessen befriedigt sehen, ihre Motivation nicht verlieren und beide gleich günstige Lern-, Studien- und Prüfungsbedingungen vorfinden. Überdies sollte es zu Fähigkeiten verhelfen, die sinnvolle und gute Softwareentwicklung begünstigen. Diese Anforderungen betreffen nicht nur das Curriculum und die Inhalte des Studiums selbst, sondern auch die gesamte Vermittlungskultur, also die Didaktik, die Lehrformen, die Reihenfolge, in der die Studieninhalte präsentiert werden, die Integrationsleistungen von Forschung und Lehre sowie Theorie und Praxis.

1. Symbolische Bedeutungen: Die enge stereotypische Verbindung von Technik und Männlichkeit greift in der Sozialisation, insbesondere in westlichen Industrieländern ${ }^{6}$. Technische Kompetenz ist Teil der männlichen Geschlechtsidentität. Für Frauen hingegen gelten Stereotype als nicht technisch kompetent, dagegen mit sozialen und kommunikativen Kompetenzen ausgestattet. Im Studium mögliche Gegenmaßnahmen zum Unterlaufen dieser Stereotype sind alle inhaltlichen Verbindungen, die eine stärkere Sinnorientierung (auch von Almstrum 2002) als Hauptattraktor für Frauen in der Informatik identifiziert) in den Anwendungen der Informatik vermitteln, und indem von Anfang an die Studieninhalte entsprechend kontextualisiert werden. An der Carnegie Melleon University (CMU) in Pitts-

6 Als ,sehr interessiert“ an Technik bezeichneten sich selbst $42 \%$ der Jungen in Deutschland gegenüber nur $5 \%$ der Mädchen (Deutsche Shell 2000). 
burgh (Blum 2001) hat diese Berücksichtigung zu einer größeren Fülle von Studiengängen geführt, ohne dass dadurch die Personalanforderungen gestiegen wären.

2. Strukturelle Barrieren: Unsere „StudentInnenstudie“ (Schinzel 1999a) hat Studienstrukturen zutage gefördert, die Frauen beachteiligen: so etwa den Mangel an Programmierkursen zu Anfang des Studiums, der darin begründet ist, dass männliche Studierende entsprechende Erfahrungen mitbringen, was Frauen gleich zu Beginn, aber unnötigerweise, in eine defizitäre Position bringt; das Angebot an Lehrformen, das zu wenig offenere interaktive Veranstaltungen vorsieht, welche gerade von Frauen als effektiver wahrgenommen und bevorzugt werden; und nicht zuletzt der „computer talk“ der männlichen Studierenden, welcher vor allem zu Beginn des Studiums den Frauen den Eindruck vermittelt, nicht dazuzugehören, das „Eigentliche“, das aber mit dem Studium in Wahrheit nichts zu tun hat, nicht mitzubekommen, und sie damit an einer adäquaten Einschätzung von Erfolgserlebnissen behindert. An der CMU wurden ähnliche Beobachtungen gemacht und entsprechend viele und intensive Programmierkurse zu Beginn des Studiums eingerichtet. Die dortigen Maßnahmen, die wegen der großen Attraktivität der Informatik an der CMU nicht unbedingt alle auf die deutsche Situation übertragbar sind, haben zu einer Erhöhung des Frauenanteils von $7 \%$ auf nahezu die Hälfte der Studierenden geführt, wodurch sich das Studienklima von selbst ändert und der Hierarchien schaffende „computer talk“ ausbleibt.

3. Studienformen: Das Studium der Informatik ist an den meisten Universitäten in Vorlesungen, Übungen und nur wenigen Praktika organisiert. In der o.g. „StudentInnenstudie“ (Schinzel 1998 und 1999) zeigte sich, dass die informatischen Studienformen, vor allem Vorlesungen, insbesondere im Hauptstudium, eher männliche Bedürfnisse befriedigen, während die Kreativität und Kommunikation fördernden Seminare und Projekte offenbar Lerninteressen von Frauen eher entgegen kommen. Sicher können im Grundstudium klassische Lehrformen beibehalten werden, doch sollte in den Übungen und bei der Softwareherstellung in Praktika oder Studienarbeiten die Teambildung unterstützt werden. Spätestens im Hauptstudium aber sollten anstelle von engen verschulten Studienordnungen möglichst offene Formen treten, die die Integration der Studieninhalte ermöglichen. Die Diskussion und die Kommunikation sollten unterstützt werden und eine Vielfalt möglicher Veranstaltungsformen sollte offen gelassen werden. Proseminare, Seminare und Projektstudium ermöglichen es, grundsätzliche Fragen zu diskutieren und informatische 
Probleme aus verschiedenen Blickwinkeln zu beleuchten. In solchen Veranstaltungen ist es einfacher, Anwendungsprobleme auch von ihrer psychologischen, sozialen oder ökonomischen Dimension zu sehen, anstatt nur von der technischen Seite. Von daher können und sollten die zentralen informatischen Methoden entwickelt und diskutiert werden. Eine Veränderung der Studienorganisation in die vorgeschlagene Richtung käme nicht nur den Studentinnen, sondern allen Studierenden zugute, da die Lehrformen stark motivationsfördernd wirken und sich an den Erfordernissen der Softwareherstellung in der Arbeitswelt orientieren: Projektarbeit, Teamarbeit, Berücksichtigung des Anwendungskontextes und ökonomischer Dimensionen, Multiperspektivität etc. sind dort tägliches Brot.

Das Wichtigste aber ist das Klima: Studentinnen brechen weniger ihr Studium ab an Orten mit menschenfreundlicher kommunikativer Atmosphäre. Wissenschaftlerinnen kommen zumeist aus Schulen, in denen alle Menschen gut behandelt werden, Männer und Frauen. Und dies hilft dann auch der Qualität der Forschung, entgegen der weit verbreiteten Meinung, dass Konkurrenz den Druck erhöhe und damit die Güte der Forschung.

\section{Literatur}

Almstrum, Vicki L. (2002): What is the attraction to computing? In: Comm. ACM 46, 9, S. 51-55.

Augustine, Dolores L. (1999): The Socialist Silicon Ceiling: East German Women in Computer Science. In: Proceedings of the 1999 International Symposium on Technology and Society: Women and Technology: Historical, Societal, and Professional Perspectives, hg.v. April Brown/David Morton, S. 347-356.

Blum, Lenore (2001): Transforming the Culture of Computing at Carnegie Melon, http://www2.cs.cmu.edu/ lblum-/PAPERS/Transforming TheCulture.pdf, 21.3.2006.

Breckler, Steven. J./Wiggins, Elizabeth C. (1991): Cognitive Responses in Persuasion: Effective and Evaluative Determinants. In: Journal of Experimental Social Psychology 27, S. 180-200.

Burkhard, Anke: New Professors-Old Structures: Results of personnel replacement in East German universities from women's point of view. In: Women, Work and Computerization; Spinning a web from past to future; Proceedings of the 6 the Int. IFIP - Conference on Women, hg.v. Frances Grundy et al., Bonn, S. 31-44. 
Camp, Tracy (1997): The incredible shrinking pipeline. In: Communications of the ACM, Jg. 40, Nr. 10, S. 103-110.

Cisco (Academy for Educational Development, Cisco Learning Institute): Support for Gender Strategies in the Cisco Networking Academy Program in Ten Focus Countries, Gender Strategy, www. ciscolearning.org.

Crutzen, Cecile (2000): Interactie, een wereld van verschillen (Een visie op informatica vanuit genderstudies) Dissertation, Open universiteit, Heerlen.

Heintz, Bettina/Nadai, Eva (1998): Geschlecht und Kontext. DeInstitutionalisierungsprozesse und geschlechtliche Differenzierung. In: Zeitschrift für Soziologie, Jg. 27, Nr. 2, S. 75-93.

Huber, Birgit/Reiff, Isabelle/Ruiz Ben, Esther/Schinzel, Britta (2002): Frauen in IT- und ausgewählten technischen Ausbildungen und Berufen in Baden-Württemberg. Arbeitsbericht der Akademie für Technikfolgenabschätzung, Nr. 213, Stuttgart.

Lovegrove, Gillian/Segal, Barbara (Hg.) (1991): Women into Computing, Selected Papers 1988-1990. Workshops in Computing, Berlin/Heidelberg/New York.

Roloff, Christina (1989): Von der Schmiegsamkeit zur Einmischung. Professionalisierung der Chemikerinnen und Informatikerinnen, Pfaffenweiler.

Schinzel, Britta (1997): Why is female participation decreasing in German Informatics? In: IFIP-Conference on Women Work and Computerization, Berlin/Heidelberg/New York.

Schinzel, Britta/Klein, Karin, Wegerle, Andrea/Zimmer, Christine (1999a): Das Studium der Informatik-Studiensituation von Studentinnen und Studenten. In: Informatik-Spektrum, Nr. 22, S.13-23.

Schinzel, Britta (1999b): The Contingent construction of the relation between gender and computer science. In: Proceedings of the 1999 International Symposium on Technology and Society: Women and Technology: Historical, Societal, and Professional Perspectives, hg.v. April Brown/David Morton, S. 299-312.

Schinzel, Britta (2000): Cross country Computer Science Students' Study: an analysis of differences in the situation of male and female students in Computer Science between several countries. In: $C D$ Proceedings of the WWC-Conference, S.30 ff.

Schinzel, Britta (2001): Be-Deutungen der Informatik als Ingenieurswissenschaft In: Zeitschrift für Kommunikationsökologie, Nr. 3, S. 27-41.

Schinzel, Britta (2002a): Female enrolment in tertiary education in Computer Science. In: Improving the gender balance in engineering 
education using ICT methods and contents, hg.v. Katarina Alha, Brüssel S. 103-114.

Schinzel, Britta. (2002b): Cultural differences of female enrolment in tertiary education in Computer Science. In: Human Choice and Computers, Proceedings of the 17th World Computer Congress, hg.v. Klaus Brunnstein/Jacques Berleur, Montreal, S. 283-292.

Schinzel, Britta (2003a): Curriculare Vorschläge zur Erhöhung des Frauenanteils in der Informatik. Möglichkeiten und Maßnahmen, http://mod.iig.uni-freiburg.de/publikationen/publ2003.html.

Schinzel, Britta (2003b): Geschlechterverhältnis und Technikentwicklung. In: Dokumentation wissenschaftliche Kolloquien 1999-2002, Wissenschaftliche Reihe NFFG; Bd. 2, hg.v. Ursula Paravicini/Maren Zempel-Gino, Hannover, S. 91-97.

Schinzel, Britta/Ruiz Ben, Ester (2004): Softwareentwicklung als Beruf? Professionalisierungstendenzen und Implikationen für die Beteiligung von Frauen. In: Informatik-Spektrum Bd. 27, Nr. 5, S. 441447.

Zachmann, Karin (1997): Frauen für die Technische Revolution. Studentinnen und Absolventinnen Technischer Hochschulen in der SBZ/DDR. In: Frauen arbeiten. Weibliche Erwerbstätigkeit in Ostund Westdeutschland nach 1945, hg.v. Gunilla Budde, Göttingen. 



\title{
Geschlechtergerechtes Lehren und
}

\section{Lernen in Naturwissenschaft und Technik.}

\author{
Aktiv, kooperativ und authentisch \\ durch Kontextorientierung und \\ reflexive Koedukation
}

MONIKA BESSENRODT-WEBERPALS

Beim Lehren und Lernen in den Naturwissenschaften unterscheiden sich Studentinnen und Studenten deutlich sowohl in ihrem Arbeitsverhalten als auch in ihren inhaltlichen Interessen. Diese genderdifferente Sichtweise gilt es für die Fachdidaktik nutzbar zu machen, um den StudienErfolg nachhaltig zu verbessern.

\section{Prolog}

Zur Einstimmung sei zunächst kurz die besondere Situation im Bereich Gender Studies in Hamburg geschildert. Hier arbeiten hochschulübergreifend sieben Hamburger Hochschulen zusammen, nämlich die Universität, die Hochschule für Angewandte Wissenschaften (HAW), die Technische Universität, die Hochschule für bildende Künste, die Evangelische Fachhochschule, die Helmut-Schmidt-Universität der Bundeswehr und die Hochschule für Musik und Theater. Dank des HochschulWissenschafts-Programmes des BMBF konnte in Hamburg einmalig für Deutschland der Schwerpunkt „Gender and Science“ etabliert werden. Dafür vertreten an drei Hochschulen insgesamt vier Professorinnen jeweils Gender Studies in Kombination mit einem Fach aus Mathematik- 
Informatik-Naturwissenschaften-Technik (MINT-Fach), konkret mit Mathematik, Informatik, Physik und Ingenieurwissenschaften. ${ }^{1}$

Mein eigenes Arbeitsfeld in „Gender and Science“ ist das Lehren und Lernen in Naturwissenschaft und Technik, insbesondere der Physik. Diesem Themenkreis entstammt auch der folgende Beitrag.

\section{Einführung und Überblick}

Dieser Beitrag stellt dar, wie Kontextorientierung geschlechtergerechte Lehre in den MINT-Fächern und insbesondere in der Physik ermöglicht. Dafür werden die Rahmenbedingungen und Strukturen für die Hochschullehre von Studentinnen und Studenten im Sinne von Gender Mainstreaming unter Berücksichtigung der Unterschiede, also von Diversity, untersucht. Konsequent setzen die Strategien inhaltlich wie organisatorisch auf Vielfalt und Chancengerechtigkeit, kurzum auf eine optimale Lehre für alle in der Hochschule.

Zuerst sei dieses Thema in die aktuellen Diskussionen zum Thema Studienmisserfolg eingebettet. Die große Studienabbruchstudie vom Hochschulinformationsdienst aus Hannover aus dem Jahr 2005 (Heublein/ Schmelzer/Sommer 2005) hat die Studienabbruchquote berechnet, also den prozentualen Anteil derjenigen, die nie irgendeinen Hochschulabschluss erreichen. Für die Ingenieurwissenschaften wie die MINTFächer liegen die Studienabbruchquoten der deutschen Studierenden an Universitäten bei etwa $30 \%$, wobei mehr Studenten ihr Studium abbrechen als Studentinnen. Für die einzelnen Fächer sind die Abbruchquoten recht unterschiedlich, aber leider nicht geschlechtersensitiv aufgeschlüsselt.

In einer ergänzenden Studie hat Dagmar Richter (Richter 2006) an der HAW Hamburg den Zeitpunkt des Studienabbruches präzisieren können und zusätzlich Studienabbrecherinnen und -abbrecher nach den Gründen für ihren Misserfolg befragt. In beiden untersuchten Fachbereichen Naturwissenschaftliche Technik und Elektronik und Informatik zeigten sich Geschlechterunterschiede: Studentinnen brechen früher ihr Studium ab als Studenten. Als prioritäre Studienabbruch-Gründe werden von Studienabbrecherinnen angegeben, dass sie das falsche Studienfach gewählt hätten bzw. ihre Kompetenz nicht anerkannt worden sei. Studienabbre-

1 Weitere Informationen sind unter der URL http://www.genderstudieshamburg.de zu finden. 
cher dagegen berichten über fehlende Lernpraxis oder prioritäre Erwerbsarbeit. Beide Geschlechter gemeinsam monieren die unbefriedigende Lehr-Lern-Situation, was die zentrale Bedeutung dieses Beitrages für den Studienerfolg unterstreicht.

In Hinblick auf mögliche Empfehlungen für Studienerfolg wird als nächstes im folgenden zweiten Kapitel die genderdifferente Sicht auf die MINT-Fächer vergegenwärtigt, denn „Wissenschaft wird von Menschen gemacht", wie schon Werner Heisenberg festgestellt hat. Dem wird im dritten Kapitel kontextorientiertes Lehren und Lernen an die Seite gestellt. Im vierten Kapitel wird diese Kontextorientierung für meine physikalischen Lehrveranstaltungen im Detail beschrieben, und es werden praktische Hinweise zur Umsetzung gegeben.

Schließlich werden die Beobachtungen zu Empfehlungen im fünften Kapitel zusammengefasst.

\section{Genderdifferente Sichtweisen auf MINT-Fächer}

Zahlreiche Untersuchungen an Jugendlichen in der Schule belegen, dass es markante geschlechtsdifferente Unterschiede im Zugang zur und im Umgang mit Naturwissenschaften und Technik gibt. Diese betreffen unterrichtsrelevante Fähigkeiten wie das räumliche Vorstellungsvermögen, eine unterschiedliche Motivation für Fachinhalte (Mädchen bevorzugen Biologie, Jungen Technik) sowie das mehr oder weniger dominante oder aggressive Verhalten im Unterricht (vgl. Martial 2003).

Fragen nach dem Image von MINT-Schulfächern zeigen (Kessels et al. 2002), dass Physik und Mathematik als schwierig und wenig sinnlich gelten. Leute mit Lieblingsfach Mathematik oder Physik werden zwar als intelligenter und leistungsmotivierter eingeschätzt als Leute mit Lieblingsfach Deutsch oder Sprachen, aber auch als weniger attraktiv, weniger sozial kompetent und weniger kreativ. Während das Fach Deutsch von $81 \%$ der Befragten als neutral angesehen wird, gilt das immerhin noch für $71 \%$ der Befragten auch für das Fach Mathematik aber nur für $46 \%$ für das Fach Physik. Mehr, nämlich $47 \%$, meinen, Physik sei ein Jungenfach. Übrigens stellt nur für eine kleine Minderheit von wenigen Prozent Physik und Mathematik ein Mädchenfach dar (vgl. ebd.).

Die genderdifferente Sichtweise auf die Physik stellt die Untersuchung der Gruppe von Helga Stadler (Stadler et al. 2000) an 16-jährigen Gym- 
nasiastinnen und Gymnasiasten vor. Mädchen fragen eher: „Warum?“, Jungen eher: „Wie?“. Mädchen antworten lieber auf offene Fragen, indem sie in ihrer Alltagssprache (häufig anthropomorph) komplette Sätze formulieren, während Jungen enge Ja/Nein-Fragen bevorzugt beantworten und im Telegrammstil Fachvokabeln anbieten. Auch in der Gruppenarbeit gibt es signifikante Unterschiede, da Mädchen eher Fragen stellen und auch ihre Unkenntnisse zeigen, hingegen Jungen Stärke demonstrieren wollen und befehlen. Mädchen suchen zudem häufiger nach einem weiteren Anwendungsfeld einer Aufgabe, Jungen zielen stärker auf die konkrete Lösung. Damit wirkt die Physik bei Mädchen überwiegend durch ihre Beziehung zur Welt, d. h. durch externe Kohärenz, und bei Jungen eher als Selbstzweck, d. h. durch interne Kohärenz.

Externe Kohärenz, also die Vernetzung von Wissen durch das Anknüpfen an Vorwissen, steht an zentraler Stelle und mehrfach in den zehn Punkten für guten Physikunterricht, die Häussler und Hoffmann in einem IPN-Modellversuch (Häussler/Hoffmann 1995) an acht Gymnasien in Schleswig-Holstein extrahiert haben:

1. Neugier wecken! Aha-Erlebnisse

2. An außerschulische Erfahrungen anknüpfen!

3. Aktives und eigenständiges Handeln fördern!

4. Bezüge zur Alltagswelt herstellen!

5. Bedeutung der Naturwissenschaften für die Gesellschaft verdeutlichen!

6. Lebenspraktischen Nutzen der Naturwissenschaften erfahrbar machen!

7. Bezug zum eigenen Körper herstellen! Mit allen Sinnen lernen!

8. Begriffliches Verständnis vor dem Aufstellen von Gleichungen fördern!

9. Notwendigkeit und Nutzen quantitativer Größen verdeutlichen!

10. Spielerisches Umgehen und unmittelbares Erleben stärken!

Dieses Unterrichtskonzept bewährt sich in der Schulpraxis, wo es einen signifikant höheren Wissensstand der Schülerinnen und Schüler erreicht bei einem gleichzeitig deutlich geringeren Interessenschwund. Und hiervon profitieren besonders die Mädchen, deren Motivation sogar zunimmt, die eine positivere Einstellung gegenüber der Physik im Alltag und zugleich ein positiveres Selbstkonzept entwickeln. Im Ergebnis lassen sich höhere kognitive und affektive Lernerfolge von Mädchen und Jungen erzielen. Auch an außerschulischen Lernorten wie Schülerlabors zeigt dieses aktive Lernen keinen Gender-Gap (Engeln/Euler 2004). Damit lässt sich Geschlechtergerechtigkeit erreichen und gleichzeitig 
nach Martin Wagenschein neu interpretieren, der geschrieben hat: „Ich habe im Koedukationsunterricht immer die Erfahrungen gemacht, wenn man sich nach den Mädchen richtet, so ist es auch für die Jungen richtig, umgekehrt aber nicht" (Wagenschein 1965).

Wie steht es nun mit der geschlechtergerechten Hochschullehre in den MINT-Fächern? Dafür habe ich Studierende der Medientechnik an der HAW Hamburg zum Lehren und Lernen von Physik befragt. Weit mehr als die Hälfte der Studierenden meiner Umfrage, Frauen und Männer, schätzen Praxisbezüge und aktivierenden Unterricht hoch bis sehr hoch ein. Studentinnen (63 bis $88 \%$ ) legen noch größeren Wert als Studenten (55 bis $79 \%$ ) auf externe Kohärenz - zum Beispiel Lernen durch Analogien und Einbettung in Alltagsphysik. Jacquelynne Eccles (Eccles 2003) stellt Analoges in ihrer Studie an der University of Michigan, Ann Arbor, fest. Auch die höheren Frauenanteile in den neuen BindestrichStudiengängen in der Physik (zum Beispiel in Medizin-Physik, BioPhysik) deuten in diese Richtung.

\section{Kontextorientiertes Lehren und Lernen}

An den Massenuniversitäten ist die Lehre geprägt von deklarativer Lehre im Sinne einer Vorlesung. Statt sich auf diese enge Vermittlung von Fakten und Inhalten zu beschränken, setzt kontextorientierte Lehre auf deren Einbettung in eine authentische Lehr-Lern-Umgebung und betrachtet vor allem Kontexte und Prozesse, die zum Wissenserwerb führen, womöglich in Projektarbeit. Denn Lernen ist ein individueller Vorgang, den wir nicht durch Instruktion vermöge eines Nürnberger Trichters beschleunigen können, vielmehr findet eine subjektive Konstruktion von Wissen im je eigenen Kopf statt. In der Tat gehört zu den unbestrittenen Maximen der Lernpsychologie, dass jegliches Lernen vom Lernenden selbst auszugehen hat. Hierunter fällt auch das autonome Lernen mit seinem von Leo van Lier formulierten Triple-A von „Autonomy Authenticity - Awareness“ (Autonomie - Authentizität - Aufmerksamkeit) (Lier 1996).

Damit lassen sich auch die erforderlichen Kompetenzen in der Wissensgesellschaft erwerben:

- deklaratives (Sach-/Fakten-)Wissen

- prozedurales (Verfahrens-)Wissen

- metakognitives (Kontroll-/Steuerungs-)Wissen über Lern- und Denkprozesse

- wertebezogenes (Orientierungs-)Wissen 
Nach Martin Wagenschein bietet sich zur Vertiefung forschendentdeckendes Lernen an ausgewählten Beispielen an (Wagenschein 1965). Die ganzheitliche, handlungsorientierte Lehre setzt auf das Lernen mit Hirn, Herz und Hand, ist also gleichzeitig kognitiv, emotional und praktisch orientiert (Möller/Tenberge 1997). In der Umsetzung in der Hochschullehre bevorzuge ich als Quintessenz dieser Überlegungen das multiperspektivische Lernen mit allen Sinnen, wie es auf anderer Ebene auch die Regionale Ökologische Sachunterrichtslernwerkstatt RÖSA von Astrid Kaiser an der Universität Oldenburg praktiziert ${ }^{2}$ (Kaiser 2002). Hier geht spielerisches Üben einher mit einer Förderung der Kreativität der Lernenden. Sie erhalten Informationen von den Lehrenden und müssen sich selbst weitere Informationen beschaffen, über die sie ihrerseits wieder kommunizieren. Damit wird sowohl ihre Selbstständigkeit unterstützt als auch ihre Fähigkeit geschult, kooperativ im Team zu arbeiten. Experimentierend machen die Lernenden neue Entdeckungen, die sie forschend entsprechend ihren Interessen und Fähigkeiten vertiefen können. Ihre Ästhetik wird ebenso einbezogen wie die Erkenntnis, dass sich durch Handeln Missstände verändern lassen. Eine Spezialform von kontextorientiertem Lernen ist Problemorientiertes Lehren und Lernen, das zunehmend im Medizinstudium eingesetzt wird.

\section{Eigenes Konzept und seine Umsetzung}

\section{„Aktiv sein ist (fast) alles!“ (Bessenrodt-Weberpals 2007)}

Mein Konzept des Lehrens und Lernens an der Hochschule basiert auf kontext- und handlungsorientiertem Lernen mit reflexiver Koedukation im Sinne von Gender Mainstreaming und Diversity. Das Konzept setzt auf ganzheitliches Lernen mit Hand, Herz und Hirn, ist also praktisch, emotional und kognitiv auf das Lernen mit allen Sinnen ausgerichtet. Es wählt den lernzentrierten Zugang in authentischer Umgebung, indem es fächerübergreifendes Lernen mit minimaler Formalisierung verfolgt. Indem ich mit Alltagsmaterialien experimentieren lasse, wird zugleich an die authentischen Alltagserfahrungen der Lernenden angeknüpft und ein niedriger Preis ermöglicht. Zudem ist dieser Zugang ressourcenschonend und stärkt den Umweltgedanken. Mein Ziel ist, Motivation durch Erfolg zu erreichen. Studienbegleitende Prüfungen mit einer zeitnahen Rückkopplung des Kenntnisstandes gehören dazu, wie es auch die Akkreditierung beim Bachelor verlangt.

2 Siehe auch www.roesa.de 
Als Folge dieses Konzeptes wird die Verbindung zwischen Gender und Science fester geknüpft. Es gelingt eine adaptive Konstruktion von Wissen, die sich am Kontext der naturwissenschaftlichen Phänomene orientiert und gleichzeitig autonomes Lernen fördert, das heißt: Jede Person kann sich entsprechend ihres Vorwissens, ihrer Interessen, Begabungen und Fähigkeiten in die Studieninhalte vertiefen. Die Zielorientierung dieses Konzeptes spiegelt sich sowohl im fertigen Produkt wieder als auch in der weiten Anwendung von Wissen und sorgt damit für vernetztes Denken und die nötigen Kompetenzen in der Wissensgesellschaft. Und damit geht solches kontextorientiertes Lernen auf die Vielfalt der Lernenden und ihre unterschiedlichen Begabungen ein, Frauen wie Männer - mit oder ohne Migrationshintergrund - können sich dadurch entfalten. So ist eine Reform von MINT- Studiengängen zugunsten von Frauen auch ohne monoedukative Studiengänge möglich (vgl. FaulstichWieland 2006).

Der praktische Umgang mit den Handlungsmaterialien stützt sich auf die Ideen der offenen Unterrichtsformen (Hagstedt 1992). Dazu zählen Lernwerkstatt, Lernbuffet und Stationenlernen, das wie ein sportliches Zirkeltraining aufgebaut ist, ebenso wie Ergänzungselemente zu konventionellem Unterricht. Die lernzentrierte Vielfalt von Methoden benutzt unter anderem Open-Air-Unterricht, biografische Ausgangspunkte, Präsentationstechniken und Multimedia ebenso wie Lernen im Team (vgl. Frank 1997).

Die Umsetzung dieses Konzeptes in den Naturwissenschaften stellt sich als sehr erfolgreich heraus, insbesondere bei den Studentinnen. Die Bandbreite ist groß: An der HAW Hamburg profitieren zurzeit die Studierenden des Fachbereichs Medientechnik von dieser innovativen Art, Physik zu lernen. Damit nähern sich interdisziplinäre didaktische Ziele ihrer Verwirklichung, wie sie auch im Internationalen Frauenstudiengang Informatik geplant sind (Müller/Wetzel 2006).

Darüber hinaus sollten diese Argumente verstärkt Eingang in die Hochschulausbildung der künftigen Lehrkräfte in den MINT-Fächern finden. Paradigmatisch sei hier auf die entsprechende Studie der Deutschen Physikalischen Gesellschaft verwiesen (Großmann/Urban 2006). 


\section{Zusammenfassung und Ausblick}

Kommen wir nun zurück auf unser Ziel, Empfehlungen zum Studienerfolg in den MINT-Fächern zu formulieren, und betrachten die verschiedenen Akteurinnen und Akteure:

Studierende sollten informiert, motiviert und fähig für ihr gewähltes Studienfach sein und die notwendigen Kenntnisse dafür in der Schule erworben haben. Insbesondere die jungen Frauen sollten ausreichend selbstbewusst auch in schwierigen Situationen sein, damit sie nicht durch eine mangelnde Akzeptanz ihrer Kompetenzen entmutigt werden.

Lehrende an der Hochschule unterrichten inhaltlich und didaktisch kompetent. Sie knüpfen an Vorerfahrungen der Studierenden an, insbesondere in interdisziplinären Projekten, und arbeiten mit vielen aktivierenden Elementen, die alle Sinne ansprechen. Sie stärken das Selbstbewusstsein der Studierenden, besonders der jungen Frauen. Den Lehrenden kommt eine herausgehobene Rolle als wichtige Lernhelferinnen und Lernhelfer $\mathrm{zu}$.

Zusätzlich kommen die Leitungsgremien an der Hochschule ins Spiel, die eine stärkere Zusammenarbeit mit Schulen wie mit der Wirtschaft fördern. Ersteres kann durch umfassende Informationen zu Studienfächern, eine faire Studierendenauswahl nach Passung und Zusatzangebote wie Mädchentechniktage bereits in der Mittelstufe, jedenfalls vor der Wahl der Leistungskurse, erfolgen. Hinzu treten inhaltliche und organisatorische Studienreformmaßnahmen.

Interdisziplinäre, kontextorientierte Studieninhalte, die auf die Gesellschaft bezogen sind, und transdisziplinäre Schlüsselqualifikationen (soft skills) zählen ebenso dazu wie Projekt- und Teamarbeit, studienbegleitende Prüfungen und Anreizsysteme für Gender Mainstreaming und Diversity. Diese umfassen reflexive Koedukation in Lehrveranstaltungen, d. h. geschulte Lehrende in Gender-Themen, und (phasenweise) Monoedukation in Einzelfällen, sowie auch mehr weibliches Lehrpersonal, das als Vorbild besonders für die Studentinnen wirken kann. Zusätzlich lassen sich interdisziplinäre Inhalte und Strukturen der Geschlechterforschung bezogen auf physikalische Felder integrieren (BessenrodtWeberpals 2006).

Insgesamt bereitet dieses Vorgehen auf die gefragten Schlüsselkompetenzen in der Wissensgesellschaft vor und trifft die Erwartungen vom 
Deutschem Industrie- und Handelstag (DIHK 2004) und der 19-PunkteErklärung des VDI (VDI 2002).

Gerade jetzt bietet sich die Chance, die Umstellung auf Bachelor- und Masterstudien als Prototyp für eine erfolgreiche Studienreform zu nutzen!

\section{Literatur}

Bessenrodt-Weberpals, Monika (2006): Gender und Physik. In: Gender - Aspekte bei der Einführung und Akkreditierung gestufter Studiengänge - eine Handreichung, hg.v. Ruth Becker et al., Netzwerk Frauenforschung NRW, Heft 7, Dortmund, S. 99-102.

Bessenrodt-Weberpals, Monika (2007): Experimentieren und Entdecken: Experimente für den Physikunterricht (Praxisbuch in Vorbereitung).

Deutscher Industrie- und Handelstag (2004): Fachliches Können und Persönlichkeit sind gefragt.

Eccles, Jacquelynne (2003): U-M study helps define why fewer women choose math-based careers, University of Michigan, Ann Arbor.

Engeln, Katrin/Euler, Manfred (2004): Forschen statt Pauken. In: Phys. J., 3. Jg., Heft 11, S. 45-48.

Faulstich-Wieland, Hannelore (2006): Monoedukative Studiengänge Ultima Ratio für die Veränderung der Geschlechterverhältnisse in technischen Studiengängen. In: Hochschulinnovation. Genderinitiativen in der Technik, hg.v. Carmen Gransee, Hamburg (im Druck).

Frank, Elisabeth (1997): Schule der Chancengleichheit. Impulse für eine qualifizierte Koedukation am Beispiel des Schulversuchs Physik, Landesinstitut für Erziehung und Unterricht, Stuttgart.

Großmann, Siegfried/Urban, Knut (2006): Thesen für ein modernes Lehramtsstudium im Fach Physik, Deutsche Physikalische Gesellschaft, Bad Honnef.

Hagstedt, Herbert (1992): Offene Unterrichtsformen. Methodische Modelle und ihre Planbarkeit. In: Innovationsprozesse in der Grundschule, hg.v. Uwe Hameyer et al., Bad Heilbrunn, S. 367-382.

Häussler, Peter/Hoffmann, Lore (1995): Physikunterricht an den Interessen von Mädchen und Jungen orientiert. In: Unterrichtswissenschaft 23, S. 107-126.

Heublein, Ulrich/Schmelzer, Robert/Sommer, Dieter (2005): Studienabbruchstudie 2005, Hochschul-Informations-System, Heft A1, Hannover. 
Kaiser, Astrid (2002): Einführung in die Didaktik des Sachunterrichts. 8. Aufl., Baltmannsweiler.

Kessels, Ursula/Hannover, Bettina/Rau, Melanie/Schirner, Sigrun (2002): Ist die Physik reif für eine Image-Kampagne? In: Phys. J., Jg. 1, Heft 1, S. 65-68.

Lier, Leo van (1996): Interaction in the Language Curriculum: Awareness, Autonomy, and Authenticity, London.

Martial, Ingbert von (2003): Koedukation und getrennte Erziehung. Pädagogik und freie Schule, Heft 51.

Möller, Kornelia/Tenberge, Claudia (1997): Handlungsintensives Lernen und Aufbau von Selbstvertrauen im Sachunterricht. In: Forschung zum Sachunterricht. Probleme und Perspektiven des Sachunterrichtes, hg.v. Brunhilde Marquart-Mau/Walter Köhnlein,/Roland Lauterbach, Bad Heilbrunn, S. 134-153.

Müller, Anna/Wetzel, Ingrid (2006): Eine „Auszeit“ von Geschlechterdifferenzen. Der Internationale Frauenstudiengang Informatik setzt auf genderadäquate Didaktik. In: Hochschulinnovation. Genderinitiativen in der Technik, hg.v. Carmen Gransee, Hamburg (im Druck).

Richter, Dagmar (2006): „...und dann ist das gar keine Schule!“ Studienabbruch-Gründe von Frauen und Männern in Ingenieurwissenschaften. Ein Projektbericht aus der Hochschule für Angewandte Wissenschaften Hamburg. In: Hochschulinnovation. Genderinitiativen in der Technik, hg.v. Carmen Gransee, Hamburg (im Druck).

Stadler, Helga/Duilt, Reinders/Benke, Gertrud (2000): Do boys and girls understand physics differently? In: Phys. Educ., Jg. 35, Heft 6, S. 417-422.

VDI - Verein deutscher Ingenieure (2002): Ingenieure und Ingenieurinnen in Deutschland. Situation und Perspektiven, Düsseldorf, S. 7.

Wagenschein, Martin (1965): Ursprüngliches Verstehen und Exaktes Denken, Stuttgart. 


\section{Gender und Diversity in}

\section{Ingenieurwissenschaften}

SUSANNE IHSEN

„Diversity“ (Vielfalt) heißt das neue Zauberwort. Entstanden ist die betriebliche Berücksichtigung verschiedener Personengruppen allerdings nicht bei der Klärung der Geschlechterfrage. Vielmehr sahen weltweit operierende Unternehmen sich vor dem Managementproblem, globale Strategien und lokale Märkte zusammen zu bringen. Die Vielfalt wird nun bei den Ansprüchen von Kundinnen und Kunden sowie bei Märkten in unterschiedlichen Kulturen und Regionen nutzbar gemacht. Gemeinsam mit der Erkenntnis in Unternehmen, dass künftig nicht nur Fachfrauen fehlen, sondern dass insgesamt zu wenige Ingenieurinnen und Ingenieure zur Verfügung stehen, führt das dazu, eine Vielzahl von Programmen und Maßnahmen aufzulegen, um bis in einzelne Entwicklungsteams hinein diese unterschiedlichen Kundengruppen $\mathrm{zu}$,spiegeln“. In einem zweiten Schritt führt dieser neue Forschungs- und Entwicklungsansatz $\mathrm{zu}$ personalpolitischen Konsequenzen, zu maßgeschneiderten Arbeitszeitmodellen, zu Programmen rund um „Work-LifeBalance“.

\section{Einleitung}

Zurzeit können wir zwei Argumentationslinien identifizieren, mit denen eine stärkere Berücksichtigung bislang nicht erreichter Menschen für Ingenieurberufe begründet werden: Der zu erwartende demografische Wandel und ein prognostizierter Fachkräftemangel in technischen Berufen rücken die ökonomischen und politischen Dimensionen in den Blick. Und der „Diversity“-Ansatz geht davon aus, dass sich Prozesse von der Entwicklung bis zur Vermarktung verändern, wenn sich statt der bisherigen relativ homogenen Männergruppen gemischte Teams mit den Wünschen von Kundinnen und Kunden befassen, sucht zunächst die 
technisch ausgebildete Fachfrau, dann auch Vertreterinnen und Vertreter verschiedener Generationen und aus unterschiedlichen Herkunftskulturen.

Die Ansätze, mehr Frauen in technische Berufe zu bekommen, sind damit in der Gesellschaft und in den Unternehmen angekommen. Es geht nicht ausschließlich um Gleichberechtigung und Chancengleichheit, es geht um klar bezifferbare volks- und betriebswirtschaftliche Größen: Dies beinhaltet, neben der (zu) geringen Beteiligung von Frauen in den Ingenieurwissenschaften, die über Jahrzehnte fast unverändert gebliebene ingenieurwissenschaftliche Fachkultur (vgl. u.a. Molvaer/ Stein 1994: 93ff) sowie die mehrheitlich noch immer fehlenden, aber unternehmerisch geforderten Kompetenzprofile von Hochschulabsolventen und Hochschulabsolventinnen der Ingenieurwissenschaften (vgl. Brödner 1996; VDI 1995; 2004). Arbeitsgruppen, die nicht mehr homogen, sondern, die industrielle und gesellschaftliche Realität widerspiegelnd, „mixed“ sein sollen, scheitern aber oft genug daran, dass sich noch immer nur eine sehr homogene Gruppe der Studierenden zu einem solchen Studium motivieren lässt: Studierende sind mehrheitlich Männer, an traditionellen Berufsfeldern interessiert, und sie kommen häufig aus einem bereits technisch vorgeprägten Umfeld (vgl. Molvaer/Stein 1994).

\section{Das Fachgebiet Gender Studies in Ingenieurwissenschaften der TU München}

Die Technische Universität München (TUM) befindet sich bereits seit einiger Zeit in einem Reorganisationsprozess, ähnlich dem von Unternehmen, weg von einer nachgeordneten Behörde hin zu einem modernen, flexiblen „Wissenschaftsbetrieb“. Der TUM ist daran gelegen, die aktuellen Veränderungen im wissenschaftspolitischen Raum (Bachelor/ Master, Föderalismus usw.) zu nutzen, um ihr international anerkanntes Profil in Forschung und Lehre durch eine unternehmensähnliche Organisationsstruktur weiter zu schärfen. Zu diesem Gesamtkonzept gehört seit 2004 ein Konzept der ,gendergerechten Hochschule“, getragen durch Hochschulleitung, Frauenbeauftragte und Verwaltungsrat. Ziel war und ist, alle Einzelmaßnahmen, Programme und Aktivitäten in ein gemeinsames Zielsystem zu integrieren und so die Umsetzung des Gender Mainstreaming an der TUM voranzutreiben (vgl. TUM 2004). Ziel des Gleichstellungskonzeptes ist, zunächst die vom Gesetzgeber vorgeschriebene, gleichberechtigte Teilhabe von Frauen und Männern im wissenschaftlichen Bereich an der TUM. Darüber hinaus möchte die einzige 
voll ausgebaute technische Hochschule in Bayern, mit den daraus resultierenden niedrigen Frauenanteilen in Studium und Wissenschaft, nicht nur eine nachhaltige Stabilisierung und Steigerung dieser Frauenanteile erreichen, sondern auch qualitativ durch die Berücksichtigung von Gender-Aspekten die Gestaltung von Naturwissenschaft und Technik verändern.

„In einer TUM-spezifischen Ausprägung von Gender Mainstreaming verfolgen wir die Kernstrategie, einen Transfer von gender-politischem Fachwissen, von erfolgreichen Strategien zur Gleichstellung und von attraktiven Ideen und erprobten Konzeptionen für Gender-Projekte in alle Bereiche der Hochschule sicherzustellen. Gender-Gerechtigkeit soll durchgängig und nachhaltig als Querschnittsthema an der TUM etabliert und in unserer Corporate Identity verankert werden“ (Prof. Dr. Dr. h.c.mult. Wolfgang A. Herrmann, Präsident der TUM; TUM 2004).

Dieses Gesamtkonzept setzt auf den genderspezifischen Projekten der Frauenbeauftragten auf (Mentoring- und Schülerinnenprogramme, Ferienakademie, und Career Center). Davon ausgehend wurden Konzepte für eine spezifische Förderung des wissenschaftlichen Nachwuchses, im Fundraising, im Hochschulentwicklungsplan, in der Kinderbetreuung und in Konzepten zur Vereinbarkeit von Beruf und Familie entwickelt und umgesetzt.

Im Dezember 2004 wurde schließlich die bislang bundesweit einzige Professur für Gender Studies in Ingenieurwissenschaften besetzt und interdisziplinär in der Fakultät Elektrotechnik und Informationstechnik eingerichtet. Das Fachgebiet „Gender Studies in Ingenieurwissenschaften“ trägt dazu bei, die Ursachen des geringen Frauenanteils in den Ingenieurstudiengängen zu erkennen, Maßnahmen zur Veränderung zu entwickeln und zu überprüfen. Dazu gehören Analyse und Veränderungspotenziale bei den Berufs- und Karriereverläufen von Ingenieurinnen ebenso wie eine Untersuchung der Frage, wie sich das Interesse an Technik und ihrer Entwicklung bei Frauen und Männern ausprägt. Zurzeit entdecken Unternehmen, dass die Wahrnehmung von vielfältigen Kundeninteressen zu einer Produktoptimierung und -weiterentwicklung führt. Um diese Vielfalt („Diversity“) zu erzielen, setzen sie auf heterogene Entwicklungsteams: Frauen und Männer, Menschen aus unterschiedlichen Generationen und mit verschiedenen Herkunftskulturen.

Auch für die Arbeits- und Unternehmenskultur gilt, einseitig geprägte Strukturen zu identifizieren und - wenn sie ungleiche Chancen für Frauen oder Männer zur Folge haben - an Veränderungen mitzuwirken. 
Für Frauen - aber auch für Männer - sollen so neue Wege eröffnet werden, ihr Berufsleben erfolgreich und zufrieden zu gestalten. Die Aufgaben und Angebote des Fachgebiets umfassen sowohl Forschung, Lehre, als auch Angebote für Unternehmen. Die Arbeitsschwerpunkte sind:

- Gender und Diversity im Studium

- Gender und Diversity im Beruf

- Gender und Diversity an der Hochschule

- Gender und Diversity in der Technikentwicklung

- Diversity-Management in Unternehmen.

\section{Entstehung, Erhalt und Veränderung einer Technikkultur}

\section{Kulturen und ihre Grenzen}

Erfolg und Misserfolg von Reformprozessen sind eng mit der Veränderung der Fachkultur verbunden. Jede Fachkultur verfügt z.B. über eine eigene Geschichte, Zukunftsvisionen, Strukturen und Menschen, aber auch über Tabus, Widersprüche und Konflikte (Ihsen 1999, Abb. 1).

Abbildung 1: Ein soziales System

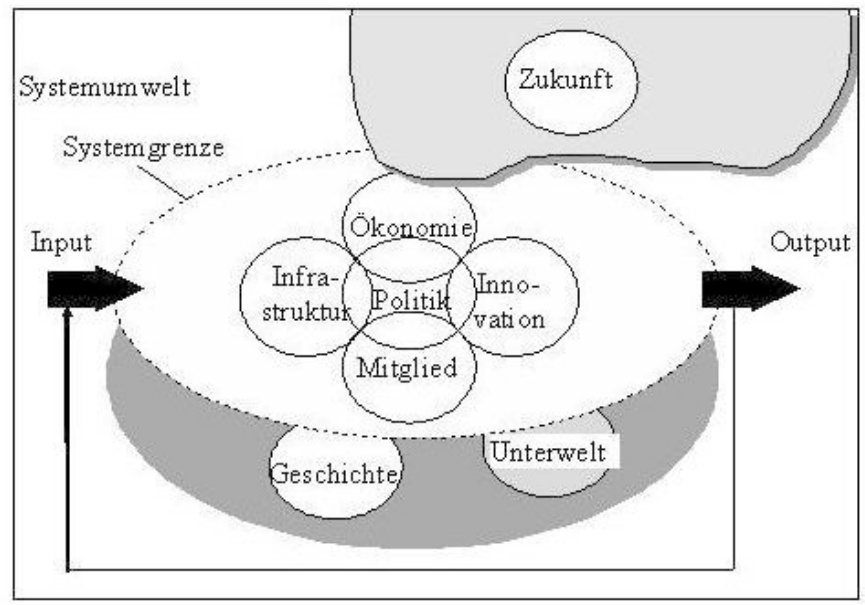

Quelle: Rieckmann/Weissengruber 1990: 42 
Eine solche Kultur produziert und vermittelt einen eigenen Sinn, indem verschiedene Leitideen ausgewählt werden (vgl. Rehberg 1992: 12) und damit innerhalb dieser Kultur gesetzesähnlich werden. Die Leitideen sind in Zielen, Strategien und Grundsätzen zu finden, wie auch in der Form der Machtverteilung, Führungsgrundsätzen, Funktionsrollen und „typischen Einstellungen“.

Um an die Mitglieder des Faches vermittelt zu werden, braucht die Kultur einen institutionellen Rahmen, z. B. spezielle Fachbereiche an Hochschulen, primär technisch geprägte Unternehmensbereiche und $\mathrm{Be}$ rufsverbände.

\section{Wahrnehmungsdefizite aufgrund kultureller Abgrenzung}

Der oben beschriebene Abgrenzungsprozess als Selbstschutz gegenüber der Systemumwelt wird in der systemtheoretischen Diskussion als Wahrnehmungsdefizit aufgrund dieser Abgrenzung beschrieben. Unreflektierte Reproduktion der offiziellen und inoffiziellen Regeln führt zu systemspezifischen Handlungen der Mitglieder eines Systems und ignoriert externe Impulse. Diese Regeln („heimlichen Lehrpläne“) selektieren potenzielle Mitglieder, z. B. die gewünschte Zielgruppe eines Studiengangs, und haben einen wesentlichen Einfluss auf die weitere Entwicklung, den Habitus, der Systemmitglieder. Man kann davon sprechen, dass sich die Ingenieurwissenschaften als System selbst in einem geschlossenen Prozess anhand der eigenen Einheit, Struktur und Kultur kontinuierlich selbst reproduzieren („Autopoiese“; vgl. Goorhuis 1996; Maturana 1993; Luhmann 1987). Gelangen externe Impulse als „Verstörungen“" in das System hinein, werden sie durch die Systemelemente so kompensiert, dass sich das System in seiner Kultur nicht verändern muss. ${ }^{1}$

Die Mitgliedschaft ist ein wesentliches Symbol für die erfolgreiche Identitätsentwicklung von Personen im System und im Sinne des Systems. Der Habitus, als Ergebnis dieser systemspezifischen Identitätsentwicklung drückt sich u.a. durch eine disziplinspezifische Sprach- und Denkstruktur, die weit über das eigentlich Fachliche hinausgeht, aus (vgl. Kosuch 1994: 56f; Bourdieu 1982: 174). Gelingt bei Mitgliedern des Systems die Ausbildung eines konformen Habitus, ist die Chance eines erfolgreichen Studienabschlusses deutlich höher, als bei Diskrepanzen zwischen Person und Rolle (Abbildung 2). Umgekehrt führt die formale Gleichbehandlung von unterschiedlichen Personen und Gruppen

1 Ausgangspunkt dieses Begriffes ist der von Maturana/Varela benutzte Begriff ,perturbacion“ (vgl. Maturana/Varela 1987: 27), der im Zusammenhang sozialer Phänomene mit „Verstörung“ übersetzt wird. 
(z. B. Frauen) dazu, dass ungleiche Chancen zur Habitusentwicklung geschaffen werden. Dies gilt für Schulen, Hochschulen, Unternehmen und Verbände (vgl. u.a. Ihsen 1999).

Diese (technische) Fachkultur ist ein virtuelles System mit festen Grenzen. ${ }^{2}$ Sie dient primär der Identität seiner Mitglieder und zur Selbsterhaltung des Faches. Eine solche Kultur produziert und vermittelt einen eigenen Sinn, indem verschiedene Leitideen ausgewählt werden (vgl. Rehberg 1992: 12) und damit innerhalb dieser Kultur gesetzesähnlich werden. Die Leitideen sind in Zielen, Strategien und Grundsätzen zu finden, wie auch in der Form der Machtverteilung, Führungsgrundsätzen, Funktionsrollen und „typischen Einstellungen“ (Abbildung 2).

So erklärt sich, dass sich u.a. auch Hochschulstrukturen durch die Fachkultur und den damit einhergehenden Habitus weiter fort reproduzieren, indem Regeln vermittelt werden, die ausschließlich Männern vorbehalten sind. Frauen sollen sich zwar der Fachkultur anpassen, dürfen aber nicht die gleichen Regeln für sich in Anspruch nehmen. Deutlich wird das $u$. a. durch die unterschiedlichen Konventionen und Spielregeln in der Kommunikation.

\section{Abbildung 2: „Leitkultur" und Diversity - ein Konflikt}

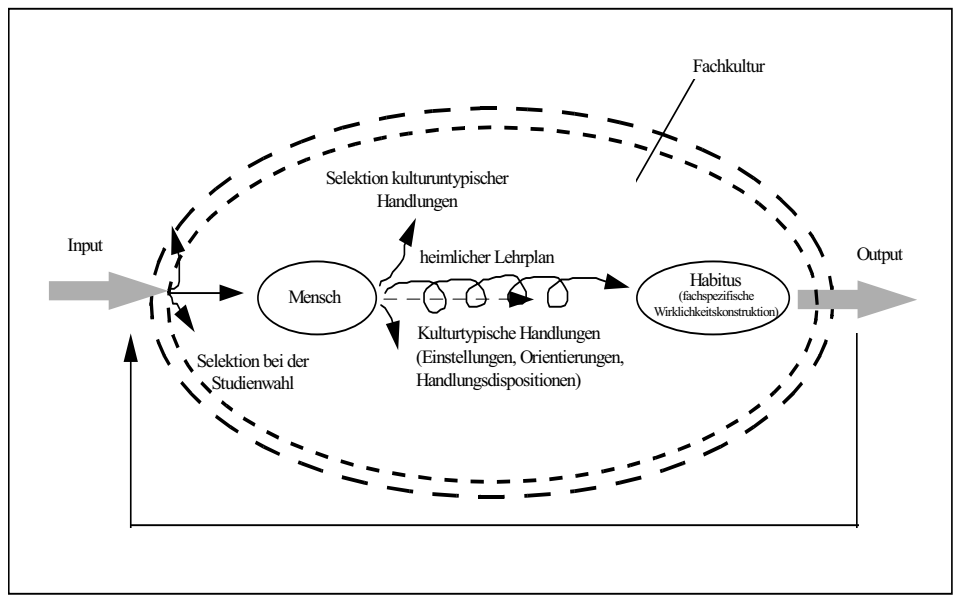

Quelle: Ihsen 1999

Insofern führt der begonnene Paradigmenwechsel in den Ingenieurwissenschaften (prozessorientiert, stärker interdisziplinär ausgerichtet) mög-

2 Ausführlich werden Theorie, Methode und Beispiele beschrieben in: Ihsen 1999. 
licherweise $\mathrm{zu}$ einer stärkeren Respektierung so genannter weiblicher Kompetenzen, allerdings erst dann, wenn sich der Wechsel auch auf eine Veränderung von Studien- und Berufsstrukturen auswirkt.

Der oben beschriebene Abgrenzungsprozess als Selbstschutz gegenüber der Systemumwelt wird in der systemtheoretischen Diskussion als Wahrnehmungsdefizit aufgrund dieser Abgrenzung beschrieben. Unreflektierte Reproduktion der offiziellen und inoffiziellen Regeln führt zu systemspezifischen Handlungen der Mitglieder eines Systems und ignoriert externe Impulse. Diese Regeln („heimlichen Lehrpläne“) selektieren potenzielle Mitglieder, z.B. die gewünschte Zielgruppe eines Studiengangs, und haben einen wesentlichen Einfluss auf die weitere persönliche Entwicklung, den Habitus, der Systemmitglieder (Abbildung 2).

Auf diese Weise gelingt es dem System, seine eigene Identität trotz stetig neuer Mitglieder zu schützen und bei diesen diejenigen Prozesse zu initiieren, die den eigenen, systemischen Interessen entsprechen (vgl. Goorhuis 1996: 144).

Studentinnen der Ingenieurwissenschaften, die von einem selbstverständlichen gleichberechtigten Umgang mit ihnen ausgehen, sammeln innerhalb dieses Systems in Praktika und Studium Erfahrungen darin, „aus dem Rahmen zu fallen“. Sie entwickeln Lösungsansätze, sich kulturell zu integrieren, können damit aber den Konflikt zwischen Geschlechts- und Berufsrolle nur begrenzen, nicht aufheben (vgl. Ihsen 1996; 2006). Da sie einen Teil der Fachkultur für sich adaptieren, entwickeln sie häufig zunächst individuelle Strategien (im Sinne von: „Ich bin schuld, wenn ich nicht akzeptiert werde, also kann ich es auch ändern“, vgl. Ihsen 1996), die dem allgemeinen ingenieurwissenschaftlichen Habitus entsprechen. Mit der Reflexionsleistung, dass auch noch so genaue Anpassung nicht zu der gewünschten Normalität führt, werden strukturelle Erklärungsansätze heran gezogen (im Sinne von: „Ich werde ausgegrenzt, weil ich eine Frau bin“, vgl. Ihsen 1996). Diese Erkenntnis kann zu verschiedenen Ergebnissen führen: zum Verlassen dieser Kultur, zu weiteren individuellen Anpassungsbemühungen, zur inneren Emigration innerhalb der Kultur und zur konstruktiven Auseinandersetzung mit dem System.

Die Fachkultur in den Ingenieurwissenschaften reproduziert ein Selbstverständnis, das auf sachlichen Problemlösungen durch spezielles Fachwissen und eine traditionelle Verwurzelung im industriell-ökonomischen Sektor beruht. Unberücksichtigt bleibt dabei, dass mit Technik und ihrer Gestaltung auch die Fantasie von Gestaltung, Schöpfung, 
Macht, Reinheit und Schönheit einhergeht. Das heißt, das Selbstverständnis von Ingenieuren und Ingenieurinnen und ihr Blick auf die Technik sind hochgradig emotional. Pikanterweise können diese Emotionen und die damit verbundenen Attribute auch auf den traditionellen Blick von Männern auf Frauen angewendet werden. Durch die historische und bildungspolitische Verschiebung der Beteiligung von Frauen in der Berufswelt konnte sich diese Gleichsetzung sehr lange unreflektiert halten und sich in der männlich dominierten Fachkultur verselbständigen. Frauen haben deshalb, ungeachtet der von ihnen erlangten Funktionen, nicht die Möglichkeit, sich vollständig ihrer eigenen Fachkultur anzupassen, können somit auch nicht „auf einem Auge blind“ werden, sondern stehen als mögliche Unterstützerinnen von Veränderungsprozessen zur Verfügung.

\section{Gender und Diversity-Management in Unternehmen}

Etliche Unternehmen in Deutschland haben sich längst auf den Weg gemacht, ihre Strukturen darauf hin zu überprüfen, ob sie Frauen und Männern gleiche Chancen bieten und was zu tun ist, um in den technischen Sparten mehr Frauen zu integrieren und weiter zu entwickeln. Dabei ist in Deutschland und Europa „Gender“ häufig der erste Schritt bei der Einführung eines Diversity-Managementkonzeptes, das mindestens noch die Generationengerechtigkeit und die Multikulturalität beinhalten.

Oberstes Ziel solcher Managementeinführungen ist es, keine Vorurteile und Diskriminierungen, sondern vollständige Integration aller Beschäftigtengruppen im Unternehmen zu erreichen. Dies führt zu einem unmittelbaren Perspektivenwechsel, weg vom Blick auf Mehrheiten und ihre Bedürfnisse hin zur Berücksichtigung von Frauen und Männern, Menschen unterschiedlicher Hautfarben, Nationalitäten und Herkunftskulturen, aus verschiedenen Generationen, versehen mit unterschiedlichen Kompetenzen.

In einer regelmäßig durchgeführten Untersuchung der DAX30notierten Unternehmen seit 2005 konnte festgestellt werden, dass inzwischen $75 \%$ dieser Unternehmen mit Leitsätzen, Konzepten, Programmen, Kooperationen und Beispielen an die Öffentlichkeit geht. Im Dezember 2006 unterzeichneten vier multinational agierende Konzerne unter der Schirmherrschaft von Bundeskanzlerin Merkel eine so genannte „Charta der Vielfalt“, mit der sie sich verpflichten, allein im Jahr 2007 150 weitere Unternehmen für das Thema „Diversity“ zu gewinnen. Da Unternehmen häufig erst dann die Öffentlichkeit suchen, wenn sie ihre 
neuen Prozesse gut und sicher im Griff haben, dürfen wir eine positive „Dunkelziffer" annehmen.

Oberstes Ziel solcher Managementeinführungen ist es, keine Vorurteile und Diskriminierungen, sondern vollständige Integration aller Beschäftigtengruppen im Unternehmen zu erreichen. Dies führt zu einem unmittelbaren Perspektivenwechsel, weg vom Blick auf Mehrheiten und ihre Bedürfnisse hin zur Berücksichtigung von Frauen und Männern, Menschen unterschiedlicher Hautfarben, Nationalitäten und Herkunftskulturen, aus verschiedenen Generationen, versehen mit unterschiedlichen Kompetenzen.

Die Einführung des Gesichtspunktes „Diversity“ erfolgt in Unternehmen mit erfolgreicher Umsetzung als „Top down“-Approach, wird also von der Unternehmensleitung als Unternehmensziel vorgegeben. Dem folgt unmittelbar eine Sensibilisierung aller Unternehmensbereiche und Mitarbeiter und Mitarbeiterinnen, warum bis wann welche Maßnahmen umgesetzt werden sollen. Diese sollten aus den Unternehmenszielen abzuleiten sein. Ziel der Sensibilisierung ist, alle Mitarbeiterinnen und Mitarbeiter in die Umsetzung von konkreten Diversity-Maßnahmen einzubinden, Ängste zu nehmen und dafür zu sorgen, dass trotz Veränderung die Identifikation mit dem Unternehmen erhalten bleibt bzw. noch zunimmt. Nach außen soll ein Diversity-Managementkonzept natürlich die besonders berücksichtigten Gruppen ansprechen und motivieren, sich im Unternehmen zu bewerben.

Argumente für die Einführung von Diversity-Management lassen sich drei Bereichen zuordnen:

- der betriebswirtschaftlichen Ebene: ein Unternehmen verspricht sich Wettbewerbsvorteile, insbesondere beim Recruiting, durch ein offenes, die oben angesprochenen Zielgruppen einbeziehendes Image und ein konstruktives Unternehmensklima durch motivierte Mitarbeiter und Mitarbeiterinnen

- der volkswirtschaftlichen Ebene: durch den demografischen Wandel und die bereits jetzt zu niedrigen Studierendenzahlen in ingenieurwissenschaftlichen Studiengängen entsteht ein zunehmender Fachkräftemangel in technischen Berufen, der sich Konjunktur gefährdend auswirken wird.

- der technischen Ebene: aufgrund neuer technischer Entwicklungen, z.B. in den Bereichen Optische Technologien, Mikrosystemtechnik, Nanotechnik und Energietechnik entstehen neue Märkte für neue Produkte. Ausgehend von der Erkenntnis, dass sich Produkte für eine stärkere Kundendiversifizierung entwickeln lassen, wenn auch 
die Entwicklungsteams vielfältiger zusammengesetzt sind, als bisher, können in den neuen Technikbereichen direkt DiversityMaßstäbe angelegt werden, um sich spätere „Nachbesserungen“ sparen zu können.

Gründe für Diversity im Unternehmensmanagement sind in der breiteren Personalbeschaffung, den Möglichkeiten einer intern verbesserten $\mathrm{Zu}$ sammenarbeit, der Marketingmöglichkeiten, und - nach nationalem Hintergrund - auch in der Antidiskriminierungs-Gesetzgebung zu finden.

\section{Zur Situation von Ingenieurinnen in Deutschland}

Trotz mannigfaltiger Maßnahmen, Programme und entsprechender Öffentlichkeitsarbeit seitens der Hochschulen, der Berufsverbände, Ministerien und Unternehmen geht die quantitative Entwicklung von Ingenieurinnen in Deutschland nur mühsam voran. Die Studierendenzahlen in den Ingenieurwissenschaften sind, trotz generell guter Berufsaussichten, zu niedrig; der Anteil der Studentinnen stagniert. Seit Jahren kommen wir über einen 10 \%igen Frauenanteil in der Berufsgruppe nicht hinaus; der Anteil der arbeitslos gemeldeten Ingenieurinnen liegt aktuell bei $18 \%$ (zum Vergleich: die Arbeitslosigkeit ihrer männlichen Kollegen liegt bei 7,2 \%, vgl. hierzu: www.vdi.de/monitoring).

Es ist sicherlich davon auszugehen, dass der oben beschriebene Veränderungsprozess parallel $\mathrm{zu}$ den bisherigen Selektionsverfahren verläuft, d.h. es werden sich deutlich mehr Unternehmen weiterhin schwer tun, Ingenieurinnen einzustellen und personalpolitisch zu fördern, als bislang Unternehmen mit der erklärten Absicht in die Öffentlichkeit getreten sind, die Fähigkeiten der Fachfrauen konsequent zu nutzen.

Und diese selbst haben klare Vorstellungen, wie sie leben und arbeiten wollen. Frauen lehnen eher als Männer eine eindimensionale Karriere ab. Sie wollen nicht ausschließlich arbeiten, sondern gleichzeitig ein Privatleben haben - ein Ansatz, der auch vielen Männern mindestens gesundheitlich ebenfalls gut anstünde (vgl. Kosuch 1994; VDI 2003; Ihsen 2006). Unternehmen, die es mit Gender und Diversity ernst nehmen, brauchen neue Arbeitszeitmodelle, neue Konzepte für Teilzeit-Karrieren, Angebote zur Unterstützung bei Betreuungsengpässen - sei es von einem Kind oder einem zu pflegenden Familienmitglied. 


\section{Fazit}

Die hier skizzierten und auf Nachhaltigkeit und Flexibilität ausgerichteten Veränderungsprozesse führen mittel- und langfristig zu einer kontinuierlichen Beteiligung von Frauen in den Ingenieurwissenschaften, wenn sie nicht tagepolitisch wirtschaftlichen Interessen zum Opfer fallen. Damit werden sich Unternehmen und Hochschulen verstärkt in interdisziplinärer Kooperation auf künftige Anforderungen hinsichtlich neuer Zielgruppen ausrichten können.

Der hier vorgestellte Ansatz von Gender Studies positioniert Ausbildung und Beruf in ihrer öffentlichen Wirkung neu: künftig wird es stärker als heute darum gehen, in welcher Weise die verschiedenen Zielgruppen von Gender- und Diversity-Maßnahmen unterschiedlich angesprochen werden müssen, um gezielt gefördert werden zu können. Auch in Deutschland werden sich Hochschulen und ingenieurwissenschaftliche Fachbereiche stärker als heute mit Diversity hinsichtlich des Geschlechts, des Alters, der Internationalität und einer zunehmenden Vielfalt der Studienabschlüsse auseinandersetzen müssen.

Es ist davon auszugehen, dass die Integration von Frauen in die technischen Berufe der entscheidende Maßstab für die Integration der anderen beiden Gruppen sein wird. Für kontinuierliche Berufstätigkeiten und Karrieren von Ingenieurinnen auf den verschiedenen Ebenen ihres Berufs und gleichzeitig für Elternzeit nehmende oder ihre Eltern pflegende Ingenieure braucht es bis zur Normalität noch etwas Zeit. Kontinuierliche Frauenkarrieren werden aber der entscheidende Dreh- und Angelpunkt für eine Steigerung der Studentinnenanteile in den ingenieurwissenschaftlichen Studiengängen sein. Dazu gehören sich langsam wandelnde Bilder: das des Ingenieurberufs genau so wie das der Gesellschaft hinsichtlich ihrer individuellen Freiheitsgrade bei der Übernahme von Verantwortung, egal welchem Geschlecht, welcher Generation oder welchem kulturellen Herkunft jemand angehört. 


\section{Literatur}

Bourdieu, Pierre (1982): Die feinen Unterschiede, Frankfurt/Main.

Brödner, Peter (1996): Innovationsstrategien im Wandel. Die Rolle der Ingenieure. In: Profil zeigen! Berufsstrategien für Ingenieurinnen und Ingenieure. Tagungsband. Chemnitz, hg.v. Verein Deutscher Ingenieure (VDI), TU Chemnitz-Zwickau.

Goorhuis, Henk (1996): Universitäre Weiterbildung im „neuen Kapitalismus“. In: Hochschulpublikation der ETH Zürich, Zürich, S.140ff.

Ihsen, Susanne (1996): Studentinnen an einer Technischen Hochschule. Zur Situation von Maschinenbau-Studentinnen an der RWTH Aachen. In: FORUM Frauenforschung - Vorträge aus fünf Jahre, hg.v. Dörte Münch/Elvi Thelen, Darmstadt, S.107-130.

Ihsen, Susanne (1999): Zur Entwicklung einer neuen Qualitätskultur in ingenieurwissenschaftlichen Studiengängen. Ein prozeßbegleitendes Interventionskonzept, VDI-Fortschritt-Berichte, Reihe 16, Nr. 112 , Düsseldorf.

Ihsen, Susanne (2006): Von der homogenen technischen Fachkultur zu Mixed Teams - Gender - Diversity. In: Hochschuldidaktik und Fachkulturen. Gender als didaktisches Prinzip, hg.v. Anne Dudeck/ Bettina Jansen-Schulz, Bielefeld.

Kosuch, Renate (1994): Beruflicher Alltag in Naturwissenschaft und Ingenieurwesen. Eine geschlechtervergleichende Untersuchung des Konflikterlebens in einer Männerdomäne, Weinheim.

Luhmann, Niklas (1987): Die Autopoiesis des Bewußtseins. In: Selbstthematisierung und Selbstzeugnis: Bekenntnis und Geständnis, hg.v. Aloys Hahn/Volker Kapp, Frankfurt/Main.

Maturana, Humberto/Varela, Francisco (1987): Der Baum der Erkenntnis, Bern/München.

Maturana, Humberto (1993): Das Konzept der Autopoiesis. In: Information Philosophie, 21. Jg., Heft 4, S.42ff.

Molvaer, Janitha/Stein, Kira (1994): Ingenieurin - warum nicht? Berufsbild und Berufsmotivation von zukünftigen Ingenieurinnen und Ingenieuren. Ein interkultureller Vergleich, Frankfurt a.M./New York.

Rehberg, Karl-Siegbert (1992): Universität als Institution. Vortrag an der RWTH Aachen.

Rieckmann, Heijo/Weissengruber, Peter H. (1990): Managing the Unmanagable? Oder: Lassen sich komplexe Systeme überhaupt noch steuern? Offenes Systemmanagement mit dem OSTO-Systemansatz. In: Management Development im Wandel, hg.v. Herbert Kraus et al., Wien, S.27-96. 
Technische Universität München (TUM) (2004): Die gender-gerechte Hochschule. Das Konzept der Technischen Universität München zur durchgängigen und nachhaltigen Frauenförderung, Frauenbüro der TUM 2004, München.

Verein Deutscher Ingenieure (VDI) (1995): Ingenieurausbildung im Umbruch, Empfehlungen des VDI für eine zukunftsorientierte Ingenieurqualifikation, Düsseldorf.

Verein Deutscher Ingenieure (VDI) (2003): Karriere für Ingenieurinnen, Düsseldorf.

Verein Deutscher Ingenieure (VDI) (2004): Stellungnahme zur Weiterentwicklung der Ingenieurausbildung in Deutschland, Düsseldorf. 



\section{Frauengesundheit in der medizinischen Versorgung}

Claudia HornBerg Und MichaEla WeISHOFF-HouBEN

In den letzten Jahren hat sich eine geschlechterspezifische Forschung etabliert, die verstärkt die biomedizinischen Risiken, die Unterschiede im Krankheitsspektrum von Frauen und Männern, aber auch Unterschiede zwischen Männern und Frauen in Bezug auf verhaltensbedingte Risiken, die Inanspruchnahme medizinischer Leistungen und die Medikamentenverordnung untersucht.

\section{Historische Entwicklungen}

Gesundheit stellt in allen Epochen und Kulturen ein zentrales Thema dar, das als Synonym für Harmonie, Kraft und Stärke steht. Krankheit symbolisiert im Unterscheid hierzu oftmals ein Ungleichgewicht und Schwäche oder wird auch als göttliche Strafe für fehlerhaftes Verhalten ausgelegt (Labisch 2000). In der frühen Neuzeit gab es einen durchgängigen Bezug zwischen Geschlecht und Körper bzw. Krankheit, der maßgeblich durch kulturelle Interpretationsmuster geprägt wurde (Labouvie 1998). In der vormodernen Gesellschaft gingen Juristen und Mediziner davon aus, dass bei Frauen eine spezifische (anatomische), durch krankhafte Veränderungen der weiblichen Geschlechtsorgane bedingte Disposition zum Wahnsinn vorliegt (Kaufmann 1996), während psychische Auffälligkeiten bei Männern als Kopfkrankheit diagnostiziert wurden (Ellerbrock 2002). Mit der Aufklärung wurde Gesundheit weniger religiös sondern mehr theoretisch-rational erklärt, jedoch blieb die enge Verknüpfung von Moral und Gesundheit bestehen. In der Diskussion um Sexualität und Reproduktion wurde die Frau als weibliche Abweichung 
von der Norm, die vom männlichen Körper repräsentiert wird, angesehen (Seidel 1998). Erst in der Moderne wurden die Vermeidung und Heilung von Krankheiten zunehmend verwissenschaftlicht und institutionalisiert: Es etablierte sich die Dienstleistungswirtschaft „Gesundheitswesen“ (Gerhardt 1993; Ellerbrock 2002), von der Frauen zunächst wenig profitierten, da ihnen die Versicherung in den im Zuge der Industrialisierung entstehenden Krankenkassen vorenthalten wurde. Zudem war es ihnen bis ins beginnende 20. Jahrhundert verwehrt, an der zunehmenden Professionalisierung zu partizipieren, da ihnen der Zugang zum Medizinstudium verschlossen blieb (Bleker/Schleiermacher 2000).

Die Frauengesundheitsbewegung der 1970er Jahre rückte die geschlechtsspezifische Gesundheit in den Blickpunkt und formulierte den Anspruch auf die Selbstbestimmung der Frauen über ihren Körper (Boston Women's Health Book Collective 1980). Zentrale Themen der Frauengesundheitsbewegung integrierten Aspekte von Sexualität und Reproduktion, Missbrauch, Gewalterfahrung, Depression und Sucht, aber auch weibliche Körpererfahrung sowie erste Überlegungen einer frauenspezifischen Gesundheitsförderung (Stahr et al. 1991).

Seit Mitte der 1980er Jahre ist es im Rahmen des transdisziplinären wissenschaftlichen Diskurses zur Gesundheit in Deutschland zu einer Neuorientierung gekommen. Die Grenzen der individuell-kurativ ausgerichteten Medizin zeigten sich deutlich und eine umfassendere Sichtweise von Gesundheit setzt sich durch (Hurrelmann/Laaser 1998). Mit dem Programm „Gesundheit 2000“ der Weltgesundheitsorganisation (WHO 1985), in dem als ein Ziel die Gesundheit von Frauen benannt ist, vollzieht sich der Wandel von der kurativen Medizin hin zu einem ganzheitlichen salutogenetischen Konzept. Für die Entwicklung der Frauengesundheitsforschung ist der Paradigmenwechsel vom naturwissenschaftlich orientierten Risikofaktorenmodell hin zum Lebensstilkonzept von größter Bedeutung (Abholz et al. 1982; Blaxter 1990). Während das Risikofaktorenkonzept vorrangig auf (verhaltensbedingte) Risikofaktoren fokussiert, rückt das Lebensstilkonzept den Zusammenhang von individuellen Lebensstilen und sozialen Bedingungen in der Lebenswelt in den Mittelpunkt.

Unter dem Einfluss der Ottawa-Charta zur Gesundheitsförderung wurden zunehmend Partizipationsprozesse in Gang gesetzt, die Frauen in der Übernahme von mehr Verantwortung und Vorsorge für die eigene Gesundheit unterstützen und sie in ihren Eigenkompetenzen und Autonomie stärken, um die Angebote des Versorgungssystems selbstverantwortlich nachfragen zu können. So wurden für die vom Europäischen Regionalbüro der WHO initiierte Initiative „Investing in Women's Health“ (WHO 1994; 1995) als Handlungsprioritäten zur Verbesserung 
der Gesundheit von Frauen unter anderem die Einführung frauenfreundlicher Kostenstrategien, die Unterstützung von Programmen zur Förderung gesunder Lebensweisen, die Bekämpfung von Gewalt gegen Frauen sowie die Verbesserungen der Arbeitsbedingungen von Frauen in Gesundheitsberufen benannt.

\section{Genderrelevanz in der Gesundheitsforschung}

1997 wurde durch die EU im Vertrag von Amsterdam (Artikel 2 und 3 Gleichstellung von Frauen und Männern) das Gender Mainstreaming als wesentlicher Schritt hin zu einer verstärkten gesellschaftlichen Sensibilisierung für geschlechtssensible Problemfelder festgeschrieben. Durch Gender Mainstreaming wird die Unterscheidung von sex und gender, dem biologischen und dem sozialen Geschlecht, unterstrichen und deutlich gemacht, dass Männer und Frauen soziale Kategorien sind. Für die Gesundheitsforschung bedeutet dies eine Akzentverschiebung hin zu einer geschlechtersensiblen Betrachtungsweise, die aber zurzeit in vielen Bereichen noch nicht adäquat umgesetzt wird. So hat beispielsweise Johnson (2003) festgestellt, dass nur in $33 \%$ der Studien über die Behandlung kardiovaskulärer Erkrankungen eine Geschlechterdifferenzierung vorgenommen wurde.

Der vom Bundesministerium für Familie, Senioren, Frauen und Jugend (BMFSFJ 2002) herausgegebene „Bericht zur gesundheitlichen Lage von Frauen in Deutschland" verweist deutlich auf den Umstand, dass Frauen sich in den klassischen Morbiditäts- und Mortalitätsrisiken grundlegend von den Männern unterscheiden. Frauen sind nicht nur „anders“ krank, sie zeigen auch ein anderes Gesundheitsverhalten als Männer und haben einen zum Teil eingeschränkten Zugang zu bestimmten Bereichen der Gesundheitsversorgung (BMFSFJ 2003; Maschewsky-Schneider et al. 2001). Obwohl Fortschritte in den medizinischen Behandlungsmöglichkeiten zu einer gestiegenen Lebenserwartung geführt haben (Klotz et al. 1998) und Frauen heute mit ca. 81 Jahren einen Überlebensvorteil von etwa sieben Jahren gegenüber den Männern haben (BMG 2002), sind sie nicht nur häufiger von psychosomatischen und psychischen Erkrankungen betroffen, sondern frequentieren auch medizinische Versorgungsangebote weitaus häufiger als Männer. Diese scheinbar widersprüchliche Situation beschreibt das „Geschlechterparadox" (Kolip 2003). Unter Berücksichtigung des von der Medizinsoziologin Lois Verbrugge (1990) erarbeiteten Erklärungsmodells können für die Geschlechterunterschiede in der gesundheitlichen Lage von Männern und Frauen fünf ineinander greifende Ebenen benannt werden: 
- Biomedizinische Risiken, die z.B. auf Unterschiede im Hormon- oder Immunstatus von Frauen und Männern zurückzuführen sind. So werden beispielsweise Schilddrüsenerkrankungen und Migräne unter anderem durch den weiblichen Hormonspiegel beeinflusst. Anatomisch bedingt treten Blasen- und Nierenbeckenentzündungen häufiger bei Frauen auf als bei Männern. Männer und Frauen reagieren unterschiedlich auf Alkohol und Kanzerogene im Tabakrauch, aber auch auf Opiate und Anästhetika (Kolip et al. 2005). Dass biologische Unterschiede das Krankheitsspektrum mitbestimmen, zeigt z.B. der hohe Anteil an Krebserkrankungen der weiblichen Geschlechtsorgane sowie das bei Männern erheblich höhere Risiko für HerzKreislauf-Erkrankungen bis zum Alter von etwa 55 Jahren (Maschewsky-Schneider 1997).

- Erworbene Risiken, die sich sowohl aus unterschiedlichen gesundheitsrelevanten Verhaltensweisen (z.B. Lebens- und Konsumstile, Inanspruchnahme von Vorsorgeleistungen) sowie aus Unterschieden in den Lebens- und Arbeitsbedingungen, dem sozialökologischen Lebensumfeld und der sozialen Lebenslage ergeben. So starben beispielsweise im Jahr 2001, bedingt durch gesundheitliches Risikoverhalten wie Alkoholkonsum und riskantes Verkehrsverhalten, im Alter von 30 bis 49 Jahren viermal so viele Männer durch äußere Ursachen. Im Jahr 2002 waren äußere Gewalteinwirkungen, Verletzungen oder alkoholbedingte Lebererkrankungen bei Männern dieser Altersgruppe die häufigsten Todesursachen (Kolip et al. 2005). Gesundheit konstituiert sich in einem Prozess zwischen dem Individuum, seinem Verhalten und der Umwelt und unterliegt insofern einer Vielzahl von Einflüssen, die für beide Geschlechter unterschiedlich sind. Frauen sind daher anderen Risiken als Männern ausgesetzt und gehen auch anders mit diesen um (Kolip 2003).

- Psychosoziale Risiken, die sämtliche Aspekte des Gesundheits- und Krankheitsverhaltens sowie den Umgang mit und die Bewertung von gesundheitlichen Beeinträchtigungen integrieren. Frauen schätzen ihren Gesundheitszustand im Vergleich zu den Männern subjektiv schlechter ein. Sie nehmen Beschwerden früher wahr als Männer, nehmen eher medizinische Hilfe in Anspruch und konsumieren im Vergleich zur Gruppe der Männer wesentlich häufiger Medikamente. Während für Männer die Leistungsfähigkeit und damit die Abwesenheit von gesundheitlichen Beschwerden der Maßstab ist, steht für Frauen das Wohlbefinden im Mittelpunkt, wenn sie ihre Gesundheit beurteilen (Maschewsky-Schneider 1997).

- Die subjektive Wahrnehmung von Symptomen, die Bereitschaft und die Art über gesundheitliches Befinden Auskunft zu geben, ist ge- 
prägt durch geschlechtsspezifische soziale Erfahrungen, die die unterschiedlichen Gesundheitskonzepte von Männern und Frauen erklären. Frauen berichten erfahrungsgemäß extrovertierter und bereitwilliger über Symptome und Befindlichkeiten, wobei offen bleiben muss, ob die geschlechtsspezifischen Unterschiede in der Wahrnehmung der Beschwerden oder in der Bereitschaft, darüber zu berichten, begründet sind (Kolip 2003). Im Bundesgesundheitssurvey berichteten im Jahr 1998 Frauen der Altersgruppe 30-65 Jahre häufiger über subjektive Beschwerden als Männer und gaben doppelt so häufig an, in den letzten sieben Tagen Kopf- oder Nackenschmerzen gehabt zu haben als Männer der gleichen Altersgruppe (Kolip et al. 2005).

- Auch die bereits erlebten Erfahrungen mit dem Gesundheitsversorgungssystem beeinflussen die Gesundheit von Männern und Frauen. Dass Frauen und Männer hier sehr unterschiedlich „behandelt“ werden, ist am Beispiel der koronaren Herzkrankheit zu verfolgen. Hier kommen nicht nur Unterschiede in der Diagnostik zum Tragen sondern auch in den Therapie- und Rehabilitationsoptionen, die Frauen und Männern angeboten werden (Biesig/Gutzwiller 2002).

Das Zusammenwirken dieser sich auch wechselseitig beeinflussenden Faktoren stellt ein äußerst komplexes Geschehen dar, das bisher nur unvollständig aufgeklärt ist (Kuhlmann 2002). Dennoch haben die verschiedenen Erklärungsebenen eine hohe Relevanz für die Weiterentwicklung der geschlechtersensiblen Gesundheitsforschung und -versorgung, da sie richtungweisende Anknüpfungspunkte bieten und den Blick auf die Frage richten, in welchen Bereichen die soziale Lebenswelt von Frauen und Männern zur Förderung von Gesundheit und der Gesundheitsversorgung verbessert werden muss (Maschewsky-Schneider 1997).

\section{Elemente einer frauengerechten Gesundheitsforschung und -versorgung}

Der Landtag NRW setzte im Jahr 2000 eine Enquetekommission zum Thema „Zukunft einer frauengerechten Gesundheitsversorgung in $N R W^{\text {" }}$ ein. Hintergrund war die Erkenntnis, dass es an einer geschlechtsspezifischen Betrachtung und Behandlung physischer und psychischer Erkrankungen noch mangelt. Die Kommission hatte den Auftrag, verschiedene Aspekte der Gesundheitsversorgung in NRW zu untersuchen und Handlungsempfehlungen für politische Entscheidungsorgane und das Gesundheitswesen zu erarbeiten. 
Vor diesem Hintergrund ist die Implementierung des Gender Mainstreaming als bedeutungsvolle Initiative hinsichtlich des Abbaus geschlechtsspezifischer Benachteiligung zu werten. Diese Aufgabe ist gerade unter gesundheitspolitischer Perspektive von größter Relevanz, da die Offenlegung der Entwicklungen und Versorgungsmängel für verschiedene soziale Gruppen einen zentralen Orientierungspunkt für gesundheits- und sozialpolitische Planungen und die Implementierung von Maßnahmen und entsprechenden Handlungsstrategien zur Förderung der Gesundheit darstellt (Vogt 1998; Kuhlmann 2002).

Die aktive Beteiligung und Einbeziehung von Frauen als Expertinnen ihrer eigenen Lebenslage im Gesundheitswesen könnte wesentlich dazu beitragen, Versorgungslücken und -defizite aufzudecken und einen engeren Bezug zwischen Lebensstil und Gesundheit in den Versorgungsangeboten herzustellen. Der Sachverständigenrat für die Konzertierte Aktion im Gesundheitswesen (2002) benennt diese Aufgabe als „Partizipative Patientinnenorientierung“. Beteiligung setzt jedoch das Bereitstellen und Verbreiten gesundheitsrelevanter Informationen voraus, die entsprechend den Bedürfnissen von Frauen aufbereitet und geeignet sind, die bestehende Informationsasymmetrien zwischen Frauen als Konsumentinnen von Gesundheitsleistungen und den Gesundheitsexperten zu verringern.

Aus allen bislang vorliegenden Analysen lässt sich die Notwendigkeit ableiten, das Gesundheitssystem geschlechtsspezifisch auszurichten, um auf diesem Wege den besonderen Bedürfnissen von Frauen gerecht werden zu können. Der für die Gesundheitsversorgung nahezu durchgängig festzustellende Gender-Bias, mit der Folge einer Über-, Unterund Fehlversorgung von Frauen in verschiedenen Bereichen des Gesundheitssystems, unterstreicht dieses Erfordernis. So starben nach dem Augsburger Herzinfarktregister im Jahr 2000 1,2 mal mehr Frauen als Männer in den ersten 28 Tagen nach einem Herzinfarkt, seit 2002 hat sich die 28-Tage-Letalität der Frauen der der Männer angeglichen (Kolip et al 2005). Die Übersterblichkeit der Frauen, vor allem in der Prähospitalphase, war vor allem dadurch bedingt, dass die bei Frauen eher auftretenden Begleitsymptome wie Übelkeit, Erbrechen, Schmerzen im Schulter- und Nackenbereich nicht frühzeitig als Herzerkrankung wahrgenommen wurden (Härtel 2002). Standards in der Diagnostik und Therapie von koronaren Herzkrankheiten sind an Männern orientiert, so dass den unterschiedlichen Verläufen der koronaren Herzkrankheit bei Männern und Frauen nur unzureichend Rechnung getragen werden kann. Die höhere Sterberate von Frauen nach einem Herzinfarkt wird als eine Konsequenz von Fehldiagnosen diskutiert, die zu einer verzögerten Behandlung führen (Kolip 2003). Für die weitere medizinische Versorgung ist 
von Bedeutung, dass Frauen nach einem Herzinfarkt nicht über so erfolgreiche Bewältigungsstrategien wie Männer verfügen, nicht so häufig an Rehabilitations-Maßnahmen teilnehmen und Rehabilitations-Maßnahmen häufiger abbrechen (Büchner et al. 2005). Eine andere Form der Fehlversorgung zeigt sich in den medizinisch nicht indizierten gynäkologischen Eingriffen und der Pathologisierung der Wechseljahre, die in der Medizin nahezu ausschließlich unter dem Aspekt der „Beschwerden“" als Legitimation für die Richtigkeit einer Hormonersatztherapie betrachtet werden (Kuhlmann 2002).

Defizite in der Gesundheitsversorgung sind nicht nur im Bereich der therapeutischen Interventionen, die Geschlechterfragen weitgehend ignorieren, festzustellen. Vergleichbare Analysen liegen für die Prävention und Gesundheitsförderung vor (Kolip 2003).

Voraussetzung für effektive Präventionsmaßnahmen und medizinische Versorgungsangebote ist zunächst eine entsprechend Datenbasis, die sich aus einer geschlechtsspezifisch differenzierenden Gesundheitsberichterstattung ableitet und Anhaltspunkte für Zielvorgaben zur Verfügung stellt. Die Gesundheitsversorgung für Frauen kann und wird es nicht geben, da Frauen keine homogene Gruppe darstellen und Angebote sich stets daran zu orientieren haben, um welche Frauen, in welchen speziellen Lebenssituationen es sich handelt.

Durch die zunehmende Institutionalisierung (z.B. Netzwerk Frauenforschung NRW, Einrichtung einer Professur für Frauengesundheit an der Charité Berlin) und Gremienarbeit (z.B. Bund-Länder-Kommission „Frauen in der Medizin“, Enquête-Kommission Frauengesundheit NRW), aber auch durch vielfältige Veranstaltungen zu den Themenbereichen „Geschlechterforschung in der Medizin - Frauen in der Medizin“ erhalten Wissenschaftlerinnen ein Forum, um sich und ihre Forschungsarbeiten zur differenzierten Betrachtung von Krankheit und Gesundheit bei Männern und Frauen vorzustellen und auf die Notwendigkeit einer geschlechtssensiblen Forschung aufmerksam zu machen. Andererseits werden Defizite in der Gesundheitsforschung sowie in der Information über die Auswirkungen von Lebenswelten auf die gesundheitliche Situation und Versorgung fokussiert. Dies gilt es insbesondere in einer Zeit zu sichern, die Gefahr läuft, von einem stringenten Ökonomismus geprägt zu werden.

„Mainstreaming“ als tragendes gesundheits- und sozialpolitisches Prinzip beinhaltet in diesem Kontext die Forderung, zielgruppen- und problemorientierte Angebote mit niedriger Zugangsschwelle zu fördern, um ungleiche Gesundheitschancen zwischen den Geschlechtern und sozialen Schichten abzubauen, um damit die Voraussetzung zu schaffen, allen Bevölkerungsgruppen eine bessere Teilhabe am Gesundheitssys- 
tem $\mathrm{zu}$ ermöglichen und so die Gesundheit von Frauen und Männern nachhaltig zu verbessern. Darüber hinaus ist anzuregen, die bestehende Indikatorensammlung der Gesundheitsbefragung um umweltbezogene, genderrelevante Gesundheitsindikatoren zu erweitern, damit Datenerhebungen bzw. -auswertungen entsprechend nach Geschlechtern getrennt vorgenommen werden können (Pauli/Hornberg 2006). Wichtig ist in dem Zusammenhang auch, die sozialen, ethnischen, altersspezifischen etc. Unterschiede im Hinblick auf die Identifikation empfindlicher Personengruppen zu berücksichtigen.

Analysen und Bewertungen im Kontext Gender, Umwelt und Gesundheit müssen unter Berücksichtigung aller gesundheitsrelevanten Einflussfaktoren (Bargfrede et al. 2004) erfolgen. Hier sind insbesondere Unterschiede in den Lebensstilen, den sozioökonomischen Lebensverhältnissen (Mielck 2002; Bolte/Mielck 2004) sowie der Wohnregion (Arend 1998; Maschewsky 2001; Mielck/Heinrich 2002) und den Arbeitsverhältnissen (BFS 1997; Keller et al. 2005) zu berücksichtigen. Dies ist umso wichtiger, da sich im Lebensverlauf in Abhängigkeit von den Lebensbedingungen Expositionsfaktoren verändern. Hinzu kommen unterschiedliche Suszeptibilitätscharakteristika von Männern und Frauen, welche die individuelle Empfindlichkeit gegenüber Schadstoffen determinieren (Stopper/Gertler 2002). Sowohl in der Exposition gegenüber Umweltbelastungen als auch in den umweltbezogenen, d.h. durch Umweltfaktoren mit beeinflussten gesundheitlichen Beschwerden (Hornberg et al. 2005) werden Geschlechterunterschiede sichtbar (Keller 2004; Keller et al 2004; 2005). In diesem Kontext bedarf es der Zusammenarbeit gendersensibler medizinischer Fachdisziplinen, um zugrunde liegende Ursachen adäquat abzuklären (Hornberg et al 2003; Huss et al 2004) und beratende, expositionsmindernde und/oder medizinisch-therapeutische Maßnahmen einleiten zu können.

\section{Literatur}

Abholz, Heinz-Harald/Borgers, Dieter/Karmaus, Wilfried/Korporal, Johannes (Hg.) (1982): Risikofaktorenmedizin, Berlin.

Arend, Michael (1998): Sozialfall Lärm. Vom Lärm sind wir alle betroffen, aber nicht alle gleich. In: Cerclebruit: Lärm. Luzern/Basel. http://www.cerclebruit.ch,12.06.05.

Bargfrede, Anja/Pauli, Andrea/Hornberg, Claudia (2004): Gesundheit: Zur gesundheitlichen Situation von Frauen. In: Handbuch Frauenund Geschlechterforschung, hg.v. Ruth Becker/Beate Kortendiek, Frankfurt am Main, S. 519-528. 
Biesig, Brigitte/Gutzwiller, Felix (Hg.) (2002): Frau und Herz: Epidemiologie, Prävention und Behandlung der koronaren Herzkrankheit bei Frauen in der Schweiz, Bern/Göttingen/Toronto/Seattle.

BFS (Bundesamt für Statistik) (1997): Berufspezifische Mortalitätsrisiken der Männer in der Schweiz, Bern.

Blaxter, Mildred (1990): Health and Lifestyles, London.

Bleker, Johanna/Schleiermacher, Sabine (2000): Ärztinnen aus dem Kaiserreich. Lebensläufe einer Generation, Weinheim.

Bolte, Gabriele/Mielck, Andreas (Hg.) (2004): Umweltgerechtigkeit. Die soziale Verteilung von Umweltbelastungen, Weinheim/München.

Boston Women's Health Book Collective (1980): Our bodies, ourselves. 1. und 2. Handbuch von Frauen für Frauen, Reinbek/Hamburg.

Büchner, Birgit/Kleiber, Christina/Stanske, Beate/Hermann-Lingen, Christoph (2005): Stress und Herzkrankheiten bei Frauen. Geschlechtssprezifische Risiken, Bewältigungsprobleme und Behandlungsansätze, S. 416-428.

BMFSFJ (Bundesministerium für Familie, Senioren, Frauen und Jugend) (2002): Untersuchung zur gesundheitlichen Situation von Frauen in Deutschland. Eine Bestandsaufnahme unter Berücksichtigung der unterschiedlichen Entwicklung in West- und Ostdeutschland, Stuttgart.

BMFSFJ (Bundesministerium für Familie, Senioren, Frauen und Jugend) (2003): Broschüre Gender Mainstreaming. Was ist das?, Bonn.

Bundesministerium für Gesundheit (BMG) (2002): Statistisches Taschenbuch. Gesundheit 2002, Bonn.

Ellerbrock, Dagmar (2002): Geschlecht, Gesundheit und Krankheit in historische Perspektive. In: Geschlecht, Gesundheit und Krankheit: Männer und Frauen im Vergleich, hg.v. Klaus Hurrelmann/Petra Kolip, Bern, S. 118-141.

Gerhardt, Uta (1993): Gesundheit - ein Alltagsphänomen. Konsequenzen für Theorie und Methodologie von Public Health, hg.v. Wissenschaftszentrum Berlin für Sozialforschung, Berlin, S. 93-206.

Härtel, Ursula (2002): Krankheiten des Herz-Kreislauf-Systems bei Männern und Frauen. In: Geschlecht, Gesundheit und Krankheit. Männer und Frauen im Vergleich, hg.v. Klaus Hurrelmann/Petra Kolip, Bern, S. 273-290.

Hornberg, Claudia/Pauli, Andrea/Wiesmüller, Gerhard Andreas (2003): Multiple Chemical Sensitivity (MCS) - eine Herausforderung für die interdisziplinäre Patientenversorgung und Forschung. In: MedizinUmwelt-Gesellschaft, 16 (4), S. 274-285. 
Hornberg, Claudia/Malsch, Anette/Fides, Konstanze/Pauli, Andrea/ Weißbach, Wiebke/Wiesmüller, Gerhard Andreas (2005): Situationsbericht klinische Umweltmedizin Beispiel Nordrhein-Westfalen (NRW). In: Arbeitsmed.Sozialmed.Umweltmed., 40 (1), S. 12-26.

Hurrelmann, Klaus/Laaser, Ulrich (1998): Entwicklung und Perspektiven der Gesundheitswissenschaften. In: Gesundheitswissenschaften: Handbuch für Lehre, Forschung und Praxis, hg.v. Klaus Hurrelmann/Ulrich Laaser, Weinheim, S. 17-45.

Huss Anke/Küchenhoff, Joachim/Bircher, Andreas/Heller, Pia/Kuster, Hans/Niederer, Markus/Scartazzini, Grigor/Schwarzenbacher, Simone/Waeberg, Roger/Wegmann, Lukas/Braun-Fahrländer, Charlotte (2004): Symptoms attributed to the environment - a systematic, interdisciplinary assessment. In: Int J Hyg Env Health, 207 (3), S. 245-254.

Kaufmann, Doris (1996): Wahnsinn und Geschlecht. In: Was sind Frauen? Was sind Männer? Geschlechterkonstruktionen im historischen Wandel, hg.v. Christiane Eifert, Frankfurt am Main, S. 176-196.

Keller, Doris (2004): Geschlechterspezifische Betrachtung von Patienten der ehemaligen Umweltmedizinischen Beratungsstelle (UMEB) des Medizinischen Institutes für Umwelthygiene der Heinrich-HeineUniversität Düsseldorf. Dissertation zur Erlangung des akademischen Grades eines Doktors der Medizin, Aachen.

Keller, Doris/Hornberg, Claudia/Niggemann, Hiltrud/Neuhann, Heribert Florian/Ranft, Ulrich/Dott, Wolfgang/Wiesmüller, Gerhard Andreas (2004): Geschlechterspezifische Aspekte in der Umweltmedizin. In: Journal Netzwerk Frauenforschung NRW, 17, S. 25-38.

Keller, Doris/Hornberg, Claudia/Niggemann, Hiltrud/Neuhann, Heribert Florian/Ranft, Ulrich/Dott, Wolfgang/Wiesmüller, Gerhard Andreas (2005): Geschlechterassoziierte Expositionen bei Patienten einer umweltmedizinischen Beratungsstelle. In: Arbeitsmed.Sozialmed. Umweltmed., 40 (6), S. 342-353.

Klotz, Theodor/Hurrelmann, Klaus/Eickenberg, Hans-Udo (1998): Der frühe Tod des starken Geschlechts. In: Deutsches Ärzteblatt 95, H. 9, S. 460-464.

Kolip, Petra (2003): Frauen und Männer. In: Das Public Health Buch. Gesundheit und Gesundheitswesen, hg.v. Ulla Walter et al., München/Jena, S. 642-653.

Kolip, Petra/Lademann, Juli/Deitermann, Bernhilde/Bucksch, Jens/ Schwarze, Monica (2005): Schwerpunktbericht Gesundheit von Frauen und Männern im mittleren Lebensalter. Gesundheitsberichterstattung des Bundes, hg.v. Robert Koch-Institut Berlin. 
Kuhlmann, Ellen (2002): Statement zum Themenkreis II: Geschlechtsspezifische Gesundheitsforschung und Public Health. Fachtagung „Frauen in der Medizin“ des Arbeitskreises „Förderung von Frauen in der Medizin“ der Bund-Länder-Kommission, 7. November 2002, Bonn. www.zes.uni-bremen.de/ kuhlmann.

Labisch, Alfons (2000): Gesundheit im Wandel der Zeiten. Zur Geschichte und Theorie des Problems "Medizin in der Gesellschaft". In: Gesundheit: Strukturen und Handlungsfelder, hg.v. Bundesvereinigung für Gesundheit e.V., Neuwied, S. 1-49.

Labouvie, Eva (1998): Andere Umstände. Eine Kulturgeschichte der Geburt, Köln.

Maschewsky, Werner (2001): Umweltgerechtigkeit, Public Health und soziale Stadt, Frankfurt am Main.

Maschewsky-Schneider, Ulrike (1997): Frauen sind anders krank: Zur gesundheitlichen Lage der Frauen in Deutschland, Weinheim/München.

Maschewsky-Schneider, Ulrike/Hellbernd, Hildegard/Schaal, Werner/ Urbschat, Iris/Wieners, Karin (2001): Über-, Unter-, Fehlversorgung und Frauengesundheit. Ein Forschungsgegenstand für Public Health. In: Bundesgesundheitsblatt 44, S. 771-779.

Mielck, Andreas (2002): Soziale Ungleichheit und Gesundheit. In: Geschlecht, Gesundheit, Krankheit. Männer und Frauen im Vergleich, hg.v. Klaus Hurrelmann/Petra Kolip, Bern/Göttingen/Toronto/Seattle, S. 387-402.

Mielck, Andreas/Heinrich, Joachim (2002): Soziale Ungleichheit und die Verteilung umweltbezogener Expositionen (environmental justice). In: Gesundheitswesen, 64 (7), S. 405-416.

Pauli, Andrea/Hornberg, Claudia (2006): Eine gesunde und sichere natürliche Umwelt. In: Gender-Gesundheitsbericht Schweiz 2006, hg.v. Bundesamt für Gesundheit BAG, S.153-162.

Sachverständigenrat für die Konzertierte Aktion im Gesundheitswesen (2002): Bedarfsgerechtigkeit und Wirtschaftlichkeit, Baden-Baden.

Seidel, Hans-Christoph (1998): Eine neue „Kultur des Gebärens “. Die Medikalisierung von Geburt im 18. und 19. Jahrhundert in Deutschland, Stuttgart.

Stahr, Ingeborg/Jungk, Sabine/Schulz, Elke (1991): Frauengesundheitsbildung. Grundlagen und Konzepte, Weinheim/München.

Stopper Helga/Gertler, Martin (2002): Physikalische Umwelt und Gesundheit. In: Geschlecht, Gesundheit und Krankheit. Männer und Frauen im Vergleich, hg.v. Klaus Hurrelmann/Petra Kolip, Bern/ Göttingen/Toronto/Seattle, S. 439-459. 
Verbrugge, Louis M (1990): Pathways of Health and Death. In: Women, Health and Medicine in America. A Historical Handbook, hg.v. Rima D. Apple, New Brunswick/New Jersey, S. 41-79.

Vogt, Irmgard (1998): Standortbestimmung der Frauengesundheitsforschung. In: Frauen und Gesundheit(en) in Wissenschaft, Praxis und Politik, hg.v. Arbeitskreis Frauen und Gesundheit im Norddeutschen Forschungsverbund Public Health, Bern/Göttingen/Toronto/Seattle, S. 22-33.

WHO Weltgesundheitsorganisation (1985): Einzelziele für „, Gesundheit 2000 “, Kopenhagen.

WHO Regional Office (1994): Vienna Statement on Investing in Women's Health in the Countries of Central and Eastern Europe, Wien.

WHO Regional Office (1995): Investing in Women's Health in the Countries of Central and Eastern Europe, WHO Regional Publications, European Series, Nr. 55. 


\section{Die Autorinnen und Autoren}

Bessenrodt-Weberpals, Monika, Prof. Prof. Dr., Professur Gender und Naturwissenschaften, Hamburg.

Monika Bessenrodt-Weberpals schloss 1980 ihr Physikstudium mit dem Diplom an der Universität Düsseldorf ab, wo sie bis 1987 als wissenschaftliche Mitarbeiterin im SFB Plasmaphysik tätig war. Ihre erste Philologische Staatsprüfung für das Lehramt an Gymnasien in den Fächern Physik und Mathematik, sowie Philosophie und Pädagogik schloss sie 1981 ab und promovierte 1984 in Physik.

Sie arbeitet an der HAW Hamburg als Professorin für Gender und Naturwissenschaften und an der Universität Düsseldorf als außerplanmäßige Professorin für Physik.

Seit 1988 ist sie Mitglied in der Deutschen Physikalischen Gesellschaft (DPG) und war von 1995 bis 1997 Mitglied im Arbeitsausschuss des Fachverbandes Plasmaphysik der DPG. Weiter gehörte sie 1998 zu den Gründungsmitgliedern des Arbeitskreises Chancengleichheit (AKC) und fungiert dort seit 2000 als Sprecherin. Außerdem ist sie als Mitglied des Vorstandsrates der verantwortlichen DPG aktiv. Ihre Schwerpunkte liegen im Bereich Physik und Gender, insbesondere in der geschlechtergerechten Didaktik der Physik, Dual Career Couples und im Work-LifeBalancing im internationalen Vergleich. Das von ihr erarbeitete Konzept des kontextorientiertes Lehren und Lernen in Physik und Mathematik belebt sie mit aktivierenden Elementen.

Bessing, Nina, Projektleiterin an der Europäische Akademie für Frauen in Politik und Wirtschaft, Berlin.

Nina Bessing ist Projektleiterin und Trainerin in der Europäischen Akademie für Frauen in Politik und Wirtschaft (EAF). Sie hat Volkswirtschafts-, Betriebswirtschaftslehre und Erziehungswissenschaften 
studiert und ist seit 2002 für die EAF in verschiedenen Projekten tätig gewesen. Von 2004 bis 2006 war sie seitens der EAF zuständige Projektleiterin im Forschungsprojekt „Gender-Aspekte in der Forschung“.

Nina Bessing promoviert am Institut für Management bei Prof. Dr. Gertraude Krell an der Freien Universität Berlin zum Thema „Der Beitrag von Diversity zur Innovationsfähigkeit von Unternehmen“. Als Trainerin sind ihre Spezialgebiete: Bewerbungs- und Karrieretrainings, Work-Life-Balance für Führungskräfte, Gender und Diversity. Sie lebt mit ihrem Partner und ihrem Sohn in Berlin.

Bosch, Mineke, Prof. Dr., Director of Center and Diversity, Universität Maastricht.

Mineke Bosch ist Direktorin und außerordentliche Professorin am Center for Gender and Diversity der Universität Maastricht.

Sie studierte Geschichte mit dem Schwerpunkt Gender. Ihre Dissertation war eine Studie über Frauen und Gender in der höheren Bildung und den Naturwissenschaften in den Niederlanden zwischen 1872 und 1948.

Ihr geschichtliches Interesse an dem Problem „Frauen und Naturwissenschaft", lenkte ihre Aufmerksamkeit auf das zeitgenössische Verfahren und die politischen Maßnahmen in diesem Bereich. Sie war Mitglied der ETAN (Expertinnen Gruppe für Frauen und Naturwissenschaft) und Mitverfasserin, des Berichts der Europäischen Kommission ,Science Policies in the European Union: Promoting Excellence through Mainstreaming Gender equality“. Sie leitete das erste und ist in das zweite EQUAL-Projekt miteinbezogen, dass das Center for Gender and Diversity für Frauen und Naturwissenschaft ausführte. Sie gehört außerdem zu dem Vorstand der European Platform of Women Scientists.

Fisher, Allan, Ao. Prof. Dr., School of Computer Science, Carnegie Mellon University, Pittsburgh.

Allan Fisher ist Präsident und CEO der iCarnegie Inc., einem SpinOff der Carnegie Mellon University, USA.

Er studierte Chemie an der Princeton University sowie an der University of Cambridge und promovierte in Informatik an der Carnegie Mellon University. Er ist außerordentlicher Professor an der School of Computer Science, Carnegie Mellon University, und Mitglied zahlreicher Beratungsgremien im Bereich Diversity und Technik, u.a. am „Anita Borg Institute“ und im „National Research Council Committee on Women in Science and Engineering“"

Zusammen mit Dr. Jane Margolis - Sozialwissenschaftlerin und Expertin für Genderfragen bei Bildung - hat er an der School of Computer 
Science ein Studienförderungsprogramm ins Leben gerufen, das innerhalb von fünf Jahren einen Anstieg der Studienanfängerinnen in Informatik von $7 \%$ auf $42 \%$ bewirkte.

Hornberg, Claudia, Univ.-Prof. Dr. med. Dipl.-Biol. Dipl.-Ökol., Professorin für Biologie und Ökologie in den Gesundheitswissenschaften, Universität Bielefeld.

Claudia Hornberg studierte Biologie an der Ruhr-Universität Bochum und danach Ökologie an der Universität GH Essen. Das anschließende Medizinstudium absolvierte sie an der Heinrich Heine-Universität Düsseldorf. Ihre Dissertation schrieb Claudia Hornberg an der Medizinischen Fakultät der Universität Düsseldorf über Tracheobronchiale Epithelzellen als inhalationstoxikologisches In-vitro-Modell zur Analyse der Zytotoxizität und Genotoxizität von komplexen atmosphärischen Feinstäuben.

Die Fachärztin für Hygiene und Umweltmedizin ist an den Universitätskliniken Münster und Düsseldorf in den Bereichen Umweltmedizin und Infektiologie ärztlich tätig. Seit 2001 ist sie Universitätsprofessorin (C4) für das Fach „Biologie und Ökologie in den Gesundheitswissenschaften unter besonderer Berücksichtigung geschlechtsspezifischer Aspekte“ ernannt und seitdem Leiterin der Arbeitsgruppe „Umwelt und Gesundheit“" an der Fakultät für Geisteswissenschaften.

Ihsen, Susanne, Prof. Dr., Professorin für Gender Studies in den Ingenieurswissenschaften, TU München.

Susanne Ihsen, geboren 1964, studierte in Duisburg und Aachen Sozialwissenschaften und war dann als wissenschaftliche Mitarbeiterin am Hochschuldidaktischen Zentrum (heute: Zentrum für Lern- und Wissensmanagement) und am Lehrstuhl für Informatik im Maschinenbau an der RWTH tätig. Sie promovierte 1999 an der RWTH Aachen über die Entwicklung einer neuen Qualitätskultur in ingenieurwissenchaftlichen Studiengängen. Von 1999 bis 2004 war sie in der Hauptgeschäftsstelle des VDI (Verein Deutscher Ingenieure) beschäftigt und leitete dort vier Jahre die Abteilung Beruf und Karriere. Ihre Arbeitsschwerpunkte waren der Berufseinstieg, die berufliche Entwicklung von Ingenieurinnen sowie der Auf- und Ausbau einer Karriereberatung für VDI-Mitglieder. Im Dezember 2004 wurde sie auf die erste Professur für Gender Studies in Ingenieurwissenschaften an die Technische Universität München berufen. Ihre Themenschwerpunkte sind die Entwicklung eines modernen, gleichberechtigten, Berufsbildes, Diversity in der Technikentwicklung und -gestaltung sowie die Nachhaltigkeit von Maßnahmen zur Motivation von Schülerinnen und der Karriereentwicklung von Ingenieurinnen. 
Klees-Möller, Renate, Dr. phil., Zentrum für Hochschul- und Qualitätsentwicklung, Universität Duisburg-Essen.

Renate Klees-Möller ist promovierte Erziehungswissenschaftlerin. Seit mehr als zwanzig Jahren ist sie in der Frauen- und Geschlechterforschung mit den Schwerpunkten Mädchen und Frauen in der Sozialen Arbeit, Mädchensozialisation, Mädchen- und Frauenbildung, Beratung/ Mentoring für Frauen tätig. Weiterhin verfasste sie zahlreiche Veröffentlichungen zu diesen Themen.

Renate Klees-Möller ist in der Erziehungswissenschaftlichen Lehre an der Universität Dortmund und seit 2000 an der Universität DuisburgEssen tätig. Sie ist außerdem zuständig für die Konzeption und Umsetzung von Frauenförderungsprogrammen in der Wissenschaft. Seit 2001 war sie maßgeblich am Aufbau des Projektzentrums Meduse an der Universität Essen involviert. Seit leitet sie den Geschäftsbereichs Frauenförderung/Gender Mainstreaming am Zentrum für Hochschul- und Qualitätsentwicklung der Universität Duisburg-Essen. Viele Veröffentlichungen schrieb sie zur Mädchen- und Frauenbildung/-politik.

Leicht-Scholten, Carmen, Dr., Projektleitung der Mentoring-Programme, RWTH Aachen

Carmen Leicht-Scholten ist promovierte Sozialwissenschaftlerin. Nach einer Ausbildung als Übersetzerin schloss sie 1993 das Studium der Politischen Wissenschaften, Soziologie und Romanistik in Heidelberg, Aachen und Sevilla ab und promovierte an der Universität Hamburg am Lehrstuhl Politische Wissenschaft über die Wirkung der Verfassungsrechtssprechung auf Politik und Gesellschaft. In ihrer 1998 erschienenen Dissertation verknüpfte sie den feministischen Theoriediskurses mit den policy-orientierten Äußerungen der Bundesregierungen und maß die Rechtsprechung des BVerfG an diesen Parametern. Von 1998 bis 2004 arbeitete sie freiberuflich als Referentin und Dozentin in der Beratung und politischen Bildung, unter anderem als freie Lehrbeauftragte an diversen Hochschulen. Seit 2004 arbeitet sie als wissenschaftliche Assistentin am Institut für Soziologie der RWTH Aachen. Ihre Forschungsschwerpunkte sind Wissenschaft und Geschlecht, Chancengleichheit und Qualitätsentwicklung an Hochschulen, Geschlechtergerechte Konzepte der Personalentwicklung sowie Recht und Geschlecht. Sie ist wissenschaftliche Leiterin der Mentoring Programme TANDEM, des hochschulübergreifenden Mentoring Programms TANDEMplus und TANDEMplusMED, sowie des EU Projektes TANDEMplus IDEA. Seit Januar 2007 vertritt sie die RWTH Aachen in der WiST II (Women in Science and Engineering) Arbeitsgruppe der Europäischen Kommission. 
Lukoschat, Helga, Dr., Geschäftsführerin der EAF und der Femtec, Berlin.

Helga Lukoschat ist 1957 geboren und studierte Germanistik, Politikwissenschaft und Geschichte in Erlangen und Berlin. Sie war langjährig als Journalistin und Publizistin tätig, u.a. als Redakteurin der tageszeitung und als Referentin für Bündnis 90/Die Grünen in Berlin. Seit 1992 hat sie an mehreren Forschungsprojekten der Technischen Universität Berlin im Bereich Frauenforschung mitgearbeitet und mit einer Studie über Frauen in Führungspositionen promoviert.

Helga Lukoschat ist Geschäftsführerin der EAF und der Femtec. Hochschulkarrierezentrum für Frauen Berlin GmbH. Sie ist Mitbegründerin der EAF. Von 1997 bis 1999 war sie verantwortlich für das internationale mehrfach ausgezeichnete Mentoring-Programm „Preparing Women to Lead“. Frau Lukoschat ist verheiratet und lebt mit ihrem Partner in Berlin.

Margolis, Jane, Dr., School of Computer Science, Carnegie Mellon University, Pittsburgh.

Jane Margolis ist Sozialwissenschaftlerin an der University of California, Los Angeles mit dem Schwerpunkt soziale Ungleichheit im Bildungswesen. Seit 14 Jahren beschäftigt sie sich schwerpunktmäßig mit dem Zusammenhang von Gender, Rasse und Informatik. Sie ist Koautorin des, mit einem Preis ausgezeichneten, Buches Unlocking the Clubhouse: Women in Computing. Von 2000 bis 2004 war sie wissenschaftliche Leiterin einer, von der National Science Foundation geförderten, Studie mit der Untersuchung der Frage, warum so wenig afroamerikanische Männer und Frauen, so wenig lateinamerikanische Männer und Frauen und so wenig amerikanische Frauen Informatik an der Universität studieren.

Aktuell arbeitet sie an einem Buch zu diesem Thema, welches 2008 veröffentlicht werden soll.

Onnen-Isemann, Corinna, PD Dr. rer. pol. habil. Dipl. Soz., Professurvertreterin für Allgemeine Soziologie an der RWTH Aachen.

Corinna Onnen-Isemann ist promovierte Sozialwissenschaftlerin. Sie arbeitete als wissenschaftliche Angestellte in diversen empirischen Forschungsprojekten an der Universität Oldenburg, wo sie auch zum Dr. rer. pol. promovierte. Im Jahre 1999 erhielt sie für ihre Habilitationsschrift „Wenn der Familienbildungsprozeß stockt.... Eine empirische Studie über Stress und Coping-Strategien reproduktionsmedizinisch behandelter Partner“, ihre Venia legendi für Soziologie. 
Sie war Professurvertreterin am Lehrstuhl „Mikrosoziologie“ an der Humboldt Universität zu Berlin (2001) und für „Familie, Demographischer Wandel, Sozialstruktur, Internationale Migration“ an der Friedrich-Alexander-Universität Erlangen-Nürnberg (2002/03). Von 20032005 war sie Universitätsprofessorin für Gender Studies an der Universität Regensburg, seit WS 2006/07 vertritt sie die Professur für Allgemeine Soziologie an der RWTH Aachen.

Von 1997-2000 war Frau Onnen-Isemann Mit-Herausgeberin der Zeitschrift für Deutsches und Europäisches Familienrecht (DEuFamR).

Rompeltien, Bärbel, Dr., Zentrum für Hochschul- und Qualitätsentwicklung, Universität Duisburg-Essen.

Bärbel Rompeltien ist Literatur- und Sozialwissenschaftlerin. Sie übt eine langjährige Tätigkeit als Beraterin für Studierende aus und ist außerdem Autorin zu den Themen Beruf und Karriere, Kommunikation, Gleichstellung und Hochschulentwicklung. Frau Rompeltien war fünf Jahre Gleichstellungsbeauftragte, zunächst an der Universität Essen, dann in der Fusionsphase der Universität Duisburg-Essen. Sie ist Initiatorin des Projektzentrums MEDUSE, jetzt Zentrum für Hochschul- und Qualitätsentwicklung, Geschäftsbereich Frauenförderung/Gender Mainstreaming.

Schinzel, Britta, Prof. Dr., Professorin für Informatik und Gesellschaft, Universität Freiburg.

Britta Schinzel studierte Mathematik, Physik, Philosophie und Musik und promovierte in Mathematik (Algebraische Geometrie). Sie war in der Industrie (Compiler-Compiler-Entwicklung) tätig und habilitierte in Informatik. Außerdem erlangte sie den venia legendi in Theoretischer Informatik.

Von 1981 bis 1991 fungierte sie als Professorin für Theoretische Informatik an der RWTH Aachen und forschte im Bereich der Theorie des Lernens (Induktive Inferenz), der Komplexitätstheorie und später in verschiedenen Feldern der „Künstlichen Intelligenz“, wie z.B. Wissensbasierte-, Konnektionistische und Natürlichsprachliche Systeme. Außerdem im Themengebiet Informatik und Gesellschaft. Weiter pflegte sie eine interdisziplinäre Zusammenarbeit mit der Soziologie, der Medizin und der Biologie (Schwerpunkt weiblicher Hirnatlas).

Seit 1991 ist sie Professorin für Informatik und Gesellschaft an der Universität Freiburg und hat ihren Sitz in der Fakultät für Mathematik und Physik und in der Fakultät für Angewandte Wissenschaften. 
Wächter, Christine, Ao. Univ. Prof. Dr., Professorin für Weiterbildung an der Abteilung Technik- und Wissenschaftsforschung, Universität Klagenfurt.

Christine Wächter ist stellvertretende Leiterin des IFZ und Ao. Universitäts-Professorin für Weiterbildung an der Abteilung Technik- und Wissenschaftsforschung der Fakultät für Interdisziplinäre Forschung und Fortbildung (IFF) der Alpen-Adria-Universität Klagenfurt (Dienstort Graz).

Sie absolvierte das Doktoratsstudium Anglistik/Amerikanistik, Nebenfach Kunstgeschichte an der Karl-Franzens-Universität Graz sowie das Aufbaustudium Technischer Umweltschutz an der Technischen Universität Graz. Sie ist Gründungsmitglied und war von 1991 bis 1999 Leiterin des IFZ und leitet jetzt den Projektbereich Frauen - Technik Umwelt. Sie ist ferner Mitglied des wissenschaftlichen Beirates der Interuniversitären Koordinationsstelle für Frauen- und Geschlechterforschung Graz, Vorstandsmitglied des Vereins Institut für Bildungsrecht und Bildungspolitik und Peer Evaluatorin für den österreichischen Fachhochschulrat. Weiterhin ist sie Gründungsmitglied der Projektgruppe FIT Frauen in die Technik.

Christine Wächter hat langjährige Lehrerfahrung an zahlreichen Universitäten. Arbeitsschwerpunkte: Technik-Bildung und Geschlecht, Feministische Perspektiven nachhaltiger Technikgestaltung, Konzepte zur quantitativen und qualitativen Verbesserung der Situation von Frauen in technischen Ausbildungs- und Berufswegen.

Weishoff-Houben, Michaela, Dr. med., Fachärztin für Hygiene und Umweltmedizin.

Michaela Weishoff-Houben studierte Humanmedizin an der Universität Köln und an der RWTH, Aachen und erhielt 1987 ihre Approbation als Ärztin. Seit 2003 belegt sie den Zusatzstudiengang Public Health an der Heinrich Heine-Universität Düsseldorf.

Ihre Dissertation an der Medizinischen Fakultät der RWTH Aachen schrieb sie 1990 über die Inzidenz, die Zusammensetzung und den Verlauf zirkulierender Immunkomplexe bei bioptisch gesicherten Glomerulonephritiden.

Seit 1988 ist Michaela Weishoff-Houben am Institut für Hygiene und Umweltmedizin der RWTH Aachen mit den Schwerpunkten: Public Health, Umweltepidemiologie, Krankenhaushygiene tätig und erhielt 2000 die Weiterbildungsermächtigung von der Ärztekammer Nordrhein für das Gebiet Hygiene und Umweltmedizin.

Sie ist seit 2002 verantwortlich für die Organisation und Umstrukturierung der Lehrer für die Fächer: Querschnittsbereiche Hygiene, Um- 
weltmedizin, Öffentliches Gesundheitswesen und Public Health im Modellstudiengang Humanmedizin der Medizinischen Fakultät der RWTH Aachen. Außerdem ist sie Dozentin an der Fakultät für Gesundheitswissenschaften, School of Public Health, Universität Bielefeld.

Wolf, Henrike, Dr., Projektkoordination des Mentoring-Programms TANDEMplusMED, RWTH Aachen.

Henrike Wolf ist promovierte Soziologin. Nach ihrer Ausbildung zur Physiotherapeutin und Gymnastiklehrerin studierte sie Soziologie, Pädagogik und Politische Wissenschaften an der RWTH Aachen. Von 1996-2005 war sie dort als Wissenschaftliche Mitarbeiterin in diversen empirischen, anwendungsorientierten Projekten in den Bereichen Frauenforschung, politische Soziologie sowie Bildungs- und Umweltsoziologie tätig. 2004 promovierte sie zum Thema „Partizipation und Lokale Agenda 21“.

Seit Ende 2005 ist sie Projektkoordinatorin des Mentoring-Programms TANDEMplusMED zur Förderung von Wissenschaftlerinnen in der Medizin. 


\section{Gender Studies}

Margarete Menz

Biographische

Wechselwirkungen

Genderkonstruktionen und »kulturelle Differenz" in den Lebensentwürfen binationaler Paare

Dezember 2007, ca. 320 Seiten, kart., ca. $31,80 €$,

ISBN: 978-3-89942-767-7

Rita Casale,

Barbara Rendtorff (Hg.)

Was kommt nach der

Genderforschung?

Zur Zukunft der feministischen

Theoriebildung

November 2007, ca. 280 Seiten,

kart., ca. $27,80 €$,

ISBN: 978-3-89942-748-6

Ute Frietsch,

Konstanze Hanitzsch, Jennifer John,

Beatrice Michaelis (Hg.)

Geschlecht als Tabu

Orte, Dynamiken und

Funktionen der De/Thema-

tisierung von Geschlecht

November 2007, ca. 260 Seiten, kart., zahlr. farb. Abb., ca. 25,80 €,

ISBN: 978-3-89942-713-4

Ulrike Brunotte,

Rainer Herrn (Hg.)

Männlichkeiten und Moderne

Geschlecht in den

Wissenskulturen um 1900

Oktober 2007, ca. 280 Seiten,

kart., ca. $28,80 €$,

ISBN: 978-3-89942-707-3
Katrin Oltmann
Remake - Premake

Hollywoods romantische

Komödien und ihre Gender-

Diskurse, 1930-1960

Oktober 2007, ca. 336 Seiten,

kart., ca. $29,80 €$,

ISBN: 978-3-89942-700-4

Corinna Tomberger

Das Gegendenkmal

Geschichtspolitik,

Erinnerungskultur und

Geschlecht in der neueren

Bundesrepublik

Oktober 2007, ca. 36o Seiten, kart., zahlr. Abb., ca. 33,80 €, ISBN: 978-3-89942-774-5

\section{Sylvia Pritsch}

\section{Rhetorik des Subjekts}

Zur textuellen Konstruktion des Subjekts in feministischen und anderen postmodernen

Diskursen

Oktober 2007, ca. 480 Seiten,

kart., ca. $38,80 €$,

ISBN: 978-3-89942-756-1

Anette Dietrich

"Weiße Weiblichkeiten"

Konstruktionen von »Rasse» und Geschlecht im deutschen Kolonialismus

Oktober 2007, ca. 36o Seiten,

kart., ca. $29,80 €$,

ISBN: 978-3-89942-807-o

Sven Glawion,

Elahe Haschemi Yekani,

Jana Husmann-Kastein (Hg.)

Erlöser

Figurationen männlicher

Hegemonie

September 2007, ca. 210 Seiten,

kart., ca. $24,80 €$,

ISBN: 978-3-89942-733-2

\section{Leseproben und weitere Informationen finden Sie unter:}

www.transcript-verlag.de 


\section{Gender Studies}

Nadja Sennewald

\section{Alien Gender}

Die Inszenierung von

Geschlecht in Science-

Fiction-Serien

August 2007, ca. 275 Seiten, kart., zahlr. Abb., ca. 27,80 €,

ISBN: 978-3-89942-805-6

\section{Gabriele Dietze}

\section{Weiße Frauen in Bewegung}

Genealogien und Konkurrenzen von Race- und Genderpolitiken

August 2007, ca. 450 Seiten,

kart., ca. $31,80 €$,

ISBN: 978-3-89942-517-8

\section{Claudia C. Ebner}

\section{Kleidung verändert}

Mode im Zeichen der

Cultural Studies

Juli 2007, ca. 240 Seiten,

kart., ca. $24,80 €$,

ISBN: 978-3-89942-618-2

\section{Carmen Leicht-Scholten (Hg.)}

"Gender and Science"

Perspektiven in den Natur- und

Ingenieurwissenschaften

Juli 2007, 188 Seiten,

kart., $21,80 €$,

ISBN: 978-3-89942-674-8

\section{Hedwig Wagner}

\section{Die Prostituierte im Film}

Zum Verhältnis von Gender und Medium

Juli 2007, 324 Seiten,

kart., $29,80 €$,

ISBN: $978-3-89942-563-5$

Tanja Maier

\section{Gender und Fernsehen}

Perspektiven einer kritischen

Medienwissenschaft

Juli 2007, ca. 300 Seiten,

kart., ca. $29,80 €$,

ISBN: 978-3-89942-689-2
Lutz Hieber, Paula-Irene Villa

Images von Gewicht

Soziale Bewegungen, Queer

Theory und Kunst in den USA

Mai 2007, 262 Seiten,

kart., $26,80 €$,

ISBN: 978-3-89942-504-8

Ingrid Hotz-Davies,

Schamma Schahadat (Hg.)

Ins Wort gesetzt,

ins Bild gesetzt

Gender in Wissenschaft, Kunst und Literatur

Mai 2007, 310 Seiten,

kart., 30,80 €,

ISBN: $978-3-89942-595-6$

Jürgen Martschukat,

Olaf Stieglitz (Hg.)

Väter, Soldaten, Liebhaber

Männer und Männlichkeiten in der Geschichte Nordamerikas.

Ein Reader

Februar 2007, 432 Seiten,

kart., $32,80 €$,

ISBN: 978-3-89942-664-9

Bettina Bock von Wülfingen

Genetisierung der Zeugung

Eine Diskurs- und Metaphernanalyse reproduktionsgenetischer Zukünfte

Februar 2007, 374 Seiten, kart., $30,80 €$,

ISBN: 978-3-89942-579-6

Roswitha Muttenthaler,

Regina Wonisch

Gesten des Zeigens

Zur Repräsentation von Gender und Race in Ausstellungen

Januar 2007, 268 Seiten,

kart., zahlr. Abb., 26,80 €,

ISBN: 978-3-89942-580-2

\section{Leseproben und weitere Informationen finden Sie unter:}

www.transcript-verlag.de 